Portland State University

PDXScholar

Spring 5-10-2019

\title{
A Scoring Model to Assess Organizations' Technology Transfer Capabilities: the Case of a Power Utility in the Northwest USA
}

João Ricardo Lavoie

Portland State University

Follow this and additional works at: https://pdxscholar.library.pdx.edu/open_access_etds

Part of the Technology and Innovation Commons

Let us know how access to this document benefits you.

\section{Recommended Citation}

Lavoie, João Ricardo, "A Scoring Model to Assess Organizations' Technology Transfer Capabilities: the Case of a Power Utility in the Northwest USA" (2019). Dissertations and Theses. Paper 4995.

https://doi.org/10.15760/etd.6871

This Dissertation is brought to you for free and open access. It has been accepted for inclusion in Dissertations and Theses by an authorized administrator of PDXScholar. Please contact us if we can make this document more accessible: pdxscholar@pdx.edu. 
A Scoring Model to Assess Organizations' Technology Transfer Capabilities: The Case of a

Power Utility in the Northwest USA

by

João Ricardo Lavoie

A dissertation submitted in partial fulfillment of the requirements for the degree of

Doctor of Philosophy

in

Technology Management
Dissertation Committee:
Tugrul Daim, Chair
Judith Estep
Elias Carayannis
Loren Lutzenhiser

Portland State University

2019 
(C) 2019 João Ricardo Lavoie 


\begin{abstract}
This research intends to advance knowledge in the technology management field, most importantly in the study of organizations that develop technologies inhouse and wish to enhance their technology transfer performance while maintaining adherence between R\&D activities and overall business strategies. The objective was to build a multi-criteria decision-making model capable of producing a technology transfer score, which can be used by practitioners in order to assess and later improve their organizations' technology transfer capabilities - ultimately aiming to improve technology development as a whole. The model was applied to a major power utility organization in the Pacific Northwest of the United States. The introduction brings initial and basic information on the topic, along with the problem statement - this chapter is aimed at situating the reader on the boundaries of the topic while highlighting its importance within the technology management field of study. The second chapter is the literature review. It brings general and specific information on technology transfer, as well as its complexities, gaps, relationship with other fields and the characteristics of this topic within the energy realm. It also tries to shed a light on how the alignment between R\&D and business strategy is perceived by the literature, discussing some of the methods used and its shortcomings. Additionally, the literature review brings an analysis that builds the argument in favor of a continuous technology transfer process, and tries to show how it would be helpful in
\end{abstract}


aligning R\&D and business strategy. The third chapter presents the methodological approach - hierarchical decision modeling (HDM) aided by action research - which constitutes a methodological novelty piloted and validated throughout the development of the study. The fourth chapter details the model development process step-by-step, and the fifth chapter details the model application process with the analysis of the aforementioned organization. Additionally, results are interpreted and analyzed, and insights for the specific case and for technology managers in general are discussed. Lastly, the contributions of the study towards the advancement of the body of knowledge are discussed, as well as the study limitations and future research opportunities. 


\section{DEDICATION}

I dedicate this work to my wife and to my family who have stood by and supported me incessantly throughout this life-changing journey. 


\section{ACKNOWLEDGMENTS}

Despite the fact that this dissertation brings about the fruits of years of my personal dedication and study, it could not have been possible (not in the slightest) without the help of many remarkable individuals. Although I am aware that I might be leaving some of those remarkable people out, I would nevertheless like to express my most sincere gratitude and appreciation for their support.

Perhaps the strongest and most decisive supporter, Dr. Tugrul Daim has brilliantly advised me through every step of the way and in every imaginable fashion, from helping me to choose the right coursework to encouraging me to accept a job offer while still enrolled in the PhD program. I have nothing but the deepest respect, admiration, and gratitude for his invaluable participation in this study.

Dr. Judith Estep has been a symbol of success and virtue for me from the very beginning. Her kind yet decisive way of advising has helped me "steer the ship" into the right direction countless times, and I am very proud to have built my research off of hers. Dr. Loren Lutzenhiser has always been extremely encouraging and optimistic about this study and my potential as a researcher, and has offered me the chance to look at my research from a different, less technical perspective, which has greatly improved the final product. Dr. Elias Carayannis, although having joined the PhD Committee at a later stage than the others, has influenced me tremendously with his solid experience and expertise in the field, and has given me priceless pieces of feedback and insights that I have proudly implemented in my research. 
I would also like to acknowledge the support and dedication of Dr. Leong Chan, who has helped me in the early stages of this research and through the comprehensive exam.

The help of my fellow ETM PhD colleagues has been paramount to my success. I was blessed with the opportunity to work side by side with extraordinary folks who-I have no doubt—will make significant and extremely positive impacts in the world, regardless of where their careers might take them. In particular, I would like to express my sincere gratitude to Rafaa Khalifa, Edwin Garces, Chih-Jen Yu, Momtaj Khanam, Byung Sung Yoon, Husam Barham, and Amir Shaygan - who have shared with me thousands of gallons of coffee and all the ups and downs of the PhD journey.

This research would not have been possible without the active participation, input, and feedback from dozens of subject matter experts, who took time from their busy schedules to understand the research and to help in the validation, quantification, and application of the research model.

Lastly, I would like to thank God, who guided me here and enabled me to be who I am, and my wife and family, who helped and supported me in so many ways that I cannot even begin to describe - you have my unconditional love and respect. 


\section{TABLE OF CONTENTS}

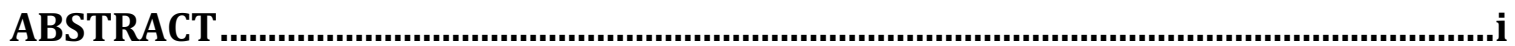

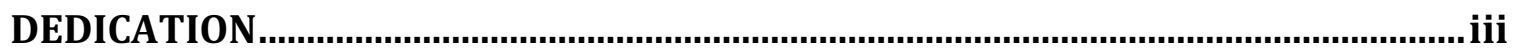

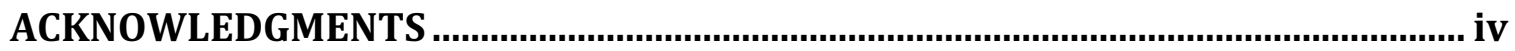

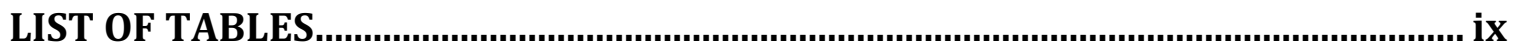

LIST OF FIGURES

LIST OF ABBREVIATIONS.......................................................................................ii

CHAPTER 1 - INTRODUCTION........................................................................... 1

PROBLEM STATEMENT ................................................................................................

CHAPTER 2 - LITERATURE REVIEW …….......................................................... 6

TECHNOLOGY TRANSFER (TT) …………………………………………………....6

Technology Transfer Definitions .................................................................................... 6

TT General Characteristics .............................................................................................. 9

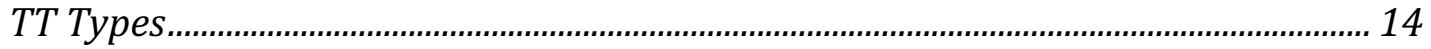

TT Mechanisms …..................................................................................................... 20

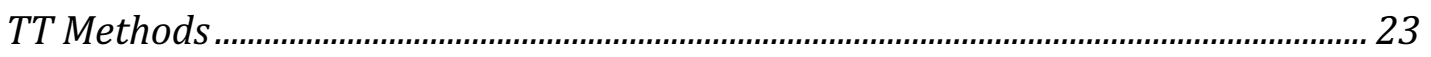

TT Application Areas ...................................................................................................... 25

TT Complexities, Challenges, and Gaps......................................................................... 26

TT Relationship with Other Fields and Concepts........................................................ 27

TECHNOLOGY TRANSFER/COMMERCIALIZATION IN THE ENERGY SECTOR....... 35

R\&D AND BUSINESS STRATEGY................................................................................... 46

General Aspects of R\&D Alignment with Business Strategy ......................................... 47

Methods and Tools for Aligning R\&D with Business Strategy..................................... 48

Gaps on the R\&D Alignment with Business Strategy.................................................... 50

STRATEGIC TECHNOLOGY CONTROL - A PROCESS.......................................................52

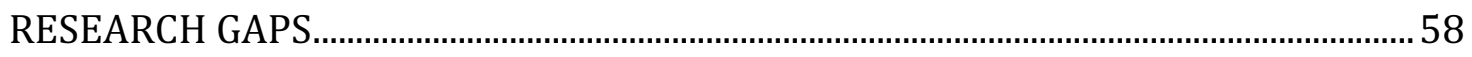

CHAPTER 3 - RESEARCH APPROACH AND METHODOLOGY …….......................61

RESEARCH GAPS, GOALS, AND QUESTIONS...................................................................61

RESEARCH METHODOLOGY........................................................................................62

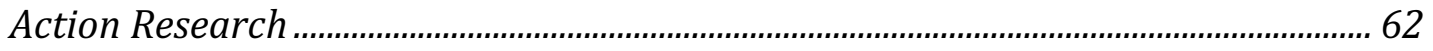

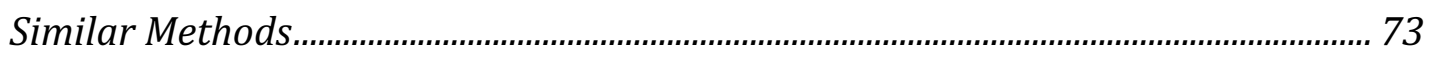


Why Action Research? …............................................................................................. 86

Hierarchical Decision Modeling (HDM) ........................................................................... 91

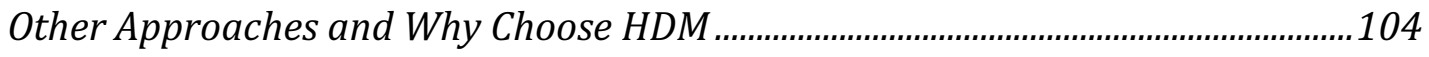

RESEARCH FRAMEWORK....................................................................................... 110

$1^{\text {st }}$ Step - AR and Literature Review ............................................................................111

$2^{\text {nd }}$ Step - Initial Model ...............................................................................................112

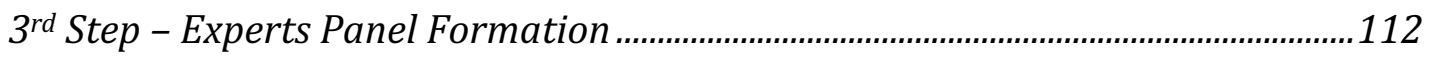

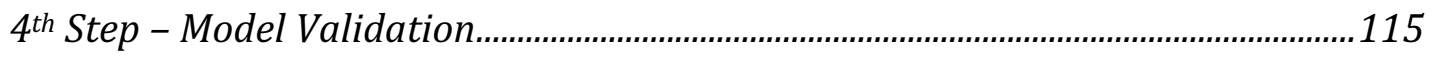

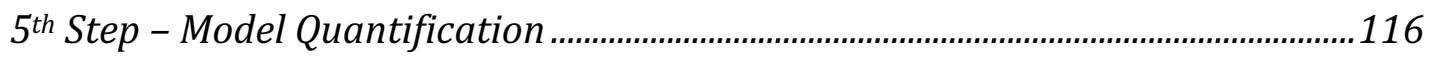

$6^{\text {th }}$ Step - Desirability Curves.....................................................................................117

$7^{\text {th }}$ step - Model Application and Analysis....................................................................117

RESEARCH VALIDITY ……..................................................................................... 129

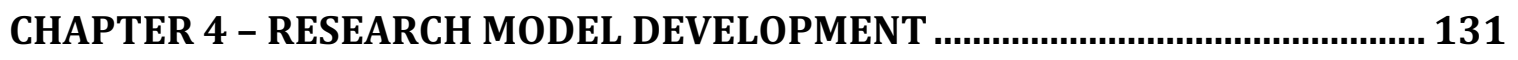

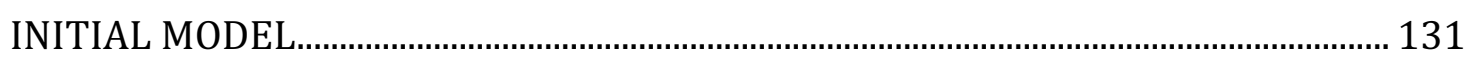

EXPERT PANELS FORMATION ……………………………..................................... 139

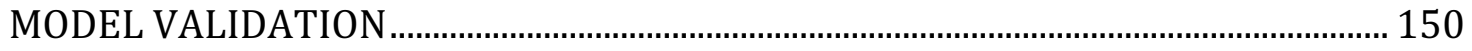

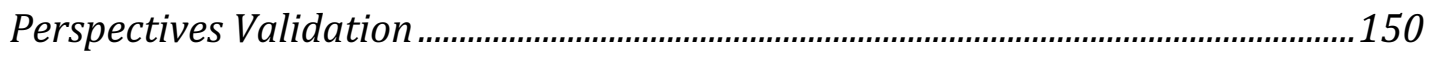

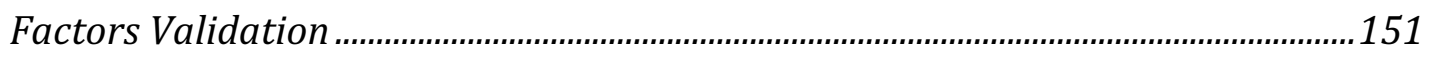

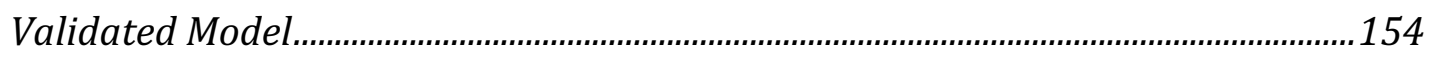

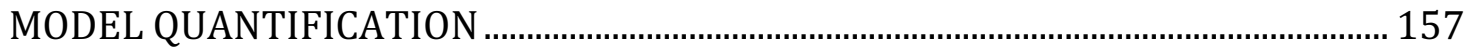

Perspectives Quantification ..........................................................................................157

Factors Quantification ..............................................................................................159

Summary of Model Quantification ................................................................................167

DESIRABILITY CURVES .......................................................................................... 170

CHAPTER 5 - RESEARCH MODEL APPLICATION ………........................................ 192

BPA'S TT PROCESS …………………....................................................................... 192

BPA's TT Process in Light of Literature.......................................................................193

THE ACTION RESEARCH PROJECT .............................................................................. 197

APPLICATION RESULTS ........................................................................................ 200

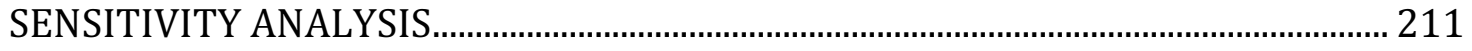

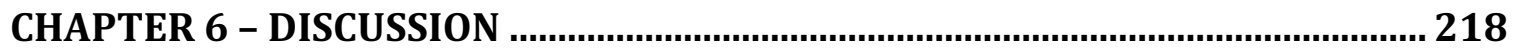




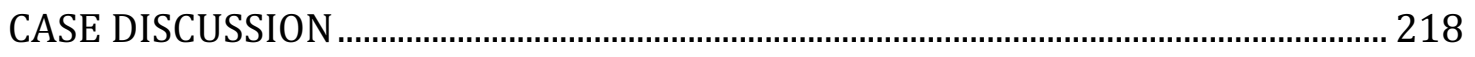

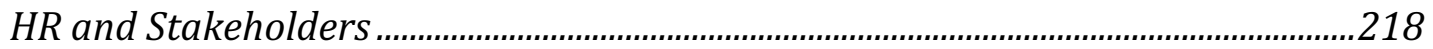

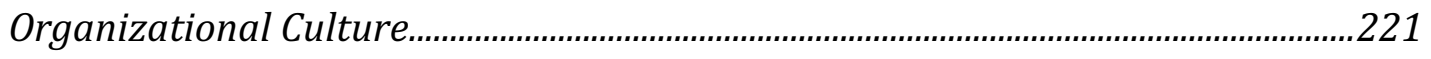

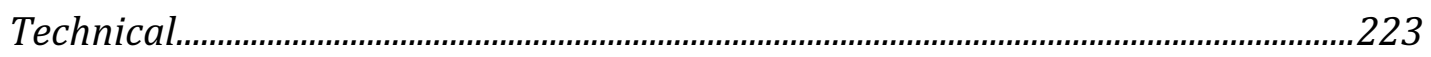

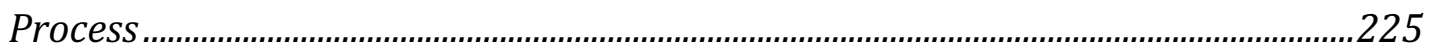

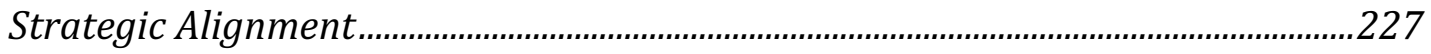

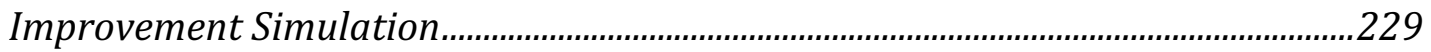

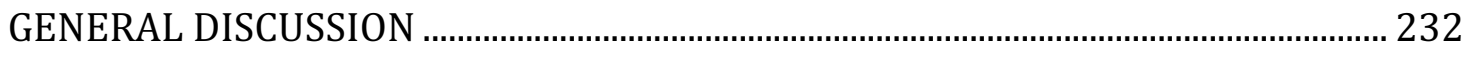

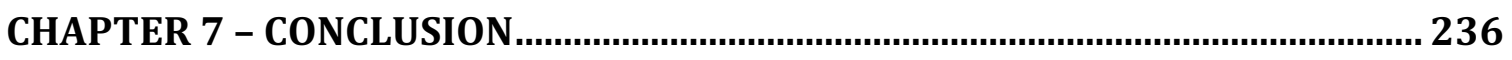

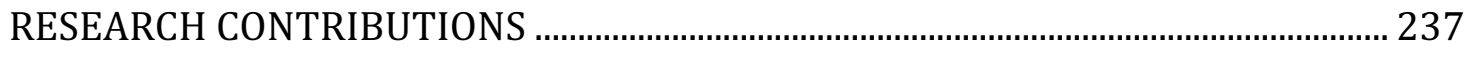

LIMITATIONS AND FUTURE RESEARCH OPPORTUNITIES......................................... 244

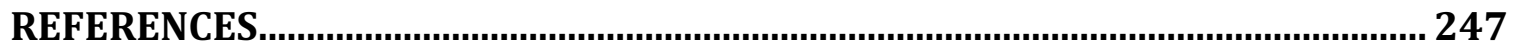

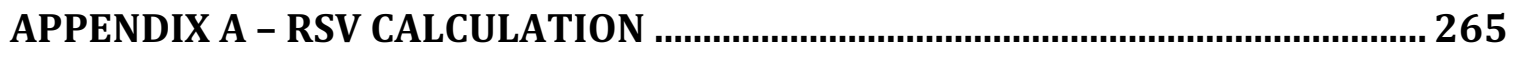

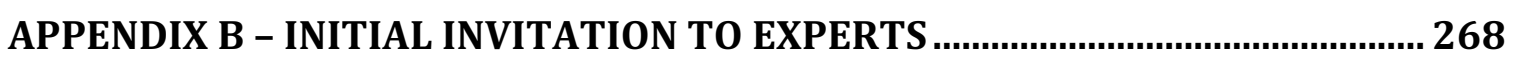

APPENDIX C - INVITATION FOR PERSPECTIVES VALIDATION .......................... 270

APPENDIX C - RESEARCH INSTRUMENT 1 (RI1) - PERSPECTIVES VALIDATION

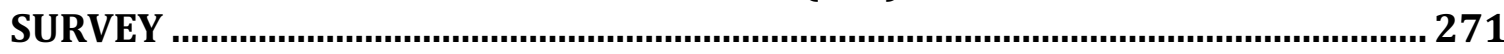

APPENDIX D - INVITATION FOR FACTORS VALIDATION ……............................. 273

APPENDIX E - RESEARCH INSTRUMENT 2 (RI2) - FACTORS VALIDATION

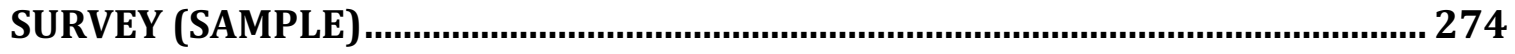

APPENDIX F - INVITATION FOR PERSPECTIVES QUANTIFICATION................... 277

APPENDIX G - RESEARCH INSTRUMENT 3 (RI3) - PERSPECTIVES

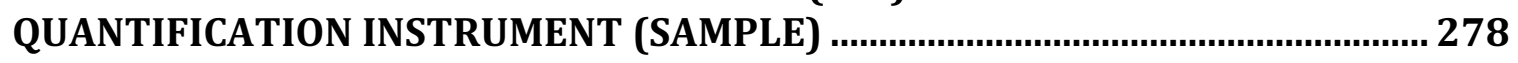

APPENDIX H - INVITATION FOR FACTORS QUANTIFICATION ........................... 283

APPENDIX I - RESEARCH INSTRUMENT 4 (RI4) - FACTORS QUANTIFICATION

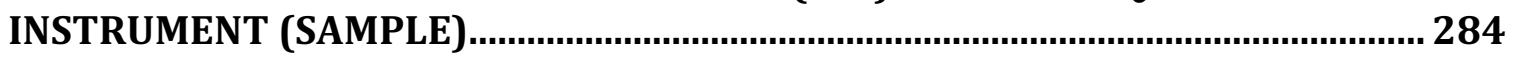




\section{LIST OF TABLES}

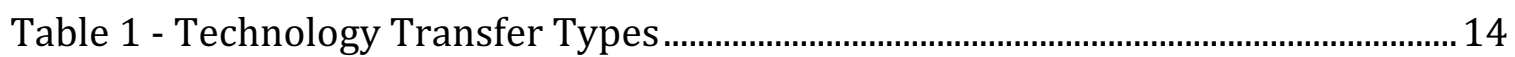

Table 2 - TT Mechanisms ................................................................................................22

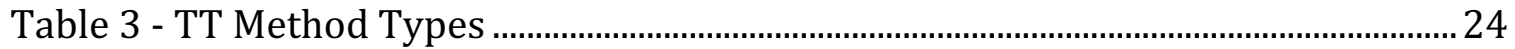

Table 4 - TT Methods and Tools ......................................................................................... 24

Table 5 - Technology Transfer Application Areas ...........................................................2. 25

Table 6 - Relationship between Technology Transfer and Commercialization............28

Table 7 - Relationship between Technology Transfer and Policy-Making.....................29

Table 8 - Relationship Between Technology Transfer and Technology Assessment 30

Table 9 - Relationship Between Technology Transfer and Technology Development

Table 10 - Relationship Between Technology Transfer and Product Development.. 32

Table 11 - Relationship Between Technology Transfer and Technology Forecasting

Table 12 - Relationship Between Technology Transfer and Technology Maturity....33

Table 13 - Relationship Between Technology Transfer and Technology Maturity....35

Table 14 - Technology Commercialization in the Energy Sector - Application Areas

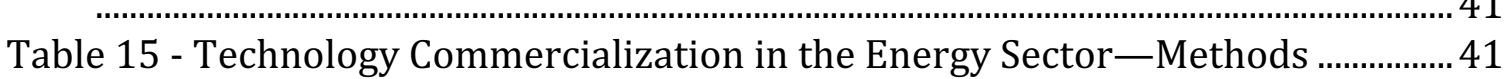

Table 16 - Technology Commercialization in the Energy Sector - Criteria..................... 43

Table 17 - R\&D and Business Strategy Alignment - Methods and Tools......................... 48

Table 18 - Action Research Application Areas ....................................................................67

Table 19 - Action Research Producing Changes ...............................................................69

Table 20 - Action Research and Auxiliary Methods ..........................................................

Table 21 - Case Study Objectives......................................................................................... 74

Table 22 - Interview Objectives ………………………………………………………......77

Table 23 - Participant Observation Objectives..................................................................... 81

Table 24 - Focus Groups Objectives .................................................................................... 85

Table 25 - Technology Transfer Method Types ................................................................ 105

Table 26 - Technology Transfer Methods and Tools ....................................................... 105

Table 27 - HDM Factors from Literature....................................................................... 131

Table 28 - HDM factors from Action Research ............................................................... 132

Table 29 - Description of Model Perspectives ................................................................... 133

Table 30 - Description of Model Factors ............................................................................ 134

Table 31 - List of Experts ................................................................................................ 142

Table 32 - Panel 1 ……………………………………………………………….... 144

Table 33 - Panel 2 ………………………………………………………………………... 144

Table 34 - Panel 3 …………………………………………............................................ 144

Table 35 - Panel 4............................................................................................................. 145

Table 36 - Panel 5 ........................................................................................................... 146

Table 37 - Panel 6 6................................................................................................................ 146

Table 38 - Panel 7 7.......................................................................................................... 146

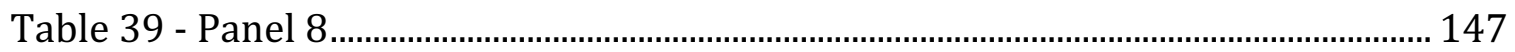




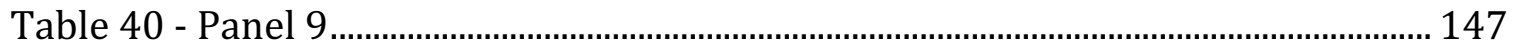

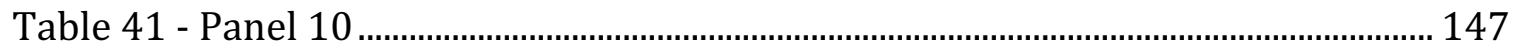

Table 42 - Panel 11 .......................................................................................................... 148

Table 43 - Panel 12 ................................................................................................................... 148

Table 44 - Perspectives Validation Results ........................................................................ 150

Table 45 - Factors Validation Results for "HR and Stakeholders" ................................ 151

Table 46 - Factors Validation Results for "Organizational Culture".............................. 151

Table 47 - Factors Validation Results for "Technical”...................................................... 152

Table 48 - Factors Validation Results for "Process" ............................................................. 152

Table 49 - Factors Validation Results for "Strategic Alignment" .................................... 152

Table 50 - Perspectives Quantification Results.................................................................. 158

Table 51 - HR and Stakeholders Factors Quantification Results................................. 159

Table 52 - Organizational Culture Factors Quantification Results ............................... 161

Table 53 - Technical Factors Quantification Results ..................................................... 163

Table 54 - Process Factors Quantification Results ......................................................... 164

Table 55 - Strategic Alignment Factors Quantification Results .................................... 166

Table 56 - Model Quantification Results Summary Table ............................................. 168

Table 57 - Summary of DC Values Assignment to BPA …................................................ 208

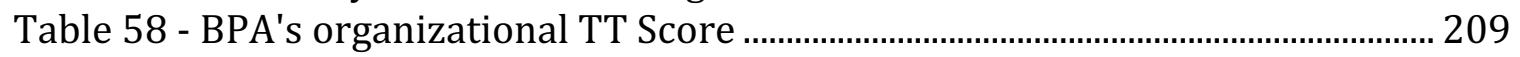

Table 59 - Sensitivity Analysis Scenario 1 Results .......................................................... 212

Table 60 - Sensitivity Analysis Scenario 2 Results ............................................................ 213

Table 61 - Sensitivity Analysis Scenario 3 Results ......................................................... 214

Table 62 - Sensitivity Analysis Scenario 4 Results ........................................................... 215

Table 63 - Sensitivity Analysis Scenario 5 Results .......................................................... 216

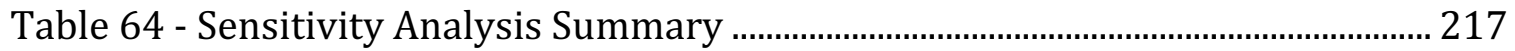

Table 65 - Simulation of Top Five Factors Improvement ............................................. 230 


\section{LIST OF FIGURES}

Figure 1 - TT Success Attributes Perspectives, adapted from [6] ....................................11

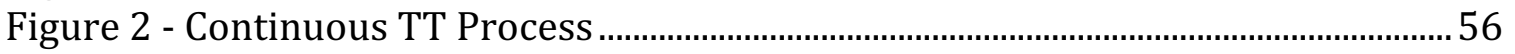

Figure 3 - TT as a Strategic Technology Control Process...................................................57

Figure 4 - Research Gaps, Goal and Questions ................................................................61

Figure 5 - Methodological Approach - AR and HDM Integration...................................... 91

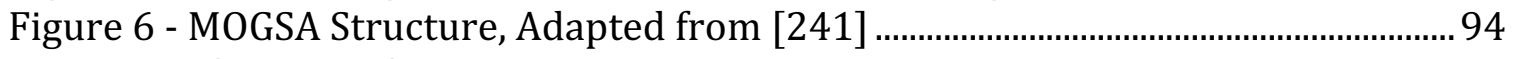

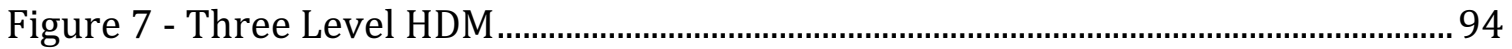

Figure 8 - Desirability Curve - Example 1 ……………………………………………...98

Figure 9 - Desirability Curve - Example 2 …………………………………………....... 99

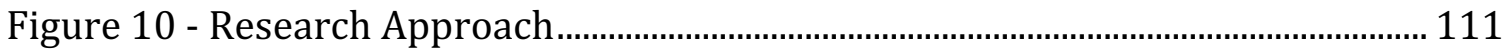

Figure 11 - Model Validation Framework …………………………………………….... 115

Figure 12 - Model Quantification Framework ………………………………………...... 116

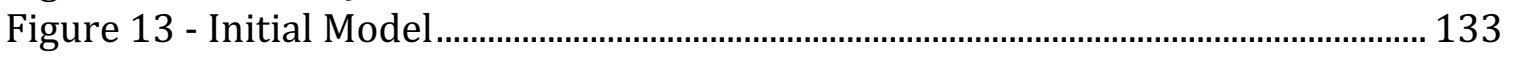

Figure 14 - Expert Panels Distribution ............................................................................ 141

Figure 15 - Validated HDM Model.................................................................................. 155

Figure 16 - Pre-validation Model vs. Post-Validation Model.......................................... 156

Figure 17 - Perspectives Quantification Chart .............................................................. 158

Figure 18 - HR and Stakeholders Factors Quantification Chart..................................... 160

Figure 19 - Organizational Culture Factors Quantification Chart .................................. 161

Figure 20 - Technical Factors Quantification Chart ........................................................ 163

Figure 21 - Process Factors Quantification Chart ............................................................. 165

Figure 22 - Strategic Alignment Factors Quantification Chart....................................... 166

Figure 23 - Model Quantification Results Summary Figure .......................................... 169

Figure 24 - TT team and Training Curve.......................................................................... 171

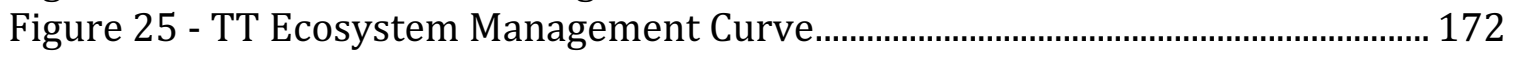

Figure 26 - Stakeholders Management Curve................................................................... 173

Figure 27 - Senior Management Involvement Curve..................................................... 174

Figure 28 - Communication and Knowledge Management Curve ................................. 175

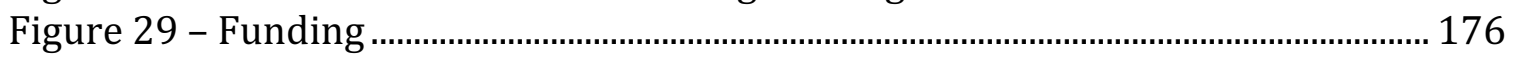

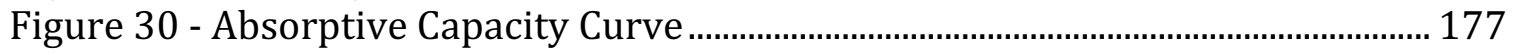

Figure 31 - Long Range Planning Curve ........................................................................ 178

Figure 32 - Innovative Culture Curve .............................................................................. 179

Figure 33 - TRL Assessment Curve.................................................................................. 180

Figure 34 - Technology Valuation Curve.......................................................................... 181

Figure 35 - Proposal Assessment Curve........................................................................ 182

Figure 36 - Benefit Management Curve................................................................................ 183

Figure 37 - Risk Management Curve................................................................................. 184

Figure 38 - Outcomes and Decisions Curve ……………………………………………... 185

Figure 39 - TT Mechanisms Management Curve .............................................................. 186

Figure 40 - Value, Impact and Applicability Management Curve................................... 187

Figure 41 - Business Plan and Use Case Curve............................................................... 188

Figure 42 - TT Planning, Control and Flexibility Curve .................................................... 189 
Figure 43 - Continuity of TT Process Curve........................................................................ 190

Figure 44 - Parallel Processes Integration Curve................................................................ 191

Figure 45 - Top five and Bottom Five Factors ................................................................. 240

Figure 46 - Most and Least Important Factors per Perspective ..................................... 241 


\section{LIST OF ABBREVIATIONS}

\begin{tabular}{|c|c|}
\hline AHP & Analytic Hierarchy Process \\
\hline ANOVA & Analysis of Variance \\
\hline AR & Action Research \\
\hline BIM & Building Information Modeling \\
\hline BIT & Business Integration Team \\
\hline BPA & Bonneville Power Administration \\
\hline CRADA & $\begin{array}{l}\text { Cooperative Research and Development } \\
\text { Agreements }\end{array}$ \\
\hline DOE & Department of Energy \\
\hline DR & Demand Response \\
\hline $\mathrm{EE}$ & Energy Efficiency \\
\hline ESTO Model & $\begin{array}{l}\text { Ecosystem, Strategy, Technology Platform, and } \\
\text { Organizing }\end{array}$ \\
\hline FDI & Foreign Direct Investment \\
\hline GUISP & $\begin{array}{l}\text { Government-University-Industry Strategic } \\
\text { Partnerships }\end{array}$ \\
\hline HAC & Hierarchical Agglomerative Clustering \\
\hline HDM & Hierarchical Decision Modeling \\
\hline HR & Human Resources \\
\hline ICC & Intra-Class Correlation Coefficient \\
\hline IP & Intellectual Property \\
\hline IRL & Integration Readiness Levels \\
\hline IT & Information Technology \\
\hline MCDM & Multi-criteria Decision-making \\
\hline MOGSA & Mission, Objectives, Goals, Strategies, Actions \\
\hline NEEA & Northwest Energy Efficiency Alliance \\
\hline NPD & New Product Development \\
\hline PLC & Product Life Cycle \\
\hline PMU & Phasor Measurement Units \\
\hline POC & Proof-of-Concept Center \\
\hline PV & Photo-Voltaic \\
\hline QFD & Quality Function Deployment \\
\hline R\&D & Research and Development \\
\hline RD3 & Research and Development Degree of Difficulty \\
\hline ROI & Return Over Investment \\
\hline RSV & Root Sum of Variances \\
\hline SNA & Social Network Analysis \\
\hline SRL & System Readiness Levels \\
\hline
\end{tabular}


TI

TOPSIS

TQM

TRL

TRM

TT

TTO

UK

VOT

WAPA
Technology Innovation

Technique for Order of Preference by Similarity to Ideal Solution

Total Quality Management

Technology Readiness Levels

Technology Roadmapping

Technology Transfer

Technology Transfer Office

United Kingdom

Voice of Technology

Western Area Power Administration 


\section{CHAPTER 1 - INTRODUCTION}

With a global economy that has dynamics as complex as they are today, and with customer expectations as high as they are, organizations regard innovation as a critical component of their businesses, regardless of the sector they compete in. Innovation, in turn, can be translated into more and better processes and products to minimize costs and to fulfill the ever-increasing and ever-more complex requirements and expectations of consumers. Having this scenario as an inescapable reality, research and development (R\&D) activities have become more and more crucial since the innovation that organizations need, to a large extent, comes from these activities. Once seen as purely creative and non-manageable process, $R \& D$ has started to attract the attention of managers who see it as an opportunity to enhance innovation, and the whole performance of an organization, by means of managing research and development efforts. In this context, many managerial processes have been created and improved, e.g., project management, program and portfolio management, new product development, and roadmapping, among others.

Technology transfer (TT) is one of those processes, and although it has been subject of research for at least 45 years, it is still a very unclear process and presents several research opportunities. Technology transfer is a multi-faceted process [1]. It is a very complex problem that involves multiple perspectives and disciplines [2][3][4]. Notwithstanding being less mentioned than other managerial processes when it comes to enhancing R\&D performance and overall organizational performance, technology transfer plays a critical role - especially for high technology 
organizations. According to several authors, an organization with good technology transfer capabilities has a competitive advantage over rivals, bringing better products and solutions to the market, faster and more easily [5][6], [7]. Having been once regarded as a one-time event to be carried out after a technology is completely developed, technology transfer has evolved, and is considered as much more than simply deploying or transferring technologies after they are fully developed. It is a crucial process that runs in parallel with technology development and can truly affect its outcomes. In 1976, Robbins and Milliken were already regarding TT as part of the innovation process. Nowadays, there seems to be a consensus among scholars that this process should be properly managed if it is to result in benefits to the organization [8][9].

The amount, intensity, and sources of knowledge and technical expertise for research and development can vary significantly from organization to organization. High technology and innovative organizations, however, tend to intensely develop technologies internally [10]-[12]. Nevertheless, it is not easy to strategically align technological research with business applications [1], [13]. The result of a misalignment between business strategy and technology development would be failed products and financial loss to the organization. In a 2013 article written for the website Techworld, Sophie Curtis argues that four in every five technologies fail before hitting the market [14]. According to Baruah and Ward [15], intrapreneurship projects, which are entrepreneurial dynamics within existing organizations to develop technologies and products, fail $70 \%$ to $90 \%$ of the time [16]. This misalignment often times augments the chasm observed between technology 
development and product development, or between product development and product launch, which is sometimes referred to as "the valley of death". Especially for technology-push innovations, there is a gap between technology development and product development [17], [18]. However problematic it is to fill this gap (or bridge this chasm), technology transfer efforts have the potential to mitigate this problem. According to Estep (2017), the greater the TT efforts, the easier it is to overcome the "valley of death" in research projects [19].

In general terms, the objective of the proposed research is to develop a technology transfer score, aiming to help organizations in measuring and enhancing their internal technology transfer capabilities, ultimately leading to better R\&D performance. The methodology proposed is Action Research followed by Hierarchical Decision Modeling (HDM). This Multi-Criteria Decision-Making method makes it possible to identify crucial criteria and factors influencing an organization's TT capabilities, while measuring its performance and providing valuable insights on weaknesses and strengths. The specific goals and research questions are listed and explained in more detail in later sections.

The remainder of this dissertation is as follows: A succinct problem statement; a literature review touching upon technology transfer and related fields, including an explanation and analysis of technology transfer as a technology control process; a research gap analysis, with research objectives and questions; research approach, with methodology, data collection, data analysis, and research framework; the research model development, with expert panels formation, model validation and model quantification; the research model application and its results; discussion of 
results in terms of the specific case and in terms of general expectations and model usefulness for technology managers; a conclusion section with a brief discussion on research contributions, limitations, and future research opportunities.

\section{Problem Statement}

The importance of technology transfer is easily identified in the literature, but more than only the transfer itself, the process and how it is managed are also very important. To have a proper TT process in place is vital. Magnussen and Johansson (2008) explain that, for any transfer to be successful, not only do organizations need to be aware of what is going to be transferred and when, but also how the process is being conducted [9]. Although there is a vast literature on technology transfer, hitherto the majority of studies investigate external technology transfer situations, leaving internal technology transfer somewhat unattended. As factors and characteristics might be different when dealing with internal TT versus dealing with external TT, organizations might be looking at a too broad of an array of models, criteria, and solutions. This might lead to confusion that, in turn, becomes more of a hindrance than a help.

Moreover, technology transfer is not entirely well understood and standardized in organizations - especially internal TT. When it comes to identifying important factors for an effective TT process, and measuring methods in order to provide organizations with TT situational awareness and suggestions for improvements, there is no study or model comprehensive enough. There is a lack of a quantitative assessment framework to measure and guide practitioners and 
organizations in enhancing their technology transfer processes, skills, and practices. The result of this situation, in part, is a difficulty in synchronizing research and development with business applications and corporate strategy, and also an increase in failed technologies, either before being commercialized or shortly after being brought to the market.

To summarize the problem:

- Organizations need better technology transfer capabilities to increase the alignment between corporate strategy and R\&D, and to enhance their internal R\&D and technology development performance.

- There is not, as of now, an appropriate and comprehensive enough model or method to measure an organization's internal technology transfer capabilities. As it will be further discussed in this dissertation, the objective of this study is to develop a score to measure organizations' internal technology transfer capabilities. In order to achieve this objective, a hierarchical decision model was built and applied at the Bonneville Power Administration, an electric utility in the Pacific Northwest region of the United States. 


\section{CHAPTER 2 - LITERATURE REVIEW}

This chapter brings the theoretical foundations and literature analysis that laid the groundwork for the achievement of this study's objectives.

\section{Technology Transfer (TT)}

In this first part a broad picture of technology transfer is presented according to the eyes of scholars-what it is, its major characteristics, methods and applications areas, relationship with related fields, and main themes discussed in the literature.

\section{Technology Transfer Definitions}

Technology transfer is a multidisciplinary effort, involving multiple perspectives that have to be taken into account simultaneously in order for the transfer to be successful. Technology commercialization planning, for instance, is not only composed of scientific and technological development, but also encompasses other functions of an organization, e.g., customer relationship management, marketing, financial management, and market management [20]. The very definition of technology transfer can be confusing and emanate different interpretations. Several different definitions are observed across the literature, each one with slightly different perspectives and nuances. Although these definitions have common points and do not seem to be diametrically opposed to each other, this plurality of definitions can be troublesome and harm practitioners in their pursuit of a more effective, smoother, faster, and more efficient technology transfer. As academia is always at the forefront of knowledge creation, scholars still need to come to a consensus on what 
is the best definition for technology transfer, or what are the best definitions, depending on the type of transfer, type of organization, purpose, and other factors.

For Zhao and Reisman, the definition would change according to the discipline or knowledge field [21]:

- Economy: the focus would be on technology production and design.

- Sociology: the focus would be on social aspects.

- Anthropology: the focus would be on cultural change.

In the early days of TT research, Bar-Zakay stated that technology transfer happens when a technology generated in one context is used in another one [2]. More recently, it was defined as bringing technical expertise from one organizational reality to another [3]. Heinzl et al. bring the concept of commercialization onto the scene when they state that TT is the "process of developing practical applications for the results of scientific research", and the "process of moving technology from an institution of science base to an industrial organization, which successfully commercializes the technology" [22]. Also focusing on commercialization aspects but including a technology diffusion element, Meseri and Maitai state that "technology transfer is a complex process, involving the diffusion of basic research and its ultimate commercialization" [23]. Following the same line, Rogers et al. argue that it is the movement of a technology from a research organization to a receiver. The process is complete when the transferred technology is commercialized and sold in the market as a product [24]. Focusing more on the geographical aspect, Liu defines it as transferring technologies from one organization or location to another [25]. Bringing 
the terminology of mechanisms, Amesse and Cohendet argue that TT happens when people or organizations, using different mechanisms, come together and interact to interchange technologies [26].

Carayannis [27], while discussing previous relevant literature [28], [29], defines technology as a knowledge system, and asserts that this definition would provide the basis for regarding technology transfer also in terms of information exchange and cognitive/linguistic abilities. With that in mind, the author moves on to link technology transfer with the concept of technology learning, which would be the cognitive ability that functions as a tool through which technology transfer is conducted and managed [27].

Another building block of technology transfer is the ability to acknowledge, understand, and workaround various transfer barriers, e.g., technical, social, cultural, economic - the so-called innovation diplomacy [30]. The concept of technology transfer, thus, can be translated into a knowledge transfer process where explicit and tacit knowledge are put into use after being absorbed by different groups of people [31] through a communication avenue [32]. Restated, information is communicated from a first group of people (or organization) to a second group of people (or organization), the latter using it to its own advantage [27], [30], e.g., research results are communicated and turned into products to be commercialized [33].

In summary, technology transfer will always involve the movement of knowledge to be used, further developed, or commercialized by another set of people, be it within the same firm, across different organizations, or even different countries. 
The specifics of the process will vary significantly depending on the type and purpose of transfer.

\section{TT General Characteristics}

Technology transfer is a complex process. It requires an interdisciplinary approach [2], [34], and it is a multi-faceted process, not a simple one [1]. Instead of having just a contemplative nature and serving only as an auxiliary process for other managerial process (e.g., project management), it has to give information to, and be the basis upon which managers will make decisions and take actions. The TT process involves "go / no-go" decision points [2].

The requirements for an organization in order to conduct a transfer, according to Seaton and Cordey-Hayes [35] are:

- Technical functions should support the business priorities the organization has set, and create new opportunities based on these priorities.

- All functions should be integrated in order for the organization to work as a network (with internal and external connections).

- Employees and managers should be educated on how the organization and the process works.

Bar-Zakay has dealt with the questions of the skills required for technology transfer. The author argues that, for both sides (donor and receiver), these skills are essential: System analysis, technological forecasting, long-range planning, and project-related intelligence [2]. 
The question of success factors has also been dealt with in the literature. In order to be successful, TT requires the development of technology markets and the development of technology valuation methods [36]. Leonard-Barton and Sinha highlighted two important factors for a successful technology transfer. The authors discuss how organizations have to undertake a sort of adaptation process (either for the new technology to be adapted to the organizational environment, or the other way around), and how the communication and interaction between developers and users should be intense from day one [37]. Franza and Grant have listed success attributes by player types, namely developer, acquirer, and both, showing the traits and characteristics each group ought to have in order to thrive.

In a comprehensive research work, Estep identified four categories of technology transfer success attributes perspectives and factors: Research domain, technology recipient domain, technology characteristics, and interface strategy, as shown in Figure 1 [6]. 


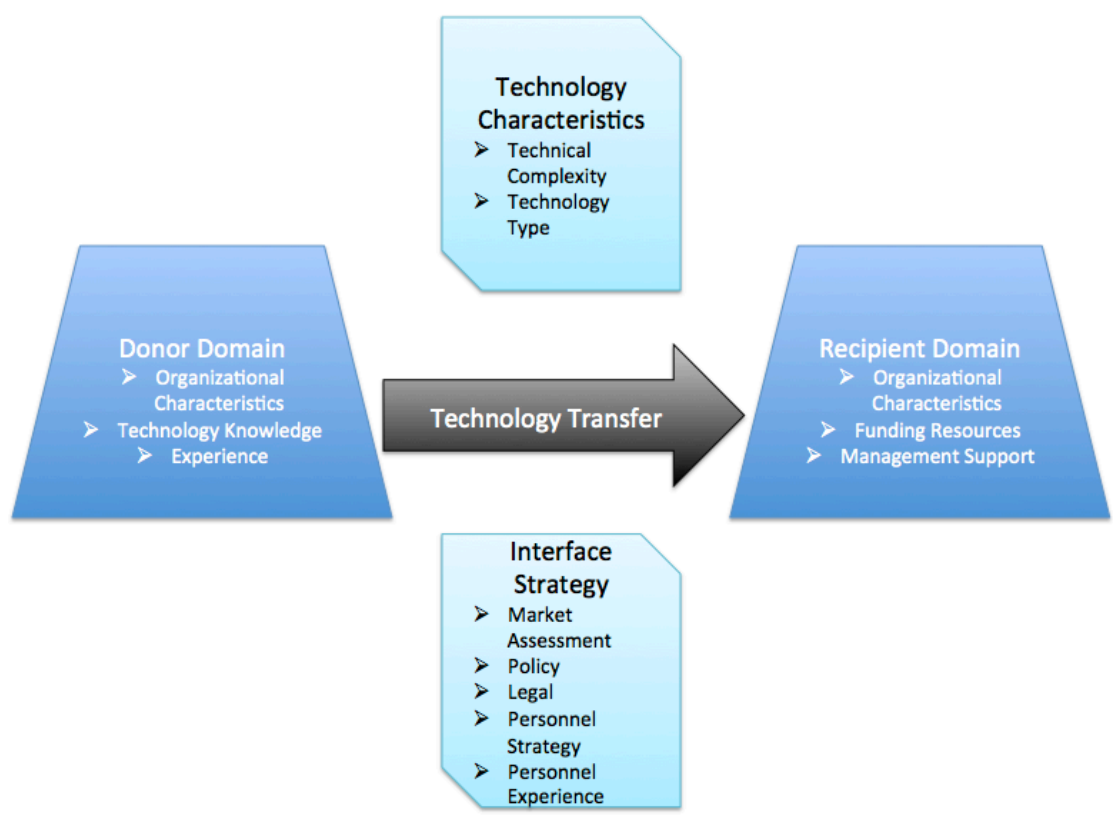

Figure 1 - TT Success Attributes Perspectives, adapted from [6]

Previous studies have also tried to identify and define the stages contained in a technology transfer process. According to the framework developed by Bar-Zakay in 1971, technology transfer would contain four stages: Search-when one searches for technologies to be transferred; adaptation-when one adapts the technology and the organization for them to work together; implementation-when one does the actual transfer and deploys it in the new environment; and maintenance-when one makes sure the technology is and will work properly in the long-run [2]. In a more simplistic fashion, Seaton and Cordey-Hayes have defined the TT stages as idea scanning, communication, assimilation within the organization, and idea application with a purpose, which would be higher business effectiveness or competitive advantage [35]. 
Some studies were conducted with the aim of delineating the TT process and the elements or factors involved in the process. For Heinzl et al., the important factors for technology transfer are: Transfer object, transfer mechanism, intellectual property (IP) rights, absorptive capacity, and support structures [22]. Transfer object is the 'what', it is the item or element to be transferred from the donor to the receiver. The mechanism is the 'how', the way or the vehicle through which the transfer is conducted. IP rights comprise all legal considerations over patents, brand, trademarks, and other intellectual property aspects. Absorptive capacity refers to the skills of an entity of receiving, understanding and properly using new information and knowledge [38]. The support structures are organizational arrangements and entities that provide aid and assist the transfer process. The Technology Transfer Office (TTO) in universities would be a good example [22]. Nobelius lists three elements of the TT process: Strategic and operational synchronization, transfer scope, and transfer management [1]. The first part refers to the alignment between research and technology development efforts and the overall business strategy and application. The second part refers to the transfer object or the well-defined 'what'. The third and last part refers to the 'how', i.e., the mechanism and the technology transfer process itself and how to manage it. Bozeman also dealt with specific dimensions of effectiveness for TT. The author lists as important dimensions: Transfer agent, transfer medium, transfer object, transfer recipient, and demand environment [3].

The literature also provides more specific criteria that should be assessed during a transfer, or questions that should be posed in order to measure the 
transferability of an item, or to measure the potential for transfer. Both qualitative and quantitative criteria are mentioned as vital to assess technology transfer aspects. In his 1977 article, Bar-Zakay lists two sets of criteria / questions to be checked on the donor and the recipient side. The questions relate to the number of people involved, training, interaction, planning aspects, and complexity of transfer, among others [34]. Bozeman lists as important criteria: Out-the-door, market impact, economic development, political reward, opportunity costs, and scientific and technical human capital [3]. Out-the-door would relate to the question of whether a technology has actually been received by another party. Market impact relates to the question of profitability and market-share changes caused by the transfer. Economic development relates to market impact questions on a regional or country level. Political reward relates to political gains derived from the transfer, such as more and better access to funding. Opportunity costs relates to other uses for resources or other agents and items. Scientific and technical human capital relates to the impact and advancements to technical skills and infrastructure caused by the transfer. Heinzl et al. provides three categories, each of which with associated factors and dimensions to be taken into account: providing agent, receiving agent, and environment [22]. Baek et al. also list specific criteria. On the qualitative side, the authors list the analysis of technology degree of contribution. On the quantitative side, the authors list the analysis of expected returns, cost structure estimation, and market estimation [36].

Technology transfer, although it can be strictly confined to the boundaries of one organization (when it is an internal TT), usually involves two or more entities. Moreover, even if it is an internal process, it will certainly involve several different 
groups and departments. The collection of these entities is commonly referred to as the technology transfer ecosystem. A TT ecosystem is a collection of stakeholders and entities (a system) that work together to promote a better transfer and to create value. Meseri and Maital argue that a systems approach is essential for technology transfer [23]. Some of the entities mentioned are: Science parks, research centers, incubators, TTO's, innovation and commercialization networks; and proof of concept centers (POC) [22]. Technology transfer offices (TTO) are also mentioned in other studies [39], [40].

\section{TT Types}

Several types of technology transfer are mentioned and studied in the literature, as Table 1 shows.

Table 1 - Technology Transfer Types

\begin{tabular}{|c|c|}
\hline \multicolumn{2}{|c|}{ Technology Transfer Types } \\
\hline Type & References \\
\hline Internal & {$[25],[37],[38],[41]-[44]$} \\
\hline External & {$[2],[25],[34],[36],[41]$} \\
\hline Domestic & {$[3],[45]-[47]$} \\
\hline International & {$[2],[41],[48]-[55]$} \\
\hline Military to Civilian & {$[34],[56]-[58]$} \\
\hline National Labs to Private Sector & {$[3],[24],[59]-[61]$} \\
\hline Universities to Private Sector & {$[3],[22]-[24],[62],[63]$} \\
\hline Research to Development & {$[60],[64]-[67]$} \\
\hline Precompetitive / Competitive/ Coopetitive & {$[55],[68]-[72]$} \\
\hline
\end{tabular}




\begin{tabular}{|c|c|}
\hline $\begin{array}{c}\text { Consortium to Consortium / Consortium to } \\
\text { Firm / Firm to Firm }\end{array}$ & [55], [73]-[78] \\
\hline Vertical / Horizontal & {$[55],[79]-[84]$} \\
\hline
\end{tabular}

The succinct definitions of each type are as follows:

- Internal-intra-firm transfer; the process occurs within the same organization.

- External-the transfer occurs between different organizations, regardless of their locations.

- Domestic-the transfer occurs within the same country.

- International-the transfer occurs between different countries.

- Military to Civilian - military technologies to be transferred to civilian usage / private companies.

- National Labs to Private Sector-technologies developed within federal labs to be commercialized by private companies.

- Universities to Private Sector-technologies developed within universities to be commercialized by private companies.

- Research to Development-the transfer from the technology development process into the product development process. It also involves the transfer of technologies in the early stages of development.

- Precompetitive-collaborative transfer of technologies that build the basis of a particular sector and that benefit the sector as a whole, e.g., development of distribution channels. 
- Competitive-collaborative transfer of technologies that play a major role in a particular sector in terms of competitive advantage.

- Coopetitive-collaborative transfer of technologies that combines both competitive and precompetitive aspects, resulting in a win-win situation where all participants (although competitors) benefit from sharing key capabilities/knowledge.

- Consortium to Consortium-groups of organizations (consortia) transferring technologies to groups of organizations (consortia).

- Consortium to Firm-groups of organizations (consortia) transferring technologies to individual organizations (firm).

- Firm to Firm-individual organizations (firm) transferring technologies to individual organizations (firm).

- R\&D to Fuzzy Front-End - technologies being transferred from the technology development cycle into the new product development cycle.

- Horizontal—any technology transfer involving competitors.

- Vertical-technology transfer involving players placed above or below the organization in the supply chain, e.g., suppliers, customers.

The above list brings the most recurrently mentioned TT types in the literature, but it is not an exhaustive one. Furthermore, these types are not mutually exclusive, as a transfer might fall into two or more types, e.g. an American company may transfer a technology from a research institute or university in the UK to be used 
in one of its business units in America. In that case, the transfer would be external (different entities involved), from university to the private sector, and international.

The next section dives a little deeper into two of these types: internal transfer and university transfer - the former because it is the main focus of this study and the latter because it is extensively discussed in the literature.

\section{Internal Technology Transfer}

Internal technology transfer happens inside one organization. As LeonardBarton and Sinha put it, it is "the implementation of technical systems developed and disseminated to operational subunits within a single organization" [37, p. 1125]. Nobelius highlights the importance of having a formal process in place to take care of internal technology transfer. According to the author, bringing technology research into products with commercial viability can be very painful, and internal technology process is essential in this process [1]. Stimulating internal technology transfer would not only enhance the chances of R\&D success, but as Granstrand and Oskarsson say, it will also help the organization in achieving technology diversification [85]. Although there are disagreements in the literature (as will be discussed later), some authors believe that the internal technology transfer process should be designed and dealt with on a case-by-case basis. Magnusson and Johansson argue that the process would be better conducted based on a contingency framework, rather than having a pre-established standardized process [9]. 
In a study to develop an internal technology transfer conceptual model, Malik identifies two sets of important criteria for this process. The author called it the 'likely to inhibit' factors and the 'likely to help' factors, as shown below [38]:

- Inhibiting factors [38]
- No interest in the project
○ "Not-invented-here" syndrome
$\circ$ Lack of people
$\circ \quad$ No perceived benefit
○ Lack of trust
○ Lack of training
$\bigcirc \quad$ Language barriers
○ New technology threats

- Helping factors [38]
- Adequate resources
○ Market pull
- Communication skills
- Familiarity with technology
- Willingness to transfer staff
- Culture of trust
○ Motivation 


\section{University Technology Transfer}

University technology transfer, especially from an economic and country development standpoint, is very important. According to Heinzl et al., universities are vital to the National Innovation System, because they provide diverse and high quality knowledge while disseminating good practices, know-how, and competency [22]. The author further states that a more efficient technology transfer process in universities would mean more jobs and wealth for its region and country [22]. Previous studies have tried to identify the steps involved in the process of transferring technologies from universities [24], [63]. The models depict the disclosure of the invention made in academia, followed by the protection of the intellectual rights. Also, they include the reach out effort to interested parties, the negotiation to transfer, and the appropriate mechanism to do so.

In a good summary, Chen et al. summarize the main themes in the literature when it comes to university TT: Government policy and national innovation systems; university-operated enterprises; university science parks and spin-offs; universityindustry linkages; TTO's; and university patenting and licensing [62]. In 1991, Mitchell was already noticing a change in how universities dealt with technology transfer. This time period was the beginning of the technology transfer offices (TTO's) [86]. In 1998, however, Mejia was arguing that the majority of transfers from universities was still done by publications, and a stronger linkage between university and industry was needed [39]. In another comprehensive study on university technology transfer, Siegel et al. create a series of propositions after analyzing the literature, pointing to characteristics and challenges such as providing more rewards 
for researchers, providing more and better resources for TTO's, changing the mentality of university researchers, management and marketing efforts on TTO's, more flexibility on the university's side, and more formal and constant interactions between industries and universities [63].

\section{TT Mechanisms}

As already mentioned, mechanism is the vehicle used to transfer the technology. Table 2 lists the most mentioned TT mechanisms in the literature:

Table 2 - TT Mechanisms

\begin{tabular}{|c|c|}
\hline \multicolumn{2}{|l|}{ Technology Transfer Mechanisms } \\
\hline Mechanisms & Reference \\
\hline Contract Research & {$[22]$} \\
\hline Foreign Direct Investment (FDI) & {$[52]$} \\
\hline Further Development & {$[44]$} \\
\hline Internal Start-Ups & {$[44]$} \\
\hline Joint-Venture R\&D & {$[22][35][24]$} \\
\hline Licensing & {$[22][52][24]$} \\
\hline Meetings & [24] \\
\hline Mobility Scheme & {$[22]$} \\
\hline Monitoring of Activities of the Science Base & {$[22]$} \\
\hline Movement of Personnel & {$[52]$} \\
\hline Publications & {$[24]$} \\
\hline Regional Technology Centers & [35] \\
\hline Reverse Engineering & {$[52]$} \\
\hline Science Parks & {$[35]$} \\
\hline
\end{tabular}




\begin{tabular}{|c|c|}
\hline Spin-Offs & {$[22][24]$} \\
\hline
\end{tabular}

Contract research are contractual arrangements between the technology developer and the technology recipient. It sets the basics of the transfer-who is involved, what will be transferred, and how [22]. Foreign direct investments (FDI) are characterized when an organization makes an investment to own another organization in a foreign country. According to De la Tour, FDI in developing countries are "...carried out to benefit of cheap labor, they hire local work-force to which the know-how is then transferred" [52]. Further development happens when a technology is not completely ready yet, and follow-on research is needed. Also, further development is necessary when a technology is not significant enough for the industry willing to deploy it, or when the technology does not result in competitive advantage for the organization [44]. Transfer by internal start-ups happen when a technology generated within an existing organization is explored by a new company with relative independence from the original organization. According to Festel, internal start-ups would be fast and flexible enough to speed-up the transfer process [44]. Joint-venture R\&D happens when two or more organizations share the costs, risks, and potential benefits of a technology development project. Seaton and CordeyHayes argue that joint-venture $R \& D$ efforts have the ability to lower the risks of development, and make the transfer less painful [35]. Licensing happens when an organization authorizes another to use and/or commercialize a technology, without transferring the ownership, and it usually involves the payment of license fees. According to De la Tour, licensing is the most obvious mechanism of technology 
transfer [52]. Meetings can be used as a mechanism to pass information along to other parties. As Rogers et al. put it, "meetings involve person-to-person interaction through which technical information is exchanged" [24]. Mobility Scheme comprises the movement of people, and, this movement can be temporary. Heinzl et al. mention some of these movements: Professors during sabbaticals, summer internships, and temporary movement of personnel within and between organizations, among others [22]. Monitoring of activities of the science base happens when an organization is attentive to the flow of knowledge in its field. Searches on academic article and patents databases, participation in conferences, and industry forums would be some of the activities involved [22]. Movement of personnel is similar to the mobility scheme. It involves sending workers along with the technology in order to ease the transfer, and these movements may not be temporary, as in the case of mobility schemes. De La tour argues that these movements can be essential for a successful transfer [52]. Publications in journals and magazines are a way of transferring knowledge and technology, which are used extensively in academia. However common it is in university transfer, Rogers et al. caution that this is not the best mechanism for transfer, as usually articles are written in language following academia standards and not very easily understood by practitioners [24]. According to Seaton and Cordey-Hayes, regional technology centers are used in the UK as a midpoint between technology donors and recipients-these entities have a database of technologies available for transfer, and help the involved stakeholders in the process, aiming to improve the success of the transfer [35]. Reverse engineering consists of analyzing a product or technology in its final form, and trying to understand its 
components and sub-systems. De la Tour suggests reverse engineering as a transfer mechanism for companies that import products [52]. Science parks and incubators support and protect start-ups in their initial stages. Seaton and Cordey-Hayes argue that by providing this support science parks would help in the transfer of technologies [35]. Spin-offs are usually referred to as companies that are born out of universities where professors and/or students decide to explore their inventions on their own, as a company [22].

\section{TT Methods}

Both quantitative and qualitative methods are found in the literature. Quantitative methods would include methods and tools focusing on mathematical and statistical models, plus Multi-Criteria Decision-Making Models (MCDM), such as Analytical Hierarchical Process (AHP) and Hierarchical Decision Modeling (HDM). According to Khabiri et al., qualitative methods define activities of those who are involved in the process, and elicit factors and issues that may influence the success and effectiveness of a TT project. On the other hand, quantitative methods would quantify parameters and analyze them. Also, they try to minimize incompatibilities between donors and recipients [53].

Table 3 summarizes some of the sources found in the literature that use and/or mention methods for technology transfer: 
Table 3 - TT Method Types

\begin{tabular}{|c|c|}
\hline \multicolumn{2}{|c|}{ Technology Transfer Method Types } \\
\hline Type & References \\
\hline Quantitative & {$[6],[25],[36],[51],[59],[61],[87]-[90]$} \\
\hline Qualitative & {$[2]-[4],[7],[22],[23],[34],[35],[38],[41]$,} \\
& {$[54],[63]$} \\
\hline
\end{tabular}

Table 4 summarizes some of the sources found in the literature that use and/or mention specific methods and tools for technology transfer:

Table 4 - TT Methods and Tools

\begin{tabular}{|c|c|c|}
\hline \multicolumn{3}{|c|}{ Technology Transfer Methods and Tools } \\
\hline Method & Type & Reference \\
\hline AHP & Quantitative & {$[90][91]$} \\
\hline Decision-Model & Qualitative & {$[2],[22],[34]$} \\
\hline Fuzzy-Set Theory & Quantitative & {$[90]$} \\
\hline HDM & Quantitative & {$[6],[19]$} \\
\hline Interviews & Qualitative & {$[63]$} \\
\hline Mathematical Models & Quantitative & {$[87],[89]$} \\
\hline Other Types of MCDM & Qualitative & {$[49],[51]$} \\
\hline Nonlinear Differential Equation & Quantitative & {$[25]$} \\
\hline Scenario Analysis & Qualitative & {$[25]$} \\
\hline Maturity Scale & Qualitative & {$[91]$} \\
\hline Social Network Analysis & Qualitative & {$[92]$} \\
\hline
\end{tabular}

As shown in Table 4, subjective models are, by far, the most dominant way of dealing with technology transfer. Within the subjective models, Multi-Criteria 
Decision-Making (MCDM) methods are the most frequently used. As aforementioned, technology transfer is a complex, multi-faceted, and multi-disciplinary effort. As such, it seems that MCDM models are the most appropriate methods to deal with technology transfer since they can approach the problem from several different perspectives at the same time. AHP [90], [91] and HDM [6], [19] are not the only models used, but also decision models based on donor/recipient criteria [2], [22], [34]; manufacturing strategy [41]; contingent effectiveness [3], [7]; broadcasting [38]; multi constituency [35]; and climate-friendly technology transfer [4].

\section{TT Application Areas}

Technology transfer models developed in the literature have been applied to a myriad of sectors, from nanotechnologies to aerospace. Table 5 brings a list of some of the application areas found in the literature:

Table 5 - Technology Transfer Application Areas

\begin{tabular}{|c|c|}
\hline \multicolumn{2}{|c|}{ Technology Transfer Application Areas } \\
\hline Area & References \\
\hline Aerospace & {$[25]$} \\
\hline Climate Change & {$[88],[93]$} \\
\hline Construction & {$[51]$} \\
\hline Electric Components & {$[38]$} \\
\hline Energy & {$[4]-[6],[52],[54],[94]$} \\
\hline Information Technology (IT) & {$[49],[87]$} \\
\hline Nanotechnologies & {$[7]$} \\
\hline Semiconductors & {$[50]$} \\
\hline
\end{tabular}




\begin{tabular}{|l|c|}
\hline Steel Production & {$[95]$} \\
\hline
\end{tabular}

\section{TT Complexities, Challenges, and Gaps}

Technology transfer, although having been the subject of research for over 45 years, still presents a fair share of challenges to both practitioners and researchers, due to its fuzziness and complexity. It is still unclear how to deal with the process, and how to manage and conduct it in a proper way. Festel mentions the research gap that exists in the transfer of research and development outcomes to the successful commercialization of those outcomes [44]. As it was discussed, the very definition of TT is not consensual as it varies significantly from discipline to discipline. According to Bozeman, TT involves a very large set of players, activities, and interests, making it burdensome [3]. Still according to the same author, TT is a very complex and risky process. It is very time consuming, and usually does not produce the expected results [3]. It is not clear where and when TT starts and ends, and concepts like prototypes and proof of concept are not clear either. Most of the time, prototypes are developed without any concern for user requirements, and still, they are used as a basis for the final product [60]. Literature suggests the technology transfer process and efforts are challenging, and also suggest that organizations should strongly support and care about the process should they want to obtain good results. Isaacs and Tang say that TT is a contact sport, as the transfer is not done by materials or reports, but is done by people. There should be a high commitment and support for the TT process [60]. 
Concerning university technology transfer, Landry et al. perceive a research gap to be filled. The authors argue that when it comes to TTO's, there is a need to conduct a demand-side perspective study to understand how TTO clients perceive those organizations [40]. Some of the questions would be: Do they appreciate its services? What do they like or dislike about it? How can TTO's be more helpful and effective?

As for the internal technology transfer type, some authors also identify a need for further investigation and research. Malik states that more studies should be done on intra-firm TT to understand it, since the majority of research is done on international and external transfer processes [38]. Magnusson and Johansson also argue that more research on internal technology transfer is needed [9].

\section{TT Relationship with Other Fields and Concepts}

By analyzing the literature, one can clearly notice that technology transfer, as a field, overlaps with several other fields and concepts. The section below intends to shed a light on those overlaps and explain how these interactions happen.

\section{Commercialization}

To the vast majority of scholars, technology transfer is intimately related to the concept of commercialization. In the context of TT, commercialization would mean successfully bringing to the market a product and/or service developed during an R\&D project. A solid technology transfer process would enable the successful commercialization of new technologies [36]. Improving the technology transfer process and partnerships would improve the commercialization results [7]. 
Innovation commercialization would be helped by, and go beyond, technology transfer [22]. Technology transfer would bridge the gap between R\&D and commercialization [50]. If technology transfer is better thought of and understood, chances of successful technology commercialization increase [6]. In summary, scholars agree that technology transfer would enable, improve, or have commercialization efforts as part of the process. Table 6 brings a list of studies that mention the relationship.

Table 6 - Relationship between Technology Transfer and Commercialization

\begin{tabular}{|c|c|}
\hline \multicolumn{2}{|c|}{ Relationship between Technology Transfer and Commercialization } \\
\hline Studies Mentioning the & {$[3],[6],[7],[22]-[24],[36],[40],[50],[59],[61]-[63],[86]$} \\
Relationship & {$[92],[96]$} \\
\hline
\end{tabular}

\section{Policy-Making}

A myriad of studies touches upon policy issues when dealing with technology transfer since the transfer from the public sector to the private is one of the biggest branches of the field. Furthermore, in understanding how crucial the technology transfer process is for the overall technological development of a region/country, public authorities venture to try to boost and promote the process. In 1977, BarZakay published a study where the primary objective was to create policy recommendations to enhance and improve the technology transfer from the military sector to the civilian sector in Israel [34]. In analyzing international technology transfer, Bommer at al. mention government policies as a critical factor to be taken into consideration [41]. In analyzing technology transfer in the Solar PV industry, 
Zhang and Gallagher state that government policies would be one of the main drivers [94]. Worrell et al. argue that policies can heavily influence the technology transfer process [5]. Lai and Tsai state "government's policy is always a crucial factor in influencing TT" [90, p. 74]. Table 7 brings a list of studies that mention the relationship.

Table 7 - Relationship between Technology Transfer and Policy-Making

\begin{tabular}{|c|c|}
\hline \multicolumn{2}{|c|}{ Relationship Between Technology Transfer and Policy-Making } \\
\hline Studies Mentioning the & [2]-[7], [22]-[25], [34]-[36], [40], [41], [48], [49], [51], [52], [54], \\
Relationship & [59], [62], [63], [87], [90], [92]-[96] \\
\hline
\end{tabular}

\section{Technology Assessment}

Technology assessment is another concept/practice that has an intimate relationship with technology transfer. It is critical to assess and understand the technology one wants to transfer irrespective of the type of transfer or entities involved. Assessing the technology under different perspectives is always a part of the TT process, whether it is a quantitative process or a qualitative process. Worrell et al. name technology assessment one of the stages of TT, followed by agreement, implementation, evaluation and adaptation, and repetition [5]. Talaei et al. used AHP to assess technologies before recommending policies to transfer energy technologies to developed countries [4]. Similarly, Liu uses technology assessment as part of the transfer process when dealing with aircraft engine technologies [25]. Bar-Zakay argues that technology assessment should be conducted before choosing 
technologies to be transferred, as to anticipate the changes and problems a society may face after the new technology is transferred and deployed [2]. As part of their transfer process for the steel industry, Okazaki and Yamaguchi assess the technology and its barriers [95]. Table 8 brings a list of studies that mention the relationship.

Table 8 - Relationship Between Technology Transfer and Technology Assessment

\begin{tabular}{|c|c|}
\hline \multicolumn{2}{|c|}{ Relationship Between Technology Transfer and Technology Assessment } \\
\hline Studies Mentioning the Relationship & [2], [4], [5], [25], [36], [87], [95] \\
\hline
\end{tabular}

\section{Technology Development}

There is still, to a degree, a debate in the literature about when technology transfer efforts should start, or even if an organization should or not have a formal TT process in place. Some think that TT should start only after the technology development project is done. Others say the TT process should be conducted in parallel. This debate will be further discussed in later sections, but the overlap and interactions between technology development and technology transfer are obvious.

Some authors highlight the interrelationship between public policies promoting technology development and promoting technology transfer [3], [62], [95], [96]. Other authors argue that different TT models are needed for technologies that are at different development stages [36], [61]. Estep links the TT process to the very early stages of technology development, bringing it to the assessment and selection of research proposals [6]. For university TT, Bozeman et al. argue that royalties are greater when more developed technologies are transferred [7]. From an 
economic standpoint, Siegel et al. stated that when industry partners with academia for TT purposes, the whole sector experiences a greater technological development [63]. Table 9 brings a list of studies that mention the relationship.

Table 9 - Relationship Between Technology Transfer and Technology Development

\begin{tabular}{|l|l|}
\hline \multicolumn{2}{|c|}{ Relationship Between Technology Transfer and Technology Development } \\
\hline Studies Mentioning the Relationship & {$[3],[6],[7],[19],[36],[61]-[63],[95],[96]$} \\
\hline
\end{tabular}

\section{Product Development}

As already mentioned, there are advantages in filling the gap between technology development and product development by increasing an organization's technology transfer capabilities. The new product development process is likely to go smoother if the technology transfer was adequately done. There are, however, other relationships between the technology transfer process and the actual product development process. Jugend and Silva say that the technology transfer process, among other factors, is vital in having effective new product development projects [97]. As Spann et al. put it, there is a technology transfer component within the product development process [61]. Bommer et al. argue that, for long and costly product development projects, strategic alliances and transfer skills are necessary [41]. In a research on transfer of energy-efficient technologies, Worrell et al. state that better technology transfer skill scan lead to better and more environmentally-sound products [5]. Nobelius suggests that the transfer to the market should be thought of before the new product development project is over. According to the author, usually 
organizations only think about the transfer after the NPD project is done, and that would be a cause of delays [1]. Table 10 brings a list of studies that mention the relationship.

Table 10 - Relationship Between Technology Transfer and Product Development

\begin{tabular}{|l|l|}
\hline \multicolumn{2}{|c|}{ Relationship Between Technology Transfer and Product Development } \\
\hline Studies mentioning the relationship & [1], [5], [7], [37], [41], [61], [63], [97] \\
\hline
\end{tabular}

\section{Technology Forecasting}

In the literature, the relationship between technology transfer and technology forecasting is not as strong and clear as in other cases, but some authors dwell on the subject. The reasoning is similar to that of the technology assessment concept-the more information on the technology, the better for planning the transfer. Thus, if a technology forecasting assessment provides an organization with valuable insights on what directions the technology is taking or on what changes are going to be there as a result of the technology application, the better the transfer can be planned and conducted. For the most part, it is said that technology forecasting assessments are, to some extent, situated within the technology transfer efforts [2], [25], [36], [96]. Bar-Zakay is more straight forward, and argues that forecasting skills are necessary for an organization to be successful at transferring technologies [34]. Table 11 brings a list of studies that mention the relationship. 
Table 11 - Relationship Between Technology Transfer and Technology Forecasting

Relationship Between Technology Transfer and Technology Forecasting

Studies Mentioning the Relationship

[2], [25], [34], [36], [96]

\section{Technology Maturity}

As in the case of technology forecasting, scholars do not highlight a very strong relationship between technology transfer and technology maturity. However, some interesting insights can be retrieved from the literature. As a general rule, scholars say that technology maturity / readiness would serve as one of the criteria to be considered during the transfer process. Technologies that are more ready are easier to transfer [44], [96]. During the transfer assessment of technologies, the maturity is an input [4], [23], [36], [87]. In describing the technology platforms implemented in Russia (policy instruments to boost technology development and transfer), Proskuryakova states that technology readiness was one of the criteria considered [96]. Table 12 brings a list of studies that mention the relationship.

Table 12 - Relationship Between Technology Transfer and Technology Maturity

\begin{tabular}{|c|c|}
\hline \multicolumn{2}{|c|}{ Relationship Between Technology Transfer and Technology Maturity } \\
\hline Studies Mentioning the Relationship & [4], [23], [36], [44], [87], [96] \\
\hline
\end{tabular}

\section{Open Innovation}

As the importance of open innovation grows, the relationship between that concept and technology transfer becomes more evident since one needs good technology and knowledge transfer skills in order to take advantage of open 
innovation. Confirming that idea, Carayannis et al. state that "open innovation is knowledge diffusion and recombination, producing the 'seed corn' of tomorrow's breakthroughs" [31, p. 435]. For modern organizations, open innovation is now part of their strategic planning [98], and the concept of open innovation breaks the old paradigm of developing new technologies, products, and processes using only internal expertise. The concept is predicated on the idea that organizations do not have all the expertise they need to thrive in such an aggressively competitive world, and therefore they need to branch out and use as many intellectual resources as they can, and from various sources. Open innovation incurs in utilizing resources that are external to the organization to create value. As Henry Chesbrough defines it, "open innovation is the use of purposive inflows and outflows of knowledge to accelerate internal innovation, and expand the markets for external use of innovation, respectively" [99, p. 1]. As aforementioned, good communication and knowledge transfer skills are essential for any open innovation effort. Carayannis et al. list seven of the research streams or characteristics of open innovation: Integration of external partners, partnering and alliances, open innovation processes, open innovation tools, open trade of intellectual property, open business models, open innovation culture [98]. To a greater or lesser degree, all those characteristics are related to technology transfer aspects, most importantly the ability to acquire, communicate and share knowledge, and the ability of a set of successful collaborative R\&D partnerships, as evidenced by the discussion around precompetitive, competitive and coopetitive technology transfer types [55]. Table 13 brings a list of studies that mention the relationship between open innovation and knowledge/technology transfer. 
Table 13 - Relationship Between Technology Transfer and Technology Maturity

\begin{tabular}{|l|l|}
\hline \multicolumn{2}{|c|}{ Relationship Between Technology Transfer and Open Innovation } \\
\hline Studies Mentioning the Relationship & [7], [22], [30], [31], [55], [97], [98], [100]-[102] \\
\hline
\end{tabular}

\section{Technology Transfer/Commercialization in the Energy Sector}

The concepts of technology transfer and technology commercialization are intertwined. Researchers in the 1970s used to regard technology transfer solely as the movement of technology from one context or organization to another [2], [34]. Over time, the TT (technology transfer) concept and understanding were expanded to include commercialization aspects. Recent researchers, especially from the late 2000s on, have been regarding and discussing technology commercialization as technology transfer [22]-[24]. The commercialization happens when a technology is developed and brought into the market as a product or service. In the words of Balachandra and Reddy, "commercialization is the total process of moving a technology from the concept stage, to the production of a product and from there, to market acceptance and use" [103, p. 6]. For the purposes of this explanation, the terms technology transfer and technology commercialization will be used interchangeably.

The commercialization or the transfer processes start with R\&D to develop the technology. R\&D can be performed internally by using its own capabilities, or can be performed externally through licensing or technology acquisition., It can also be performed by a mixture of internal and external $R \& D$, through joint-projects, partnerships, collaborative development, and open innovation initiatives. Each 
organization will analyze its options, and focus on the ones that are more adequate according to its capabilities and objectives. It is certain, however, that the right approach is to make use of all available sources of knowledge and expertise to innovate, thereby avoiding missed opportunities. According to Hess and Siegwart, the organization that has both internal and external R\&D efforts and that balances both breakthrough and incremental innovation, is likely to better control the risks involved in the projects while also maintaining its absorptive capacity [104]. Hung et al. complement this line of thought by stating that technology commercialization depends on the firm's capabilities to be successful [105]. The same authors also affirm that technology commercialization would be beneficial for boosting the demand in a particular sector, thus boosting technology development and advancements [105]. Furthermore, the importance of technology commercialization is highlighted when Worrel et al. suggest that tech transfer and commercialization should be an integral part of an organization's strategy [5]. Similarly, Hess and Siegwart conclude that technology transfer and commercialization capabilities are essential to any organization that wants to be competitive in the market [104].

In order for a technology to be successfully commercialized, it has to be brought to the market and to be adopted by its consumers. Although the criteria and process through which a technology is adopted might change from sector to sector (and possibly from person to person), a few general steps are discussed in the literature, aiming to inform technology developers throughout the design and development of technologies and products. As explained by Balachandra and Reddy, the judging process of a technology would go through five steps, namely trialability, 
observability, relative advantage, complexity, and compatibility [103]. In sum, a customer would adopt a technology after trying it out or testing it, observing the results of the trial, comparing the advantages against other options, judging how complex it is, and how compatible it is with his/her style and way of doing things. At the other end, technology developers should be keen in measuring their commercialization performance. In order to do so, researchers suggest the use of metrics. Carayannis and Alexander list several metrics to be taken into account, divided into four categories: Input metrics, intermediate metrics, short-term metrics and long-term metrics. Time and money spent on technology transfer would be examples of input metrics, number of invention disclosures, and papers published would be examples of intermediate metrics, patents and licenses would be examples of short-term metrics, while ROI and market-share would be examples of long-term metrics [106]. It is important to note that the number of patents is listed as a shortterm metric, not as a long-term one. This might seem, at first, a little dissonant regarding the body of knowledge in technology transfer since intellectual property issues and aspects are a commonplace and considered important for technology transfer and commercialization [22], [24], [38], [63]. Nonetheless, to regard patents (and IPs in general) as one of the criteria but not as the most important criterion is valid and logical. Researchers agree with the idea that patents are not a positive result in and of themselves, but rather they are a mechanism to protect technologies from competitors so the organization can market them and achieve the real positive results that matter. Rubenstein, for example, argues that some organizations focus excessively on intellectual property and relegate the real outputs of technology 
development—-such as new products that will be adopted by consumers and generate revenue, profit, and market-share—to a less important role [107].

Taking the literature on technology commercialization out of the general discussion and bringing it specifically to the energy sector, it is noticeable that this sector has been the subject of numerous studies. The power sector and its utilities are widely recognized as being particularly conservative in the way they do business, and this behavior is derived mostly from the fact that the sector is highly regulated, operates in a monopolistic or semi-monopolistic fashion, and concessions and contracts are long-term [108]. These characteristics can result in an unwelcoming environment for change and innovation, making electric utilities adverse to conducting research and development [108]. This reality, however, is changing. The whole sector seems to be waking up, developing new technologies, and researchers are devoting more attention to studying innovation and R\&D in the power sector. With regard to renewable sources of energy, a myriad of technology transfer and commercialization studies have been conducted [109]-[111], and more examples will be given later in this study. According to Liu and Liang, R\&D and innovation are needed in the energy sector, otherwise clean energy technologies will not be as ready and available, nor initiated as quickly and with the global reach as we expect [112]. There are several reasons why utilities are moving faster now towards innovation, chief among which is the high level of obsolescence of their infrastructure and the expectations for renewables. In the words of Hess \& Siegwart, "aggressive demands for energy savings and an aging power supply infrastructure demand real 
breakthrough innovation and radical improvements to the inspection service, to power generation and to the distribution infrastructure" [104, p. 154].

Although the power sector has come late to the "technology era" when compared to other sectors, the reality is very promising for utilities and for consumers as well. Following Hsu \& Chang, the evaluation of new energy technologies should be broadened to consider other potential products that could derive from them, as the possibilities of innovation are very good [113]. As part of the discovery process of new technologies and options, demonstration projects, and institutions to promote and manage those projects, are needed for accelerating the development of energy technologies [110]. More research will lead to more demonstrations and those, in turn, will lead to more successful transfer and commercialization of those technologies. That would not only be desirable, but also crucial for the energy sector. As Malek et al. put it, renewable energy technologies success heavily rely on demonstration and commercialization efforts [110]. Most of scholars agree that the energy technologies that could revolutionize the grid are not ready yet, and these technologies are not successful in surviving the notorious valley of death [112]. While there is still a long way to go, researchers are focused in finding paths to shorten the distance and overcome the barriers that prevent these technologies from being successfully transferred to the market. Joining efforts and sharing risks in the development process is one of the ways being praised by scholars. The partnership synergy, as explained by Manouklan et al., could help new energy technologies to cross the valley of death, and to be properly commercialized and adopted in mainstream markets [114]. Additionally, having close relationships and partnering 
with universities and research institutes could be a shortcut for those technologies [115]. As much as new research, both technical and managerial, is rising in the energy technology field (most importantly for renewables), scholars still mention the need for more and better technology transfer models for the power sector [104].

In order to have a sense of what the research landscape of technology transfer and commercialization in the energy sector looks like, the most common types and purposes of transfer are presented, as well as the application areas, mechanisms of transfer, and methodological approaches used by scholars. In some cases, it is possible to compare it to the general landscape of technology transfer research. When it comes to the type of transfer, the TT research on the energy sector is similar to the overall TT research with most of the research pieces dealing with international transfers [4], [5], [108], [109], [116]-[120]. There are also studies on university technology transfer [121], but as opposed to the overall technology transfer body of knowledge, few studies focused on university technology transfer in the energy realm. When it comes to the purpose of transfer, the literature is dominated by studies attempting to use energy technology transfers as a means to mitigate and minimize carbon emissions in developing countries due to climate change concerns [4], [5], [103], [108], [117], [120], [122], [123]. The application of these studies is mostly on renewable energy sources, as shown in Table 14. 
Table 14 - Technology Commercialization in the Energy Sector - Application Areas

\begin{tabular}{|c|c|}
\hline Application & Reference \\
\hline Batteries & {$[124]$} \\
\hline Energy Efficiency Technologies & {$[125]$} \\
\hline Military Systems & {$[126]$} \\
\hline Clean / Renewables & {$[109]-[111]$} \\
\hline Solar PV & {$[94],[119],[121]$} \\
\hline Sustainable Technologies & {$[4],[103],[108],[112],[117]$} \\
\hline Wind & {$[115],[116],[118]$} \\
\hline
\end{tabular}

The mechanisms found in the literature for the energy sector are more limited than the overall tech transfer literature with a focus on joint design, joint-ventures [108], [112], and licensing [4], [104], [109], [110], [112], [115]. The methodological approaches, however, are consistent with the general literature with heavy emphasis on multi-criteria decision making (MCDM) models, as shown in Table 15.

Table 15 - Technology Commercialization in the Energy Sector-Methods

\begin{tabular}{|c|c|}
\hline Methodological Approach & References \\
\hline AHP + TOPSIS & {$[118]$} \\
\hline HDM & {$[6],[127]$} \\
\hline Other Hierarchical Models & {$[6],[125],[127]$} \\
\hline Multiple Perspectives & {$[128]$} \\
\hline
\end{tabular}

There are numerous factors and stakeholders that compose TT and influence its outcomes. Generally speaking, there are eight different types of stakeholders: 
Government, information holders, market intermediaries, technology buyers, technology developers, technology financiers, technology owners, technology users [103]. Scholars research and discuss factors and components of technology transfer, some act as facilitators, while some act as barriers for the success of the process. As technology transfer is complex and multidisciplinary, both quantitative and qualitative factors come into play when assessing TT processes [108]. As general effectiveness factors, Bozeman lists factors such as market and economic impacts, the political impact and the opportunity costs of transferring technologies, and also the human capital involved in the process [3]. The same author, using national laboratories environments as an example, lists the transferor, transferee, the transfer object, medium, and also the demand conditions as important factors [3]. Rubenstein discusses positive and negative factors influencing the commercialization process, and divides those in early stage factors and late stage factors. Lack of funding, human resources, and training would be early stage negative factors, while lack of motivation and market knowledge would be late stage negative factors; explicit goals, technical personnel, and sufficient funding would be early stage positive factors, whereas motivation and market knowledge would be late stage positive factors [107]. Another important study identifies steps to be taken in order to conduct a successful commercialization, namely spotting and understanding the adopters; designing the item according to their needs; providing the necessary support after the transfer is done [103]. Also trying to identify necessary factors for a successful commercialization, Salwan lists, among other factors, the understanding of the user needs; feasible solutions for those needs; a close relationship between donor and 
recipient; market knowledge; support for the process [126]. Regardless of the factors that may be involved or not on the process, it is clear that the organization needs to have strategic planning in order to benefit from technology transfer [109].

When it comes to specific criteria to be taken into consideration in order to build or assess a technology commercialization process, a myriad of items are identified and discussed in the literature. A sample of what can be found in technology transfer and commercialization studies is shown in Table 16.

Table 16 - Technology Commercialization in the Energy Sector - Criteria

\begin{tabular}{|c|c|}
\hline Criteria & References \\
\hline Dedicated TTO & {$[59],[127]$} \\
\hline Geographical Proximity & {$[6],[59]$} \\
\hline Business Plan & {$[59],[111],[127]$} \\
\hline Shared Personnel & {$[59]$} \\
\hline Preliminary Manufacturability Assessment & {$[124]$} \\
\hline Safety and Environmental Assessment & {$[105],[124]$} \\
\hline Budget & {$[111],[127]$} \\
\hline Common Standards & {$[127]$} \\
\hline Communication & {$[128]$} \\
\hline Compatibility & {$[108]$} \\
\hline Complexity & {$[108]$} \\
\hline Cost of Application & {$[113]$} \\
\hline Trust Atmosphere & {$[127]$} \\
\hline Demonstrations & {$[127]$} \\
\hline Technology Working Models/Prototypes & \\
\hline
\end{tabular}




\begin{tabular}{|c|c|}
\hline Economic Analysis & {$[113],[127]$} \\
\hline Senior Management Support & {$[126],[127]$} \\
\hline Regulatory Issues & {$[126]$} \\
\hline Feasibility & {$[126]$} \\
\hline Governance & {$[111]$} \\
\hline Government Incentives & {$[127]$} \\
\hline Technology Potential & {$[126]$} \\
\hline Joint Research & {$[6],[126]$} \\
\hline Innovation Observability & {$[108]$} \\
\hline Organizational Homogeneity & {$[127]$} \\
\hline Innovation Risk & {$[6],[108]$} \\
\hline TT-dedicated Team & {$[127]$} \\
\hline Strategic Alignment & {$[111],[127]$} \\
\hline TRL Assessment & {$[124],[128]$} \\
\hline Market Assessment & {$[122],[127]$} \\
\hline
\end{tabular}

An important aspect of the technology commercialization literature in the energy sector is the strong relationship with technology policy. Whether the focus of the study is international technology transfer, or the commercialization of a technology developed at a university or national lab, there is, in the majority of cases, a connection with public policies on technology development and diffusion. Technology policy is seen as essential not only to a successful transfer, but also to incentivize the development and to boost adoption [115]. One of the most widely known and used instruments are the Cooperative Research and Development Agreements (CRADA), which are important mediums through which DoE 
(Department of Energy) laboratories can make their energy technologies reach the market [129]. The objective of CRADAS is to boost R\&D, and to help new technologies to successfully hit the market. The requirements for establishing a CRADA with DoE are, among others, to result in a benefit that is consistent with DoE's program objectives without compromising national interests and objectives, and to take actions towards the dissemination (transfer) of the collaboration outcomes [130]. Authors agree that CRADAS are a very efficient way to leverage federal funding for energy technologies and to get it effectively transferred to the market [131]. The relationship between energy technologies, policy, and legal incentives for their development and commercialization dates back to at least the 1980s. According to Perry, three of the most beneficial acts for the DoE labs were the Stevenson-Wydler Technology Innovation Act (enacted in 1980), the Bayh-Dole Act (enacted in 1980), and the Federal Technology Transfer Act (enacted in 1986) [132]. These laws were aimed at having technology transfer at the core of the labs, and at solving technology commercialization issues, such as lack of funding and intellectual property issues. For some authors, policies and governments play such a major role in energy technologies that not only policies would influence its development and transfer, but also the composition of the government itself. According to Cirone \& Urpelainen, a unified government, rather than a fractioned one, is vital should a country want to advance its energy technologies [133].

In the literature, there are some frameworks that focus on how to develop public policies for energy technologies. In the work of Worrel et al., for example, the authors suggest a four-step process for energy policy-making, namely assessment of 
technologies, which would be targeted by the policy; formal agreements between government agencies and private sector entities that will be partners in the development, and/or be the recipients of the transfer; evaluation of the users' environment and the occasional adaptation of the technology to suit their needs; and support for the recipient and repetition of the process [5]. Nevertheless, some may disagree with formal and general frameworks for policy-making. Cao et al., for instance, argue that in order for the policy to be successful and effective, it has to be customized according to each type of technology [125].

Within the policy-making studies in the energy sector, the energy-efficiency technologies and initiatives are prevalent. Scholars state that energy-efficiency is the main actor, and represents the main group of technologies for energy policy [5]. Furthermore, the development and adoption of energy-efficient technologies is burdensome and takes more time than other technologies because of several barriers still in place, thus energy policies are still very important for that group of technologies [125].

\section{R\&D and Business Strategy}

After discussing technology transfer, both in general and specifically related to the energy sector, a succinct review on the literature about aligning $R \& D$ and business strategy is presented. By shedding a light on its main characteristics, importance, challenges, and limitations, the foundations are laid to start exploring ways to use both technology transfer to tackle this issue. 


\section{General Aspects of R\&D Alignment with Business Strategy}

The first aspect to approach is to establish the relationship between technology/R\&D and business/corporate strategy. Although there is not a field of study dedicated to this issue, one can find some research (within the technology management field) that at least tangentially touches upon this issue. Chester mentions this relationship, and also states that R\&D management style is usually dependent on personal preferences from top managers [134]. Phaal et al. also highlight this relationship as something beneficial through the application of technology roadmaps [135].

This relationship exists, and needs to be cultivated because it is very important for any organization to achieve its overall objectives. Gindy et al. argue that the fastpaced technological evolution, and the fact that companies are becoming more and more dependent on technologies, has made it necessary to plan technology on a strategy level [136]. Moreover, according to Jin et al., the technology in itself is not sufficient to achieve a competitive advantage. In the author's words, "the fitness between technology strategy and corporate strategy ensures the successful deployment of a company's technological capability and enhances the company's financial performance" [137, p. 472]. Furthermore, research and development is an important part in the organizational structure of firms [138], and therefore should be considered when devising a corporate strategy. The importance of this alignment becomes even more evident when Lyne argues that including R\&D and technology in the corporate strategy makes it easier for companies to cope with otherwise devastating changes in the competitive scenario [139]. Other authors have also 
described the importance and urgency of designing business strategy with an R\&D perspective in mind, and also to keep $R \& D$ activities tightly attached to that strategy [140]-[142].

However important it is to attach $R \& D$ and business strategy together, it is not a simple task. Often each organizational unit (including R\&D) seems to have its own strategy, and tries to meet their own demands and objectives, so it is difficult to synchronize it all [143]. Although there are numerous research pieces on strategy, it is still a difficult task to make the link between technology and business strategy [140]. Technology-intensive organizations face the challenge of aligning corporate strategy and R\&D [139]. Herfert and Arbige argue that conflicting strategies lead to different approaches and actions, which in turn will lead to confusion and failure. The authors conclude that any organization would benefit tremendously if they are able to align their R\&D activities with their R\&D strategy [143].

\section{Methods and Tools for Aligning R\&D with Business Strategy}

Not many methods are described in the literature as capable of providing guidance on how to increase or control the alignment between R\&D and business strategy. Table 17 shows a summary.

Table 17 - R\&D and Business Strategy Alignment - Methods and Tools

\begin{tabular}{|c|c|c|}
\hline \multicolumn{2}{|c|}{ R\&D and Business Strategy Alignment - Methods and Tools } \\
\hline Method & Type & References \\
\hline ESTO Model & Qualitative & {$[144]$} \\
\hline Mathematical Models & Quantitative & {$[145]$} \\
\hline
\end{tabular}




\begin{tabular}{|c|c|c|}
\hline STAR + AHP & Hybrid & {$[146]$} \\
\hline Strategy-Technology Firm Fit & Qualitative & {$[147]$} \\
Audit & & {$[137]$} \\
\hline Structural Equation Modeling & Quantitative & {$[139]$} \\
\hline Voice of Technology (VOT) & Qualitative & {$[135],[136],[138],[140]-$} \\
\hline Roadmapping & Qualitative & {$[142],[146]$} \\
\hline
\end{tabular}

Herfert and Arbige mention an approach, Business Integration Team (BIT), that was applied to deal with this issue [143]. This approach is based on a multifunctional team consisting of high-level managers. These individuals have both the knowledge about the organization's strategy, and the influential power to steer the organization in the right direction. Another approach that has the potential to mitigate the issue is portfolio management. If portfolio development aspects are taken into account when designing business strategy, the whole project portfolio tends to better reflect that strategy [138]. Having a good portfolio management would also increase the chances of better assessing and decision making about which R\&D proposals to select [145].

The most recurrently mentioned method to deal with the alignment of R\&D and business strategy is roadmapping. Technology roadmaps can communicate and present strategic decisions for the whole organization [138], [148]. Technology roadmaps serve not only for technology planning, but also to align technology and business strategy [136], [149]. Roadmapping reduces the risk of technology 
development while enhancing the chances of accomplishing the organization goals [146].

\section{Gaps on the R\&D Alignment with Business Strategy}

There is no consensus, across the literature, on how to align R\&D and technology with business strategy. The limitations and gaps on the research are evident. The following is a presentation and discussion on some of these limitations and gaps.

In a very interesting study, Bosch-Sijtsema and Bosch created R\&D and innovation management strategies as options, based on corporate strategy decisions. However, for the most part, there are no clear criteria or guidelines to take into consideration when choosing between these options. Also, these strategies are black boxes that not only do not assess an organization's technology transfer capabilities, but also do not recommend any action or ways as to improve these capabilities [144]. Moreover, the study does not offer any assessment of the link between R\&D and strategy, and the strategies created do not seem to be easily applicable in a real-world situation [144]. One study in particular has identified four major criteria that would be necessary to align R\&D with strategy, but those four criteria are not sufficient to ensure the alignment, as the authors acknowledge, and no recommendations or suggestions are made as to enhance the organization's capabilities [137]. Another study developed a methodology that would help with the R\&D management by enhancing the organization's external awareness, and by the way the organization 
approaches emerging technologies, but it would not be very helpful after decisions are made regarding which emerging technologies to invest in [139].

Most of the approaches described in the literature (especially portfolio management and TRM) would help in the strategic adherence at the front-end. However, not only would the corporate strategy itself change during the technology development, but also the R\&D project (due to its fuzziness and exploratory nature) might end up by going through different paths and producing different results when compared to what it was planned or expected at the front-end. Therefore, there is a need to assess and ensure strategic adherence throughout the whole technology development cycle, and the whole R\&D project. A roadmapping-based methodology has been used to align R\&D strategies and business needs, but it does not offer alignment monitoring during or after the project's completion [136]. Roadmapping has been used in several occasions as a means to align R\&D with strategy [136], [140]-[142], [146], [148]. However useful and effective roadmapping may be while selecting projects and managing $\mathrm{R} \& \mathrm{D}$ at the front-end, it does not offer support in later stages of technology development. Other approaches and methodologies have also been used, such as portfolio management [145] and strategy-technology firm fit audit [147], all of which are also focusing on the front-end part of R\&D management.

The lack of managerial tools and processes to ensure the alignment between technology and business is evidenced in the literature. Loyarte et al. argue that technology is seen as a strategic asset since the 1980s. Nonetheless, in the words of the authors, "the two key problems are how to link technology strategy and corporate/business strategy and the identification of the decision categories in 
technology strategy" [140, p. 475]. The authors also acknowledge the usefulness of roadmaps to select the right technologies, but warn that there is a need for additional tools to validate technologies after that [140]. In some strategic planning models, as in the one Gama and Guemes-Castorena put together [138], technology roadmapping and portfolio planning are present, but there is no process to ensure R\&D outcomes will fulfill the corporate strategy. Dealing more specifically with internal R\&D, BoschSijtsema and Bosch argue that there is a need for more insights about linking the innovation ecosystem and internal technology development, and that researchers should be focusing on this issue [144].

\section{Strategic Technology Control - A Process}

After going through the concepts and research landscape of technology transfer, and the alignment of technology and business strategy, an argument is now made in favor of a process to promote the strategic control of technologies, aiming to keep the alignment of technologies with business strategy from the front-end until the application and deployment of the technology. As it was discussed in the last section, some methodologies (most importantly roadmapping) bring a good solution for the alignment issue at the front-end stage. Nonetheless, it was also discussed that the alignment at the front-end does not guarantee the alignment throughout and post-technology development. At least to some extent, a continuous and more thorough technology transfer process, with strong contributions from the technology assessment field, would mitigate this issue. 
This latent capacity of a thorough technology transfer process is observed in the literature. Nobelius suggests that a better technology transfer process can lead to a better alignment of R\&D and business applications / business strategy. According to the author, the alignment of $R \& D$ and strategy is present when the research is perceived to be relevant, its results are perceived to be ready, and it has a strong coupling with technology and product strategy [1].

A better technology transfer process would also enhance the integration of R\&D and new product development (NPD). Several authors point out the difficulties in integrating R\&D and NPD [9], [97], [150]. Although the technology transfer problem is usually framed in terms of bringing a developed product to the market (or to other organization or whatever it is), there is a gap separating the technology development process and the product development process. Authors say it is not easy to align the research and development efforts and projects with the business needs for new products. Explaining the relationship between technology development and NPD, Nobelius argues that by managing TT properly, an organization can spot the perfect timing to transfer technologies from the first process to the second. Also, the author states that delays in the NPD process, which can cause serious financial hurdles, are often caused by failures in the transfer process [1].

The lack of integration between $R \& D$ and commercialization is another issue a better technology transfer process could address. Festel indicates that there is a gap between research and development efforts, and the commercialization of its outcomes. Among the reasons, the author lists the failure of maturing technologies and establishing solid IP rights. Also dwelling on the alignment of R\&D and business 
strategy, the author also argues that the further development of technologies is a good way of achieving the alignment [44].

Having a thorough and continuous TT process in place would be the first step in addressing these gaps. Nevertheless, some scholars disagree and regard a continuous TT process as an ineffective and wasteful solution. These authors regard TT as a one-time event that should be taken care of when the transferrable item is ready [151]-[153]. Magnusson and Johansson argue that, instead of having a standard process in place, organizations should design, organize, and implement their internal TT processes on a case-by-case basis, which they call a contingency framework. By doing that, organizations would be flexible and able to better respond to changes due to different situations (transfer time, type of technology, etc...) [9]. Although a certain level of flexibility should be maintained in order for the process to accommodate different settings and situations, a standardized technology transfer process would offer much more benefits than hurdles. By ways of standardizing the process, the organization settles the general framework through which technology transfer activities are conducted, e.g., TT team, data to be collected, organizational structure, communication, strategic fit, benefit management, etc... Moreover, having a standardized process in place does not mean the organization will not be able to respond to contingencies. On the contrary, provided efficient communication and a flexible organizational culture exist, the process can be adjusted to fit the requirements of any specific situation, technology, recipient, or donor. By doing that, the organization takes advantage of the "common language" that is repeatability created by the standardized process, and also quickly and effectively responds to 
whatever contingency there is. Robbins and Milliken argue that an organization is likely to have poor effectiveness in transferring technologies if it regards the process as a one-time event only, rather than having a structured process in place [8], and Nobelius strongly advocates in favor of technology transfer as a continuous effort, as opposed to viewing TT as a one-time event [1]. Leonard and Sinha list several technical and human factors that make the technology transfer process difficult [37]. This reasoning augments the validity of the argument in favor of a continuous technology transfer process. For them, one of the most important factors that affect the fate of a transfer is communication. Users and developers should be constantly communicating and exchanging ideas if the transfer is to be successful. The authors go on to say that changes are essential for a successful transfer-either the technology will have to be adapted to the people and environment in which it will be applied, or to the contrary, people and environment would need to adapt in order to fully take advantage of the new technology [37]. Figure 2 illustrates the position of a continuous technology transfer process among other managerial processes. 


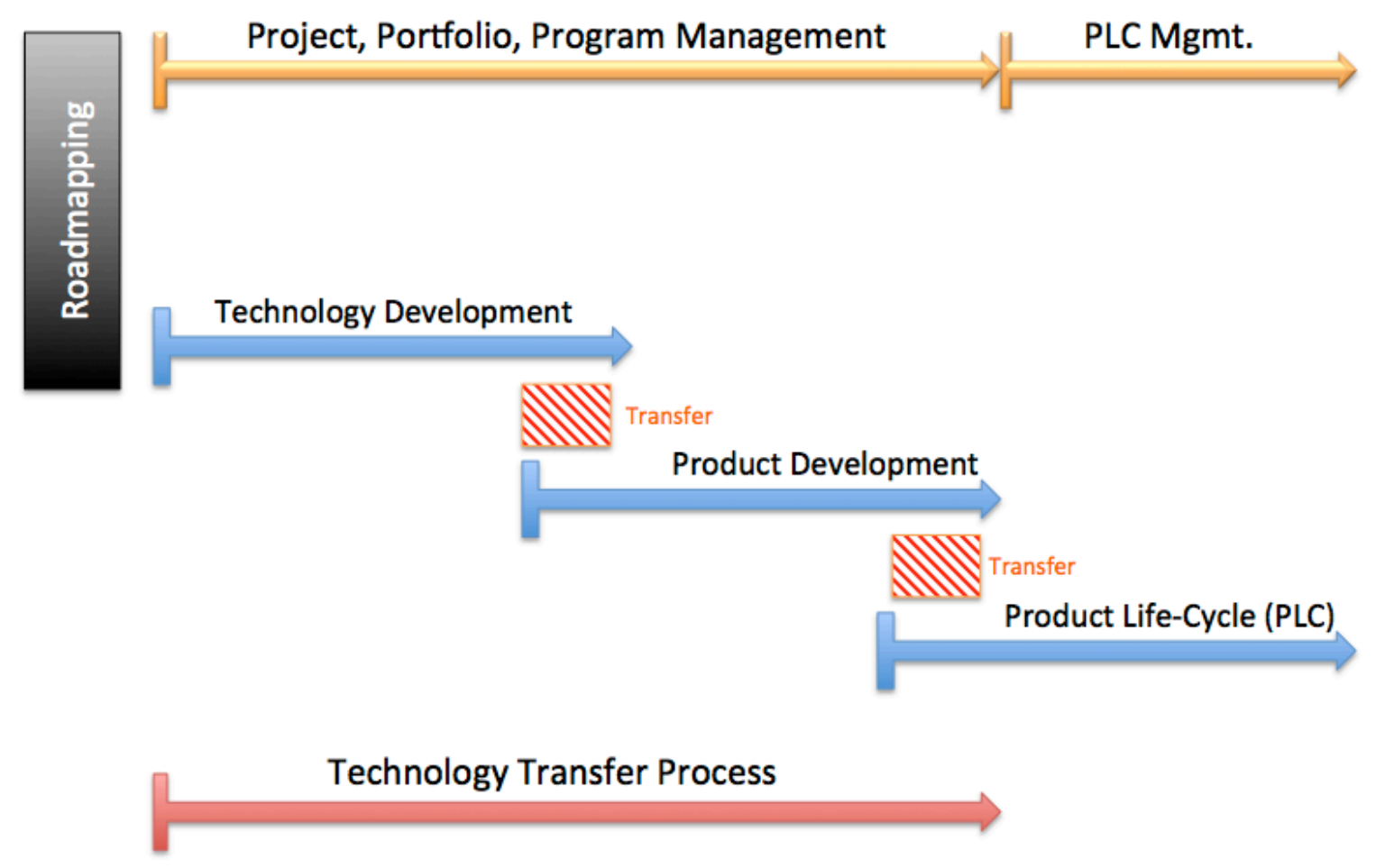

Figure 2 - Continuous TT Process

As it was discussed in earlier chapters, the roadmapping process is a powerful tool to strategically align technologies in the front-end, but it is not effective further along the technology development. Technology transfer, as a continuous process, could serve as a complement to roadmapping, assessing the technology from different perspectives at different points in the development cycle, helping thus to ensure the strategic alignment. Strategic planning activities set the strategy for the organization, and processes such as roadmapping guarantee that $R \& D$ activities are pointed in the right direction. An additional process is then necessary to make sure R\&D does not go off-track and miss business goals. Technology transfer as a continuous process could be that addition. Figure 3 illustrates this concept. 


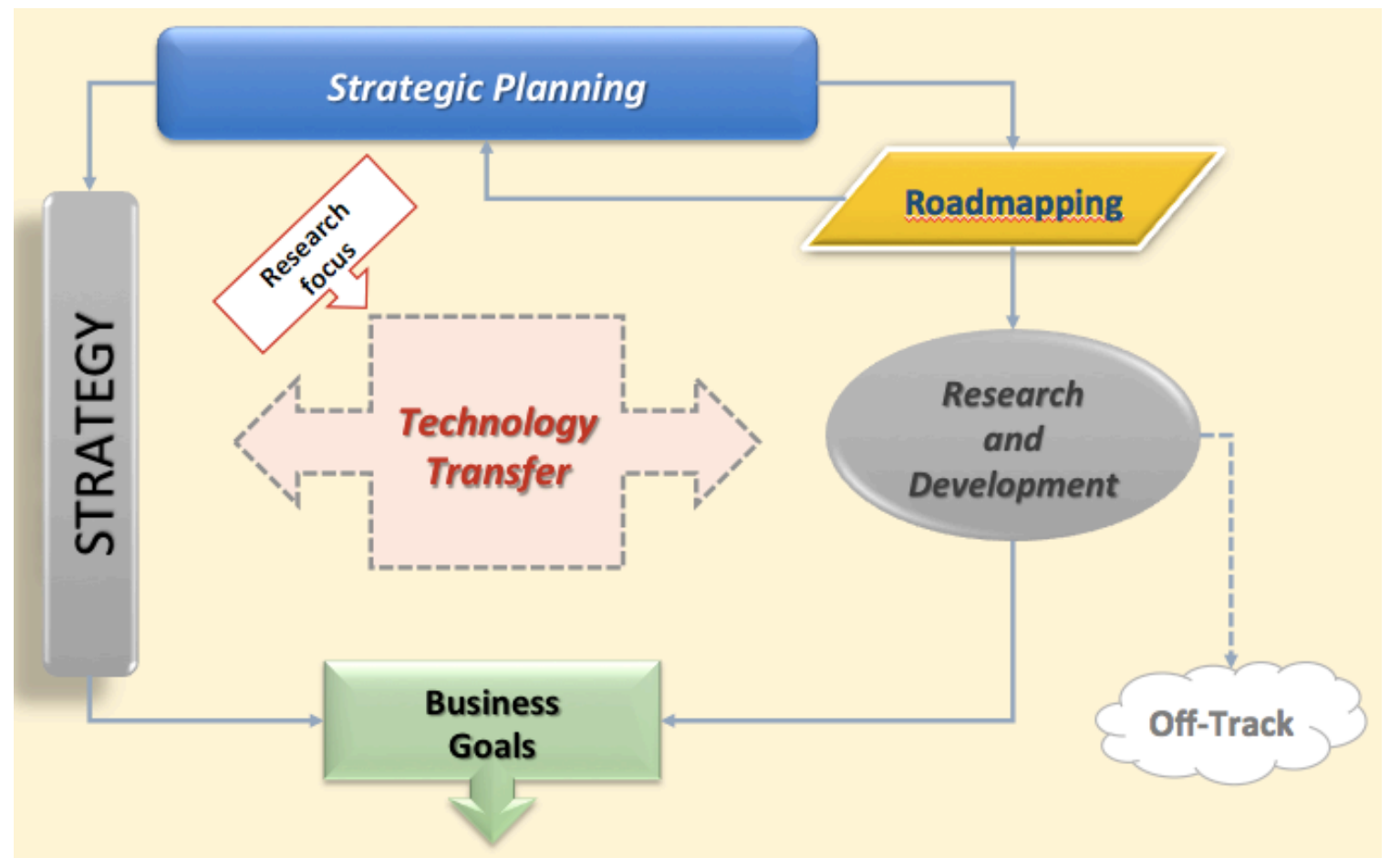

Figure 3 - TT as a Strategic Technology Control Process

Other aspects of the technology transfer process are also a subject of discussion in the literature. The evolution of a technology transfer process or the transformation the process can undergo in terms of organizational perception and awareness is one of those aspects. Mentioning previous relevant research in the field of knowledge management [154], Carayannis and Alexander describe this transformational process as a spiral-shaped one, and as vital to the success of R\&D collaborative projects., They further categorize the states of knowledge that organizations possess during the transformation: Ignorance of ignorance; awareness of ignorance; awareness of awareness, and ignorance of awareness [106]. The lifecycle management of an R\&D partnership, its dynamics, and its impact on the way 
technology is transferred in those partnerships is another aspect. Discussing government-university-industry strategic partnerships (GUISP), Carayannis et al. develop the idea that as those partnerships mature and evolve throughout time, and so should all managerial processes that support it, including technology transfer and knowledge sharing [155]. Since various developmental stages can be observed when studying GUISPs, each developmental stage with its own characteristics, it would be wise to try and adapt managerial processes to better fit those characteristics, in a way that is following the life cycle of the partnership, and that is more focused on efficacy. In the words of the authors, "since various GUISPs were established at different times, it is possible to compare them across different phases in their evolution while observing the entire lifecycle, in a 'snapshot-and-reel' approach" [155, p. 486]. Further mentioning the same concept, Carayannis et al. discuss the technology transfer process in universities in terms of steps, from the very basic research up to the creation of new products and generation of revenues. The steps are: Research and development, invention, disclosure, technology assessment, patent application, IP protection; marketing, business proposal; technology commercialization; new products and services; and revenue [33].

\section{Research Gaps}

Having understood the research landscape, and apprehended the relevant concepts, experiments, and frameworks available in the technology transfer literature and related fields', four major research gaps were identified as targets for this study, as follows: 
- There are not enough holistic and quantitative models to assess and measure an organization's TT capabilities, as the vast majority of studies are only concerned with the design of general technology transfer models and the identification of some important criteria and perspectives for the process [2][4], [7], [22], [34], [35], [38], [41]. An efficiency model has been developed, but it is exclusively focused on university technology transfer, and, the creation of a university technology transfer maturity scale and model [91]. Also, a quantitative multi-criteria decision-making model was applied to measure technology transfer potential, but the focus of the study was on research proposals and the front-end, not on the whole technology cycle, and not on organizational capabilities and process perspectives [19].

- There is a lack of studies on how to improve an organization's TT capabilities based upon its current status. As it was explained in the previous gap, most of the studies aim to identify aspects or criteria that are important for a technology transfer process. However, such studies are difficult to apply, and can be in contrast to what an organization already has in place. Literature has failed to assess the current status of an organization's TT process, and failed to provide improvement paths based on the current status making it harder for organizations to be more successful at their TT efforts [44].

- There is a lack of studies on internal TT overall. Studies mention the fact that technology transfer, as a whole, is still not very well understood and often produces inconsistent and unsuccessful results [3], [60]. Furthermore, within 
the technology transfer studies, the internal technology transfer type has been subject to only a few studies, to the point that some scholars are actively pushing for more research on this topic [9], [38], [144].

- There is a lack of studies on the ways that TT can help align R\&D and business strategy: Even when disregarding technology transfer, there is a lack of comprehension and research on how to align R\&D and technology development with business and corporate strategy [140]. In addition, most of the studies conducted up until now have been focusing on methodologies such as roadmapping [136], [140]-[142], [145]-[148] and other methods [137][139], [144]. None of these include technology transfer perspectives, which were already cited as important for the strategic control and alignment of technologies [1], [9], [97], [150].

In the next chapter, the above-mentioned gaps are put into perspective with the research goal and questions, and the methodology used is explained in more detail. 


\section{CHAPTER 3 - RESEARCH APPROACH AND METHODOLOGY}

\section{Research Gaps, Goals, and Questions}

Figure 4 illustrates the research gaps to be tackled, the research goal to be achieved, and the research questions to be answered.

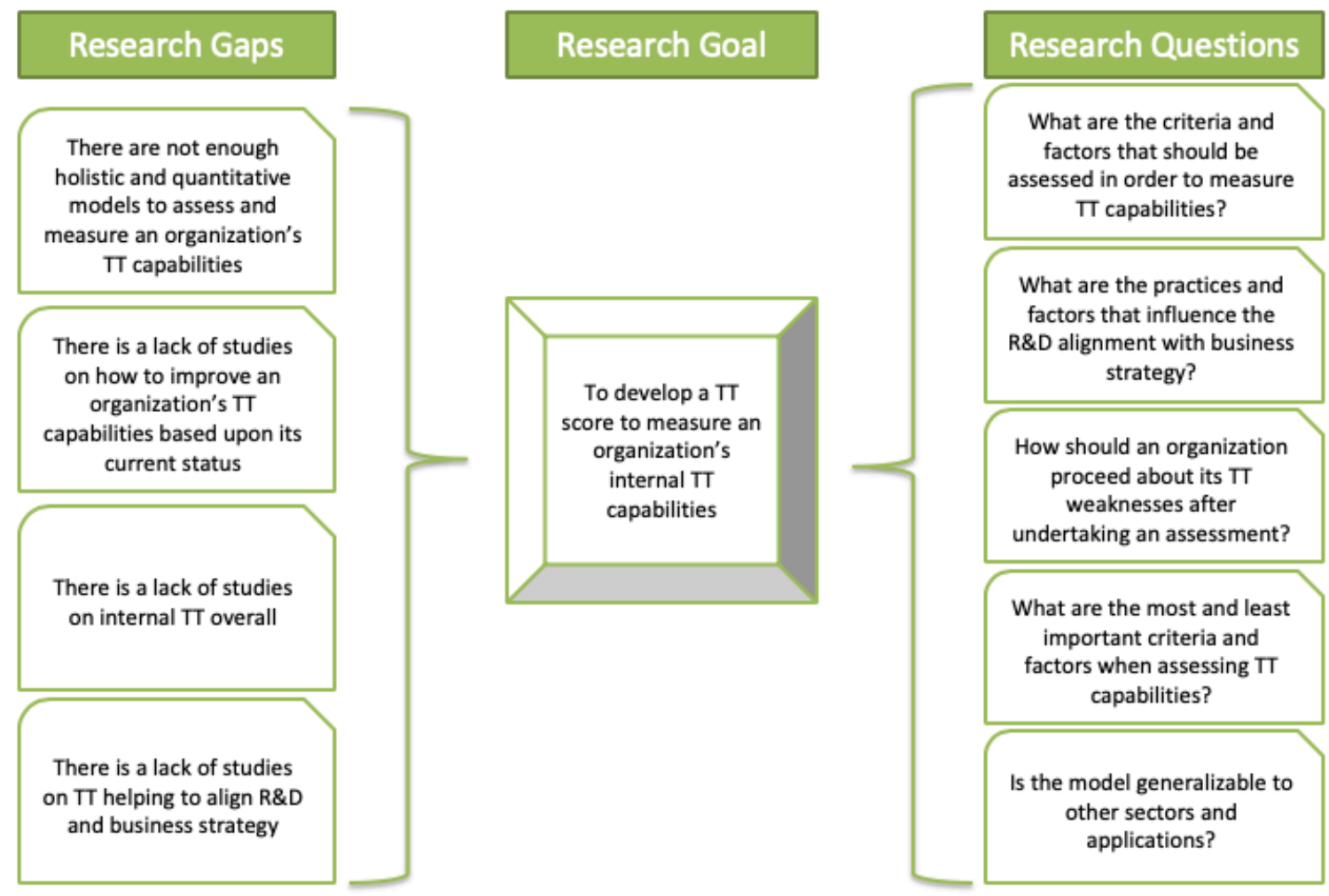

Figure 4 - Research Gaps, Goal and Questions

Inspired by, and leveraging a previous research by Estep [19], the goal of the proposed research is to develop a technology transfer score by means of building a holistic model to measure an organization's internal technology transfer capabilities. Such a score would allow the researcher to shed a light on the organization's TT weaknesses and strengths. From this point, it will also be possible to recommend actions to mitigate and/or eliminate these weaknesses. Also, by accounting for 
strategic planning factors, the model will enable a better alignment between R\&D, technology development, and corporate / business strategy. The organization will benefit both from the situational awareness about its TT process and from the possibility of improving the process.

The specific questions to be answered throughout this research are:

- What are the most and least important criteria and factors when assessing TT capabilities?

- What are the practices and factors that influence the R\&D alignment with business strategy?

- How should an organization proceed regarding its TT weaknesses after undertaking an assessment?

- What are the most and least important criteria and factors when assessing TT capabilities?

- Is the model generalizable to other sectors and applications?

\section{Research Methodology}

The methodology employed is a combination of action research in the first phase and a Hierarchical Decision Modeling in the second phase, as explained in the following sections.

\section{Action Research}

Action research (AR) is seen as a method for practitioners to get their hands

dirty and actively change something in the real-world, and it is also seen as an 
effective method to create knowledge [156]. Being a very diverse and dynamic methodology, authors argue that there is not a unique definition or manner in conducting action research [156]-[158]. O'Brien states that action research can be thought of as "learning by doing", and also lists several alternative names the methodology is referred to: Participatory research, collaborative inquiry, emancipatory research, action learning, and contextual action research [159]. Tripp defines it as any kind or variation of action inquiry, where the researcher aims to improve the practice by ways of acting upon it, and later inquiring on the action's results, in a cyclical fashion [160].

Action research brings together and leaps through the traditional divide between research and application since the methodology is incurred by researching with practitioners, and not researching on practitioners. It is a methodology that lies in the boundary between academia and practice [156]. In the words of Reason and Bradbury, "It seeks to bring together action and reflection, theory and practice, in participation with others, in the pursuit of practical solutions to issues of pressing concern to people, and more generally the flourishing of individual persons and their communities" [156, p. 1].

According to Reason and Bradbury [156], these are the four steps of action research:

- Step 1: Creating communities of inquiry within communities of practice, which creates. equality and collaboration between scientists and practitioners. 
- Step 2: Building theories in practice, which works with the practical sphere to build theory.

- Step 3: Combining interpretation with 'rigorous' testing. This tests the theory with practical applications.

- Step 4: Changing the status quo, and creating actual change to the practitioner's systems.

Similarly, Susman and Evered [161] describe the action research approach in five phases, which repeats in a cyclical manner:

- Phase 1: Diagnosing—identifying the problem.

- Phase 2: Action Planning—devising a plan on how to act on the problem.

- Phase 3: Action Taking-executing the plan.

- Phase 4: Evaluating-understanding the results of the actions taken.

- Phase 5: Learning-learning from the experience and starting the cycle over.

Action research is often referred to as a methodological approach rather than a method, i.e., several methods can fit inside this methodological approach. AR transforms reality as it is a methodological approach that has a performative perspective as one of its most important components [162]. Strengthening this view of action research as an approach rather than a method itself, Tripp argues that AR cannot be used as a single method in a dissertation. It will always require a second method to complement it [160]. Similarly, Dick emphasizes that AR, as an approach, 
takes advantage of several different methods and tools to achieve the desired changes [163].

Action research is a way through which researchers influence on or in a system, and this influence (action) also creates important knowledge about the system [161]. It is an approach that creates the conditions for better decision-making about practice since the process unfolds itself in a systematic way and within the practice [164]. To conduct AR, the researcher should, at the same time, actively engage in the action and reflect on the actions taken, generating positive changes to the practical system, and generating useful knowledge for the theory [165]. Action research is not only beneficial in practical aspects, but it also generates knowledge [163]. According to Chandler and Torbert, action research aims not only to understand a system, but also to present the future conditions of the system [157]. The approach is focused on resolving real issues, and is applied in real conditions and environments, rather than in enclosed, controlled, and experimental ones [159]. According to Ferrance, the action research approach brings benefits by focusing on the issue to be solved, and by allowing professional development of those involved [158].

Scholars also praise the iterative nature of AR. Tripp says that AR is an approach that makes use of different techniques to provoke changes in reality, and its iterative nature is possibly its most distinguishing feature. The end of a cycle is always the starting point of another, and serves as an improvement opportunity [160]. AR operates in iterative cycles of action and reflection on the action, bringing desirable changes that are not easily achieved otherwise [166]. 
The active participation of the researcher is a very important feature of AR. As Dick summarizes it, even when the word "participatory" is not used, the active participation of the researcher brings several benefits to the approach, such as the commitment to the actions that were agreed upon, the commitment to information sharing, and the commissioning of the people involved in the effort [163]. The participation of practitioners in the process is also important. In the words of Village et al., "in the AR approach, it is the responsibility of researchers and practitioners together to define the plan, carry out the initiatives and monitor what is helping or not helping achieve the goal in the organization" [165, p. 1576]. Furthermore, as Dick and Greenwood puts it:

"Action research rejects this pattern of behavior and organization. For action researchers a key concept is a dual commitment to both participants and action. Action research is done with rather than on, the participants - as is often stated. Ideally, the participants become equal partners and co-researchers. The research is done to provide learning and understanding (and theory) that can be used by participants to improve their situation for the benefit of all. For the most action researchers, as far as feasible these are imperatives" [166, p. 195].

While more popular and known in social sciences fields such as psychology, sociology and anthropology, action research is also indicated as a good way to tackle business and management issues. Although academics may use action research in a way that is excessively theory-oriented for business purposes, there is a balance to be reached so that consultants can make use of the approach to solve management problems [167]. According to Perona et al., action research would be useful for 
operations management research, particularly for modeling organizational processes [168]. The action research approach would enable a much deeper and detailed understanding of the organizations when compared to other traditional management research approaches, e.g. surveys and interviews, thus being more advantageous [169]. Specifically with regards to technology management, action research has also been recommended. In his research on knowledge management using participation action research, Otosson argues that, had he chosen other more traditional methods of research, the results he achieved would not have been possible [170]. The same author goes on to affirm that the approach has especially helped regarding gathering information and generating insights about new product development, innovation management, and change and project management [170].

As previously explained, action research does not belong to any particular realm or field. On the contrary, the fields of study that are explored using the action research approach are numerous and diverse. Table 18 brings a small sample of what can be found in the literature, regarding the application areas, subjects, and issues being undertaken through action research.

Table 18 - Action Research Application Areas

\begin{tabular}{|c|c|}
\hline Area / Subject & Reference \\
\hline Agricultural Production & {$[171],[172]$} \\
\hline Aviation & {$[167]$} \\
\hline Community Psychology & {$[173]$} \\
\hline Ergonomics & {$[165]$} \\
\hline
\end{tabular}




\begin{tabular}{|c|c|}
\hline Health Services & {$[174]-[177]$} \\
\hline Homelessness & {$[178]$} \\
\hline Industrial Design & {$[179]$} \\
\hline Manufacturing & {$[168]$} \\
\hline Immigration & {$[180]$} \\
\hline Procurement Management & {$[181]$} \\
\hline Religion & {$[182]$} \\
\hline Sexual Exploitation & {$[183]$} \\
\hline Supply Chain & {$[184]-[186]$} \\
\hline Teaching & {$[164]$} \\
\hline Technology Management & {$[169],[187]$} \\
\hline University - Industry Partnerships & {$[188]$} \\
\hline
\end{tabular}

As previously stated, AR is more of a research approach than a research method. It would frame the way the researcher regards the problem, the way the researcher interacts with the people involved in the problem and most importantly, AR would frame the way the researcher tries to solve the problem (research objective). In order to perform $\mathrm{AR}$, the researcher has to actively engage with practitioners, participating in discussions, and activities as a member of the team. Plus, after this active participation, changes have to be proposed and implemented, aiming to change the status quo, and improve the practitioners' systems. If the researcher only describes or facilitates discussions, and if the researcher does not produce any actual changes to the system, he/she will not have used action research. As an illustration of producing changes, which is a crucial aspect of action research, 
Table 19 summarizes the changes sought by researchers in some of the studies available in the literature.

Table 19 - Action Research Producing Changes

\begin{tabular}{|c|c|}
\hline Reference & Type of change produced in the system \\
\hline$[177]$ & Change patient education practices in a clinical diabetes setting \\
\hline$[172]$ & Change the innovation approach for sustainable agriculture in Europe \\
\hline [178] & Change of homeless women attitude through artwork \\
\hline [171] & Facilitate agricultural product standardization \\
\hline [189] & Implement good governance policies for healthcare technology management \\
\hline$[174]$ & Improve behavioral health services \\
\hline [187] & $\begin{array}{l}\text { Improve customer complaints management through an operations research multi } \\
\text { methodology }\end{array}$ \\
\hline [188] & $\begin{array}{l}\text { Improve knowledge-sharing partnerships between universities and small and } \\
\text { medium enterprises }\end{array}$ \\
\hline [167] & Improve sustainability in the aviation sector \\
\hline$[169]$ & $\begin{array}{c}\text { Improve technology management by creating a technology management assessment } \\
\text { framework }\end{array}$ \\
\hline [185] & Improve the process of supplier's evaluation \\
\hline$[175]$ & $\begin{array}{l}\text { Improve the experience of pregnant women in labor and their spouses when meeting } \\
\text { and dealing with their caregivers }\end{array}$ \\
\hline$[186]$ & Improving decision-making about whether to offshore manufacturing activities \\
\hline$[165]$ & Improving ergonomics in electronics manufacturing firm processes \\
\hline$[173]$ & Improving local urban regeneration \\
\hline$[181]$ & Improving purchasing processes in construction companies \\
\hline$[179]$ & Including human-centered design perspectives into software development \\
\hline$[182]$ & Overcome the culture of problem avoidance in non-profit organizations \\
\hline
\end{tabular}




\begin{tabular}{|c|c|}
\hline$[176]$ & Reducing conflicts and enhancing focus in surgery teams \\
\hline$[180]$ & Resolving identity issues in immigrant Pakistani women \\
\hline$[168]$ & Shorten manufacturing lead-time \\
\hline
\end{tabular}

As a research approach, AR can be combined with many different methods for data collection and data analysis. For instance, one could use AR as an approach, use focus groups or interviews as data collection methods, and use statistical analysis or grounded theory data analysis methods. In truth, action research must be combined with data collection and data analysis methods, because it is not a method in itself, but a methodological approach (as explained above). As explained earlier, action research requires a second method to complement it [160]. That assertion is proven by analyzing the literature with an exception made to the conceptual papers that discuss action research as an approach, rather than applying it. A myriad of different methods of data collection and data analysis can be effectively and successfully blended into action research, as shown in Table 20.

Table 20 - Action Research and Auxiliary Methods

\begin{tabular}{|c|c|}
\hline Reference & Auxiliary Method \\
\hline$[181],[185],[186]$ & Analytical Hierarchy Process (AHP) \\
\hline$[160],[165],[167],[169],[171],[176],[181]$, & Case Study \\
\hline$[185],[186]$ & Delphi \\
\hline$[162]$ & Focus Groups \\
\hline$[174],[185]$ & \\
\hline
\end{tabular}




\begin{tabular}{|c|c|}
\hline$[175]$ & Inductive Interpretative Qualitative Data \\
& Analysis \\
\hline$[167],[175]-[177],[182],[187]$ & Interviews \\
\hline$[162]$ & Social Network Analysis (SNA) \\
\hline$[176]$ & Statistical Analysis \\
\hline$[184]$ & Surveys \\
\hline$[177],[187]$ & Workshops \\
\hline
\end{tabular}

It is clear that AR is a good fit to work with qualitative and quantitative methods that use experts' opinions. Moreover, it has been argued that action research would work well with methods that use ranking and pairwise comparisons, and it should be more utilized coupled with quantitative methods [162].

Previous research has been done coupling these two methodologies, most importantly in the supply-chain management field. In the work of Arantes et al., the authors used AR and AHP to inform a construction company's purchasing strategy, which took place over the course of six months. Meetings were conducted between teams of researchers and practitioners to understand the situation, and to come up with the important criteria, sub criteria, and alternatives for the model [181]. The alternatives were construction items, and the results of the model application informed the company on its bottlenecks regarding items to purchase, ultimately feeding them with information of how to improve their purchasing strategy [181]. In the work of Dey et al., the authors use a combination of AHP and QFD (Quality Function Deployment) to analyze the performance of suppliers in a manufacturing company in the UK (United Kingdom) [185]. The authors state that focus groups were 
used throughout the development and application of the model [185]. In the work of Schoenherr et al., an AHP model was put together to work with a supply chain management problem in order to select the best offshore sourcing option for an American manufacturing company.[186]. The authors argued the need to use AR in the beginning of the project to understand the company situation, and also throughout the project, especially when implementing the best strategy identified by the model.

Analyzing these previous works with similar methodological approaches, it is important to summarize their main limitations and differences compared to the approach used in this research. The action research component of these articles was comprised of meetings in which they discussed possible criteria for the models, and the present study makes use of past experience in TT projects to inform the model development, supplementing items found in the literature. Previous works used AHP instead of HDM; they did not mention looking at the literature for important criteria-apparently the practitioners dictated all model components; there was no model validation process; only internal experts were used in the model applicationno external sources were used to validate or quantify the model, leading to the creation of unique, non-generalizable, and somewhat biased models; the model was used to rank alternatives, unlike the present study's approach of assessing an organization through a score; and no inconsistency, disagreement, or sensitivity analyses were conducted.

To further the understanding of AR and its applicability in this research, I proceed now to present and analyze other methods that could be seen as 
complements or substitutes to action research, namely case study, interviews, participant observation, and focus groups.

\section{Similar Methods}

\section{Case Study}

A case study is a research method that deeply analyzes one specific situation (the case) without separating it from its context [190]. As explained by Ellet, a case study is a form of analysis that can be applied to several levels, be it a whole region or country, or be it on a smaller level, such as a single organization [191]. Yin argues that case studies are ideal for situations where the context that involves the subject is crucial to its understanding. For example, an experiment would mimic the real situation but would do so in such a way as to completely isolate the subject from its context [190]. In the words of the author, "in brief, a case study allows investigators to focus on a case and retain a holistic and real-world perspective - such as in studying individual life cycles, small group behavior, organizational and managerial processes, neighborhood change, school performance, international relations and the maturation of industries" [190, p. 4]. Case studies are seen as a testing method, and are cited as especially useful in testing theories from the management sciences [192]. Moreover, case studies are indicated as a good way of applying and testing models to check their performance [193]. As opposed to some other popular methods, e.g. participant observation and interviews, case studies are not mere data collection methods [190]. The method is very popular in business studies [192], and it is usually applied to analyze and make inferences about one specific case, thus avoiding one of 
its biggest limitations, which is the lack of the ability to confidently generalize results to other cases [190].

Although business studies are the main area where cases are used, several different application areas have explored the method, such as patent analysis [193], policy-making for technology transfer [194], e-government technology transfer [195], population safety strategies in the event of crises / tragedies [196], renewable energy technology commercialization [114], risk assessment for hazard installations [197], and road pavement maintenance [198], among others.

The objectives used in a case study, as opposed to those used in action research, are not to necessarily to cause changes to a system or situation, but mostly to test a model, demonstrate an idea, or understand a certain setting. Table 21 depicts a summary of different objectives achieved by means of using case study.

Table 21 - Case Study Objectives

\begin{tabular}{|c|c|c|}
\hline Reference & Objective & Description \\
\hline$[193]$ & Apply a Model & Apply a technology transfer model \\
\hline$[199]$ & Apply a Model & Apply a risk assessment model \\
\hline$[198]$ & Compare Quantitative & Compare AHP versus TOPSIS for prioritization \\
& Methods & purposes. \\
\hline$[196]$ & Demonstrate a Model & Test scenarios to rank technology transfer strategies. \\
\hline$[194]$ & Test Different Scenarios & Review and test relationships between constructs \\
\hline$[114]$ & Test Relationships & form a theoretical framework. \\
& & Understanding e-government technology transfer \\
\hline$[195]$ & Understanding and & \\
& Describing an Issue & \\
\hline
\end{tabular}




\section{Interviews}

Interviewing is a data collection method [200], which means that researchers have to make use of other methods for posterior data analyses. Interviewing is a way through which researchers engage in scientific inquiry with the participants [201]. Interviewing is a good method to access information about how people perceive things [202]. In the words of Weiss:

"We can learn also, through interviewing, about people's interior experiences. We can learn what people perceived and how they interpreted their perceptions. We can learn how events affected their thoughts and feelings. We can learn the meanings to them of their relationships, their families, their work and their selves. We can learn about all experiences, from joy through grief, that together constitute the human condition" [202, p. 1].

Interviews have been used as a solid data collection method for decades, but it was not until the 1950s that it became a mainstream method. Until the late 1950s, participant observation was seen as the best way to get data from people, but from then on, interviews started to appear as an alternative, and are especially attractive if the researcher objective is to capture a person's subjective understanding of something [201]. As explained by Weiss, there are two types of interviews: quantitative (survey interviews) and qualitative (qualitative interviews). Survey interviews have an advantage of being very precise by gathering exactly the same information of every respondent by asking the same questions, and by allowing only a few categories as possible answers. In addition, it is easier to work with large samples, and to also produce comparative reports between groups and descriptive 
and predictive statistical analyses. On the other hand, by using qualitative interviews, the depth and quality of information is greater, details are better apprehended, and the outcomes (reports and interpretations) are able to bring a much better and deeper understanding of the respondents' feelings and experiences about the subject / event being studied [202]. In a social sciences environment, Seidman created a three-interview series method for conducting in-depth interviews [201]. It consists of interviewing each person three times, for 90 minutes each time, and having an interval of three days to a week between each interview. The questions would be open-ended, and the interview would be conducted in an unstructured or semistructured way. The first interview is focused on delineating the person's experience about the studied subject. The second interview seeks to capture as much details as possible about the experience. The third and last interview seeks to understand the meaning of that experience to the interviewee.

There are several objectives that can be achieved by means of applying interviews, as it will be shown later in this study. As listed by Weiss, developing detailed descriptions, integrating multiple perspectives, describing process, developing holistic description, learning how events are interpreted, bridging intersubjectivities, identifying variables and framing hypotheses for quantitative research are among them [202]. The purpose of interviewing is to comprehend a person's experience about something and also to comprehend the meaning of that experience [201]. The most mentioned downside of the method is that it can be very labor-intensive and time-consuming [201], [202]. 
Regarding the application areas, interviews are applicable to virtually any situation in which the researcher wants to capture the participants' perceptions. Applications areas can be, but not limited to, mental health studies [200], motivational interviewing [203]-[208], technology innovation [209], and disease prevention [210].

As it happens with case studies, the objectives of conducting interviews are not to change the practitioner's system, but rather to get a sense of what the study participants perceive regarding a particular issue, setting, or situation. Table 22 summarizes the objectives of some studies that have used interviews as a data collection method.

Table 22 - Interview Objectives

\begin{tabular}{|c|c|c|}
\hline Reference & Objective & Description \\
\hline [207] & $\begin{array}{c}\text { Comparing different approaches } \\
\text { of motivational interviewing }\end{array}$ & $\begin{array}{c}\text { In a healthcare setting, the effectiveness of } \\
\text { numerous motivational interviewing strategies } \\
\text { was compared. }\end{array}$ \\
\hline [203] & $\begin{array}{l}\text { Deeply understanding one's } \\
\text { problems }\end{array}$ & $\begin{array}{l}\text { Understand and help to treat people's disease } \\
\text { and lifestyle problems }\end{array}$ \\
\hline [208] & $\begin{array}{l}\text { Evaluation of different training } \\
\text { approaches }\end{array}$ & $\begin{array}{c}\text { In a behavioral health setting, the effectiveness of } \\
\text { distance training was evaluated }\end{array}$ \\
\hline [210] & $\begin{array}{l}\text { Examining different forms of } \\
\text { disease intervention } \\
\text { adaptations. }\end{array}$ & $\begin{array}{l}\text { Understanding and evaluating the effectiveness } \\
\text { of adaptations to HIV treatments. }\end{array}$ \\
\hline [204] & $\begin{array}{l}\text { Measuring skills in motivational } \\
\text { interviewing }\end{array}$ & $\begin{array}{l}\text { Clinicians were evaluated concerning their } \\
\text { motivational interviewing skills. }\end{array}$ \\
\hline
\end{tabular}




\begin{tabular}{|c|c|c|}
\hline [205] & $\begin{array}{c}\text { Measuring motivational } \\
\text { interviewing methods transfer }\end{array}$ & $\begin{array}{c}\text { Pilot testing a training model to transfer } \\
\text { motivational interviewing methods. }\end{array}$ \\
\hline$[206]$ & $\begin{array}{c}\text { Measuring variability in } \\
\text { motivational interviewing }\end{array}$ & $\begin{array}{c}\text { Port-training follow-up to check variability in } \\
\text { motivational interviewing skills. }\end{array}$ \\
\hline$[209]$ & $\begin{array}{c}\text { Understanding research and } \\
\text { development practices }\end{array}$ & Understand how Chinese enterprises conduct \\
& & R\&D \\
\hline
\end{tabular}

\section{Participant Observation}

Participant observation is another data collection method, as evidenced by Chaudhry et al. [211]. A participant observer watches the other participants in order to understand how they go about a situation. This is very popular in ethnographic studies where the ethnographer uses participant observation to understand and describe cultural phenomena and cultural behaviors [212]. The process of observing participants in a real setting involves the registration, interpretation, and recording of observations. The type of data it yields is circumscribed by the influence the observer exerts over the observed and vice versa [213]. Although observing and taking notes would suffice in some cases, video-taping is recommended for better quality observations [214]. In 1955, Schwartz and Schwartz divided the participant observer in two types: active and passive. The active observer would have close contact with the participant, trying to blend in to gather more detailed information. "His intention is to experience the life of the observed so that he can better observe and understand it" [213, p. 349]. The passive observer would try to stay as separate as possible from the participant, as he/she sees contact as an undesirable 
interference. "The investigator assumes that the more passive he is the less will he affect the situation and the greater will be his opportunity to observe events as they develop [213, p. 348]. More recent works have created different levels of interaction between observer and observed. According to Spradley, there are five levels of interaction: Nonparticipation-the researcher is completely isolated/hidden and does not engage with other participants; passive participation-the researcher is in the scene, and acts as a "by-stander", but does not engage with other participants; moderate participation-the researcher is in the scene but alternates between the passive and active types of observation; active participation-the researcher participates and does whatever the other participants do, observing ordinary participants from inside the situation; complete participation-this type occurs when the researcher is already an ordinary participant and decides to research that situation [212]. Even when the researcher has close contact with participants, there are several important distinctions between the two. In his 1980 book, James Spradley lists six of the most important differences: Dual purpose, explicit awareness, wideangle lens, introspection, record keeping [212], as explained below.

- Dual purpose: The participant observer can participate in the activities, but also observes the other participants attentively.

- Explicit awareness: While ordinary participants do not care or pay attention to the details of the environment and people around them, the participant observer watches and registers as many details as possible about the 
situation and the way people go about it, e.g., the way people talk, walk, sit, look, etc...

- Wide-angle lens: A participant observer pays attention not only to "important" details of a situation, but also pays attention to "trivial" details. A participant observer is explicitly paying attention and pays attention to a wide variety of trivial situations and behaviors.

- Introspection: Often times participant observers will participate in the activities they are observing and reflect on their own feelings and impressions about it even down to the smaller details. Conversely, ordinary participants do most of their actions "automatically" without giving any thought about it.

- Record keeping: The participant observer will meticulously register every detail and impression he/she has about the people and situations he/she is observing.

Most of the studies that use participant observation as a data collection method are situated in the social sciences or health sciences realm. However, in some cases, researchers in technology management and business also make use of the method. As a sample of application areas, there have been studies on childrens' behavior [215], critical care nursing [216], economics [211], psychiatric nursing [214], tourism [217], R\&D management [218], and university technology transfer $[22]$. 
In general, the objectives achieved by means of deploying participant observation as a data collection method are to describe a situation or analyze a participant's behavior in a certain setting. Again, at least by itself, to change a system would not be an achievable objective by applying participant observation. Table 23 summarizes some of the studies found on the literature using participant observation, along with their objectives.

Table 23 - Participant Observation Objectives

\begin{tabular}{|c|c|c|}
\hline Reference & Objective & Description \\
\hline$[22]$ & Behavior Analysis & Analyzing behavioral aspects in a technology transfer \\
& & context. \\
\hline$[218]$ & Behavior Analysis & Analyzing and comparing conventional versus highly \\
& autonomous small team-type R\&D management. \\
\hline$[214]$ & Describing a Method & Describe videotaped recording as a data collection \\
& & \\
\hline$[211]$ & Behavior Analysis & Perform non-market economic valuation in \\
& & \\
\hline$[216]$ & Understanding the & Identification of the meaning of a technology \\
& Usefulness of a Technology & (mechanical ventilation) for critical care nurses. \\
\hline$[217]$ & Behavior Analysis & Understanding senior tourists' behavior \\
\hline$[215]$ & Behavior Analysis & Understanding the process of friendship making \\
& & \\
\hline
\end{tabular}




\section{Focus Groups}

One data collection method used to obtain information is focus groups [219][222][223]. This method can be used to gather data from three distinct levels of analysis: The participant, the group of participants, and the interaction between and among them [220]. As explained by Greenbaum in his 1998 book, focus groups can be categorized into three groups: Full groups, mini-groups, and telephone groups, as explained below [223]:

- Full groups: 8 to 10 persons in a session directed by a trained moderator for about 90 to 120 minutes. Participants are chosen based on common characteristics, such as demographics or market interests.

- Mini-groups: 4 to 6 persons in a session directed by a trained moderator for about 90 to 120 minutes. Participants are chosen based on common characteristics, such as demographics or market interests.

- Telephone groups: Participants engage in a teleconference directed by a trained moderator for about 30 to 120 minutes. They are chosen based on common characteristics, such as demographics or market interests.

Notwithstanding its popularity as a method, successful focus groups are not an elementary or easy activity. Some of the critical characteristics of a successful focus group effort are: Authority of the moderator; using both verbal and non-verbal inputs; benefitting from the group dynamics in the room; and achieving concentrated attention among the participants; among others [224]. The moderator, a very important actor in the focus group research, is very different from an interviewer and 
should drive the discussion with as little interference as possible. As opposed to what happens in an interview, the moderator does not ask questions and interact as few times as possible with the participants. Following a pre-established guide, his/her main role is to make sure that the participants are speaking their minds, giving their opinions, and discussing the subjects that are important to the research[223].

The activities and discussions conducted when applying focus groups can vary significantly, and each researcher should be keen enough to choose the most proper types of activity in order to achieve his/her objectives. Colucci lists nine types of activities/techniques that can drive discussions in focus groups [219].

- Choosing alternatives: Each participant chooses a subject from a pool of alternatives and discusses them.

- Free listing: Participants make lists of subjects or sub-topics in order to start and drive discussions.

- Label generation: Participants use sticky notes (labels) to note important aspects about subjects, and discuss them until all aspects are covered.

- Magic tools and fantasy: Magic stories and fantasy tales can be used to drive participants to imagine, create, and discuss solutions for an issue.

- Picture sorting: Participants sort pictures from a pool to match specific characteristics of a topic, and they discuss why and how those pictures are a good fit to describe that situation.

- Pile sorting: Cards, papers, or objects can be sorted in piles with the aim to denote similarities or differences between subjects. 
- Ranking: Participants rank elements in a certain order to represent a situation or issues, and a discussion ensues.

- Rating: Subjects are listed, and participants rate them in order to filter items out, which then focuses the discussion.

- Storytelling: Participants are prompted to create and tell stories representing certain situations in order to start the discussion, and to look for solutions for an issue.

The intense interaction between participants represents the strength of focus groups as a data collection method. An advantage of focus groups is that they lead participants to discuss and react to each other's opinions, thus creating a deeper and wider understanding of their reflections on the subject being studied [224]. In the words of Greenwood et al., "Focus groups are an effective mechanism to generate understanding and gain insight into the research participants' world" [225, p. 90].

Focus groups can be seen as an alternative to traditional interviewing when the researcher is concerned about the accuracy of the latter method [226]. Several studies have also been conducted merging focus groups and AHP [203], [227]-[229]. It is important to note, however, that none of those studies have performed action research, or validated the model, having used focus group to discuss the components and results of the application of an AHP model.

Regarding the usage of focus groups, it has been heavily adopted by companies and consultancy groups to develop market research and other marketing-related 
studies [219]. Thomas Greenbaum lists nine applications of focus groups that could benefit companies and marketing professionals: New product development studies, positioning studies, habits and usage studies, packaging assessments, attitude studies, advertising evaluations, promotion evaluations, idea generation, motivation studies [223]. Application areas for focus groups are, among others, agriculture [227], airline service [230], construction projects [228], [231], dementia care [221], diabetes [232], real estate industry management [229], technology transfer [222], [233], [234], suicidal behavior [219].

As for the objectives of studies that make use of focus groups, the vast majority aims to identify trends and characteristics of a subject, or to better understand that subject. As it happens with all other data collection methods discussed so far, none of them has the impetus to change the system being studied, as opposed to what action research does. Table 24 summarizes the objectives of some of the studies found in the literature, all of which use focus groups as a data collection method.

Table 24 - Focus Groups Objectives

\begin{tabular}{|c|c|c|}
\hline Reference & Objective & Description \\
\hline$[227]$ & Criteria Selection for an & Selecting strategies for rice stem borer management \\
& AHP Model & \\
\hline$[230]$ & Criteria Selection for an & Competitive service quality benchmarking in airline \\
& AHP Model & industry \\
\hline$[228]$ & Discuss AHP Model & Risk identification and assessment of modular \\
& Results & construction \\
\hline
\end{tabular}




\begin{tabular}{|c|c|c|}
\hline [233] & $\begin{array}{c}\text { Identification of } \\
\text { Research Questions }\end{array}$ & $\begin{array}{l}\text { Discussion of perspectives of innovation and technology } \\
\text { transfer }\end{array}$ \\
\hline [229] & $\begin{array}{l}\text { Identification of Subject } \\
\text { Factors }\end{array}$ & $\begin{array}{c}\text { Identification and discussion of TQM constructs in } \\
\text { organizations }\end{array}$ \\
\hline [234] & $\begin{array}{l}\text { Identification of Subject } \\
\text { Factors }\end{array}$ & $\begin{array}{l}\text { Identification and ranking of supporting factors for IT- } \\
\text { supported international technology transfer. }\end{array}$ \\
\hline [221] & $\begin{array}{l}\text { Identification of Subject } \\
\text { Factors }\end{array}$ & $\begin{array}{l}\text { Identification of barriers and facilitators in dementia } \\
\text { care. }\end{array}$ \\
\hline [222] & $\begin{array}{c}\text { Understanding of a } \\
\text { Process }\end{array}$ & Identifying phases of technology transfer. \\
\hline [219] & $\begin{array}{l}\text { Understanding of an } \\
\text { Issue }\end{array}$ & $\begin{array}{c}\text { Understanding why young people in Universities commit } \\
\text { suicide. }\end{array}$ \\
\hline [232] & $\begin{array}{l}\text { Understanding of an } \\
\text { Issue }\end{array}$ & $\begin{array}{c}\text { Understanding Canadian indigenous people's } \\
\text { experiences in managing their diabetes problems. }\end{array}$ \\
\hline [231] & $\begin{array}{l}\text { Understanding of an } \\
\text { Issue }\end{array}$ & $\begin{array}{l}\text { Understanding the effects of Building Information } \\
\text { Modeling (BIM) on collaborative design. }\end{array}$ \\
\hline
\end{tabular}

\section{Why Action Research?}

Looking at Tables 21 through 24, the difference between the methods is clear. While action research heavily focuses on producing changes in an organization's systems, the other methods focus on identifying aspects, trends, constructs, describing actors and situations, or understanding issues. At least in this, changeoriented nature of action research represents a benefit when developing a hierarchical model. Since technology transfer is a very complex matter, a model that 
aims to capture the important aspects of a TT process and to measure an organization TT capabilities would benefit from a research approach that takes a deep dive in the day to day activities of a TT team, and would also benefit from a research approach that takes into account what was learned from previous changes in the practitioners' systems. Furthermore, action research has the best of two worlds as it joins the dimensions of observation and participation in a single approach. Focus groups, even when utilized to find criteria for a model, are not as cohesive as AR. Focus groups lack the component of provoking actual changes in the practitioners' systems, therefore they only captures the perceptions and thoughts of practitioners, and not what they actually do/did on a daily basis, nor what they try/tried to implement to improve their processes. Action research goes beyond the mere discussion of ideas and solutions because it discusses the situations, proposes changes, implements the changes, and analyzes the results, thus being a deeper, more accurate, and trustworthy approach, if one is to measure and recommend changes in managerial processes. Interviews, as much as they capture the perceptions of participants about a certain subject, also fall short when balancing out these perceptions with day-today actions. As much as participant observation would be able to tell what practitioners do on real settings and not only what they think about something, it is important to capture both dimensions, what people really do, and what they think about it. Action research has the ability of capturing both dimensions. It is a tremendous approach for discussing issues with practitioners, thus capturing their ideas and perceptions, and it is also very good for observing and working with practitioners in a real setting, thus enabling the researcher to contrast those 
perceptions against what practitioners really do, thereby accounting for their challenges and everyday issues. Moreover, AR provides a perfect "finishing touch" when it implements novelties in a system and analyzes those changes, then starts the cycle over. These characteristics make action research a good aid in the development of the model, which is composed of items coming from the literature (knowledge accumulated by decades of other studies), and also items coming from a practical experience that takes into account observations of practitioners, their perceptions and ideas, and also their behavior and real actions and challenges.. Lastly, consistent with what was presented and discussed in the research gaps section, current technology transfer literature does not demonstrate studies capturing the entirety of relevant factors involved in technology transfer, hence the need for an additional source of information (coming from practitioners through the action research piece) when building a model such as the one in this study.

Nevertheless, as evidenced by the information brought by the literature review, it would be unfair to directly and fully compare action research with the other methods discussed here, namely interviews, participant observation, and focus groups since those are data collection methods, whereas action research is a research approach. In an analogy using organizational structure, action research would sit at the strategic level, while the other methods would be placed in the tactical and operational levels. Moreover, AR being a research approach rather than a method, it can be easily applied in conjunction with other methods, including the data collection methods discussed here, thus joining forces and producing better results. 
Compared to the traditional way of developing HDM models, the proposed approach is more of an addition rather than a drastic change. All of the components of a traditional HDM deployment are still present, namely literature review, consultation with experts, model validation and so on, hence there is no methodological risk. The novelty here is to take advantage of the action research approach to enrich the model, providing it with more accuracy and fidelity to the reality of practitioners, and their needs and challenges.

Compared to the other methods analyzed and discussed in this research, the case study method is a perfect complement to the "literature-AR mixed HDM model". A case study is not a method to collect data, such as interviews, focus groups, and participant observation, but it is a form of analysis that can be very useful to test or implement a model. Furthermore, as previously mentioned, action research cannot be used in a dissertation as the sole method of analysis [160]. Using Bonneville Power Administration's TI Program as a case study to test the model seems to be a solid and reasonable approach. Case studies are also a good match within an action research cycle, e.g., one could discuss and propose changes in the system (with the participation of practitioners), and then implement and analyze the changes as a case study. Nevertheless, if the proposed changes did not involve active work and discussions with practitioners (changes based only on the literature, for example), the implementation would be a case study, but the approach would not be action research.

Finally, in addition to serving as an enhancement of the data collection for the model, there is another advantage of using action research as an aid for HDM.. By 
conducting an action research project, the researcher is closely involved with practitioners, gaining their confidence, and getting to know the organization and the environment better. This presents the researcher with a better position to apply the model, and to draw more accurate conclusions and recommendations unlike using a more distant and isolated approach, such as consulting the literature and rapidly consulting with experts to validate the model, using quick interviews, or focus groups / participant observation sessions.

In summary, action research was used as a methodological approach in the preliminary stages of the research to help build the initial model, and HDM was used for the remaining stages. Figure 5 summarizes the methodological approach being proposed. 


\section{Action Research and HDM Integration}

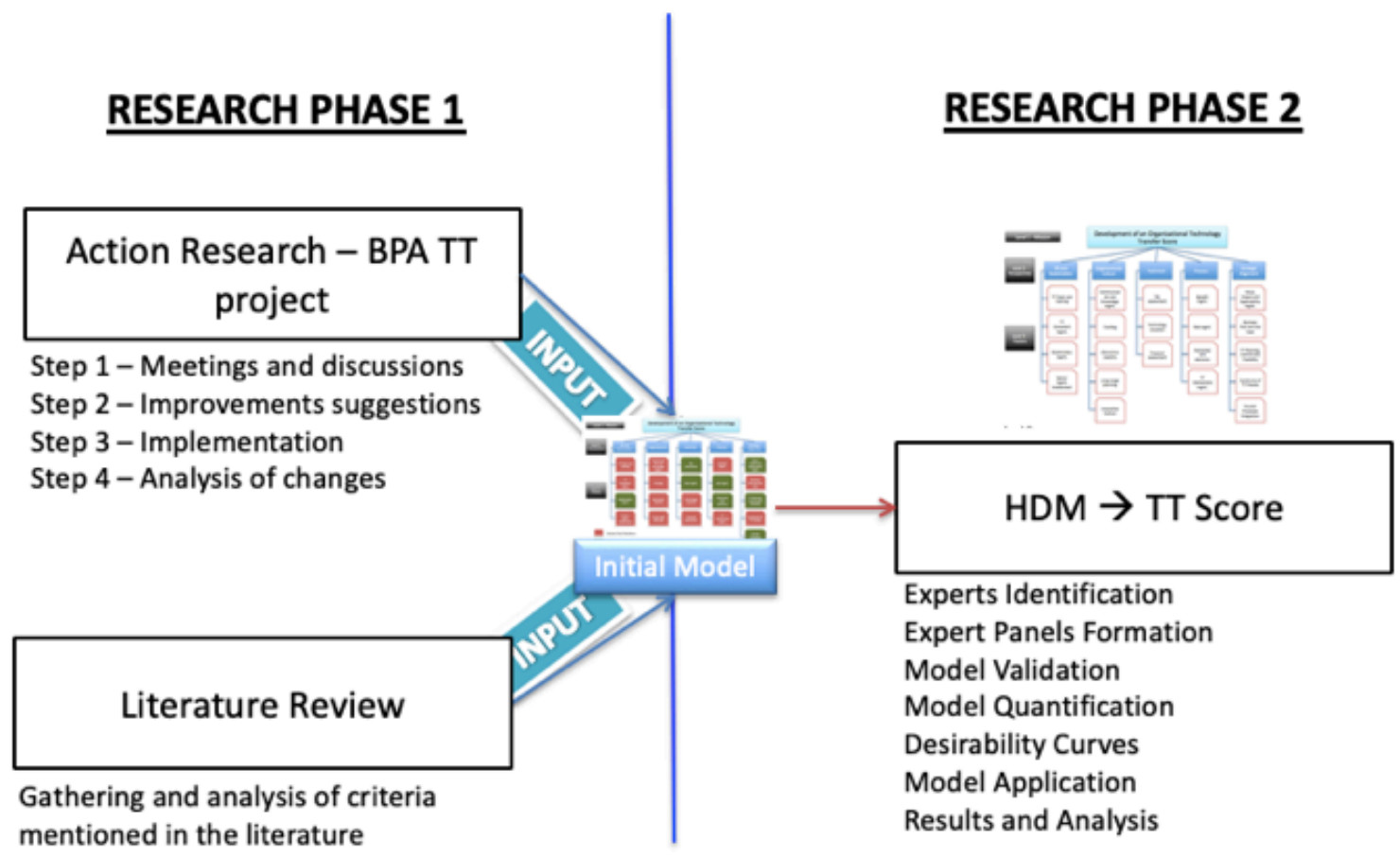

Figure 5 - Methodological Approach - AR and HDM Integration

As shown in Figure 5, in the Research Phase 1, the four basic steps of action research were conducted at Bonneville Power Administration (BPA). In the transition from Research Phase 1 to Research Phase 2, the experience and knowledge acquired during the AR project, along with the data from the literature, was used as an aid to develop the initial model. As further explained in the next section, Phase 2 brings in the HDM component.

\section{Hierarchical Decision Modeling (HDM)}

Hierarchical Decision Modeling (HDM) is a Multi-Criteria Decision Making (MCDM) method that was developed in the 1980s by Kocaoglu [235]. The basic idea of HDM is to represent the problem in a hierarchical format so that the decision 
makers can visualize which items (criteria and sub-criteria) can affect the objective/mission. According to Munkongsujarit et al., HDM helps the decision maker by presenting the decision problem as a cascade of problems that are simpler to handle [236]. This model breaks down the various elements of the problem into smaller sub-problems such that the decision problem is represented as a hierarchy [237]. HDM is a tool used in decision making to rank and evaluate the available alternatives and to determine the best among them [236]. It is a tool that helps decision makers quantify and incorporate quantitative and qualitative judgments into a complex problem [238].

HDM has been used in a variety of cases and for several purposes, especially in technology management, to evaluate and understand which technology alternative is the best option in a particular setting, given the criteria established to evaluate the alternatives. According to Munkongsujarit et al., hierarchical decision models assist the decision makers by providing a systematic way to evaluate all available alternative solutions to the problem according to the relative importance of the criteria, and then to identify the best possible solution [236].

The basic structure of HDM can vary depending on the needs of each application. The most traditional structure contains five levels: : Mission, Objectives, Goals, Strategies, and Actions (MOGSA), as shown in Figure 6.However, simpler structures can be used, such as a three-level model containing Mission, Criteria, and Alternatives (Figure 7) or a four-level model containing Mission, Criteria, SubCriteria, and Alternatives. According to Sheikh et al., with HDM, multiple perspectives 
can be prioritized and their associated criteria can be ranked [239], highlighting which criteria and/or perspectives are more important and to what degree.

In order to apply HDM, it is necessary to select experts from within the specific studied field, who will help create the model and evaluate the relationships between objective, criteria, sub-criteria, and alternatives. The experts make pair-wise comparisons among the items (criteria, sub-criteria, and alternatives) in the model to determine the weights and relationships using the constant-sum method, which divides a total of 100 points between the items that are being evaluated. The results of the comparisons are then extracted into matrixes, which in turn will normalize the values, and process them in order to rank the alternatives. In the end, it is possible to determine which alternative is the best by taking into consideration the criteria and evaluations made by the experts involved. As Turan et al. state, in the HDM model, pair wise comparisons are made to express the importance of one element of the decision problem, the criteria, with respect to another, the alternatives [240]. 


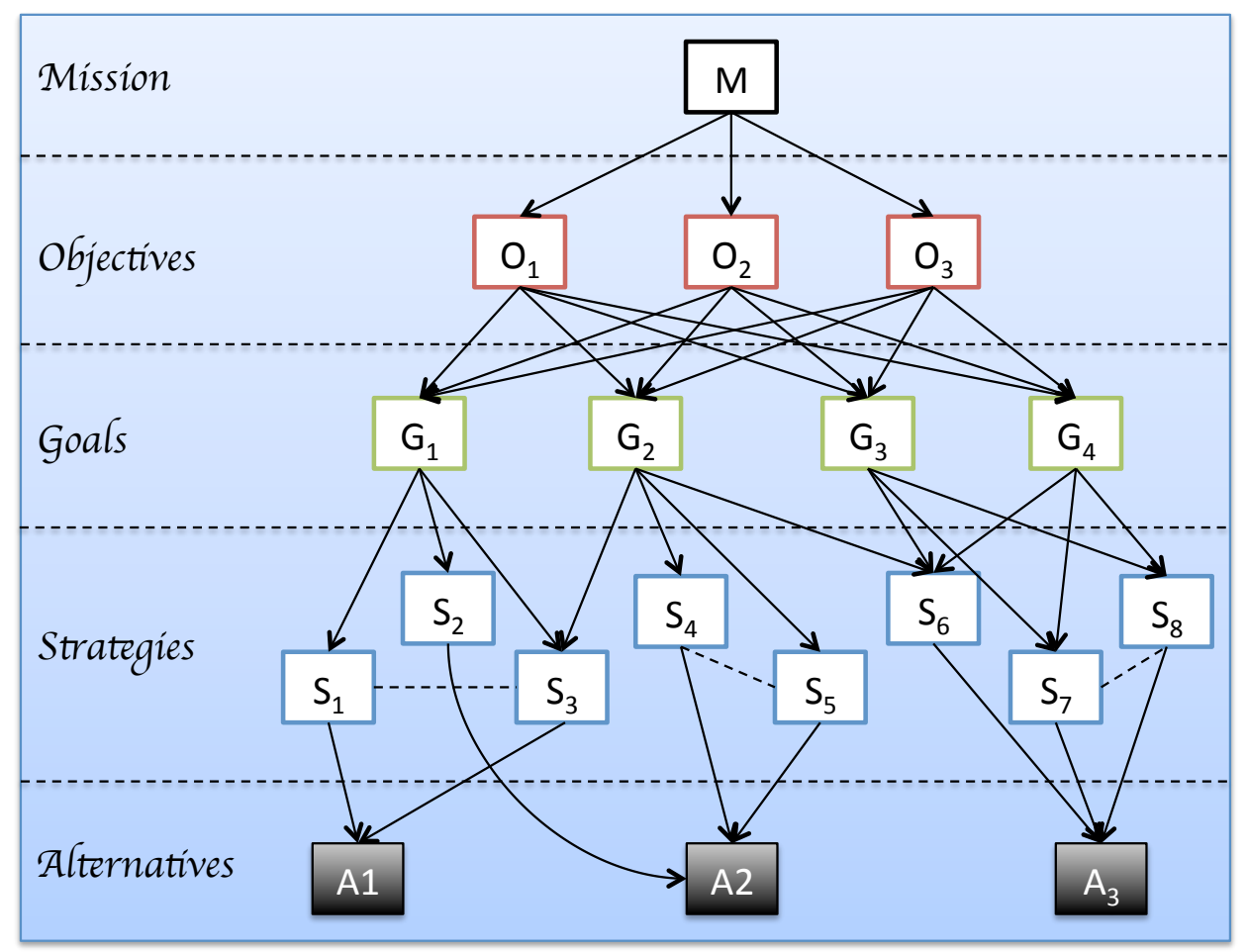

Figure 6 - MOGSA Structure, Adapted from [241]

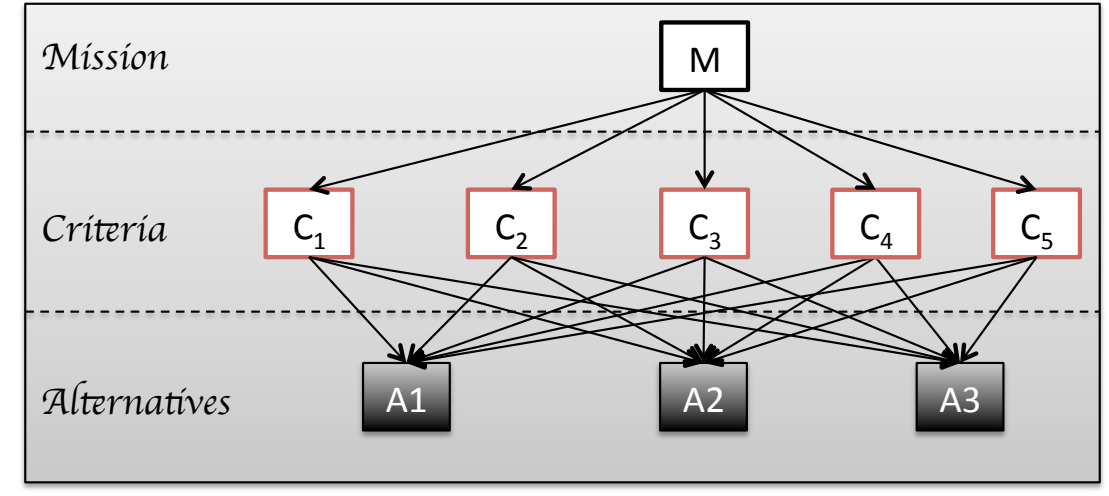

Figure 7 - Three Level HDM

As stated earlier, HDM has been applied in several different settings and fields, proving that it is, indeed, an effective method. The fields and areas that were explored using HDM are, but not limited to, computer selection [237], agriculture [241], 
university housing [236], selection of graduate school [240], transportation options [242], solar photovoltaic technologies [239], health technology assessment [243], semiconductors industry [238], energy [244], and technology transfer [245], among others.

Engineering and research managers are frequently faced with multilevel decisions under conflicting objectives and criteria. They develop technical strategies to fulfill multiple goals, allocate resources to implement multiple strategies, and to evaluate their projects and programs in terms of time, cost, and performance characteristics [235]. As the world has become more complex, decision problems have followed suit and organizations must contend with increasingly complex relationships and interactions among the decision elements. To assist decision makers and analysts, different methods have been developed to breakdown problems into hierarchical levels and to formulate hierarchical decision models (HDM) [246]. As Taha et al. states, the decision process is as important as the decision itself. Thus, choosing the right method to aid in the decision process can be the difference between success and failure [237]. Still according to the same authors, the best decision model to use when subjective judgment is needed to evaluate and select a solution with many criteria is the Hierarchical Decision Model (HDM) [237].

The HDM method is very similar to the Analytic Hierarchy Process (AHP), developed by Saaty [247]. Both methods are significantly similar in their approach and structure, and they produce nearly identical results. In truth, HDM can be regarded as a variant of AHP [248]. There is a difference between the two methods when it comes to the measurement of logical inconsistencies among experts' 
judgments. In HDM, a metric based on variance is used, and in AHP, the consistency ratio is used [249]. Notwithstanding the slightly different approaches, the managerial recommendation was the same in both cases, i.e., to reject any inconsistencies above the $10 \%$ threshold. In 2016, however, Abbas created a novel method for measuring and treating inconsistency, one that uses statistical hypotheses testing, uses the number of alternatives as a function, and uses the root of the sum of variances (RSV) to give different acceptable thresholds for different confidence levels - [248].

The most notable differences between the two methods lie in the way the pairwise comparisons are conducted in terms of the scale used and the mathematical computation used to rank the alternatives. Kocaoglu's HDM utilizes the constant-sum method (dividing a total of 100 points between each variable of the pair) to account for the differences in importance between model components [250], whereas Saaty's AHP utilizes a nine-point scale and the right eigenvector [251]. According to Abbas, both methods are interchangeable, i.e., any decision-making problem tackled by AHP can also be tackled by HDM and vice-versa, and results would be similar, but Kocaoglu's method would yield more accurate, flexible, and fast results due to the scale used [248]. Additionally, the greater granularity of the constant-sum method has been praised for offering better control in the importance estimates [252]. Previous research has criticized Saaty's scale, particularly concerning its discretization and how it could negatively influence results [253], [254].

The concept of desirability functions is used to calculate the technology transfer score. Desirability functions are the assignment of desirability values (or usefulness values) for a range of possible situations (or performance characteristics) 
for each factor. In other words, it is an attempt to embed the utility curve of each factor into the model. For each of the factors in the model, levels (or metrics) are set and experts are prompted to assign a desirability value for each of those levels between 0 and 100 (with 0 representing the least desirable situation and 100 representing the most desirable situation). The desirability values are used to plot the desirability curves. The curves' distribution will change depending on the nature of each factor. For instance, let us imagine two factors in a model: Percentage of PhDs in the R\&D department and number of hours employees work per day in the R\&D department. The levels could be defined as follows:

- $\%$ of PhDs in the R\&D department

○ $\quad 0 \% ; 20 \% ; 40 \% ; 60 \% ; 80 \% ; 100 \%$

- Number of hours employees work per day

○ $2 \mathrm{~h} ; 4 \mathrm{~h} ; 6 \mathrm{~h} ; 8 \mathrm{~h} ; 10 \mathrm{~h} ; 12 \mathrm{~h} ; 14 \mathrm{~h}$

Experts evaluate the levels in light of the factors and assign values from 0 to 100 to each level. Since for an R\&D department the more PhDs the better, experts could assign the following values:

- \% of PhDs in the R\&D department

$$
\begin{array}{ll}
\circ & 0 \%-0 \\
\circ & 20 \%-10 \\
\circ & 40 \%-30 \\
\circ & 60 \%-50 \\
\circ & 80 \%-75
\end{array}
$$


In this case, as shown in Figure 8, the desirability curve for the factor "Percentage of PhDs in the R\&D department" would look like the following:

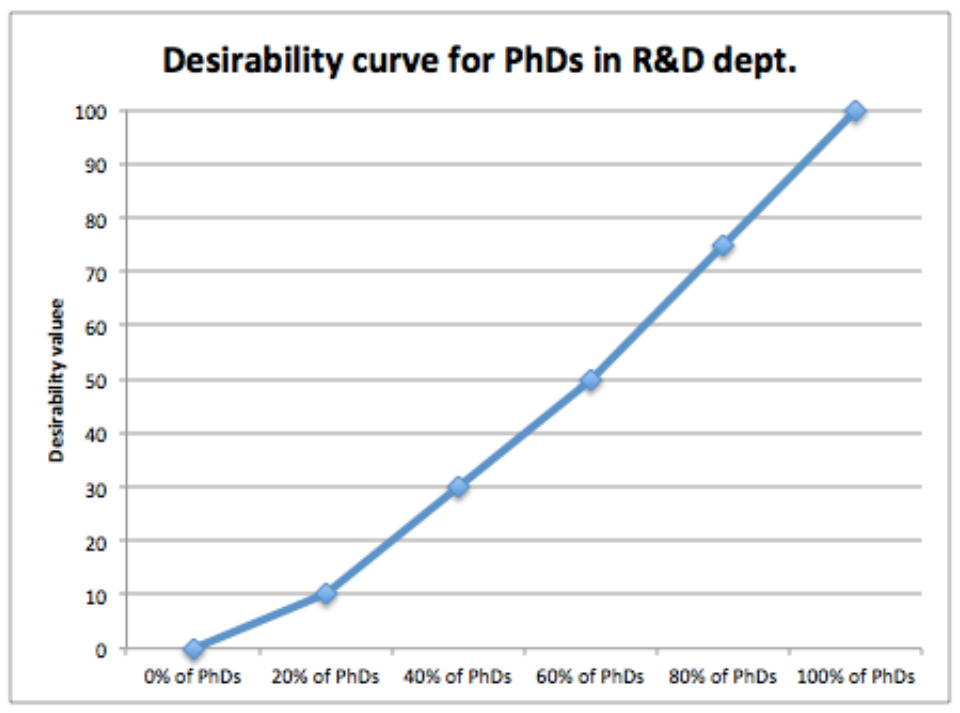

Figure 8 - Desirability Curve - Example 1

For every hour worked, workers become tired, so after a certain point their efficiency and well-being are likely to fall rapidly. With that in mind, experts could assign the following values to the second factor:

- Number of hours employees work per day
$\circ 2 \mathrm{~h}-0$
○ $4 h-25$
○ $6 h-60$
○ $8 h-100$ 


$$
\begin{aligned}
& \text { ○h - } 60 \\
& \circ 12 h-30 \\
& \circ 14 h-0
\end{aligned}
$$

In this case, as shown in Figure 9, the desirability curve for the factor "Number of hours employees work" would look like the following:

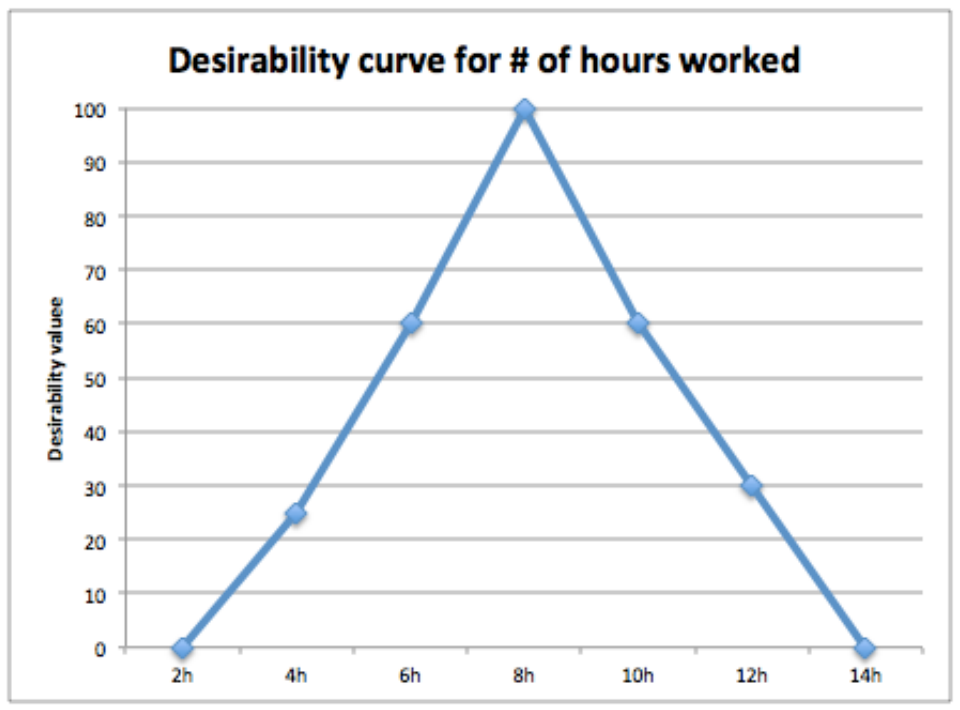

Figure 9 - Desirability Curve - Example 2

The great advantage of using desirability functions is the flexibility they provide to the model. After gaining experts' judgments on each factor through the desirability values, one can replicate the model and apply it again and again using different alternatives, without having to go back and consult with the experts. This is provided, of course, that the weights of perspectives and factors remain unchanged. Conversely, if one does not use desirability functions, one would have to go back to the experts and start the quantification process anew with every change in the alternatives. Moreover, using desirability curves provides the researcher with the 
advantage of more properly capturing the non-linearity nature of some factors, such as in the example given above in Figure 9. Additionally, desirability curves are able to more accurately capture the dynamics or granularity of each factor, i.e. it properly portrays the pattern of variability within each factor in terms of utility, be it linear, bell-shaped, etc.

According to the HDM methodology developed by Kocaoglu [250], pair-wise comparisons are made between each item in every layer of the model. After conducting the pair-wise comparisons, normalized matrices are generated with the expert judgments. The importance of every component of a given layer relative to the layer right above it is extracted by averaging the rows of the normalized matrices. The importance of every model component relative to the first layer (or the global importance) is calculated by multiplying its local importance (relative only to the layer above it) by the importance of its "parents" relative to the first layer. Bringing this rationale of using three layers to this study's model, the calculation of the factors' importance relative to the mission, the Organizational TT score, is given by the following equation:

$$
S_{n, j n}^{T T}=\sum_{n=1}^{N} \sum_{j n=1}^{J n}\left(P_{n}^{T T}\right)\left(F_{n, j n}^{P}\right)
$$

Where:

$S_{n, j n}^{T T}=$ Relative value of the $\mathrm{j}_{\mathrm{n}}{ }^{\text {th }}$ factor under the nth perspective with respect to the TT score. 
$P_{n}^{T T}=$ Relative priority of the $\mathrm{n}^{\text {th }}$ perspective with respect to the TT score, $\mathrm{n}=1,2,3 \ldots \mathrm{N}$. $F_{n, j n}^{P}=$ Relative contribution of the $\mathrm{j}_{\mathrm{n}}{ }^{\text {th }}$ factor under the $\mathrm{n}^{\text {th }}$ perspective, $\mathrm{j}_{\mathrm{n}}=1,2,3 \ldots \mathrm{N}$.

After having the importance of each factor relative to the mission, the determination of the Organizational TT score is given by multiplying the global importance of each factor by its desirability value, and making the total summation, as shown in the following equation:

$$
\text { Org TT Score }=\sum_{n=1}^{N} \sum_{j n=1}^{J n}\left(S_{n, j n}^{T T}\right)\left(D_{n, j n}\right)
$$

Where:

$S_{n, j n}^{T T}=$ Relative value of the $\mathrm{j}_{\mathrm{n}}{ }^{\text {th }}$ factor under the nth perspective with respect to the TT score.

$D_{n, j n}=$ Desirability value of the performance measure corresponding to the $\mathrm{j}_{\mathrm{n}}{ }^{\text {th }}$ factor under the $\mathrm{n}^{\text {th }}$ perspective.

Obviously, every method has its strengths and weaknesses, its advantages and limitations. One of the weaknesses of using HDM is that in order to apply the HDM, it is mandatory to find and have access to experts. If, for one reason or another, it is not possible to count on good experts in the specific research field, the outcome may be seriously compromised. Moreover, because the analyses are based on pair-wise comparisons, as the number of considered alternatives rises, the method 
effectiveness decreases. This is due to the fact that the experts need to concentrate and make time-consuming choices and decisions to get to a final conclusion regarding a comparison, which means that after a certain amount of time they may eventually feel tired and lose concentration. Thus, the more comparisons one expert has to do, the less concentrated he/she will be, and the less accurate his comparisons will be. The following are the most important limitations of the method:

- Subjectivity and biases

○ HDM transforms experts' qualitative judgments into quantitative figures in order to rank alternatives and create a score. Since those qualitative judgments are made subjectively according to the expertise of each subject matter expert, the lack of objectivity can be seen as a challenge. Also, as all of the experts are human, biases are going to be present, and can represent a threat to achieving solid results if not treated properly.

- The most effective way to deal with this challenge is to form solid and trustworthy expert panels, by choosing the right experts and sorting them according to their expertise, as to maximize their contribution to the model. There are guidelines on how to properly choose experts and form the panels, thereby minimizing this challenge.

- Inconsistencies / disagreements

- Closely related to the fact that HDM heavily relies on expert judgments, comes the challenge of inaccuracies, logical inconsistencies, and 
disagreements. Again, as all of the experts are human, one should expect them, to a certain degree, to be inaccurate, logically inconsistent, and for there to be disagreement among them. These challenges are impossible to eliminate. Instead, a good HDM application accurately measures the levels of inconsistency and disagreement, contrasting those against pre-established acceptable levels. In case there is too much disagreement or inconsistency, there are ways to mitigate these problems.

- Changes in weights might change the results

- Another important limitation of HDM is its lack of flexibility and adaptability regarding changes. It is unlikely that the problem the researcher is trying to tackle will be immune to changes, be it changes in the priorities (importance) of the model components, changes in the application area, changes in the model components, changes in the required experts, etc... These changes could happen gradually overtime or even happen abruptly (e.g. senior management radically changing strategy in a company will certainly alter the importance of criteria in a model). Given the mechanism through which HDM works, any of those changes will likely produce alterations in the final results. Ideally, a model would be able to quickly adapt to these changes and provide new results accordingly. Nonetheless, when using HDM, the researcher has to start its model anew to account for the changes. Although there 
are no definitive solutions to this limitation, sensitivity analysis is a good way of being aware of how robust the model is and how well it would respond to these changes thus providing the researcher with enough information to realize when the model should be altered.

As opposed to its weaknesses and limitations, the HDM method has several advantages and strengths. For instance, if the decision maker finds and has access to experts, all the reasoning and analyses will rest upon their opinions, which makes the outcome trustworthy. Furthermore, one of the biggest barriers in the decisionmaking process is to translate qualitative data into quantitative information. HDM is a good method to do this because it has the ability to interpret subjective aspects of a problem in order to analyze it objectively.

\section{Other Approaches and Why Choose HDM}

Although a myriad of methods could possibly be applied to technology transfer issues, it is prudent and reasonable to limit this analysis to those methods and approaches that have been used by researchers in previous studies. Both quantitative and qualitative methods are used to tackle technology transfer issues. Quantitative methods would include tools focusing on mathematical and statistical models, plus Multi-Criteria Decision-Making Models (MCDM), such as Analytical Hierarchical Process (AHP) and Hierarchical Decision Modeling (HDM). According to Khabiri et al., qualitative methods define activities of those who are involved in the process, and elicit factors and issues that may influence the success and effectiveness of a TT 
project [53]. On the other hand, quantitative methods would quantify parameters and analyze them.

Table 25 summarizes some of the sources found in the literature that use and/or mention methods for technology transfer:

Table 25 - Technology Transfer Method Types

\begin{tabular}{|c|c|}
\hline \multicolumn{2}{|c|}{ Technology Transfer Method Types } \\
\hline Type & References \\
\hline Quantitative & {$[6],[25],[36],[51],[59],[61],[87]-[90]$} \\
\hline Qualitative & {$[2]-[4],[7],[22],[23],[34],[35],[38],[41]$,} \\
& {$[54]$, [63] } \\
\hline
\end{tabular}

Table 26 summarizes some of the sources found in the literature that use and/or mention specific methods and tools for technology transfer studies:

Table 26 - Technology Transfer Methods and Tools

\begin{tabular}{|c|c|c|}
\hline \multicolumn{3}{|c|}{ Technology Transfer Methods and Tools } \\
\hline Method & Type & Reference \\
\hline AHP; HDM & Quantitative & [6], [19], [90] [91] \\
\hline Decision-Model & Qualitative & {$[2],[22],[34]$} \\
\hline Fuzzy-Set Theory & Quantitative & {$[90]$} \\
\hline Interviews & Qualitative & {$[63]$} \\
\hline Mathematical Models & Quantitative & {$[87],[89]$} \\
\hline Other Types of MCDM & Qualitative & {$[3],[4],[7],[35],[38],[41]$} \\
\hline Nonlinear Differential Equation & Quantitative & {$[25]$} \\
\hline
\end{tabular}




\begin{tabular}{|c|c|c|}
\hline Scenario Analysis & Qualitative & {$[25]$} \\
\hline Maturity Scale & Qualitative & {$[91]$} \\
\hline Social Network Analysis & Qualitative & {$[92]$} \\
\hline
\end{tabular}

As it can be seen in Table 26, subjective models are, by far, the dominant way of dealing with technology transfer. Within the subjective models, Multi-Criteria Decision-Making (MCDM) methods are frequently used. As previously mentioned, technology transfer is a complex, multi-faceted, and multi-disciplinary effort. As such, it seems that decision models and especially MCDM models are the most appropriate methods, since their strength is on the fact that they can approach the problem from several different perspectives at the same time. Not only are AHP [90], [91] and HDM [6], [19] used, but also decision models based on donor/recipient criteria [2], [22], [34]; manufacturing strategy models [41]; contingent effectiveness models [3], [7]; broadcasting models [38]; multi constituency models [35]; climate-friendly technology transfer models [4].

Should the researcher choose to use more mathematics-leaning methods, such as non-linear differential equation, fuzzy set theory, or any other mathematical models, the most important advantage would be the objectivity of those methods. Mathematical models would provide the researcher with much more consistent and robust results than an expert-based model, as the concerns about biases, logical consistency, and disagreement would not be present.

The strength of working with scenarios is the ability to prepare for different contingencies and circumstances. Regarding technology transfer, this ability would 
be especially interesting when planning for the deployment of different transfer mechanisms, depending on the scenario that becomes reality.

Interviews are an interesting data collection approach that can be used on virtually any issue involving human subjects. It is powerful enough to gather high fidelity information about how important stakeholders feel about something or the way they regard a certain issue, and that information can be used to explain or describe issues or even to formulate hypotheses to be later tested through data analysis methods. In turn, maturity scales are very useful and have their biggest strength in creating an ideal situation (the most mature level of the scale) towards which practitioners can aim their actions.

Lastly, social network analysis has been successfully used in a wide variety of applications. Its main strength, for technology transfer purposes, is being able to easily and quickly identify and measure interactions and interdependencies between different technologies and players, in a way that no other method can. It is used to identify possible stakeholders to collaborate with and potential donors and/or recipients. Also, the method can be used to trace interactions and interconnections with other fields and past technologies, aiming to choose the best transfer mechanism for the technology being developed.

Taking into consideration all of the advantages of the methods listed above, it is also important to highlight their weaknesses and limitations that would negatively impact a technology transfer study.

Mathematical models, as much as they are objective and precise in their results, cannot absorb and translate the human complexities involved in technology 
transfer issues, and their results may not be easily interpretable and meaningful to practitioners.

Interviews, as already discussed, are a good data collection method, but they require another method to analyze the gathered data, if one wishes to go beyond merely describing the situation/problem. Therefore interviews, on their own, are not enough to tackle technology transfer issues. A similar limitation applies to maturity scales. These, applied as a stand-alone item, give important information to managers, but not quite enough to create the awareness and knowledge necessary to improve a technology transfer process. They would provide practitioners with standardized levels of excellence in a certain area, but without the aid of another method they would leave the practitioner lost on how to contrast the organization's practices against the pre-established levels, not to mention the lack of guidelines on how to go about moving up the maturity ladder.

Scenarios, although useful for preparation purposes, do not have a strong enough analysis component to evaluate an organization's practices, and thus are insufficient to tackle the intended technology transfer situation. Similarly, social network analysis is not a good choice to analyze an organization's processes either. Additionally, SNA studies usually give information on a narrow scope, such as. collaborative R\&D on a specific topic through co-authorship or join patents networks, but it fails to consider multiple instances and perspectives, and fails to provide a holistic view of the problem.

Some of the decision models used in previous research do consider multiple perspectives that would affect technology transfer. But, generally those models stop 
at gathering qualitative data from literature or from experts, They would bring a detailed portrait of the problem, with all its complexity, but fail to go beyond the description and actually analyze the problem, and fail to make suggestions and recommendations.

Having gone through the main strengths and weaknesses of the methods that could be used to tackle a technology transfer issue, one can confidently state that an HDM model is the most indicated method to be used. The following are some points that summarize this reasoning:

- Action research as an aid

- The model is strengthened by the aid of action research, making it an even more appropriate method for the problem to be tackled.

- TT is a complex and multidisciplinary issue.

- Multiple perspectives are required to comprehensively tackle the issue, and HDM is ideal to merge multiple perspectives into a single framework / model.

- HDM transforms qualitative judgments into quantitative measures

- Qualitative approaches may serve in helping practitioners, but they do not help them in a way that measures the effectiveness of their processes, nor as a guide on how to improve these processes. The methodological characteristics required to properly tackle a technology transfer issue are seemingly paradoxical, and are difficult to find in a method. TT is a complex issue, and needs an approach that 
captures experts' judgments (qualitative data) and also needs a quantitative processing for those judgments in order to provide clear and meaningful results to practitioners. HDM does just that.

- $\quad$ HDM is easily applied

- Notwithstanding its quantitative features, and the laborious and meticulous process through which the model is composed, validated, and quantified, the concept behind HDM is very easily grasped and the model's results are also easily interpreted. This is especially true when compared to other mathematically-based methods, and thus it is seen with more welcoming eyes from experts and practitioners.

- HDM is a data analysis method

○ Other approaches involving only data gathering might be enough to describe a certain situation, but not to analyze it. HDM goes beyond the mere description of a problem (the model itself and its components), and provides an analysis based on the problem's description and the researcher's intended objective (the outcome of the model).

\section{Research Framework}

Figure 10 is a chart illustrating the approach used. 


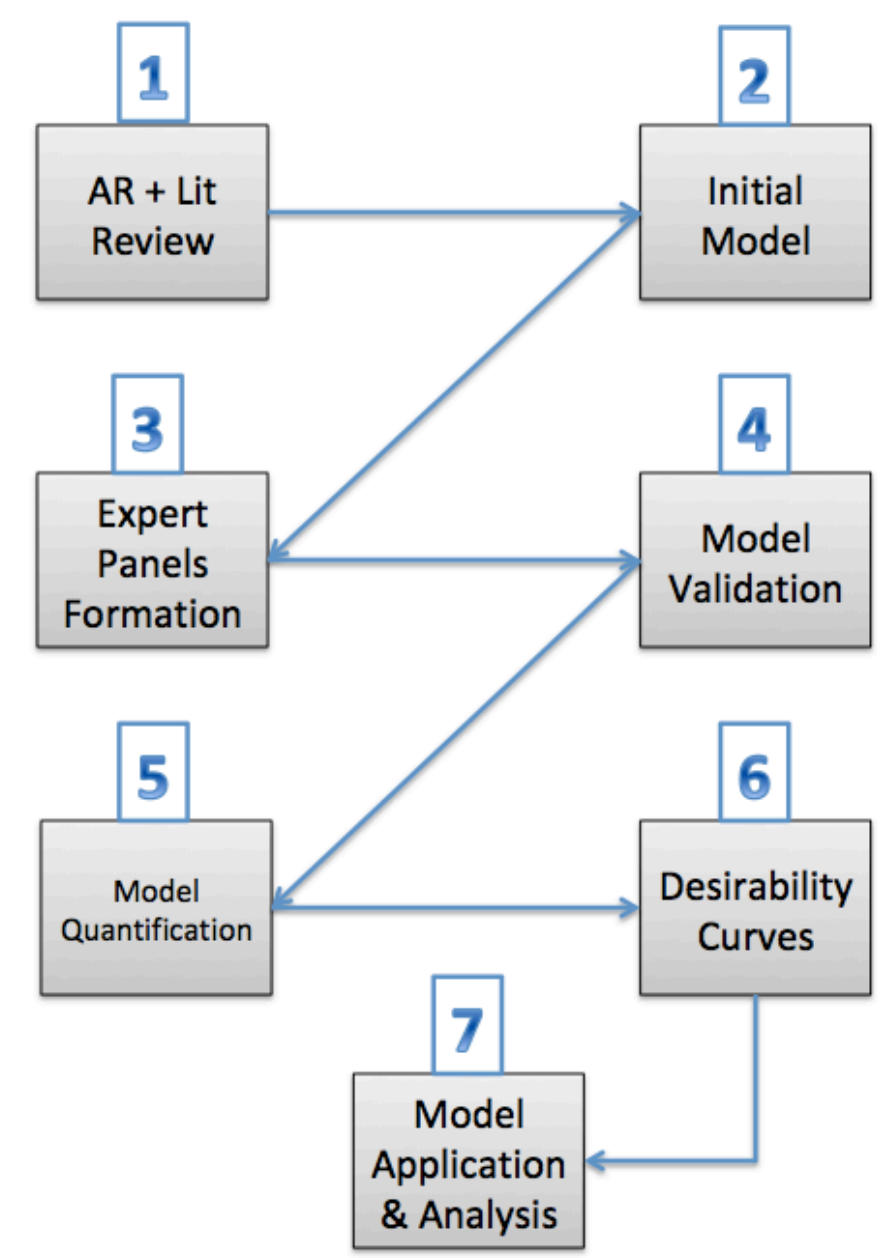

Figure 10 - Research Approach

\section{$1^{\text {st }}$ Step - AR and Literature Review}

As previously explained, an action research project was conducted, and its results were used to aid in the initial model development. Simultaneously, a literature review was made regarding important criteria for the model, and criteria derived from the action research were contrasted against the literature. 
$2^{\text {nd }}$ Step - Initial Model

An HDM model containing input from both the literature and the action research was built.

\section{$3^{\text {rd }}$ Step - Experts Panel Formation}

Experts were identified to provide input to the model, by validating the initial model and then quantifying it.

According to the Cambridge dictionary, an expert is "a person having a high level of knowledge or skill in a particular subject" [255]. Expert panels are a group of experts who are summoned to gather and discuss a subject or provide a service, for example feedback or recommendations [256]. In 2005, Dreyfus and Dreyfus published a study describing a five-stage model that explains the process of expertise acquisition, i.e. the process a person goes through in order to become an expert in a subject [257]. The five stages are briefly explained below:

- $\quad$ Stage 1 - Novice: The person, having no previous experience or knowledge in the subject whatsoever, has contact with the basic rules, conditions, and environment surrounding the studied subject.

- Stage 2 - Advanced beginner: The novice, having now a little experience, starts to develop a wider understanding about the subject and to notice different features and aspects of the studied subject.

- Stage 3 - Competence: The beginner understands how complex the subject is, and can be overwhelmed with the amount of information, complexity, and 
skills involved in mastering the subject. He/she breaks the subject down in smaller parts and tackles them separately, gradually evolving and developing skills in each of the parts at a time.

- Stage 4-Proficiency: Competence reaches a level at which responses to some situations become automatic and intuitive rather than reasoned. Actions and decisions are taken faster and without a long and structured line of thought or considerations.

- Stage 5 - Expertise: The expert has a higher level of refinement as compared to the proficient. He/she can react faster, and has immediate intuitive response for complex situations.

The use of expert panels in some fields is widespread e.g., health sciences, and it can be a valuable resource for researchers, when compared with doing only a literature review on a subject [258]. According to Holman et al., expert panels are valuable for bringing different perspectives and points of view to the discussion, but potential biases would be a problem of the approach [259]. According to Knol et al, expert panels can be used to assess poorly known parameters and also to discuss and develop qualitative issues, including the creation of conceptual models [260]. The authors also highlight the value of expert panels in enhancing the research, bringing betterments to the assessment being conducted [260]. Reaffirming this point, Morgan states that the experts' knowledge bring more confidence and reliability to the judgments needed for the research [261]. 
The most important challenges in working with expert opinions are the potential biases and their overconfidence in judging subjects and situations they know well [262]. In the words of Morgan, "because experts are human, there is simply no way to eliminate cognitive bias and overconfidence" [261, p. 7183]. Identifying and recruiting the best experts to the situation, and at the same time making sure the results of the panel are reliable is also a significant challenge [263], [264]. Balancing the experts panel is also a concern, and to make sure each panel represents a robust and significant sample of the existent knowledge on the field [263].

The size of the panels is a major concern as well [263]. Commenting on the Delphi method, Phan states that the most recommended size of an expert panel would be from 10 to 15 experts [265]. Nonetheless, successful studies have been conducted utilizing sub-groups of experts as small as five members [266] or three members [264]. Since dealing with a large number of experts augments the process complexity exponentially, it has been argued that the maximum amount of experts per panel should be 12 [260]. Leveraging the work done in past dissertations [245], [263], [264], [266]-[268], it is safe to say that expert panels composed of six to twelve experts each are reliable and at the same time manageable.

Experts should be selected taking into account several aspects, such as how much of an expert the person is, i.e. what were the contributions and the significance of their contributions to the field of study; minimizing the bias as much as possible, i.e., checking if the selected experts have any special reason or personal interest that would enhance the bias potential; how available or willing are the experts, i.e., not 
only the person should be an expert, but also he/she should be willing to fully participate in the study, as to spend enough time and attention taking care of their tasks as an expert [263], [266], [267].

The process of selecting the expert is not trivial, due to the challenges aforementioned. The most proper methods to choose experts, according to Tran, are the use of personal connections-if the researcher has easy access to knowledgeable people in the field; snowball sampling - the researcher starts with a small group of experts, which in turn recommend more expert and so on; social network analysis the researcher draws a network based on collaborations, co-authorship or citations in order to discover the most relevant and influential actors in the field of study [264].

\section{$4^{\text {th }}$ Step - Model Validation}

The model validation step is illustrated by Figure 11.

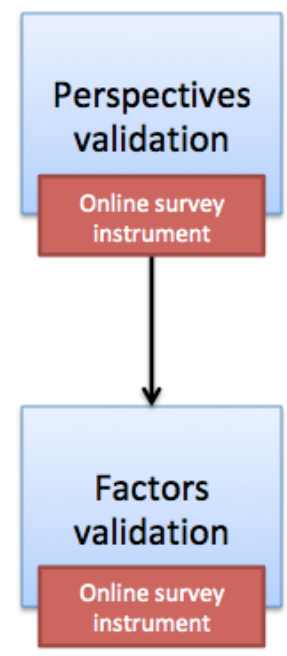

Figure 11 - Model Validation Framework 
Two survey instruments were created and sent to the experts by email, along with attachments explaining the objectives of the research, and explaining the model. Firstly, a survey explaining the model perspectives was sent and experts validated them. Next, a survey explaining the model factors was sent and experts validated them.

\section{$5^{\text {th }}$ Step - Model Quantification}

The model quantification step is illustrated by Figure 12.

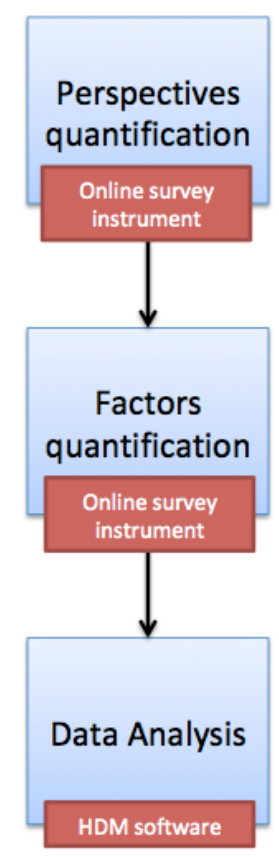

Figure 12 - Model Quantification Framework

Two instruments were created and sent to the experts by email, along with attachments explaining the objectives of the research and explaining the model. Firstly, an online survey instrument was sent, and experts quantified the model's 
perspectives. Next, another online survey instrument was sent, and experts quantified the model's factors. After gathering the data, the HDM ${ }^{\circledR}$ software was used to compile and analyze the experts' judgments, arriving to the final model weights.

\section{$6^{\text {th }}$ Step - Desirability Curves}

The desirability curves were created with the aid of a senior management in a large power utility in the Pacific Northwest, which has solid experience technology transfer both in academia and in the industry. The executive was presented with the desirability levels, and after in-depth discussions, the levels were validated and quantified, generating the curves that will be presented later in this dissertation.

\section{$7^{\text {th }}$ step - Model Application and Analysis}

As the research approach last step, the model was applied using Bonneville Power Administration's current TT process as a case. The identification of BPA's process situation within each factor-leading to the determination of the desirability values - was done based on access to BPA's internal data, and the author's knowledge about the process, coupled with consultations with BPA managers. After the model was applied, sensitivity analysis was performed in order to check and understand the impact on the total TT score due to changes in the priorities of model perspectives. The following is an explanation of possible inconsistency, disagreement, and sensitivity analyses. 


\section{Inconsistency Analysis}

The inconsistency analysis is one of the key data analysis items in applying the HDM methodology [264]. According to Estep, "generally, inconsistency can be defined as disagreement within an individual's evaluation" $[245, \mathrm{p} .75]$. In the words of Abotah, "inconsistency is a measure that explains how reliable and homogeneous in his or her answers each expert was through the whole questionnaire" [263, p. 65]. In other words, the inconsistency of an expert can be thought of as the logical incoherence of his/her judgments. For instance, given three factors A, B and C, if A is better than $B$ and $B$ is better than $C, A$ must be better than $C$ if one is to be logically consistent (ordinal consistency). Moreover, if A is two times better than B, and B is three times better than $C$, then A must be six times better than $C$, if one is to be logically consistent (cardinal consistency). Chan argues that inconsistencies in experts' judgments are common in AHP-based studies [266]. Following the same reasoning, Gibson states that one should expect inconsistency to occur when experts face multiple decisions and have to judge items [268].

In more technical terms, I quote the words of Phan from his PhD dissertation in 2013:

"For $\mathrm{n}$ elements, the constant sum calculation results in a vector of relative values $r 1, r 2, \ldots, r n$ for each of the $n$ ! orientations of the elements. For example, if three elements are evaluated, $n$ is 3 , and $n$ ! is 6. The 6 orientations would be $\mathrm{ABC}, \mathrm{ACB}, \mathrm{BAC}, \mathrm{BCA}, \mathrm{CAB}$, and CBA. If an expert is consistent in providing pairwise comparisons, the relative values are consistent for each orientation. However, if an expert is inconsistent in providing pairwise comparisons, the relative values are inconsistent for each orientation. The inconsistency in this methodology is measured by the variance among the relative values of the elements calculated in the n! orientations." [265, p. 45]. 
The formulas to calculate the inconsistency level are the following, adapted from [250], [263], [265]:

Let:

$r_{i j}=$ relative value of the ith element in the $\mathrm{j}^{\text {th }}$ orientation for an expert.

$\bar{r}_{i}=$ mean relative value of the $\mathrm{i}^{\text {th }}$ element for that expert.

$$
\frac{1}{n !} \sum_{j=1}^{n !} r_{i j}
$$

Inconsistency in the relative value of the $\mathrm{i}^{\text {th }}$ element is:

$$
\begin{gathered}
\sqrt{\frac{1}{n !} \sum_{j=1}^{n !}\left(\bar{r}_{i}-r_{i j}\right)^{2}} \\
\text { for I }=1,2,3 \ldots \mathrm{n}
\end{gathered}
$$

Variance of the expert in providing relative values for the n elements is

$$
\text { Inconsistency }=\frac{1}{n} \sum_{i=1}^{n} \sqrt{\frac{1}{n !} \sum_{j=1}^{n !}\left(\bar{r}_{i}-r_{i j}\right)^{2}}
$$

As noted by Kocaoglu [250], and as per precedent established by other studies [245], [263], [264], [266], [268], the inconsistency level should not be higher than $10 \%$, in order to be taken as acceptable. Should the inconsistency level exceed the 10\% mark, a more careful consideration should be taken, e.g., the most inconsistent experts should be asked to repeat the judgments, and in extreme cases the most 
inconsistent judgments could be deleted from the analysis [268]. Additionally, in case of large inconsistencies, another method of calculating the inconsistency could be used to further analyze the matter, such as the root-sum of variances created by Abbas [248]. His method utilizes the root-sum of the variances (RSV), and it takes into account the number of pair-wise comparisons experts are making. The formulas below depict the calculations used that were adapted from [248].

$$
R S V=\sqrt{\sum_{i=1}^{n} \sigma_{i}^{2}}
$$

Where:

HDM inconsistency $=$ Root of the Sum of Variances (RSV)

$\sigma_{i}{ }^{2}=$ variance of the mean of the $i^{\text {th }}$ decision element

$$
\sigma_{i}=\sqrt{\frac{1}{n !} \sum_{j=1}^{n !}\left(x_{i j}-\bar{x}_{i j}\right)^{2}}
$$

Where:

$x_{i j}=$ normalized relative value of the variable $\mathrm{i}$ for the $\mathrm{j}^{\text {th }}$ orientation in $\mathrm{n}$ factorial orientations

$\bar{x}_{i j}=$ mean of the normalized relative value of the variable $i$ for the $j^{\text {th }}$ orientation

$$
\bar{x}_{i j}=\frac{1}{n !} \sum_{j=1}^{n !} x_{i j}
$$

Where:

$\bar{x}_{i j}=$ mean of the normalized relative value of the variable i for the $j^{\text {th }}$ orientation 
$x_{i j}=$ normalized relative value of the variable $\mathrm{i}$ for the $\mathrm{j}^{\text {th }}$ orientation in $\mathrm{n}$ factorial orientations

\section{Disagreement Analysis}

The disagreement analysis is also noted as one of the key data analysis items in applying the HDM methodology [264]. In the words of Tran, "the agreement among the experts' judgment is represented by a disagreement value of the expert group in a pairwise comparison procedure" [264, p. 66]. Quoting from Abotah's dissertation, "the disagreement of experts can be understood as the deviation of their judgments from each other" [263, p. 60]. To measure and treat the disagreement levels would be especially important in order to guarantee the significance of the results of experts judgments [264]. It could be problematic if researchers did not check the agreement level between raters before making any data analysis [269].

Although disagreement would be something natural among experts, it should be treated. In case the disagreement level is greater than what is acceptable, another round of judgments could be conducted with the aim of reaching a consensus or quasi-consensus situation (following the Delphi methodology). However, in cases where the vast majority of experts agree but there is one or a few outliers bringing the disagreement level up, a follow-up with the outliers should be conducted in order to check if they have correctly interpreted the components and concepts involved in the study [245]. The removal of those outliers from the pool of experts could also be contemplated as a viable option in extreme cases. 
A common method of measuring the disagreement between experts is to use the PCM group disagreement index, according to the formula below, which is adapted from [263], [265], [267].

$$
d=\sqrt{\frac{1}{m} \sum_{j=1}^{m} \frac{1}{n} \sum_{i=1}^{n}\left(R_{i}-r_{i j}\right)^{2}}
$$

Where:

$R_{i}=$ Group relative value of the $\mathrm{i}^{\text {th }}$ element

$\mathrm{m}=$ number of experts

$\mathrm{n}=$ number of decision variables

$r_{i j}=$ mean relative value of the $\mathrm{i}^{\text {th }}$ element for the $\mathrm{j}^{\text {th }}$ expert

In order to use this method, precedent has it that an acceptable disagreement level would be $10 \%$ or less [245], [263], [267], [268]. Hierarchical agglomerative clustering (HAC) has also been used in previous dissertations to complement the disagreement measurement and interpretation [267], [268]. This technique iteratively groups experts with similar opinions in clusters (or sub-groups) until each cluster's disagreement levels are within acceptable limits, utilizing dendrograms to visually demonstrate the clusters within each expert group.

Two other common methods of measuring the disagreement among experts are the Intraclass Correlation Coefficient (ICC) and the F-test. Several authors have used ICC and F-test as a measurement of disagreement among experts [264]-[266]. 
The ICC would be at the same time a measure of intra-rater reliability and inter-rater reliability, and it is extensively used across different disciplines [269], [270]. A complete agreement between experts would result in the ICC yielding the value 1.00 , while a complete disagreement between experts would result in the ICC yielding the value 0 . It has been argued that an ICC of 0.7 or higher would indicate an acceptable level of agreement [271]. However, there are authors reasoning that the minimum acceptable ICC would vary on a case-by-case basis, heavily depending on the research questions, objectives, and data used [269]. There are several different ways of applying ICC. There are three different models with different options of forms and types, and the researcher should be aware of his/her needs in order to choose the most proper ICC model and its features [269]. Considering the needs of a researcher using HDM and also considering what has been done in previous dissertations [265], the most proper choice of applying ICC would be to use the following model, form, and type:

- Model - Two-way random effects ANOVA

- This choice is made because the same cases will be assessed by all raters, and it is assumed that cases and raters were randomly selected, and raters are representative of other raters. In the words of LeBreton and Senter, "If the researcher were interested in generalizing to other judges, then judges would be treated as a random effects variable, and he or she would calculate the ICC using the two-way random effects 
ANOVA (where both the target and judge effects are random effects)" [270].

- Form - Single measures

- This choice is made because each rater only rates the cases once. The average measures would be selected if each rater were to make multiple measurements per case and the average was being taken, e.g., when nurses take multiple measurements of blood pressure from each patient, and the average is considered for the purpose of the ICC calculation.

- Type - Absolute value

- This choice is made in order to check not only the consistency of the measurements, but also the absolute agreement. In this sense, consistency happens if measurements from different raters vary consistently. Even if the measurements are patently different, absolute agreement happens only if measurements are exactly the same. In other words, the consistency type measures correlation, while the absolute value type measures absolute agreement. For example, if two experts measured desirability values as $10 ; 20 ; 30$ and $50 ; 60 ; 70$ respectively, the ICC using consistency type would be a perfect 1.00 , because both sets of values vary consistently (a 10-point increase between each value). However, the ICC using the absolute agreement type would be less than 1.00 , because the values are not identical. 
According to Trevethan, in order to avoid inflated coefficients the absolute type adds an extra protection layer when measuring disagreement levels. [269].

The ICC is estimated according to the following formula, which is adapted from [270]:

$$
I C C=\frac{M S_{R}-M S_{E}}{M S_{R}+(K-1) M S_{E}+\frac{K}{N}\left(M S_{C}-M S_{E}\right)}
$$

Where:

$M S_{R}=$ mean square for rows (i.e., targets)

$M S_{C}=$ mean square for columns (i.e., judges)

$M S_{E}=$ mean square error all obtained from a two-way ANOVA

$\mathrm{K}=$ number of observations (e.g., ratings or judges) for each of the $\mathrm{N}$ targets

$\mathrm{N}=$ number of targets

The F-test is a statistical test used to compare the ratio of two variances. Some of the assumptions of the test are that the population variances are equal (therefore the null-hypothesis will be that the variances are equal), the population has approximately a normal distribution, and the samples must be independent events [272]. The work done by Shrout and Fleiss in 1979 used ICC as a basis and F-test to check the disagreement levels between raters [273]. It tests a null hypothesis $H_{0}: I C C=0$, meaning that there is no correlation between the values and thus there is an absolute disagreement between the experts. If the null hypothesis is rejected, 
the $H_{1}$ : not $H_{0}$ is confirmed, meaning there is not a statistically significant disagreement between experts. The $\mathrm{F}$ ratio is calculated by the following formula:

$$
F=\frac{M S_{R}}{M S_{E}}
$$

The resulting ratio is then compared with the F-critical value with degrees of freedom $d f_{1}=d f_{R}$ and $d f_{2}=d f_{E}$ at a specific level of confidence (usually $95 \%$ and above). If the calculated ratio is greater that the F-critical value, the null hypothesis can be rejected (at that specific level of confidence), and no significant disagreement between experts would be present.

In this research, the PCM group disagreement index will be used with a maximum allowed level of $10 \%$. For those expert panels where the disagreement level exceeds the maximum allowed value (if any), Hierarchical Agglomerative Clustering (HAC) analysis will be used to identify and understand sub-groups within the panels., It will then analyze the disagreement levels for those sub-groups, and ultimately project how much impact on the final results (TT score) the different judgments of these sub-groups would have.

\section{Sensitivity Analysis}

The impacts of potential changes in the values on the different levels of a model is done by conducting a sensitivity analysis. This test is important because the preset priorities (or weights) of a model's components might change overtime [267], [268] This is especially true in the realm of technology, where changes occur 
extremely rapidly and constantly. Also, changes in the expert panels might bring new priorities (weights), and given the fact that these changes might occur, a sensitivity analysis would be appropriate [245]. The sensitivity analysis shows how strong the decisions and conclusions coming from the model are [263]. It is performed to test and assure the robustness of both the model and the results [267]. The test would also be helpful in understanding how each level of the model and its components relate to each other [266].

In cases where the model's output is the ranking of different alternatives, the sensitivity analysis is especially useful to tell if and how much that original ranking would change due to changes in the priorities of model's components [265], [267]. For instance, the final ranking of the alternatives might be altered if the criteria relevance is altered, and the sensitivity analysis would measure how strong or disruptive these changes would be. Bringing the same reasoning to this study's model, the sensitivity analysis will indicate the changes in the final organizational TT score caused by alterations in the perspective's relevance. This could be particularly interesting if one is to use the model to compare different departments within an organization or to compare different organizations. Moreover, when applying the model to only one case, changing the weights of the perspectives could prompt the technology manager to change his/her reasoning when prioritizing factors to be tackled. For example, if the technical perspective is, by far, the most relevant, weak factors under the technical perspective should be prioritized, even if the organization seems to be performing better in those factors relative to factors under other perspectives. 
Scenarios can be used to test how much the ranking would be altered in a particular setting (for example, if one of the top-level priorities is overwhelmingly more important than the rest), as it was done in previous studies [245], [263]. Notwithstanding the usefulness of testing the sensitivity of a model through different scenarios, in order to calculate how much perturbation in its priorities a model would endure before yielding different results, a more complex method has to be applied. Such a method was created by Chen and Kocaoglu, and it calculates the tolerance of a model to changes, i.e., the allowed range of values within which a contribution can change without altering the final ranking produced by the model [274]. The method was detailed in Chen's dissertation [275], and has been extensively used since then [245], [264]-[267].

The method states that the original ranking of the model will not be changed if:

$$
\lambda \geq P_{i}^{C} \cdot \lambda^{C}
$$

for the perturbation $P_{l *}^{C}$ where

$$
-C_{l *}^{C} \leq P_{l *}^{C} \leq 1-C_{l *}^{C}
$$

and

$$
\lambda=C_{r}^{A}-C_{r+n}^{A}
$$

and

$$
\lambda^{C}=C_{r+n, l *}^{A-C}-C_{r l *}^{A}-\sum_{l=1, l \neq l *}^{L} C_{r+n, l *}^{A-C} \cdot \frac{C_{l}^{C}}{\sum_{l=1, l \neq l *}^{L} C_{l}^{O}}+\sum_{l=1, l \neq l *}^{L} \frac{C_{r l}^{A-O}}{\sum_{l=1, l \neq l *}^{L} C_{l}^{O}}
$$


The allowance range of perturbations $C_{i}^{C}$ to maintain the original ranking is given by:

$$
\left[\delta_{i-}^{C}, \delta C_{i+}^{O}\right]
$$

and the sensitivity coefficient is given by:

$$
1 /\left|\delta_{i+}^{C}, \delta_{i-}^{C}\right|
$$

In this research, the scenario method is used to assess the robustness of the model and its behavior under different and extreme cases.

\section{Research Validity}

Following previous PhD dissertations [245], [263], [266]-[268], the research validity will be guaranteed using three validity measures deployed in distinct points in time during the research, namely content validity, construct validity, and criterionrelated validity.

Content validity is the first measure, and it is used throughout the development of the research model. It refers to the ability of the model contents to properly represent all relevant aspects and elements pertaining to the research topic. In this case, subject matter experts were identified and contacted to validate each element of the model, having the freedom to suggest edits to it, be it to remove items, add new items, or sort and organize items within the model in a different fashion.

Construct validity is the second measure, and it is used after the model is developed. It refers to the fitness of the research approach to past and underlying theories, and also refers to the ability of the model's structure to deal with the 
problem at hand. In this case, the construct validity is tested by ways of consulting with faculty members and doctoral students in the ETM department. Those have accumulated experience in research in the energy realm, and who are also subject matter experts concerning hierarchical decision models.

Criterion-related validity is the final measure, and it takes place after the model is applied and during the results analysis. It refers to the validity of the research outcomes, and the model's ability to accurately portray the situation being studied. In this case, the results were shown to subject matter experts, who had the chance to weigh in and give feedback on the accuracy of the outcomes and the validity of the conclusions and recommendations. 


\section{CHAPTER 4 - RESEARCH MODEL DEVELOPMENT}

\section{Initial Model}

Before presenting the model, its components are listed by source. Table 27 brings the factors coming from the literature review, and Table 28 brings factors coming from the action research.

Table 27 - HDM Factors from Literature

\begin{tabular}{|c|}
\hline Factors in the Model Coming from the Literature \\
\hline TT Team and Training \\
\hline TT Ecosystem Management \\
\hline Conior Management Involvement \\
\hline Absorptive Capacity \\
\hline Long-Range Planning \\
\hline Technology Valuation \\
\hline Proposal Assessment \\
\hline Benefit Management \\
\hline TT Mechanisms Management \\
\hline Business Plan and Use Case \\
\hline Continuity of TT Process \\
\hline
\end{tabular}


Table 28 - HDM factors from Action Research

\begin{tabular}{|c|}
\hline Factors in the Model Coming from the Action Research \\
\hline Stakeholders Management \\
\hline TRL Assessment \\
\hline Data Management \\
\hline Risk Management \\
\hline Outcomes and Decisions \\
\hline Value, Impact and Applicability Management \\
\hline TT Planning, Control and Flexibility \\
\hline Parallel Processes Integration
\end{tabular}

The above listed factors were organized into five perspectives: Human Resources and Stakeholders, Organizational Culture, Technical, Process; and Strategic Alignment. The initial research model is depicted below in Figure 13. 


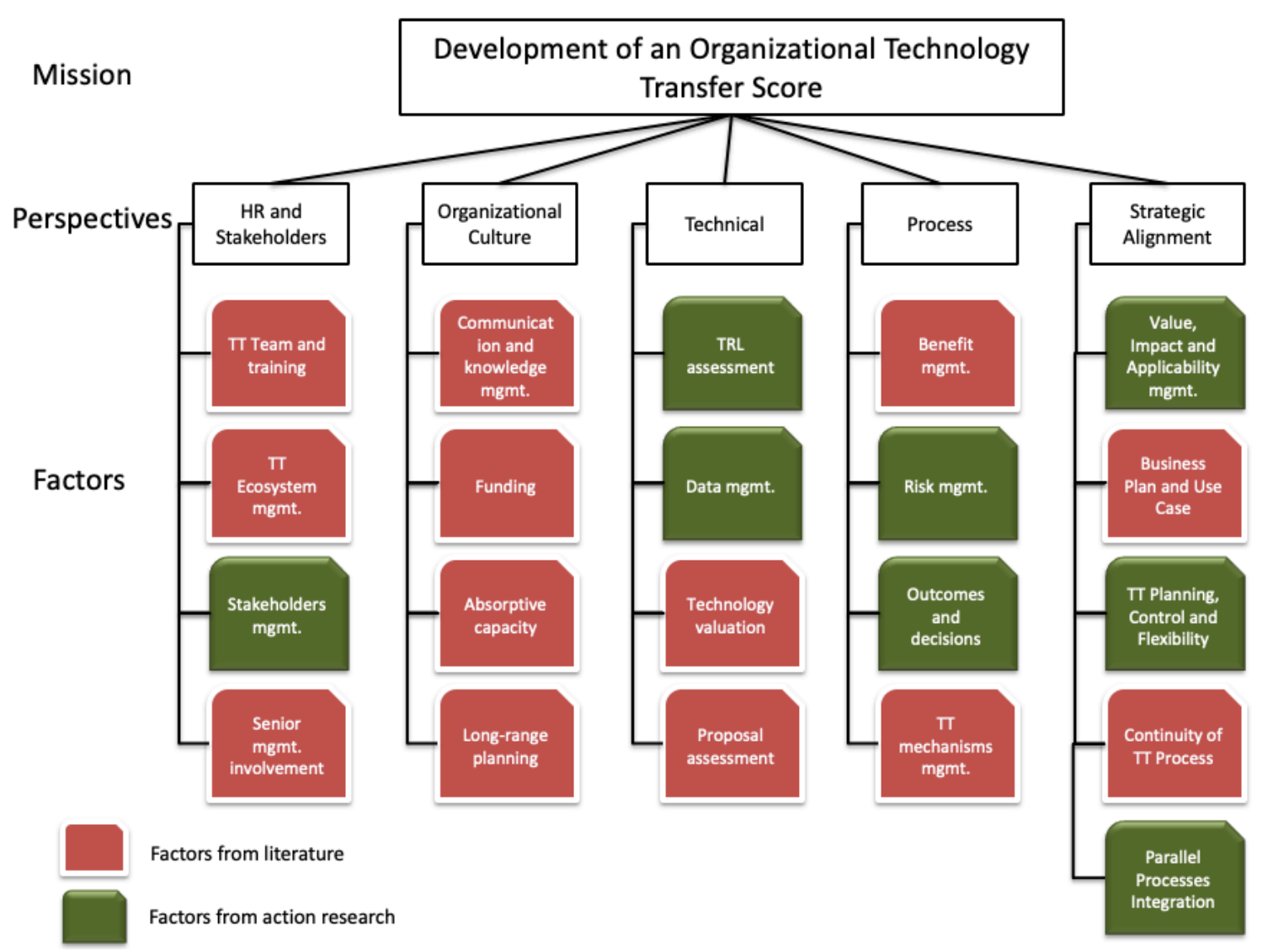

Figure 13 - Initial Model

A brief description of the model components is provided in Table 29 and Table

30.

Table 29 - Description of Model Perspectives

\begin{tabular}{|c|c|}
\hline Perspective & Description \\
\hline Human Resources and Stakeholders & $\begin{array}{l}\text { Factors related to the team, training, ecosystem, } \\
\text { senior management and other players involved. }\end{array}$ \\
\hline Organizational Culture & $\begin{array}{l}\text { Factors related to organizational characteristics } \\
\text { that should be in place for a better TT process. }\end{array}$ \\
\hline Technical & $\begin{array}{l}\text { Factors related to technical assessments and } \\
\text { technology quantifications. }\end{array}$ \\
\hline
\end{tabular}




\begin{tabular}{|l|r|}
\hline Process & $\begin{array}{r}\text { Factors related to the TT process features and } \\
\text { characteristics. }\end{array}$ \\
\hline Strategic Alignment & $\begin{array}{r}\text { Factors related to the alignment between R\&D } \\
\text { and business strategy, and the integration of TT } \\
\text { with other managerial processes. }\end{array}$ \\
\hline
\end{tabular}

Table 30 - Description of Model Factors

\begin{tabular}{|c|c|c|c|}
\hline Perspective & Factor & Description & Source \\
\hline \multirow{3}{*}{$\begin{array}{c}\text { Human Resources and } \\
\text { Stakeholders }\end{array}$} & TT team and training & $\begin{array}{l}\text { Is there a dedicated TT } \\
\text { team? Background of } \\
\text { people in the team; is } \\
\text { the team } \\
\text { multidisciplinary? Type } \\
\text { and frequency of } \\
\text { training. }\end{array}$ & $\begin{array}{c}{[19],[34],[59],} \\
{[91]}\end{array}$ \\
\hline & TT ecosystem mgmt. & $\begin{array}{c}\text { Relationship, } \\
\text { cooperation and } \\
\text { partnership with TT } \\
\text { ecosystem members, } \\
\text { e.g., TTO's, science } \\
\text { parks, research centers, } \\
\text { incubators, POC centers, } \\
\text { etc. }\end{array}$ & $\begin{array}{c}{[19],[22],[39],} \\
{[40],[91]}\end{array}$ \\
\hline & Stakeholders mgmt. & $\begin{array}{l}\text { Interactions and } \\
\text { cooperation with } \\
\text { internal and external }\end{array}$ & Action Research \\
\hline
\end{tabular}




\begin{tabular}{|c|c|c|c|}
\hline & & $\begin{array}{l}\text { stakeholders to increase } \\
\text { participation and create } \\
\text { awareness (within the } \\
\text { boundaries of the TT } \\
\text { process). }\end{array}$ & \\
\hline & $\begin{array}{l}\text { Senior mgmt. } \\
\text { involvement }\end{array}$ & $\begin{array}{l}\text { Awareness, approval, } \\
\text { support, and active } \\
\text { participation of senior } \\
\text { managers in the TT } \\
\text { process. }\end{array}$ & [19] \\
\hline \multirow[t]{3}{*}{ Organizational Culture } & $\begin{array}{l}\text { Communication and } \\
\text { knowledge mgmt. }\end{array}$ & $\begin{array}{c}\text { Relevance and } \\
\text { frequency of } \\
\text { interactions between } \\
\text { stakeholders, transfer } \\
\text { and sharing of } \\
\text { knowledge between } \\
\text { different departments } \\
\text { (outside the boundaries } \\
\text { of the TT process). }\end{array}$ & [37] \\
\hline & Funding & $\begin{array}{l}\text { Ease of access and level } \\
\text { of funding for transfer } \\
\text { activities. }\end{array}$ & [19], [59] \\
\hline & Absorptive capacity & $\begin{array}{l}\text { Capabilities related to } \\
\text { identifying valuable } \\
\text { information, assessing, } \\
\text { and using it. }\end{array}$ & {$[22]$} \\
\hline
\end{tabular}




\begin{tabular}{|c|c|c|c|}
\hline & Long-range planning & $\begin{array}{l}\text { Corporate strategy and } \\
\text { long-term technology } \\
\text { planning. }\end{array}$ & {$[2],[34]$} \\
\hline \multirow{4}{*}{ Technical } & TRL assessment & $\begin{array}{c}\text { Determination of } \\
\text { technology readiness } \\
\text { levels and related } \\
\text { metrics, e.g., IRL; SRL; } \\
\text { RD3. }\end{array}$ & Action Research \\
\hline & Data mgmt. & $\begin{array}{c}\text { Standardization, } \\
\text { acquisition, storage, } \\
\text { recovery, and analysis of } \\
\text { projects data. }\end{array}$ & Action Research \\
\hline & Technology valuation & $\begin{array}{l}\text { Technology assessment } \\
\text { studies at several } \\
\text { different points } \\
\text { throughout the } \\
\text { technology development } \\
\text { cycle. }\end{array}$ & [36] \\
\hline & Proposal assessment & $\begin{array}{l}\text { Qualitative and } \\
\text { quantitative } \\
\text { assessments of research } \\
\text { proposals. }\end{array}$ & [19] \\
\hline Process & Benefit mgmt. & $\begin{array}{l}\text { Identification, } \\
\text { understanding, } \\
\text { description, }\end{array}$ & [19] \\
\hline
\end{tabular}




\begin{tabular}{|c|c|c|c|}
\hline & & $\begin{array}{l}\text { classification, and } \\
\text { monitoring of benefits. }\end{array}$ & \\
\hline & Risk mgmt. & $\begin{array}{c}\text { Identification, } \\
\text { understanding, } \\
\text { description, } \\
\text { classification, and } \\
\text { monitoring of risks and } \\
\text { opportunities. }\end{array}$ & Action Research \\
\hline & $\begin{array}{l}\text { Outcomes and } \\
\text { decisions }\end{array}$ & $\begin{array}{l}\text { Discussions, reports and } \\
\text { decisions made out of } \\
\text { the TT process. }\end{array}$ & Action Research \\
\hline & $\begin{array}{l}\text { TT mechanisms } \\
\text { mgmt. }\end{array}$ & $\begin{array}{c}\text { Identification, } \\
\text { understanding, } \\
\text { description, } \\
\text { classification, and } \\
\text { monitoring of potential } \\
\text { TT mechanisms. }\end{array}$ & [1], [3], [22] \\
\hline Strategic Alignment & $\begin{array}{l}\text { Value, impact and } \\
\text { applicability mgmt. }\end{array}$ & $\begin{array}{l}\text { Economic value and } \\
\text { strategic fit; level of } \\
\text { impact of daily } \\
\text { operations and beyond; } \\
\text { Ease of applicability and } \\
\text { extra requirements, } \\
\text { such as adjustments, } \\
\text { adaptations, and special } \\
\text { training. }\end{array}$ & Action Research \\
\hline
\end{tabular}




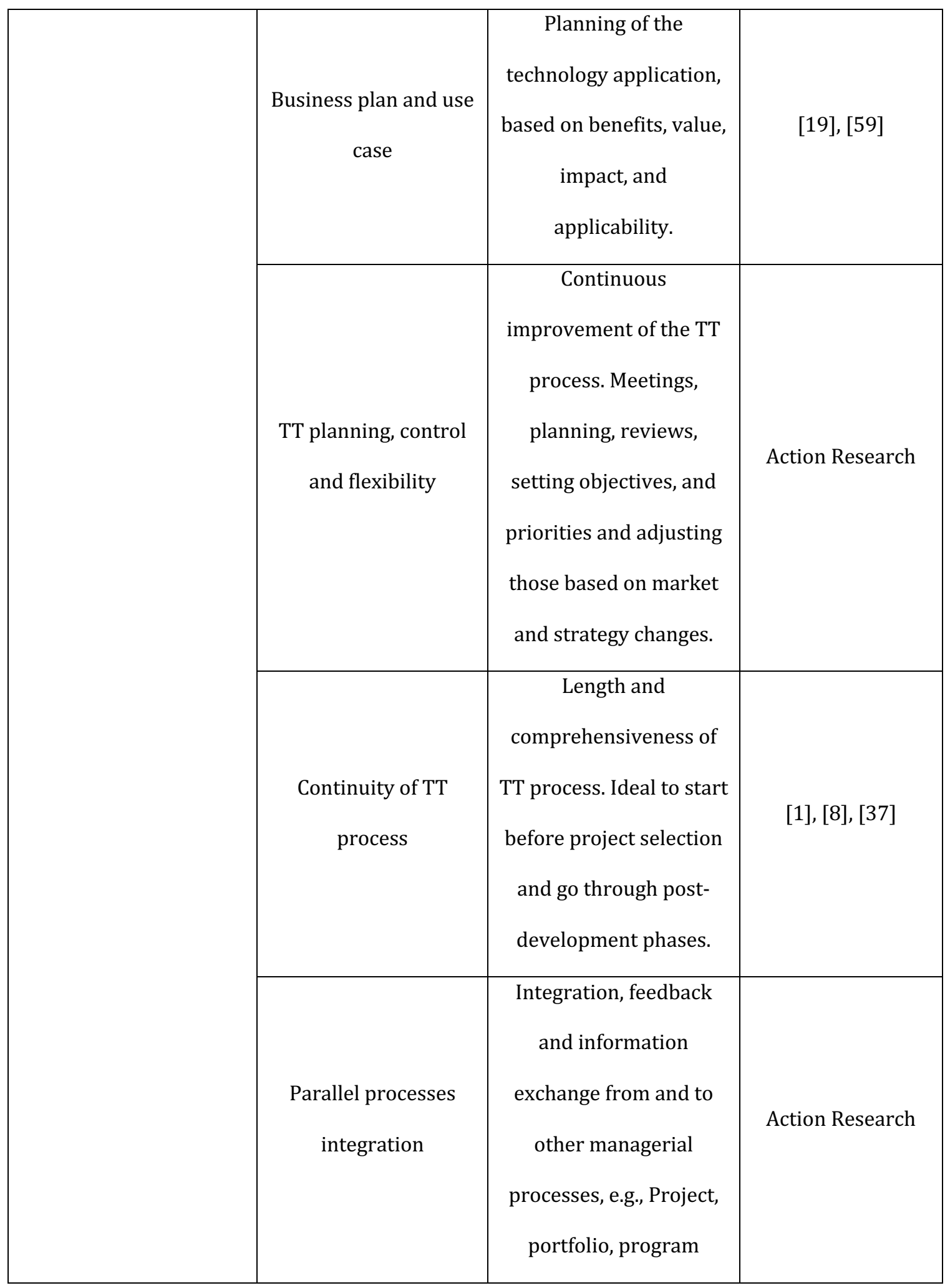




\begin{tabular}{|l|l|l|l|}
\hline & & $\begin{array}{c}\text { management, and } \\
\text { roadmapping. }\end{array}$ & \\
\hline
\end{tabular}

\section{Expert Panels Formation}

Experts were identified based on their skills and exposure to technology management and technology transfer concepts. This section brings an explanation of the rationale behind the process of choosing experts and building the panels.

When building the panels, the aim was to have between six and twelve experts per panel, following previous studies [19], [263], [267], [268], and also taking as precedent past studies using as little as three experts per panel [264], [266], while keeping in mind that the quality of experts is paramount and trumps the mere quantity. The basic criteria for choosing and inviting experts to participate in the study were: Expertise in the field, contributions to the field, absence of conflicts of interest, willingness to participate, availability to participate. The methods used in selecting the experts were social network analysis and professional connections, both complemented by snowball sampling.

The distribution of experts across the model was done in a way as to take advantage of each expert expertise and to avoid expert "overuse" in order to mitigate potential bias and logical inconsistencies, while maintaining their willingness and commitment towards the study. All experts were grouped into four categories: Professors and researchers, senior manager, middle managers, and project managers. These were used as a basis to build four expert profiles that were assigned to different 
sections of the model for validation and quantification purposes, according to the alignment between each model section and each expert profile.

The four expert profiles are described below:

- Profile A

- Senior managers + professors and researchers

- Profile B

- Senior managers + middle managers + professors and researchers

- Profile C

- Middle managers + project managers + professors and researchers

- Profile D

- Project managers + professors and researchers

Senior management-level personnel were assigned to sections of the model where a holistic view of the business needs is required. Project managers were assigned to parts of the model where knowledge and experience regarding the daily issues and details of $\mathrm{R} \& \mathrm{D}$ management and technology transfer are required. Medium-level managers were assigned to sections of the model where a tactical view is required, i.e., a mixture between strategic and operational perspectives. Finally, representatives from academia were used in all profiles in order to balance the points of view brought to the model validation and quantification with the assumption being that an experienced researcher in the field of technology transfer would be able to speak to all perspectives in the model. Figure 14 shows the assignment of expert profiles for different parts of the model. 
- Perspectives Level: Profile A

- Factors Level - HR and Stakeholders: Profile D

- $\quad$ Factors Level - Organizational Culture: Profile B

- Factors Level - Technical: Profile C

- Factors Level - Process: Profile C

- Factors Level - Strategic Alignment: Profile B.

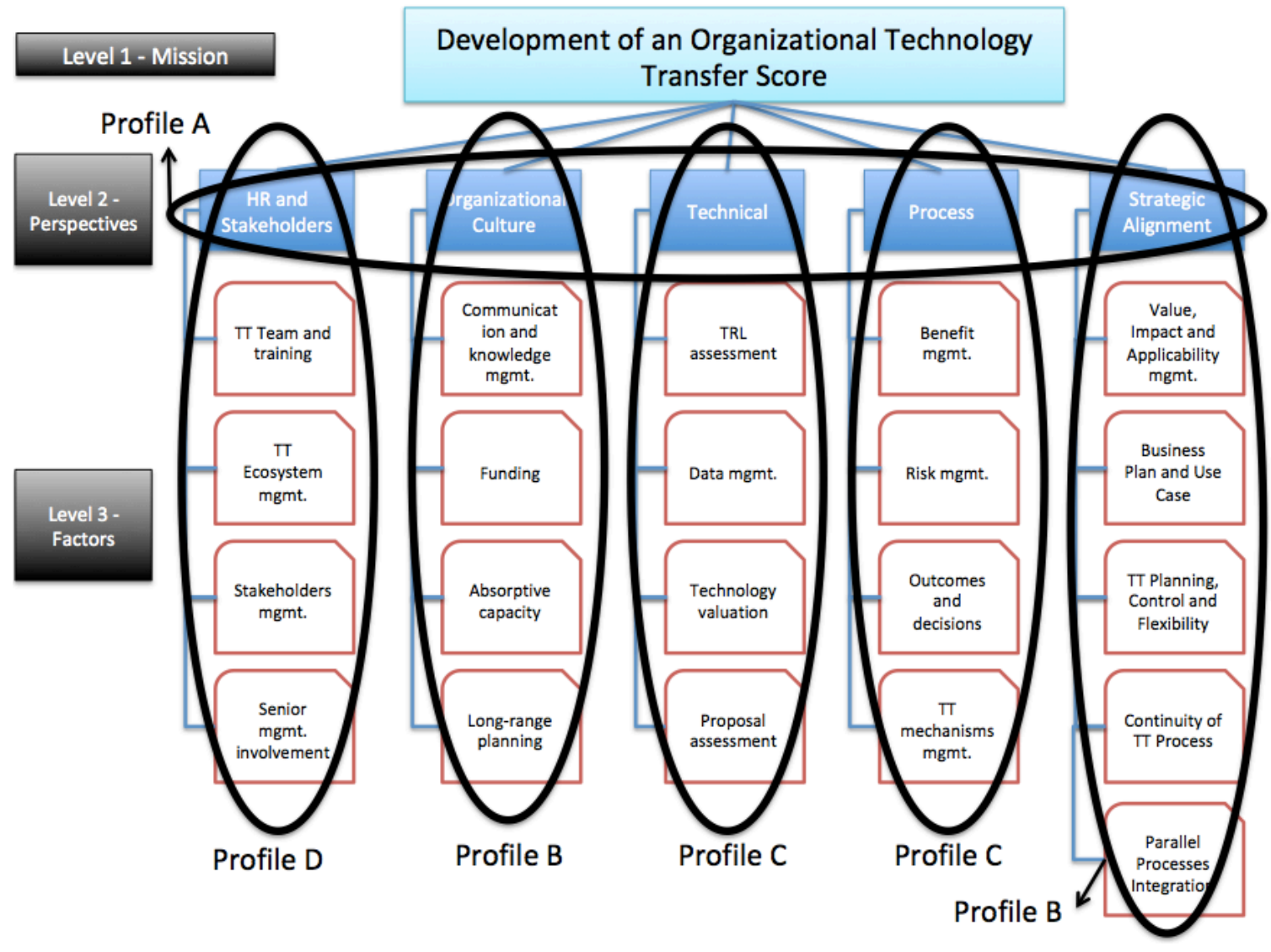

Figure 14 - Expert Panels Distribution

In total, thirty-nine experts participated in the study. Below are the expert distributions in terms of category, background and profiles since each expert can fit 
in more than one profile, the total number of experts in the four profiles amounts to more than 39:

- Categories
○ Senior managers: $12(30.7 \%)$
○ Middle managers: 12 (30.7\%)
○ Project managers: $4(10.3 \%)$
○ Professors / researchers: $11(28.2 \%)$

- Background
○ Industry: $15(38.5 \%)$
○ Government: 7 (17.9\%)
○ Academia: 17 (43.6\%)

- Profiles
○ A: 23
○ B: 35
○ C: 27
○ D: 15

Table 31 brings general information about the 39 experts.

Table 31 - List of Experts

\begin{tabular}{|c|c|c|c|}
\hline Expert & Title & Background & Category \\
\hline 1 & Program Manager & Industry & Middle manager \\
\hline 2 & Senior Research Scientist & Industry & Middle manager \\
\hline 3 & Program Manager & Industry & Middle manager \\
\hline 4 & Director (Retired) & Industry & Senior manager \\
\hline
\end{tabular}




\begin{tabular}{|c|c|c|c|}
\hline 5 & Program Manager & Government & Middle manager \\
\hline 6 & Managing Partner & Industry & Senior manager \\
\hline 7 & Professor & Academia & Researcher \\
\hline 8 & Technology Transfer Officer & Academia & Senior manager \\
\hline 9 & Professor & Academia & Researcher \\
\hline 10 & Professor & Academia & Researcher \\
\hline 11 & Planning Analyst & Industry & Project manager \\
\hline 12 & President & Industry & Senior manager \\
\hline 13 & Professor & Academia & Researcher \\
\hline 14 & Senior Consultant & Industry & Middle manager \\
\hline 15 & Professor & Academia & Researcher \\
\hline 16 & Systems Engineer & Government & Middle manager \\
\hline 17 & Professor & Academia & Researcher \\
\hline 18 & Professor & Academia & Researcher \\
\hline 19 & Professor & Academia & Researcher \\
\hline 20 & Director & Government & Senior manager \\
\hline 21 & Technology Transfer Manager & Government & Project manager \\
\hline 22 & Technology Transfer Director & Government & Senior manager \\
\hline 23 & Professor & Academia & Researcher \\
\hline 24 & Professor & Academia & Researcher \\
\hline 25 & Professor & Academia & Researcher \\
\hline 26 & R\&D Manager & Industry & Middle manager \\
\hline 27 & Project Manager & Industry & Project manager \\
\hline 28 & Director & Industry & Senior manager \\
\hline 29 & CEO & Industry & Senior manager \\
\hline 30 & Technology Transfer Director & Academia & Middle manager \\
\hline 31 & $\mathrm{COO}$ & Industry & Senior manager \\
\hline 32 & VP Commercialization & Academia & Senior manager \\
\hline 33 & Assistant Director & Academia & Middle manager \\
\hline 34 & Business Operations Director & Academia & Middle manager \\
\hline 35 & R\&D Manager & Industry & Middle manager \\
\hline 36 & Innovation Associate & Academia & Middle manager \\
\hline 37 & Senior Project Manager & Government & Project manager \\
\hline 38 & Director & Industry & Senior manager \\
\hline 39 & Technology Transfer Director & Government & Senior manager \\
\hline
\end{tabular}

Having recruited the experts and having in mind the rationale aforementioned, twelve expert panels were built. Panel 1 through 6 for model 
validation and Panels 7 through 12 for model quantification. The following are the lists of experts per panel (Table 32 to Table 43).

Table 32 - Panel 1

\begin{tabular}{|c|c|c|c|c|c|}
\hline Panel & Purpose & Expert & Title & Background & Category \\
\hline \multirow{4}{*}{1} & & 4 & Director (retired) & Industry & Senior manager \\
\cline { 3 - 6 } & & 6 & Managing Partner & Industry & Senior manager \\
\cline { 3 - 6 } & Validation & of \\
\cline { 3 - 6 } & Perspectives & 7 & Professor & Academia & Researcher \\
\cline { 3 - 6 } & & 13 & Professor & Academia & Researcher \\
\cline { 3 - 6 } & & Professor & Academia & Researcher \\
\cline { 3 - 6 } & & 20 & Director & Government & Senior manager \\
\cline { 3 - 6 } & & 24 & Professor & Academia & Researcher \\
\hline
\end{tabular}

Table 33 - Panel 2

\begin{tabular}{|c|c|c|c|c|c|}
\hline Panel & Purpose & Expert & Title & Background & Category \\
\hline & & 11 & Planning Analyst & Industry & $\begin{array}{c}\text { Project } \\
\text { manager }\end{array}$ \\
\cline { 3 - 6 } 2 & $\begin{array}{c}\text { Validation } \\
\text { of Factors } \\
\text { HR and }\end{array}$ & 20 & Director & Government & $\begin{array}{c}\text { Senior } \\
\text { manager }\end{array}$ \\
\cline { 3 - 6 } & $\begin{array}{c}\text { Stakeholder } \\
\text { S }\end{array}$ & 21 & $\begin{array}{c}\text { Technology } \\
\text { Transfer Manager }\end{array}$ & Government & $\begin{array}{c}\text { Project } \\
\text { manager }\end{array}$ \\
\cline { 3 - 6 } & & 39 & Project Manager & Industry & $\begin{array}{c}\text { Project } \\
\text { manager }\end{array}$ \\
\cline { 3 - 6 } & & TT Director & Government & $\begin{array}{c}\text { Senior } \\
\text { manager }\end{array}$ \\
\hline
\end{tabular}

Table 34 - Panel 3

\begin{tabular}{|c|c|c|c|c|c|}
\hline Panel & Purpose & Expert & Title & Background & Category \\
\hline & Validation of & 1 & $\begin{array}{c}\text { Program } \\
\text { Manager }\end{array}$ & Industry & $\begin{array}{c}\text { Middle } \\
\text { manager }\end{array}$ \\
\cline { 3 - 6 } 3 & $\begin{array}{c}\text { Factors - } \\
\text { Organizational } \\
\text { Culture }\end{array}$ & 2 & $\begin{array}{c}\text { Senior Research } \\
\text { Scientist }\end{array}$ & Industry & $\begin{array}{c}\text { Middle } \\
\text { manager }\end{array}$ \\
\cline { 3 - 6 } & 5 & $\begin{array}{c}\text { Program } \\
\text { Manager }\end{array}$ & Government & $\begin{array}{c}\text { Middle } \\
\text { manager }\end{array}$ \\
\hline
\end{tabular}




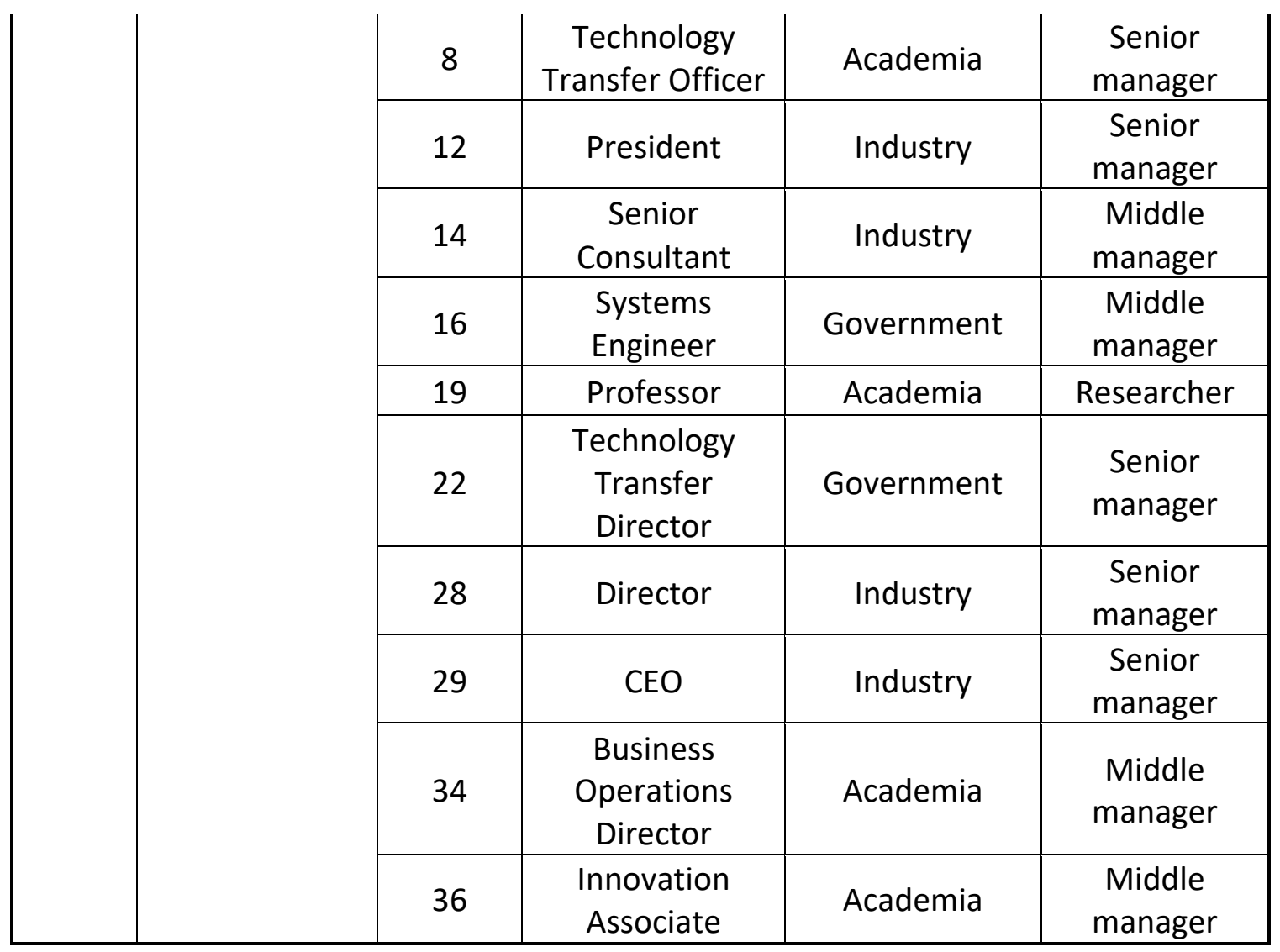

Table 35 - Panel 4

\begin{tabular}{|c|c|c|c|c|c|}
\hline Panel & Purpose & Expert & Title & Background & Category \\
\hline \multirow{10}{*}{4} & \multirow{10}{*}{$\begin{array}{l}\text { Validation } \\
\text { of Factors } \\
\text { - Technical }\end{array}$} & 3 & $\begin{array}{l}\text { Program } \\
\text { Manager }\end{array}$ & Industry & Middle manager \\
\hline & & 5 & $\begin{array}{l}\text { Program } \\
\text { Manager }\end{array}$ & Government & Middle manager \\
\hline & & 11 & Planning Analyst & Industry & Project manager \\
\hline & & 17 & Professor & Academia & Researcher \\
\hline & & 19 & Professor & Academia & Researcher \\
\hline & & 23 & Professor & Academia & Researcher \\
\hline & & 26 & R\&D Manager & Industry & Middle manager \\
\hline & & 27 & Manager & Industry & Project manager \\
\hline & & 33 & Assistant Director & Academia & Middle manager \\
\hline & & 37 & $\begin{array}{c}\text { Senior Project } \\
\text { Manager }\end{array}$ & Government & Project manager \\
\hline
\end{tabular}


Table 36 - Panel 5

\begin{tabular}{|c|c|c|c|c|c|}
\hline Panel & Purpose & Expert & Title & Background & Category \\
\hline \multirow{8}{*}{5} & \multirow{8}{*}{$\begin{array}{l}\text { Validation } \\
\text { of Factors } \\
\text { - Process }\end{array}$} & 1 & Program Manager & Industry & Middle manager \\
\hline & & 3 & Program Manager & Industry & Middle manager \\
\hline & & 7 & Professor & Academia & Researcher \\
\hline & & 13 & Professor & Academia & Researcher \\
\hline & & 14 & Senior Consultant & Industry & Middle manager \\
\hline & & 21 & Technology Transfer Manager & Government & Project manager \\
\hline & & 34 & Business Operations Director & Academia & Middle manager \\
\hline & & 36 & Innovation Associate & Academia & Middle manager \\
\hline
\end{tabular}

Table 37 - Panel 6

\begin{tabular}{|c|c|c|c|c|c|}
\hline Panel & Purpose & Expert & Title & Background & Category \\
\hline \multirow{11}{*}{6} & \multirow{11}{*}{$\begin{array}{l}\text { Validation } \\
\text { of Factors } \\
\text { - Strategic } \\
\text { Alignment }\end{array}$} & 2 & Senior Research Scientist & Industry & Middle manager \\
\hline & & 4 & Director (retired) & Industry & Senior manager \\
\hline & & 6 & Managing Partner & Industry & Senior manager \\
\hline & & 8 & Technology Transfer Officer & Academia & Senior manager \\
\hline & & 16 & Systems Engineer & Government & Middle manager \\
\hline & & 22 & Technology Transfer Director & Government & Senior manager \\
\hline & & 28 & \begin{tabular}{|c|} 
Director \\
\end{tabular} & Industry & Senior manager \\
\hline & & 29 & CEO & Industry & Senior manager \\
\hline & & 31 & $\mathrm{COO}$ & Industry & Senior manager \\
\hline & & 32 & VP of Commercialization & Academia & Senior manager \\
\hline & & 39 & Technology Transfer Director & Government & Senior manager \\
\hline
\end{tabular}

Table 38 - Panel 7

\begin{tabular}{|c|c|c|c|c|c|}
\hline Panel & Purpose & Expert & Title & Background & Category \\
\hline \multirow{4}{*}{7} & \multirow{2}{*}{$\begin{array}{c}\text { Quantification } \\
\text { of }\end{array}$} & 6 & $\begin{array}{c}\text { Managing } \\
\text { Partner }\end{array}$ & Industry & $\begin{array}{c}\text { Senior } \\
\text { manager }\end{array}$ \\
\cline { 3 - 6 } & Perspectives & 10 & Professor & Academia & Researcher \\
\cline { 3 - 6 } & & 20 & Professor & Academia & Researcher \\
\cline { 3 - 6 } & & Director & Government & $\begin{array}{c}\text { Senior } \\
\text { manager }\end{array}$ \\
\hline
\end{tabular}




\begin{tabular}{|l|l|l|l|l|l|} 
& 31 & COO & Industry & $\begin{array}{c}\text { Senior } \\
\text { manager }\end{array}$ \\
\hline
\end{tabular}

Table 39 - Panel 8

\begin{tabular}{|c|c|c|c|c|c|}
\hline Panel & Purpose & Expert & Title & Background & Category \\
\cline { 3 - 6 } & 17 & Professor & Academia & Researcher \\
\cline { 3 - 6 } & \begin{tabular}{c} 
Quantification \\
of Factors - HR \\
and \\
\cline { 3 - 6 }
\end{tabular} & 21 & Director & Government & $\begin{array}{c}\text { Senior } \\
\text { manager }\end{array}$ \\
\cline { 3 - 6 } & \begin{tabular}{c} 
Stakeholders \\
\cline { 3 - 6 }
\end{tabular} & 23 & $\begin{array}{c}\text { Technology } \\
\text { Mansfer }\end{array}$ & Government & $\begin{array}{c}\text { Project } \\
\text { manager }\end{array}$ \\
\cline { 3 - 6 } & 37 & $\begin{array}{c}\text { Senior Project } \\
\text { Manager }\end{array}$ & Government & $\begin{array}{c}\text { Project } \\
\text { manager }\end{array}$ \\
\hline
\end{tabular}

Table 40 - Panel 9

\begin{tabular}{|c|c|c|c|c|c|}
\hline Panel & Purpose & Expert & Title & Background & Category \\
\hline \multirow{8}{*}{9} & \multirow{8}{*}{$\begin{array}{l}\text { Quantification } \\
\text { of Factors - } \\
\text { Organizational } \\
\text { Culture }\end{array}$} & 1 & Program Manager & Industry & $\begin{array}{c}\text { Middle } \\
\text { manager }\end{array}$ \\
\hline & & 8 & $\begin{array}{c}\text { Technology Transfer } \\
\text { Officer }\end{array}$ & Academia & $\begin{array}{c}\text { Senior } \\
\text { manager }\end{array}$ \\
\hline & & 14 & Senior Consultant & Industry & $\begin{array}{c}\text { Middle } \\
\text { manager }\end{array}$ \\
\hline & & 16 & Systems Engineer & Government & $\begin{array}{c}\text { Middle } \\
\text { manager }\end{array}$ \\
\hline & & 22 & $\begin{array}{c}\text { Technology Transfer } \\
\text { Director }\end{array}$ & Government & $\begin{array}{c}\text { Senior } \\
\text { manager }\end{array}$ \\
\hline & & 28 & Director & Industry & $\begin{array}{c}\text { Senior } \\
\text { manager }\end{array}$ \\
\hline & & 32 & VP of Commercialization & Academia & $\begin{array}{l}\text { Senior } \\
\text { manager }\end{array}$ \\
\hline & & 36 & Innovation Associate & Academia & $\begin{array}{c}\text { Middle } \\
\text { manager }\end{array}$ \\
\hline
\end{tabular}

Table 41 - Panel 10

\begin{tabular}{|l|l|l|l|l|l|}
\hline Panel & Purpose & Expert & Title & Background & Category \\
\hline
\end{tabular}




\begin{tabular}{|c|c|c|c|c|c|}
\hline \multirow{5}{*}{10} & \multirow{5}{*}{$\begin{array}{l}\text { Quantification } \\
\text { of Factors - } \\
\text { Technical }\end{array}$} & 3 & $\begin{array}{l}\text { Program } \\
\text { Manager }\end{array}$ & Industry & $\begin{array}{c}\text { Middle } \\
\text { manager }\end{array}$ \\
\hline & & 5 & $\begin{array}{l}\text { Program } \\
\text { Manager }\end{array}$ & Government & $\begin{array}{c}\text { Middle } \\
\text { manager }\end{array}$ \\
\hline & & 17 & Professor & Academia & Researcher \\
\hline & & 26 & $\begin{array}{c}\text { R\&D } \\
\text { Manager }\end{array}$ & Industry & $\begin{array}{c}\text { Middle } \\
\text { manager }\end{array}$ \\
\hline & & 37 & $\begin{array}{l}\text { Senior } \\
\text { Project } \\
\text { Manager }\end{array}$ & Government & $\begin{array}{l}\text { Project } \\
\text { manager }\end{array}$ \\
\hline
\end{tabular}

Table 42 - Panel 11

\begin{tabular}{|c|c|c|c|c|c|}
\hline Panel & Purpose & Expert & Title & Background & Category \\
\hline \multirow{7}{*}{11} & \multirow{7}{*}{$\begin{array}{c}\text { Quantification } \\
\text { of Factors - } \\
\text { Process }\end{array}$} & 1 & $\begin{array}{l}\text { Program } \\
\text { Manager }\end{array}$ & Industry & $\begin{array}{l}\text { Middle } \\
\text { manager }\end{array}$ \\
\hline & & 7 & Professor & Academia & Researcher \\
\hline & & 13 & Professor & Academia & Researcher \\
\hline & & 14 & $\begin{array}{c}\text { Senior } \\
\text { Consultant }\end{array}$ & Industry & $\begin{array}{c}\text { Middle } \\
\text { manager }\end{array}$ \\
\hline & & 21 & $\begin{array}{c}\text { Technology } \\
\text { Transfer } \\
\text { Manager }\end{array}$ & Government & $\begin{array}{l}\text { Project } \\
\text { manager }\end{array}$ \\
\hline & & 26 & R\&D Manager & Industry & $\begin{array}{l}\text { Middle } \\
\text { manager }\end{array}$ \\
\hline & & 36 & $\begin{array}{l}\text { Innovation } \\
\text { Associate }\end{array}$ & Academia & $\begin{array}{l}\text { Middle } \\
\text { manager }\end{array}$ \\
\hline
\end{tabular}

Table 43 - Panel 12

\begin{tabular}{|c|c|c|c|c|c|}
\hline Panel & Purpose & Expert & Title & Background & Category \\
\hline \multirow{5}{*}{12} & \multirow{5}{*}{$\begin{array}{c}\text { Quantification } \\
\text { of Factors - } \\
\text { Strategic } \\
\text { Alignment }\end{array}$} & 6 & $\begin{array}{c}\text { Managing } \\
\text { Partner }\end{array}$ & Industry & $\begin{array}{c}\text { Senior } \\
\text { manager }\end{array}$ \\
\hline & & 8 & $\begin{array}{c}\text { Technology } \\
\text { Transfer Officer }\end{array}$ & Academia & $\begin{array}{c}\text { Senior } \\
\text { manager }\end{array}$ \\
\hline & & 16 & $\begin{array}{l}\text { Systems } \\
\text { Engineer }\end{array}$ & Government & $\begin{array}{l}\text { Middle } \\
\text { manager }\end{array}$ \\
\hline & & 22 & $\begin{array}{c}\text { Technology } \\
\text { Transfer Director }\end{array}$ & Government & $\begin{array}{l}\text { Senior } \\
\text { manager }\end{array}$ \\
\hline & & 24 & Professor & Academia & Researcher \\
\hline
\end{tabular}




\begin{tabular}{|c|c|c|c|c|c|} 
& 28 & Director & Industry & $\begin{array}{c}\text { Senior } \\
\text { manager }\end{array}$ \\
\cline { 3 - 5 } & 31 & COO & Industry & $\begin{array}{c}\text { Senior } \\
\text { manager }\end{array}$ \\
\cline { 3 - 5 } & 39 & $\begin{array}{c}\text { Technology } \\
\text { Transfer Director }\end{array}$ & Government & $\begin{array}{c}\text { Senior } \\
\text { manager }\end{array}$ \\
\hline
\end{tabular}

Regarding the formation of the above listed panels, there are two details to be explained:

- The difference between the total number of experts per profile and number of experts per panel:

- Example: Profile A has 23 experts but Panel 1 has only 7 experts

- Reasoning: In order to avoid expert overuse, to mitigate bias and logical inconsistency, and to maintain an acceptable level of commitment and motivation towards the study.

- The difference between the number of experts in the validation panels 1-6 and the number of experts in the quantification panels 7-12:

○ Example: Panel 1 has seven experts, but Panel 7 has only five.

- Reasoning: For several reasons some experts did not participate in all phases of the study, e.g., too much workload, health issues, family / professional issues, etc... 


\section{Model Validation}

\section{Perspectives Validation}

In order to validate the perspectives, a minimum of three-quarters $(75 \%)$ acceptance rate was used, i.e. if less than $75 \%$ of the experts agreed on a certain perspective, it would be removed from the model [276]. The survey instrument was sent to all experts in the panel, and $100 \%$ of them accepted the proposed perspectives, as shown in Table 44. As a result, all five original perspectives were kept. Some new items were suggested for the perspectives level. However, after deliberating with the experts that made the suggestions, it was determined that all suggested items were already being accounted for in the model, either in the factors level or using a different nomenclature, therefore no new item was added to the perspectives.

Table 44 - Perspectives Validation Results

\begin{tabular}{|c|c|c|c|c|c|}
\hline \multirow{2}{*}{ Panel } & Perspective & $\begin{array}{c}\text { Number of } \\
\text { experts that } \\
\text { agreed }\end{array}$ & $\begin{array}{c}\text { Number of } \\
\text { experts that } \\
\text { disagreed }\end{array}$ & $\begin{array}{c}\text { Total number } \\
\text { of experts }\end{array}$ & $\begin{array}{c}\% \text { of } \\
\text { YES }\end{array}$ \\
\hline \multirow{3}{*}{1} & HR and Stakeholders & 7 & 0 & 7 & $100 \%$ \\
\cline { 2 - 6 } & Organizational Culture & 7 & 0 & 7 & $100 \%$ \\
\cline { 2 - 6 } & Technical & 7 & 0 & 7 & $100 \%$ \\
\cline { 2 - 6 } & Process & 7 & 0 & 7 & $100 \%$ \\
\cline { 2 - 6 } & Strategic Alignment & 7 & 0 & 7 & $100 \%$ \\
\hline
\end{tabular}




\section{Factors Validation}

In order to validate the factors under each perspective, a minimum of threequarters (75\%) acceptance rate was used, i.e. if less than $75 \%$ of the experts agreed on a certain factor, it would be removed from the model. The survey instrument was sent to all experts in the respective panels, and the results are shown in Tables 45 through 49 .

Table 45 - Factors Validation Results for "HR and Stakeholders"

\begin{tabular}{|c|c|c|c|c|c|c|}
\hline \multirow{2}{*}{ Panel } & Perspective & Factor & $\begin{array}{c}\text { Number } \\
\text { of } \\
\text { experts } \\
\text { that } \\
\text { agreed }\end{array}$ & $\begin{array}{c}\text { Number of } \\
\text { experts } \\
\text { that } \\
\text { disagreed }\end{array}$ & $\begin{array}{c}\text { Total } \\
\text { number } \\
\text { of } \\
\text { experts }\end{array}$ & $\begin{array}{c}\text { \% of } \\
\text { YES }\end{array}$ \\
\hline \multirow{2}{*}{2} & \multirow{2}{*}{ HR and Stakeholders } & TT team and training & 6 & 0 & 6 & $\mathbf{1 0 0 \%}$ \\
\cline { 3 - 7 } & & TT ecosystem mgmt. & 5 & 1 & 6 & $\mathbf{8 3 . 3 \%}$ \\
\cline { 3 - 7 } & & Stakeholders mgmt. & 6 & 0 & 6 & $\mathbf{1 0 0 \%}$ \\
\cline { 3 - 7 } & & Senior mgmt. & 5 & 5 & 6 & $\mathbf{8 3 . 3 \%}$ \\
\hline
\end{tabular}

Table 46 - Factors Validation Results for "Organizational Culture"

\begin{tabular}{|c|c|c|c|c|c|c|}
\hline Panel & Perspective & Factor & $\begin{array}{c}\text { Number } \\
\text { of } \\
\text { experts } \\
\text { that } \\
\text { agreed }\end{array}$ & $\begin{array}{c}\text { Number of } \\
\text { experts } \\
\text { that } \\
\text { disagreed }\end{array}$ & $\begin{array}{c}\text { Total } \\
\text { number } \\
\text { of } \\
\text { experts }\end{array}$ & $\begin{array}{l}\% \text { of } \\
\text { YES }\end{array}$ \\
\hline \multirow{4}{*}{3} & \multirow{4}{*}{$\begin{array}{l}\text { Organizational } \\
\text { Culture }\end{array}$} & $\begin{array}{l}\text { Communication and } \\
\text { knowledge mgmt. }\end{array}$ & 12 & 1 & 13 & $92.3 \%$ \\
\hline & & Funding & 12 & 1 & 13 & $92.3 \%$ \\
\hline & & Absorptive capacity & 12 & 1 & 13 & $92.3 \%$ \\
\hline & & Long-range planning & 12 & 1 & 13 & $92.3 \%$ \\
\hline
\end{tabular}


Table 47 - Factors Validation Results for "Technical”

\begin{tabular}{|c|c|c|c|c|c|c|}
\hline \multirow{2}{*}{ Panel } & Perspective & Factor & $\begin{array}{c}\text { Number } \\
\text { of } \\
\text { experts } \\
\text { that } \\
\text { agreed }\end{array}$ & $\begin{array}{c}\text { Number of } \\
\text { experts } \\
\text { that } \\
\text { disagreed }\end{array}$ & $\begin{array}{c}\text { Total } \\
\text { number } \\
\text { of } \\
\text { experts }\end{array}$ & $\begin{array}{c}\text { \% of } \\
\text { YES }\end{array}$ \\
\hline \multirow{3}{*}{4} & \multirow{3}{*}{ Technical } & TRL assessment & 10 & 0 & 10 & $\mathbf{1 0 0 \%}$ \\
\cline { 3 - 8 } & & Data mgmt. & 7 & 3 & 10 & $\mathbf{7 0 \%}$ \\
\cline { 3 - 8 } & & Technology valuation & 10 & 0 & 10 & $\mathbf{1 0 0 \%}$ \\
\cline { 3 - 8 } & & Proposal assessment & 8 & 2 & 10 & $\mathbf{8 0 \%}$ \\
\hline
\end{tabular}

Table 48 - Factors Validation Results for "Process"

\begin{tabular}{|c|c|c|c|c|c|c|}
\hline Panel & Perspective & Factor & $\begin{array}{c}\text { Number } \\
\text { of } \\
\text { experts } \\
\text { that } \\
\text { agreed }\end{array}$ & $\begin{array}{l}\text { Number of } \\
\text { experts } \\
\text { that } \\
\text { disagreed }\end{array}$ & $\begin{array}{c}\text { Total } \\
\text { number } \\
\text { of } \\
\text { experts }\end{array}$ & $\begin{array}{l}\% \text { of } \\
\text { YES }\end{array}$ \\
\hline \multirow{4}{*}{5} & \multirow{4}{*}{ Process } & Benefit mgmt. & 8 & 0 & 8 & $100 \%$ \\
\hline & & Risk mgmt. & 8 & 0 & 8 & $100 \%$ \\
\hline & & $\begin{array}{l}\text { Outcomes and } \\
\text { decisions }\end{array}$ & 8 & 0 & 8 & $100 \%$ \\
\hline & & TT mechanisms mgmt. & 8 & 0 & 8 & $100 \%$ \\
\hline
\end{tabular}

Table 49 - Factors Validation Results for "Strategic Alignment"

\begin{tabular}{|c|c|c|c|c|c|c|}
\hline Panel & Perspective & Factor & $\begin{array}{c}\text { Number } \\
\text { of } \\
\text { experts } \\
\text { that } \\
\text { agreed }\end{array}$ & $\begin{array}{c}\text { Number of } \\
\text { experts } \\
\text { that } \\
\text { disagreed }\end{array}$ & $\begin{array}{c}\text { Total } \\
\text { number } \\
\text { of } \\
\text { experts }\end{array}$ & $\begin{array}{c}\text { \% of } \\
\text { YES }\end{array}$ \\
\hline 6 & \multirow{2}{*}{\begin{tabular}{c} 
Strategic Alignment \\
\cline { 3 - 7 }
\end{tabular}} & $\begin{array}{c}\text { Value, impact and } \\
\text { applicability mgmt. }\end{array}$ & 10 & 1 & 11 & $\mathbf{9 0 . 9 \%}$ \\
\cline { 3 - 7 } & $\begin{array}{c}\text { Business plan and use } \\
\text { case }\end{array}$ & 10 & 1 & 11 & $\mathbf{9 0 . 9 \%}$ \\
\hline
\end{tabular}




\begin{tabular}{|c|c|c|c|c|c|}
\hline \multirow{n}{*}{} & $\begin{array}{c}\text { TT planning, control } \\
\text { and flexibility }\end{array}$ & 11 & 0 & 11 & $\mathbf{1 0 0 \%}$ \\
\cline { 2 - 6 } & $\begin{array}{c}\text { Continuity of TT } \\
\text { process }\end{array}$ & 10 & 1 & 11 & $\mathbf{9 0 . 9 \%}$ \\
\cline { 2 - 6 } & $\begin{array}{c}\text { Parallel processes } \\
\text { integration }\end{array}$ & 11 & 0 & 11 & $\mathbf{1 0 0 \%}$ \\
\hline
\end{tabular}

As seen in the tables above, all factors had an acceptance rate higher than the 75\% threshold, except for "data management" under the "technical" perspective, which was removed from the model. Additionally, several new items were suggested under each perspective, but similarly to what happened in the perspectives validation, the vast majority of suggestions were already being accounted for in the model, e.g., in another part of the model, using a different nomenclature. In some cases, the suggestions were determined to be outside the scope of the study or too general to be considered. For every suggestion made, a discussion took place with the expert that made the suggestion in order to determine if the item should or should not have been added to the model.

Of all suggestions made, two were determined to be suitable for the model. Firstly, under the "HR and Stakeholder" perspective, the definition of the "Senior Management Involvement" factor, now includes the concept of technology transfer champions. Those would be senior management representatives, assigned to champion technology transfer activities throughout the organization and removing any potential roadblocks. This change will impact the levels of desirability of this factor, to be presented in following sections. Secondly, the factor "Innovative Culture" was added to the "Organizational Culture" perspective. This factor tries to capture and measure the innovation proneness of the organization, i.e., how accustomed to 
changes in technology and business models/processes the organization is, granted that the more accustomed to these changes, the easier and faster a technology transfer process would unfold in that organization. Below are the definitions of the two factors affected by these changes: .

- Senior management involvement: Awareness, approval, support, and active participation of senior managers in the TT process, including the indication of TT champions.

- Innovative Culture: The organization's ability and openness to implement innovation and changes regarding its business practices.

\section{Validated Model}

Figure 15 shows the validated model. 


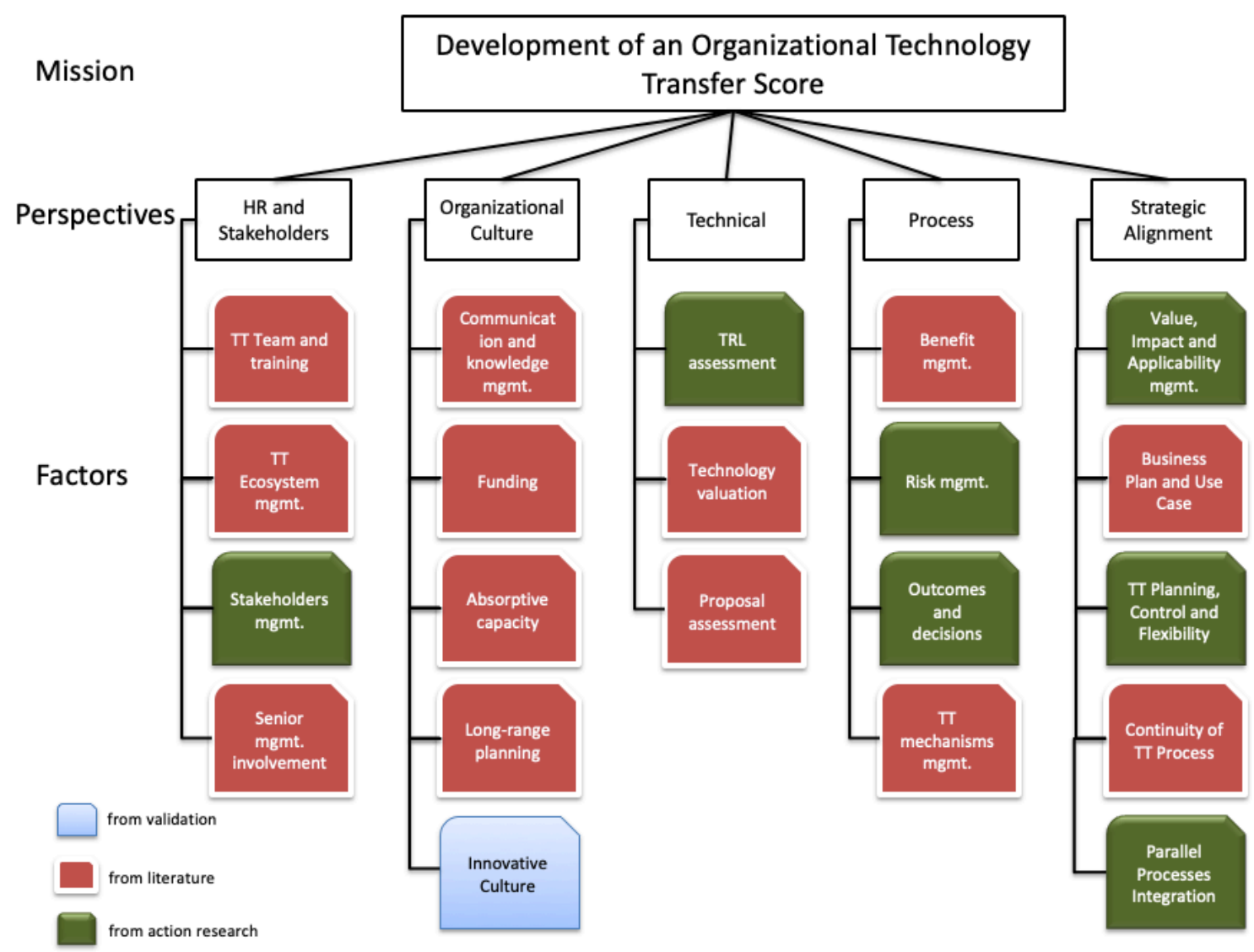

Figure 15 - Validated HDM Model

Figure 16 shows both versions of the model. It shows the before and after validation process, and highlights the differences, i.e., the removal of "Data management" from the "Technical" perspective and the addition of "Innovative Culture" into the "Organizational Culture" perspective. 


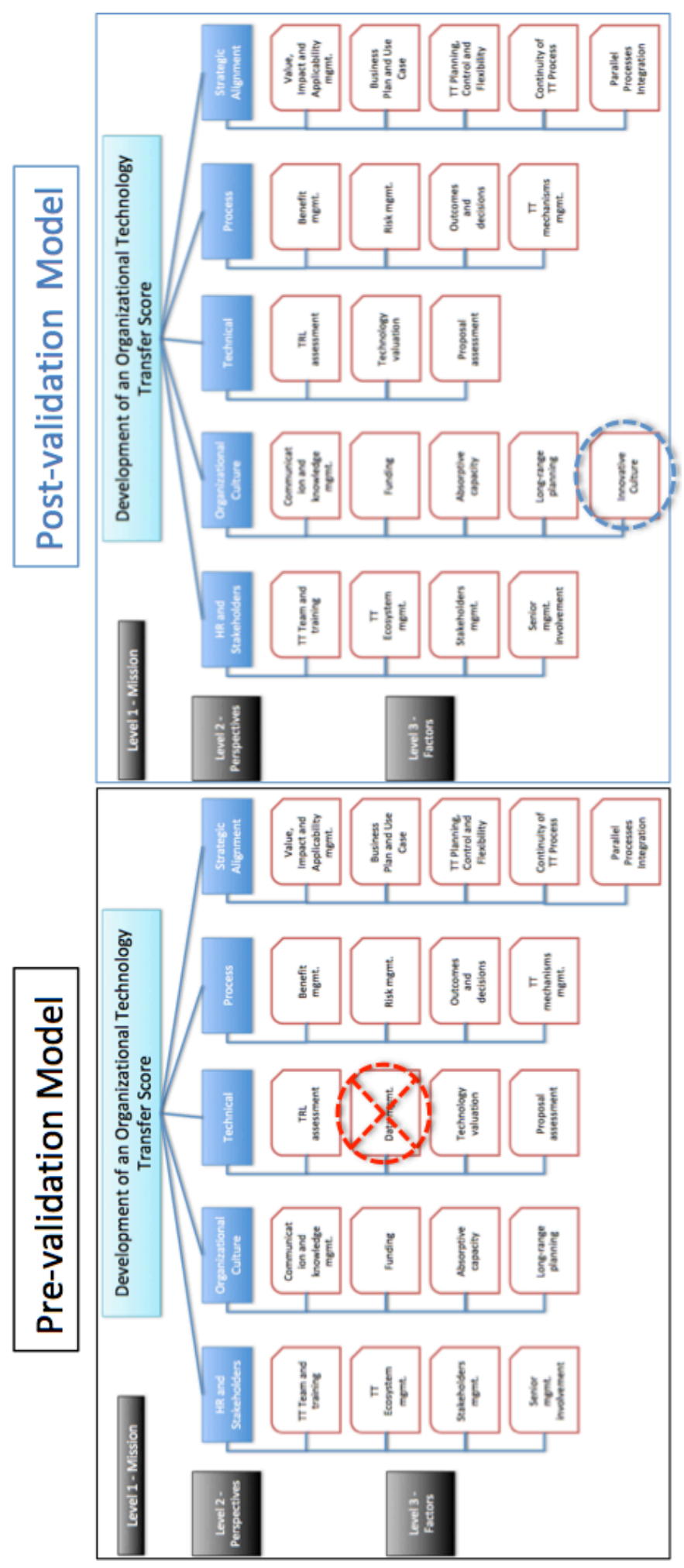

Figure 16 - Pre-validation Model vs. Post-Validation Model 


\section{Model Quantification}

Having validated the model, the next step is to quantify each perspective and factor through experts' judgments.

The experts' judgments were captured through pair-wise comparisons utilizing the constant-sum method, where each comparison is made by distributing 100 points between the pair of items being compared. The importance of each perspective relative to the determination of the TT score is obtained by the mathematical procedures explained earlier. The arithmetical mean of the judgments is used as a final result. Data is collected through an online survey tool and the analysis is done using the $\mathrm{HDM}^{\circledR}$ software, which also calculated the individual logical inconsistencies and the group disagreements. As a general rule, no inconsistency or disagreement higher than $10 \%$ would be accepted. Whenever the inconsistency and disagreement levels are below the $10 \%$ mark, no further action is taken. Conversely, when the levels are above the $10 \%$ threshold, extra steps are taken to further understand and analyze the impact of each particular situation towards the final results.

\section{Perspectives Quantification}

The following are the judgments for each expert and the average for each perspective, followed by an illustrative chart. 
Table 50 - Perspectives Quantification Results

\begin{tabular}{|c|c|c|c|c|c|c|c|}
\hline Panel & Expert & $\begin{array}{c}\text { HR and } \\
\text { Stakeholders }\end{array}$ & $\begin{array}{c}\text { Organizational } \\
\text { Culture }\end{array}$ & Technical & Process & $\begin{array}{c}\text { Strategic } \\
\text { Alignment }\end{array}$ & Inconsistency \\
\hline \multirow{7}{*}{7} & 13 & 0.23 & 0.15 & 0.15 & 0.23 & 0.23 & 0 \\
\hline & 10 & 0.40 & 0.18 & 0.08 & 0.08 & 0.26 & 0.05 \\
\hline & 31 & 0.18 & 0.16 & 0.23 & 0.14 & 0.29 & 0 \\
\hline & 20 & 0.18 & 0.18 & 0.22 & 0.15 & 0.27 & 0.01 \\
\hline & 6 & 0.14 & 0.25 & 0.19 & 0.25 & 0.17 & 0.01 \\
\hline & Mean & 0.23 & 0.18 & 0.17 & 0.17 & 0.24 & \\
\hline & & & & & \multicolumn{2}{|c|}{ Disagreement } & 0.055 \\
\hline
\end{tabular}

\section{Relative Importance of Perspectives}

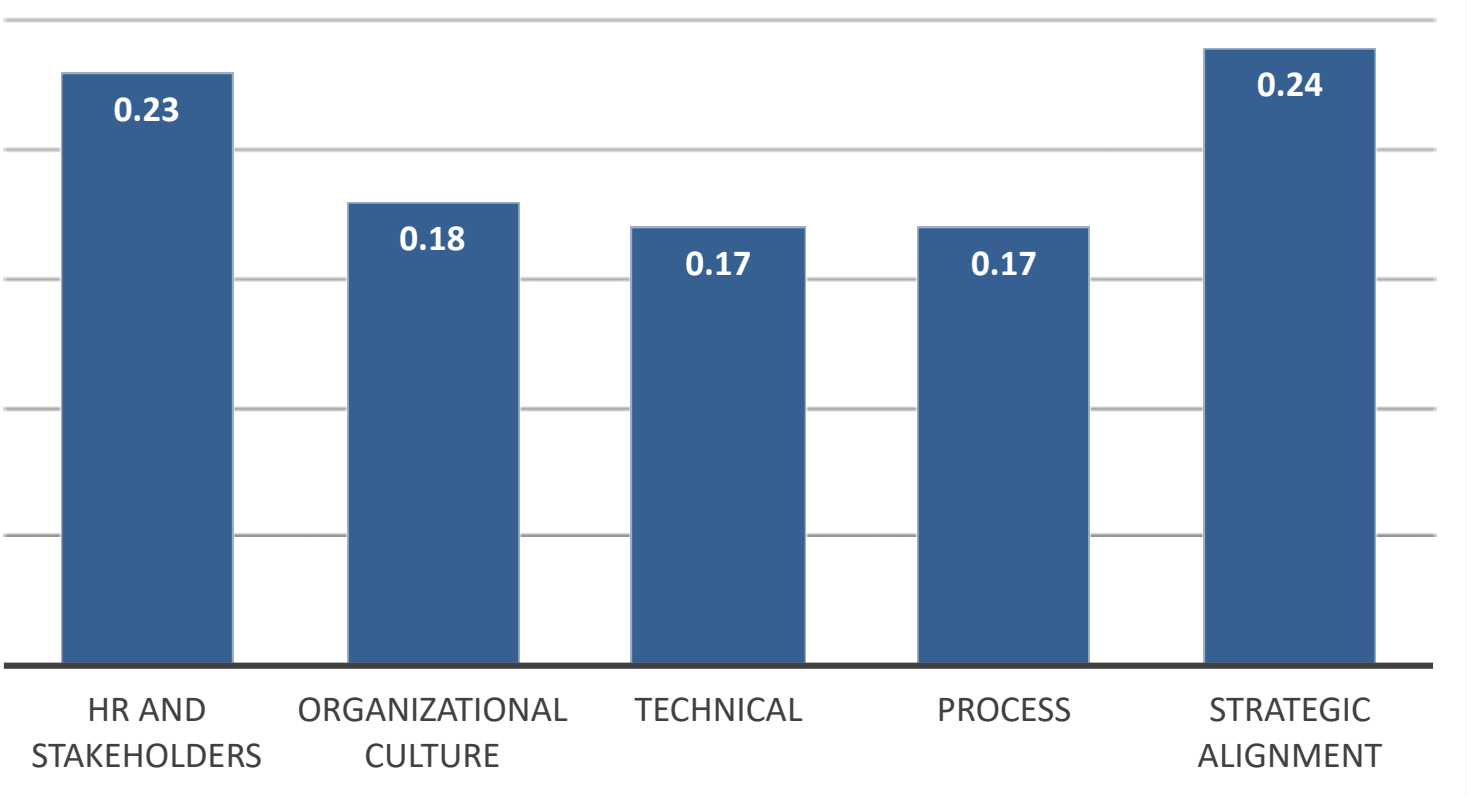

Figure 17 - Perspectives Quantification Chart

As shown in the table and graph above, "Strategic Alignment" is ranked as the most important perspective, contributing $24 \%$ towards the mission (technology transfer score), closely followed by "HR and Stakeholders", with 23\%. The other three perspectives have very similar rankings, with $18 \%$ being given to "Organizational 
Culture", and $17 \%$ to each of the remaining ones, "Process" and "Technical. The inconsistency and disagreement levels for Panel 7 are within the acceptable range.

\section{Factors Quantification}

The following are the quantification results for each of the twenty-one factors under each of the five perspectives. The judgments for every expert and the average for each factor are shown, followed by illustrative charts.

Table 51 - HR and Stakeholders Factors Quantification Results

\begin{tabular}{|c|c|c|c|c|c|c|}
\hline Panel & Expert & $\begin{array}{c}\text { TT team and } \\
\text { training }\end{array}$ & $\begin{array}{c}\text { TT ecosystem } \\
\text { mgmt }\end{array}$ & $\begin{array}{c}\text { Stakeholders } \\
\text { mgmt }\end{array}$ & $\begin{array}{c}\text { Senior mgmt } \\
\text { involvement }\end{array}$ & Inconsistency \\
\hline \multirow{6}{*}{8} & 23 & 0.31 & 0.28 & 0.21 & 0.20 & 0.01 \\
\cline { 2 - 7 } & 17 & 0.23 & 0.28 & 0.25 & 0.25 & 0 \\
\cline { 2 - 7 } & 21 & 0.38 & 0.08 & 0.27 & 0.27 & 0.01 \\
\cline { 2 - 7 } & 37 & 0.48 & 0.19 & 0.29 & 0.04 & 0.04 \\
\cline { 2 - 7 } & 20 & 0.24 & 0.36 & 0.24 & 0.16 & 0 \\
\cline { 2 - 7 } & Mean & $\mathbf{0 . 3 3}$ & $\mathbf{0 . 2 4}$ & $\mathbf{0 . 2 5}$ & $\mathbf{0 . 1 8}$ & \\
\cline { 2 - 7 } & \multicolumn{7}{|r|}{} \\
\cline { 2 - 7 }
\end{tabular}




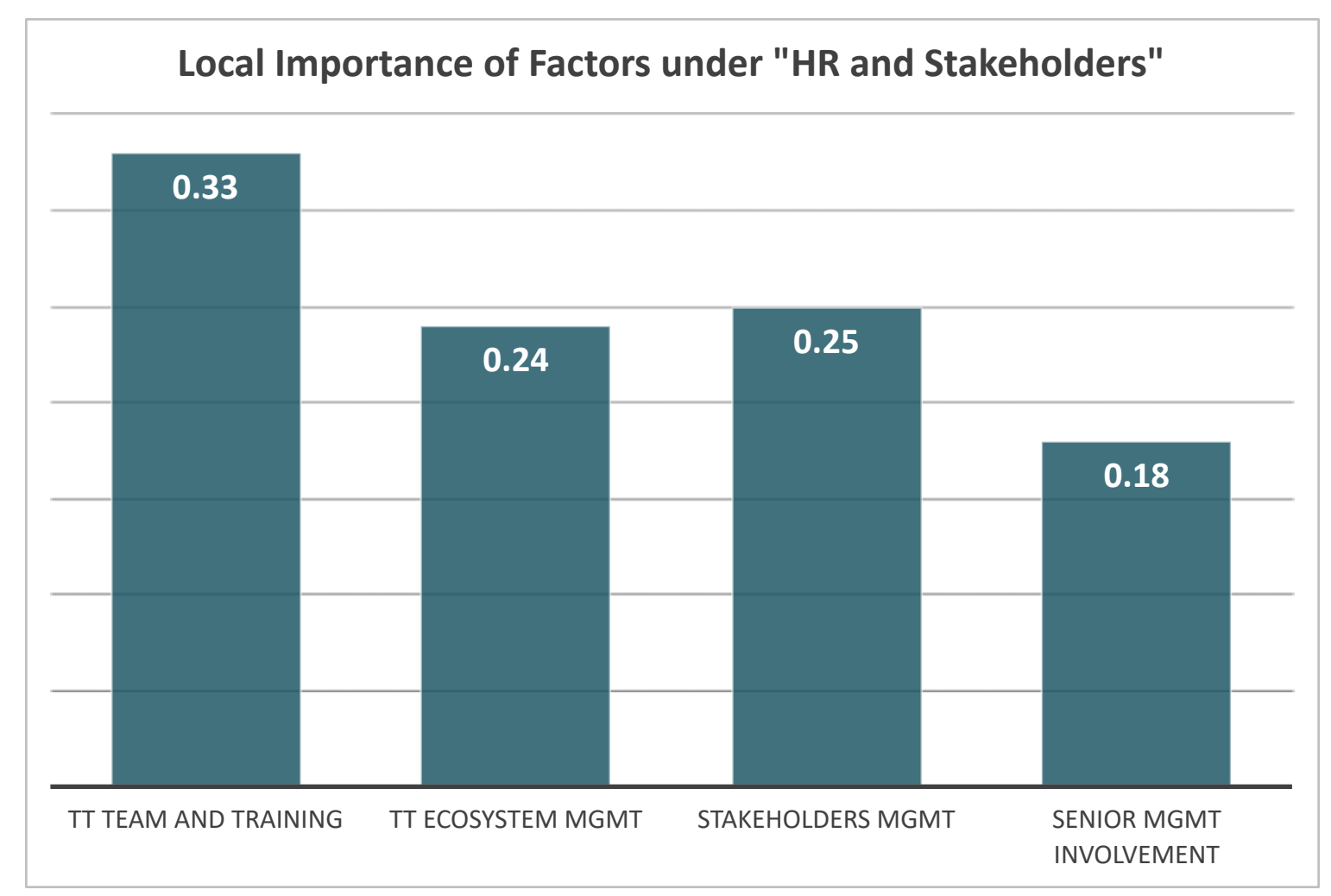

Figure 18 - HR and Stakeholders Factors Quantification Chart

The table and chart above show the local importance of factors under the "HR and Stakeholders" perspective, i.e., the level of contribution of each factor towards their specific perspective, not towards the model as a whole. The factor "TT and Training" is ranked as the most important (33\%). The second most important is "Stakeholders management", with 25\%, closely followed by "TT Ecosystem Management" (24\%). The least important factor within this perspective is "Senior Management Involvement", with 18\%. Disagreement and inconsistency levels are within the acceptable range. 
Table 52 - Organizational Culture Factors Quantification Results

\begin{tabular}{|c|c|c|c|c|c|c|c|}
\hline \multirow{3}{*}{ Panel } & Expert & $\begin{array}{c}\text { Communication } \\
\text { and knowledge } \\
\text { mgmt }\end{array}$ & Funding & $\begin{array}{c}\text { Absorptive } \\
\text { capacity }\end{array}$ & $\begin{array}{c}\text { Innovative } \\
\text { Culture }\end{array}$ & $\begin{array}{c}\text { Long-range } \\
\text { planning }\end{array}$ & Inconsistency \\
\hline \multirow{6}{*}{} & 14 & 0.23 & 0.15 & 0.15 & 0.24 & 0.22 & 0.05 \\
\cline { 2 - 9 } & 8 & 0.25 & 0.15 & 0.23 & 0.25 & 0.12 & 0.02 \\
\cline { 2 - 9 } & 11 & 0.24 & 0.29 & 0.20 & 0.15 & 0.11 & 0.04 \\
\cline { 2 - 9 } & 22 & 0.31 & 0.12 & 0.06 & 0.24 & 0.27 & 0.02 \\
\cline { 2 - 9 } & 1 & 0.26 & 0.25 & 0.11 & 0.26 & 0.12 & 0.09 \\
\cline { 2 - 8 } & 16 & 0.23 & 0.16 & 0.25 & 0.20 & 0.15 & 0.04 \\
\cline { 2 - 8 } & 32 & 0.23 & 0.29 & 0.14 & 0.21 & 0.12 & 0.02 \\
\cline { 2 - 8 } & 36 & 0.41 & 0.09 & 0.16 & 0.22 & 0.12 & 0.14 \\
\cline { 2 - 9 } & Mean & $\mathbf{0 . 2 7}$ & $\mathbf{0 . 1 9}$ & $\mathbf{0 . 1 6}$ & $\mathbf{0 . 2 2}$ & 0.15 & 0.055 \\
\hline
\end{tabular}

\section{Local Importance of Factors under "Organizational Culture"}

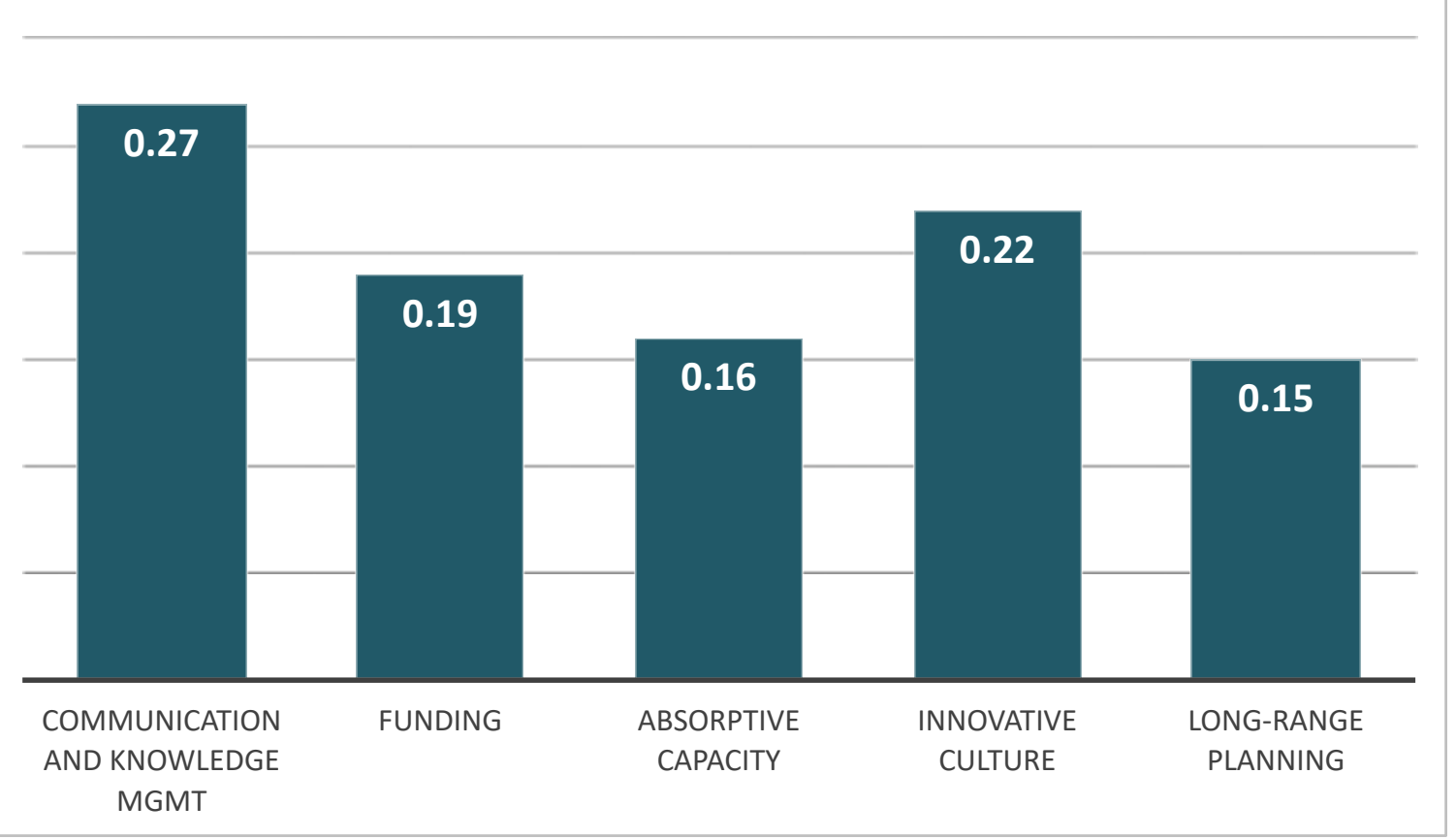

Figure 19 - Organizational Culture Factors Quantification Chart

The table and chart above show the local importance of factors under the "Organizational Culture" perspective, i.e., the level of contribution of each factor towards their specific perspective, not towards the model as a whole. The factor "Communication and Knowledge Management" is ranked as the most important 
(27\%). The second most important is "Innovative Culture", with $22 \%$. The third one is "Funding", with 19\%. The two least important factors under this perspective are "Absorptive Capacity" and "Long-range Planning", with 16\% and 15\% of importance levels, respectively. The disagreement level for Panel 9 is acceptable (less than 10\%), and all experts, except for Expert 36, have acceptable inconsistency levels. Since Expert 36 has an inconsistency level higher than the acceptable threshold of 10\%, his judgments would have to be redone or disregarded. Before going in that direction, however, a closer look at that expert's specific case was taken in light of theoretical innovations in the calculation of individual inconsistency levels. In 2016, Dr. Mustafa Abbas developed the root sum of variances (RSV), which was a new method and a new rationale for calculating and interpreting inconsistency levels for HDM models. [248]. During his research he found that the general rule of having $10 \%$ as a maximum could be too strict in some cases, especially when the expert is dealing with multiple criteria (as the number of criteria increase, pairwise comparisons increase exponentially). Based on that limitation, he set up to develop a new method to calculate the inconsistency and a scale of acceptable inconsistency levels, which varies depending on the number of variables involved (the more variables, the higher is the acceptable inconsistency) and the level of confidence.

Data from Expert 36 in Panel 9 was used to recalculate the inconsistency level using the RSV method, and the result was 0.30. According to Abbas' scale, for a level of confidence of $90 \%$ (alpha $=0.1$ ) and five variables, the threshold would be 0.33 . Since the calculated level is smaller than the threshold, judgments from Expert 36 in 
Panel 9 were kept. More information on the RSV calculation can be found in Appendix

A.

Table 53 - Technical Factors Quantification Results

\begin{tabular}{|c|c|c|c|c|c|}
\hline Panel & Expert & $\begin{array}{c}\text { TRL } \\
\text { assessment }\end{array}$ & $\begin{array}{l}\text { Technology } \\
\text { valuation }\end{array}$ & $\begin{array}{c}\text { Proposal } \\
\text { assessment }\end{array}$ & Inconsistency \\
\hline \multirow{7}{*}{10} & 26 & 0.18 & 0.41 & 0.41 & 0.00 \\
\hline & 17 & 0.35 & 0.33 & 0.32 & 0.04 \\
\hline & 3 & 0.12 & 0.54 & 0.34 & 0.04 \\
\hline & 5 & 0.21 & 0.48 & 0.31 & 0.00 \\
\hline & 37 & 0.19 & 0.55 & 0.26 & 0.03 \\
\hline & Mean & 0.21 & 0.46 & \begin{tabular}{|l|}
0.33 \\
\end{tabular} & \\
\hline & \multicolumn{4}{|c|}{ Disagreement } & 0.064 \\
\hline
\end{tabular}

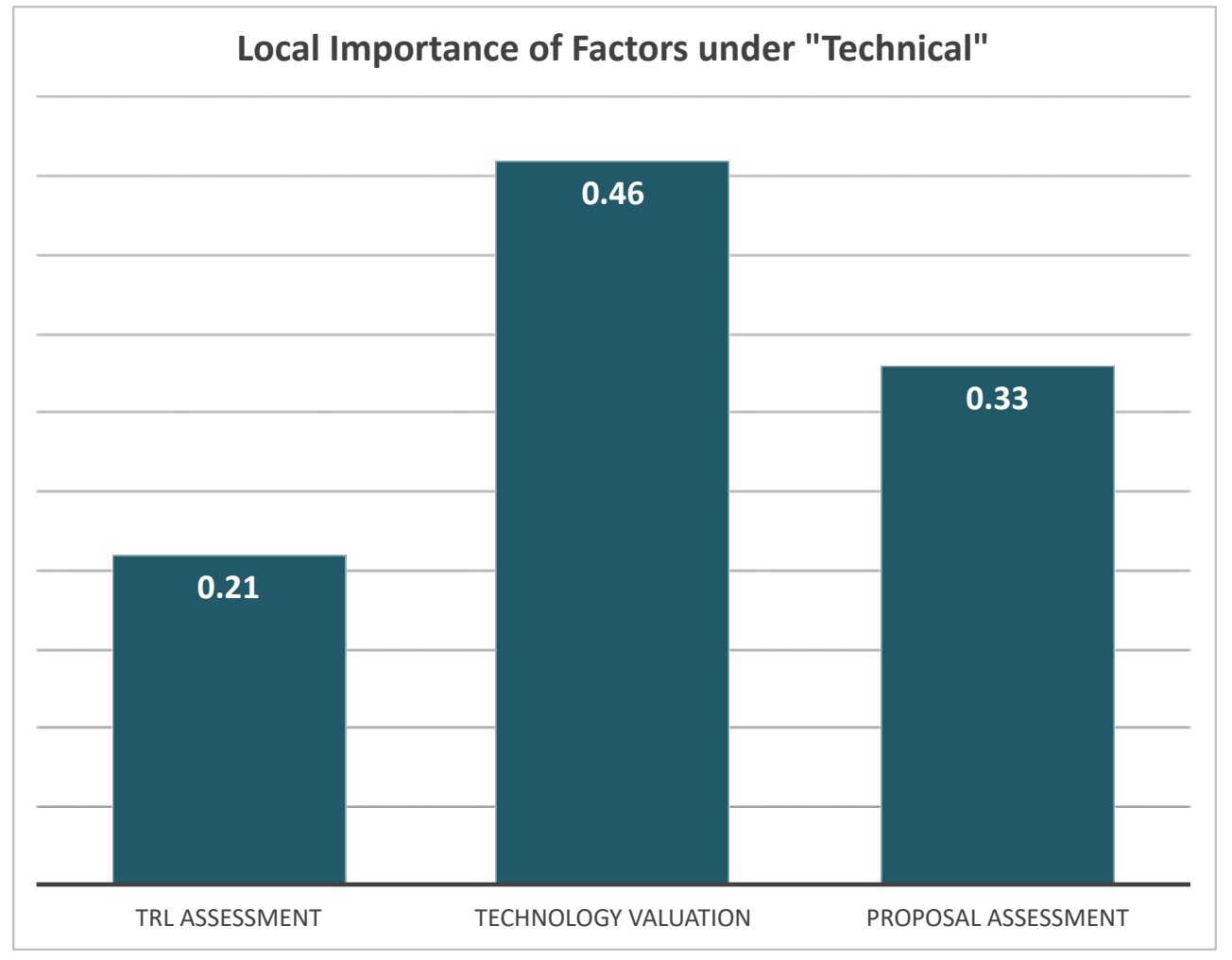

Figure 20 - Technical Factors Quantification Chart

The table and chart above show the local importance of factors under the "Technical" perspective, i.e., the level of contribution of each factor towards their 
specific perspective, not towards the model as a whole. The factor "Technology valuation" is ranked as the most important (46\%). The second most important is "Proposal Assessment", with 33\%, and the third one is "TRL Assessment" with 21\%. Disagreement and inconsistency levels are within the acceptable range.

Table 54 - Process Factors Quantification Results

\begin{tabular}{|c|c|c|c|c|c|c|}
\hline Panel & Expert & $\begin{array}{c}\text { Benefit } \\
\text { mgmt }\end{array}$ & Risk mgmt & $\begin{array}{c}\text { Outcomes } \\
\text { and } \\
\text { decisions }\end{array}$ & $\begin{array}{c}\mathrm{TT} \\
\text { mechanisms } \\
\text { mgmt }\end{array}$ & Inconsistency \\
\hline \multirow{9}{*}{11} & 13 & 0.29 & 0.29 & 0.13 & 0.29 & 0 \\
\hline & 26 & 0.37 & 0.29 & 0.20 & 0.14 & 0 \\
\hline & 21 & 0.25 & 0.25 & 0.25 & 0.25 & 0 \\
\hline & 14 & 0.22 & 0.33 & 0.22 & 0.22 & 0 \\
\hline & 1 & 0.26 & 0.26 & 0.34 & 0.14 & 0.05 \\
\hline & 7 & 0.25 & 0.25 & 0.25 & 0.25 & 0 \\
\hline & 36 & 0.18 & 0.05 & 0.67 & 0.10 & 0.06 \\
\hline & Mean & 0.26 & 0.25 & 0.29 & 0.20 & \\
\hline & \multicolumn{5}{|r|}{ Disagreement } & 0.081 \\
\hline
\end{tabular}




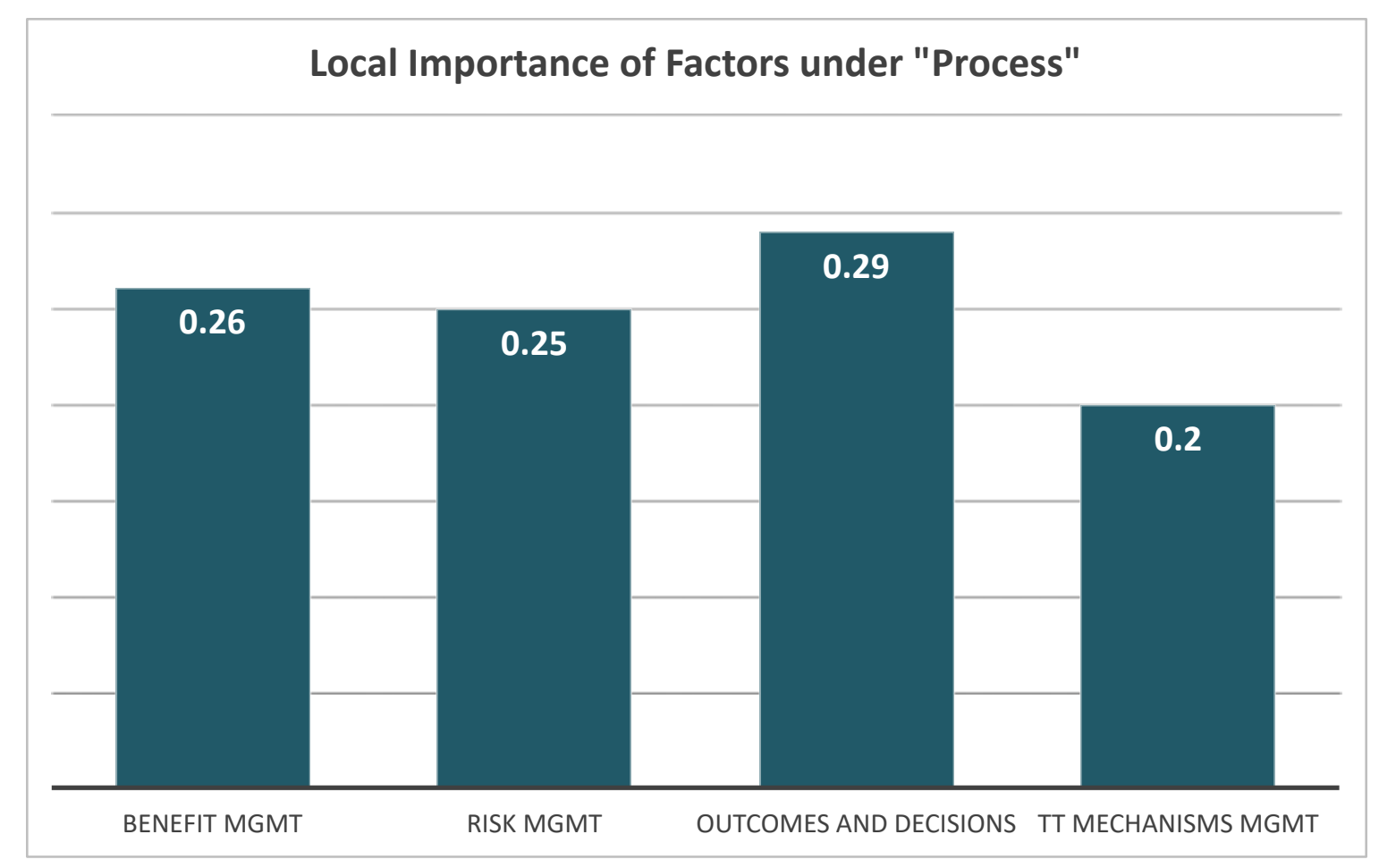

Figure 21 - Process Factors Quantification Chart

The table and chart above show the local importance of factors under the "Process" perspective, i.e., the level of contribution of each factor towards their specific perspective, not towards the model as a whole. The factor "Outcomes and Decisions" is ranked as the most important (29\%). The second most important is "Benefit Management", with 26\%, closely followed by "Risk Management" (25\%). The least important factor within this perspective is "TT Mechanisms Management", with 20\%. Disagreement and inconsistency levels are within the acceptable range. 
Table 55 - Strategic Alignment Factors Quantification Results

\begin{tabular}{|c|c|c|c|c|c|c|c|}
\hline Panel & Expert & $\begin{array}{c}\text { Value, } \\
\text { impact and } \\
\text { applicability } \\
\text { mgmt } \\
\end{array}$ & $\begin{array}{l}\text { Business } \\
\text { plan and } \\
\text { use case }\end{array}$ & $\begin{array}{c}\text { TT planning, } \\
\text { control and } \\
\text { flexibility }\end{array}$ & $\begin{array}{l}\text { Continuity } \\
\text { of TT } \\
\text { process }\end{array}$ & $\begin{array}{c}\text { Parallel } \\
\text { processes } \\
\text { integration }\end{array}$ & Inconsistency \\
\hline \multirow{10}{*}{12} & 31 & 0.31 & 0.26 & 0.20 & 0.12 & 0.11 & 0.01 \\
\hline & 8 & 0.22 & 0.13 & 0.21 & 0.19 & 0.25 & 0.07 \\
\hline & 24 & 0.40 & 0.13 & 0.13 & 0.14 & 0.2 & 0 \\
\hline & 28 & 0.39 & 0.16 & 0.23 & 0.11 & 0.11 & 0.02 \\
\hline & 22 & 0.26 & 0.24 & 0.18 & 0.15 & 0.17 & 0.01 \\
\hline & 6 & 0.17 & 0.14 & 0.19 & 0.27 & 0.23 & 0.01 \\
\hline & 16 & 0.19 & 0.16 & 0.30 & 0.22 & 0.13 & 0.03 \\
\hline & 39 & 0.31 & 0.24 & 0.22 & 0.13 & 0.11 & 0.02 \\
\hline & Mean & 0.28 & 0.18 & 0.21 & 0.17 & 0.16 & \\
\hline & \multicolumn{6}{|c|}{ Disagreement } & 0.056 \\
\hline
\end{tabular}

\section{Local Importance of Factors under "Strategic Alignment"}

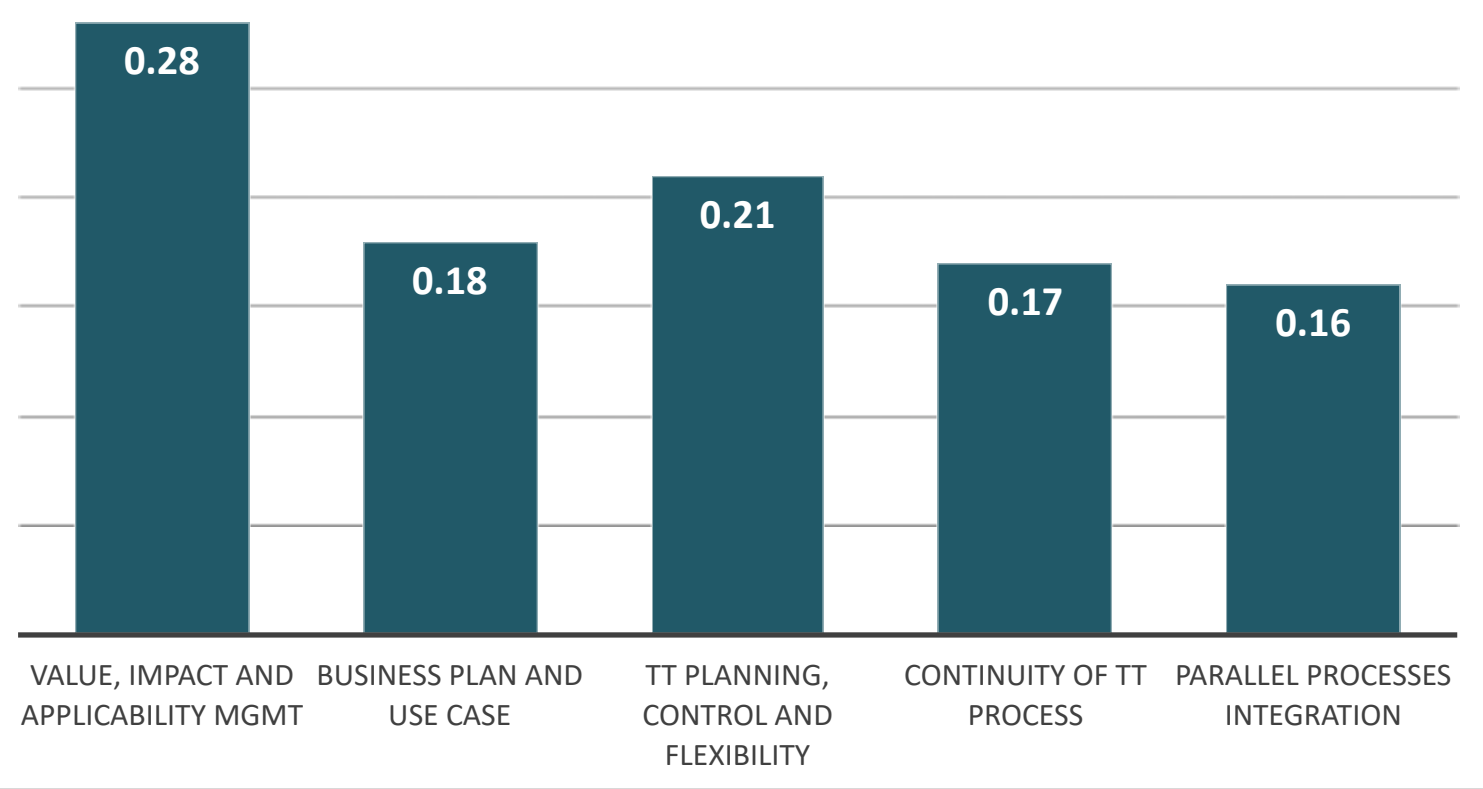

Figure 22 - Strategic Alignment Factors Quantification Chart

The table and chart above show the local importance of factors under the "Strategic Alignment" perspective, i.e., the level of contribution of each factor towards their specific perspective, not towards the model as a whole. The factor "Value, Impact and Applicability Management" is ranked as the most important (28\%). The second 
most important is "TT Planning, Control and Flexibility", with 21\%. The last three factors are similarly ranked, with $18 \%, 17 \%$ and $16 \%$ for "Business Plan and Use Case”, "Continuity of TT Process", and "Parallel Processes Integration”. Disagreement and inconsistency levels are within the acceptable range.

\section{Summary of Model Quantification}

Table 56 and Figure 23 summarize the weights obtained for perspectives and factors through the experts' judgments. The table includes local and global importance values for each factor. 
Table 56 - Model Quantification Results Summary Table

\begin{tabular}{|c|c|c|c|c|}
\hline \multicolumn{2}{|c|}{ Perspectives } & \multicolumn{3}{|c|}{ Factors } \\
\hline Name & Value & Name & $\begin{array}{l}\text { Local } \\
\text { value }\end{array}$ & $\begin{array}{l}\text { Global } \\
\text { value }\end{array}$ \\
\hline \multirow{4}{*}{$\begin{array}{c}\text { HR and } \\
\text { Stakeholders }\end{array}$} & \multirow{4}{*}{0.23} & TT team and training & 0.33 & 0.07 \\
\hline & & TT ecosystem mgmt & 0.24 & 0.05 \\
\hline & & Stakeholders mgmt & 0.25 & 0.06 \\
\hline & & Senior mgmt involvement & 0.18 & 0.04 \\
\hline \multirow{5}{*}{$\begin{array}{c}\text { Organizational } \\
\text { Culture }\end{array}$} & \multirow{5}{*}{0.18} & Communication and knowledge mgmt & 0.27 & 0.05 \\
\hline & & Funding & 0.19 & 0.03 \\
\hline & & Absorptive capacity & 0.16 & 0.03 \\
\hline & & Long-range planning & 0.15 & 0.03 \\
\hline & & Innovative Culture & 0.22 & 0.04 \\
\hline \multirow{3}{*}{ Technical } & \multirow{3}{*}{0.17} & TRL assessment & 0.21 & 0.04 \\
\hline & & Technology valuation & 0.46 & 0.08 \\
\hline & & Proposal assessment & 0.33 & 0.06 \\
\hline \multirow{4}{*}{ Process } & \multirow{4}{*}{0.17} & Benefit mgmt & 0.26 & 0.04 \\
\hline & & Risk mgmt & 0.25 & 0.04 \\
\hline & & Outcomes and decisions & 0.29 & 0.05 \\
\hline & & TT mechanisms mgmt & 0.20 & 0.03 \\
\hline \multirow{5}{*}{$\begin{array}{c}\text { Strategic } \\
\text { Alignment }\end{array}$} & \multirow{5}{*}{0.24} & Value, impact and applicability mgmt & 0.28 & 0.07 \\
\hline & & Business plan and use case & 0.18 & 0.04 \\
\hline & & TT planning, control and flexibility & 0.21 & 0.05 \\
\hline & & Continuity of TT process & 0.17 & 0.04 \\
\hline & & Parallel processes integration & 0.16 & 0.04 \\
\hline
\end{tabular}




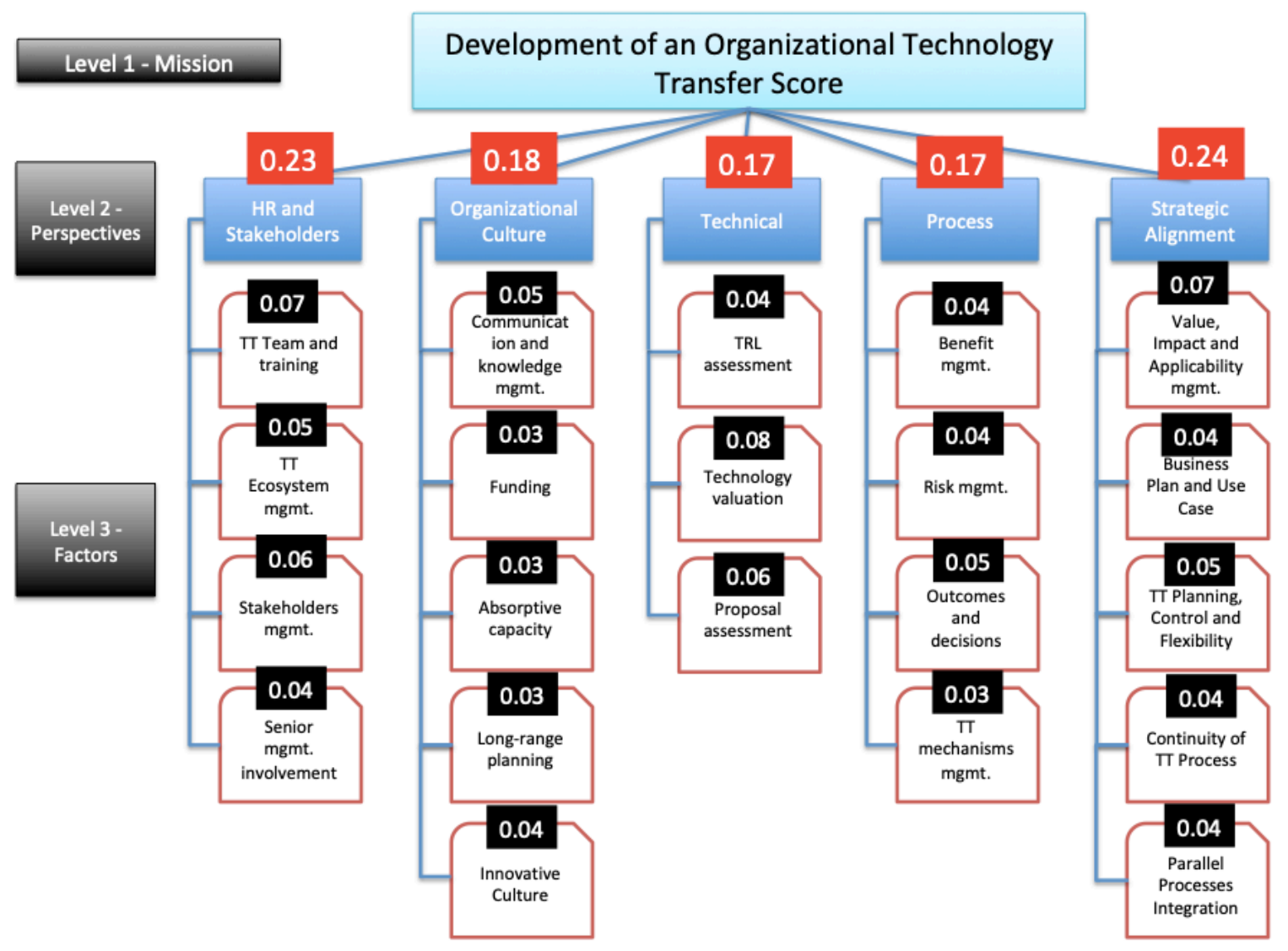

Figure 23 - Model Quantification Results Summary Figure

Overall, the most important factors are: “Technology Valuation”, contributing 8\% to the final TT score; "TT Team and Training", "Value, Impact and Applicability", contributing 7\% each to the final score; "Stakeholders Management", "Proposal Assessment", contributing 6\% each to the final score. Conversely, the least important factors, contributing 3\% each to the final score are: "Funding", "Absorptive Capacity", “Long-range Planning”, “TT Mechanisms Management”. 


\section{Desirability Curves}

The following present the desirability levels and curves intended to measure each of the twenty-one factors in the model. The desirability levels were presented to and discussed with an executive who has solid experience in technology transfer in both academia and in the energy industry. The quantification of the levels and consequent desirability curves resulted from the discussion.

\section{Human Resources and Stakeholders Perspective}

\section{TT Team and Training}

- No dedicated TT team and no training.

- Dedicated TT team; not multidisciplinary; no training.

- Dedicated TT team; slightly multidisciplinary (with representatives from more than one department); either no training or informal training.

- Dedicated TT team; multidisciplinary (with representatives from all relevant departments); formal training.

- Dedicated TT team; multidisciplinary (with representatives from all relevant departments); formal periodic training (quarterly-based or more often). 


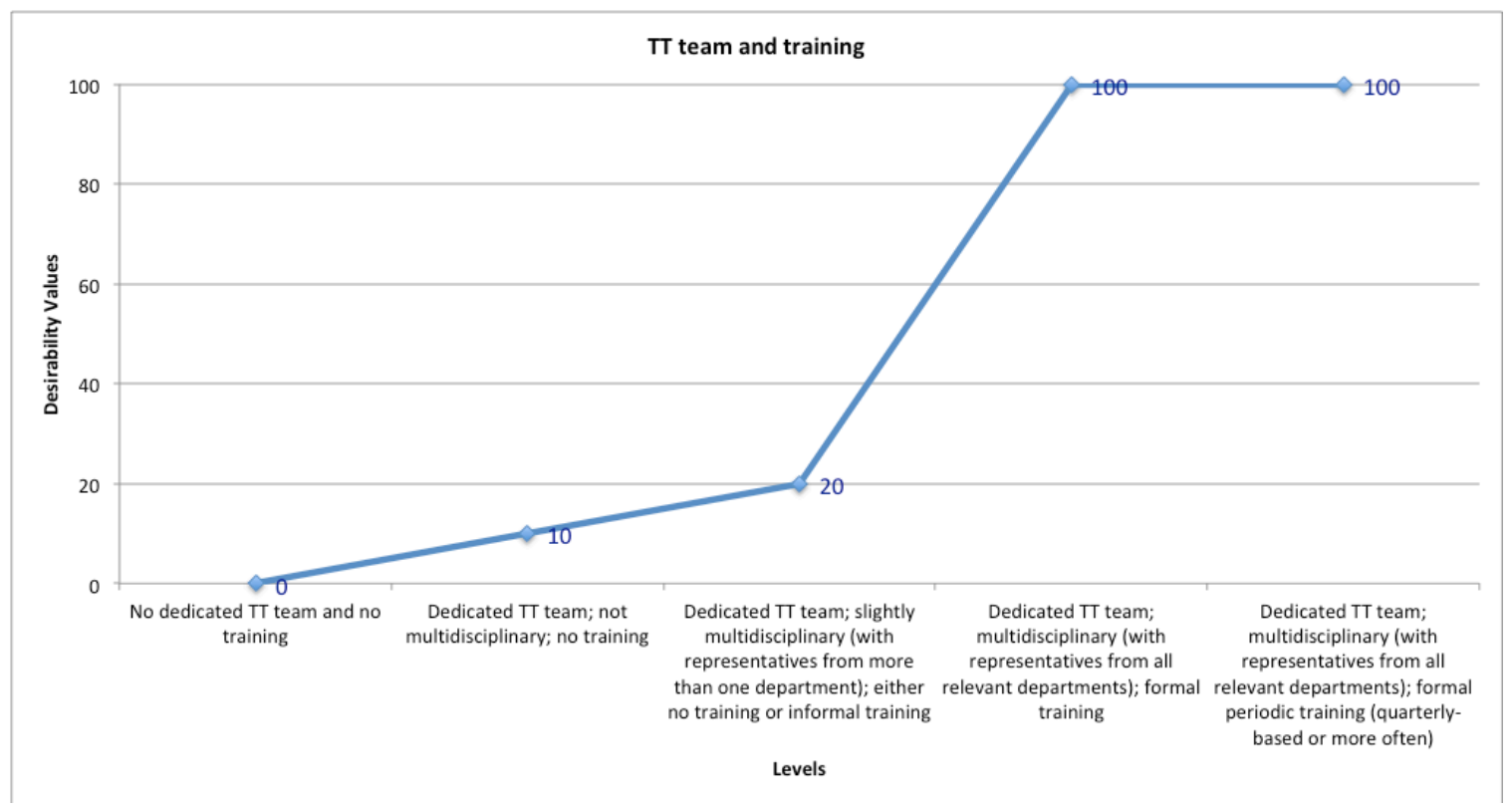

Figure 24 - TT team and Training Curve

\section{TT Ecosystem Management}

- No interaction with TT ecosystem entities whatsoever.

- Sparse interaction with TT ecosystem (less than monthly) with reluctance to share information or engage in cooperation.

- Frequent interaction (at least monthly) with unstructured, ad-hoc discussions.

- Frequent interaction (at least monthly) with structured discussions in the form of summits / events with up to three TT ecosystem entities.

- Frequent interaction (at least monthly), and formal agreements with more than three TT ecosystem entities. 


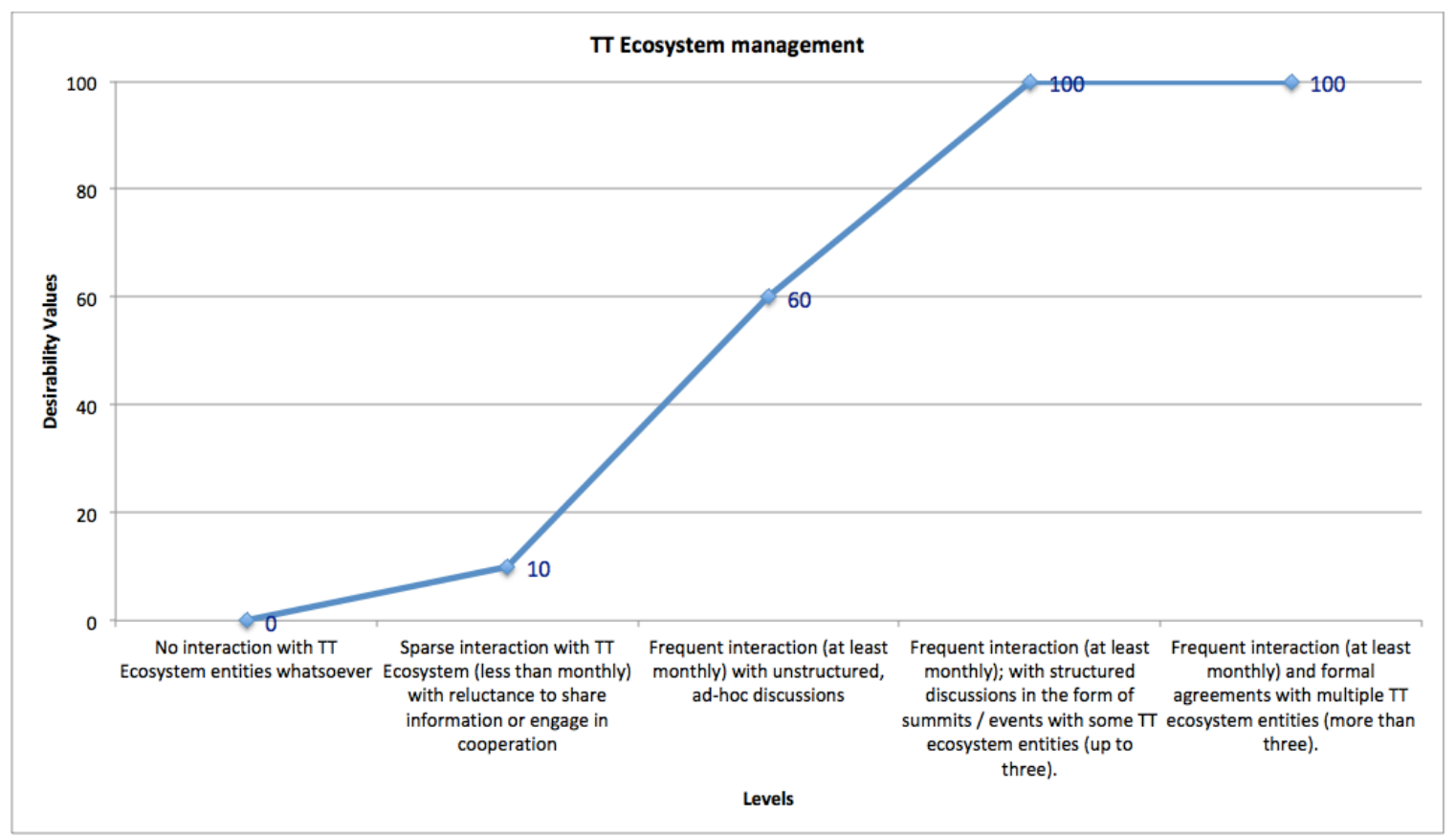

Figure 25 - TT Ecosystem Management Curve

\section{Stakeholders Management}

- $\quad$ TT process is limited to the TT team alone. No one else participates.

- TT team has sparse contact (less than monthly) with internal stakeholders (and external, if any); information goes one way (from the stakeholders, but with solicitation).

- TT team has frequent contact (at least monthly) with internal stakeholders (and external, if any); information goes one way (from the stakeholders, but with solicitation).

- TT team has frequent contact (at least monthly) with internal stakeholders (and external, if any); information goes one way (from the stakeholders and without solicitation). 
- TT team has frequent contact (at least monthly) with internal stakeholders (and external, if any); information goes both ways (from and to stakeholders).

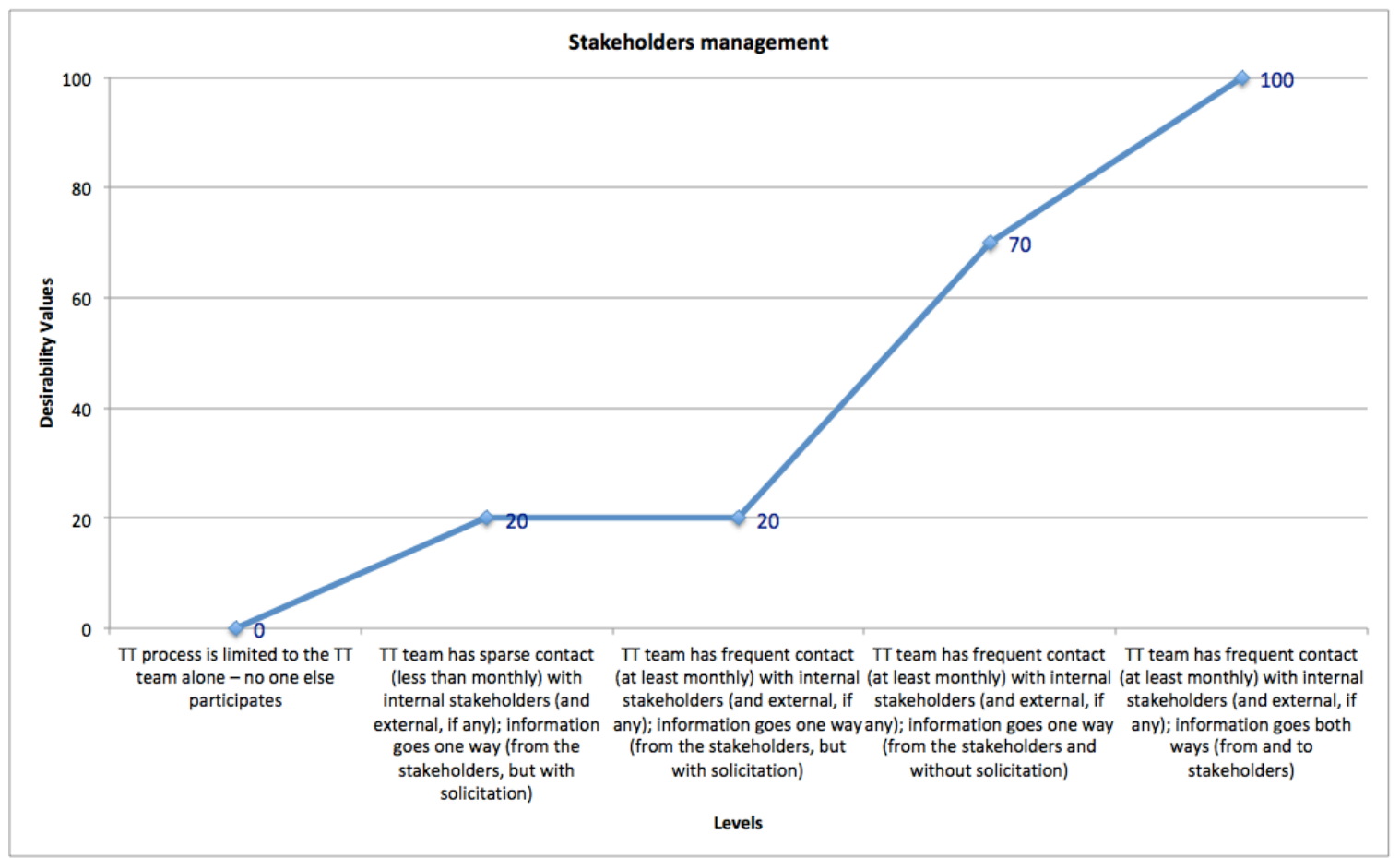

Figure 26 - Stakeholders Management Curve

\section{Senior Management Involvement}

- There is no senior management interest in the process.

- TT process is supported by middle management, but their engagement is not consistent.

- Middle management is actively engaged in the TT process, but senior management still does not openly support it.

- Middle management is actively engaged in the TT process. Senior management is aware of the TT process, but their engagement is not consistent. 
- There is evidence of consistent engagement and support for the TT process at all levels of the organization, including the existence of TT champions endorsed by senior management.

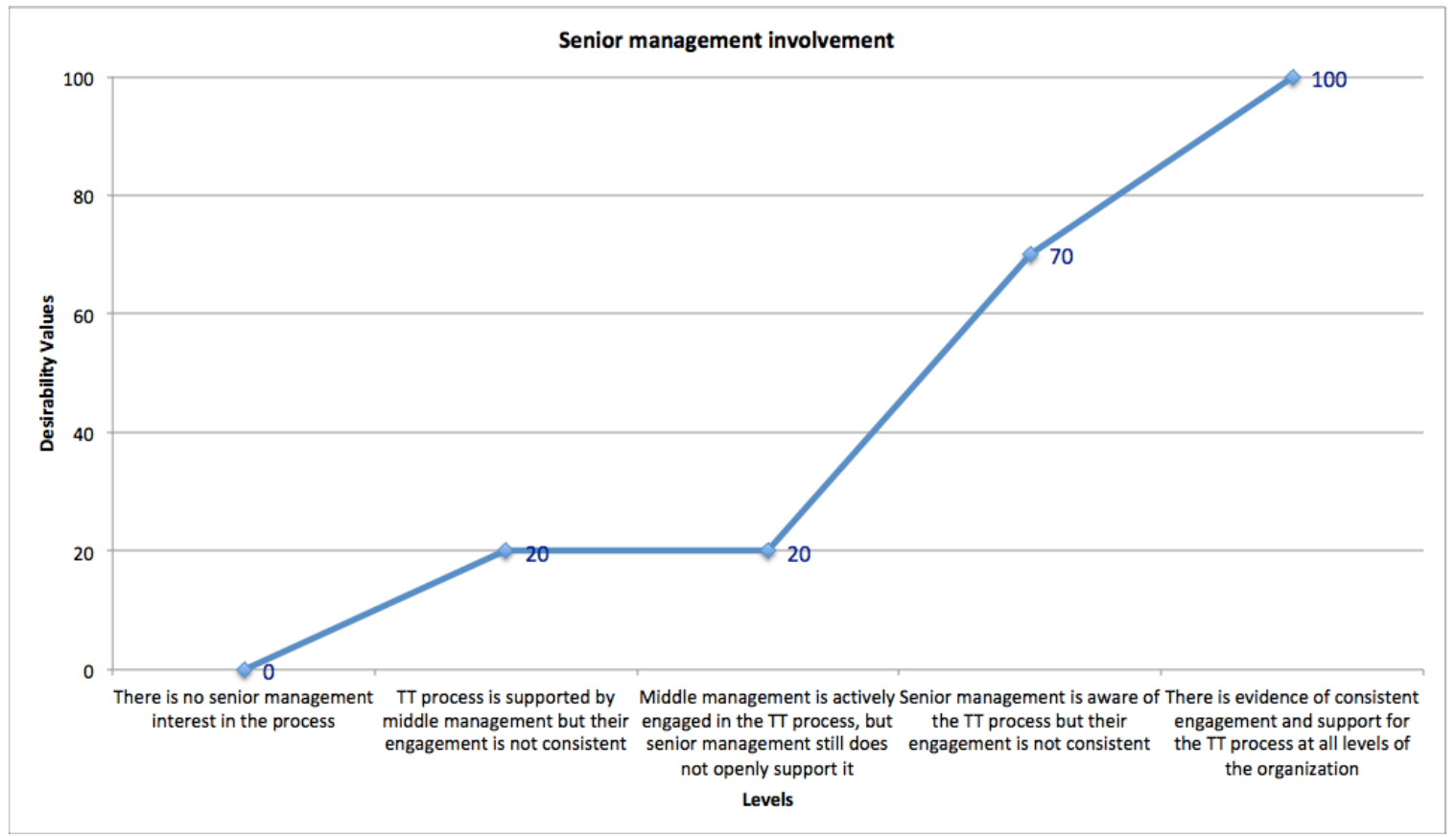

Figure 27 - Senior Management Involvement Curve

\section{Organizational Culture Perspective}

\section{Communication and Knowledge Management}

- Inter-departmental communication is weak; relevant information is not transferred from one department to another without solicitation.

- Inter-departmental communication is weak; only vital information is transferred from one department to another without solicitation.

- Inter-departmental communication is strong; relevant information is transferred from one department to another without solicitation. 
- Inter-departmental communication is abundant and intense; knowledge transfer is an inherent behavior in the organization.

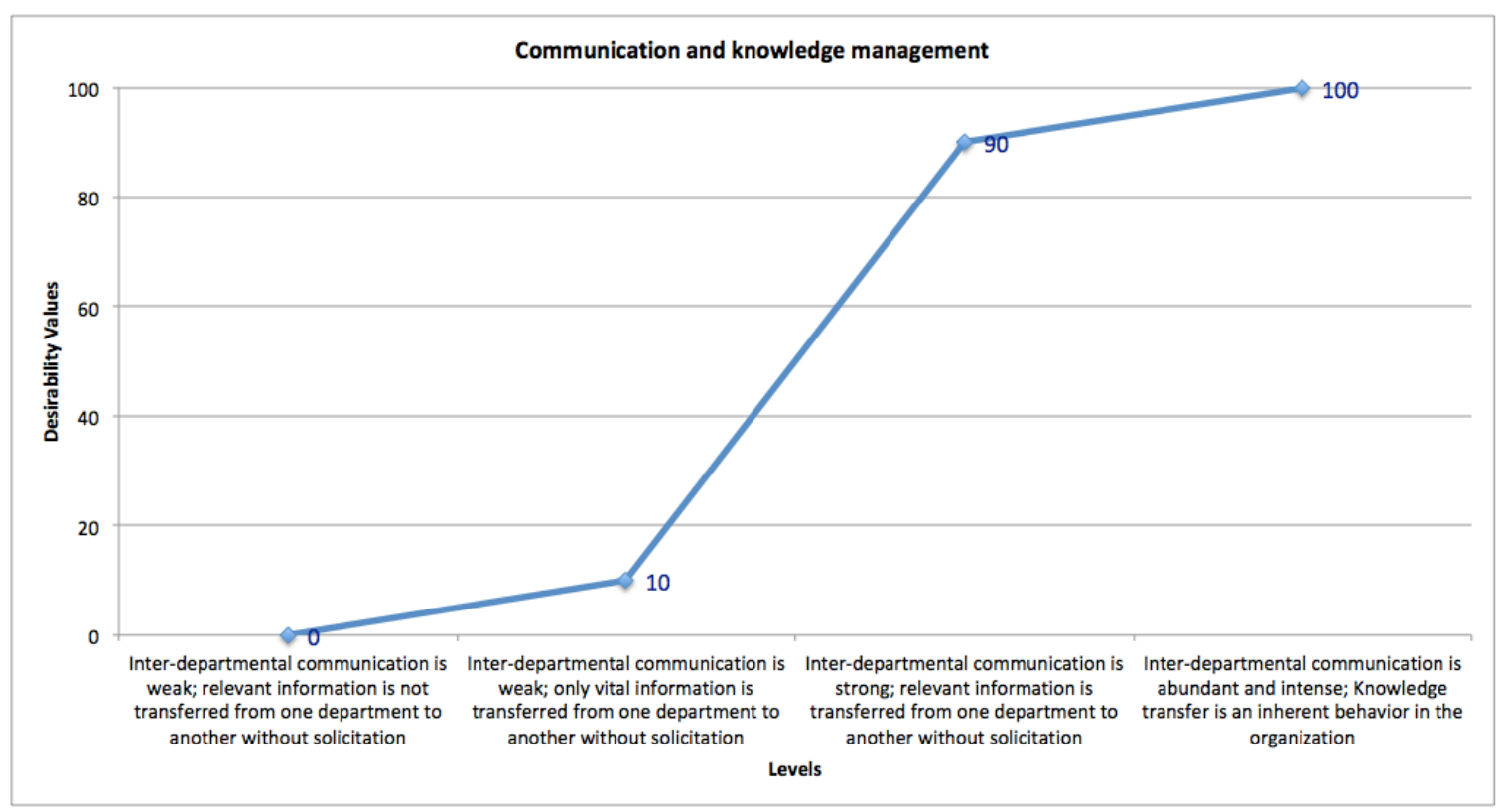

Figure 28 - Communication and Knowledge Management Curve

\section{Funding}

- No budget is allocated.

- $1 \%$ of R\&D budget is allocated to TT activities.

- Between $1 \%$ and $5 \%$ of R\&D budget is allocated to TT activities.

- Between $5 \%$ and $10 \%$ of R\&D budget is allocated to TT activities.

- More than $10 \%$ of R\&D budget is allocated to TT activities. 


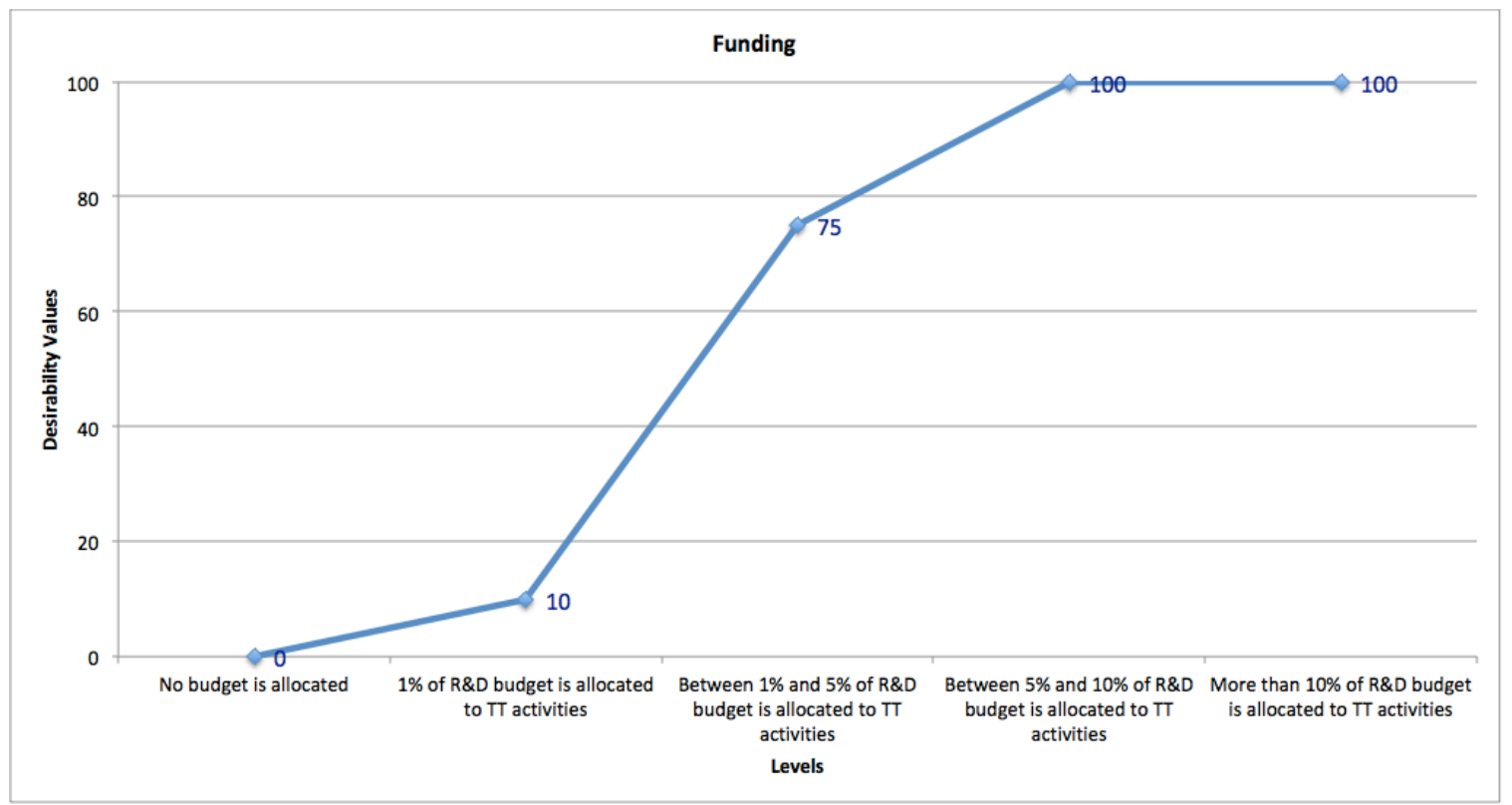

Figure 29 - Funding

\section{Absorptive Capacity}

- The organization does not have steady and successful partnerships, collaborative developments, or joint R\&D efforts with external parties.

- The organization steady and successful partnerships, collaborative development, or joint R\&D efforts and those amount up to $10 \%$ of its research portfolio.

- The organization steady and successful partnerships, collaborative development, or joint R\&D efforts and those amount to $10 \%$ to $25 \%$ of its research portfolio.

- The organization steady and successful partnerships, collaborative development, or joint $R \& D$ efforts and those amount to $25 \%$ to $50 \%$ of its research portfolio. 
- The organization steady and successful partnerships, collaborative development, or joint R\&D efforts and those amount to more than $50 \%$ of its research portfolio.

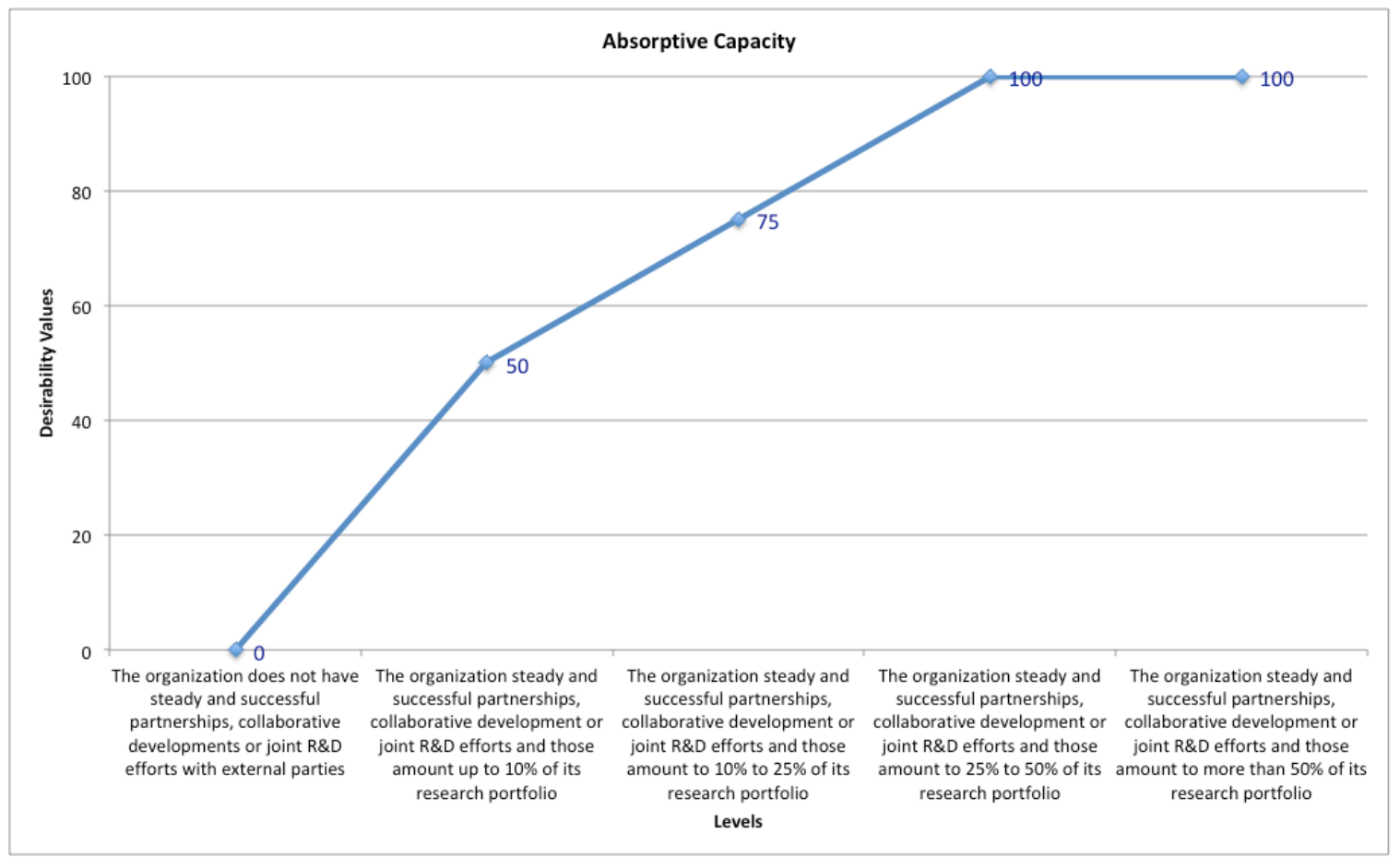

Figure 30 - Absorptive Capacity Curve

\section{Long-range Planning}

- Corporate and business strategies are not propagated.

- Informal corporate and business strategies are tacitly propagated.

- Formal corporate and business strategies exist, but are tacitly propagated.

- Formal corporate and business strategies exist, and are formally propagated.

- Formal corporate and business strategies exist, are formally propagated, and employees contrast the strategies against their daily activities. 


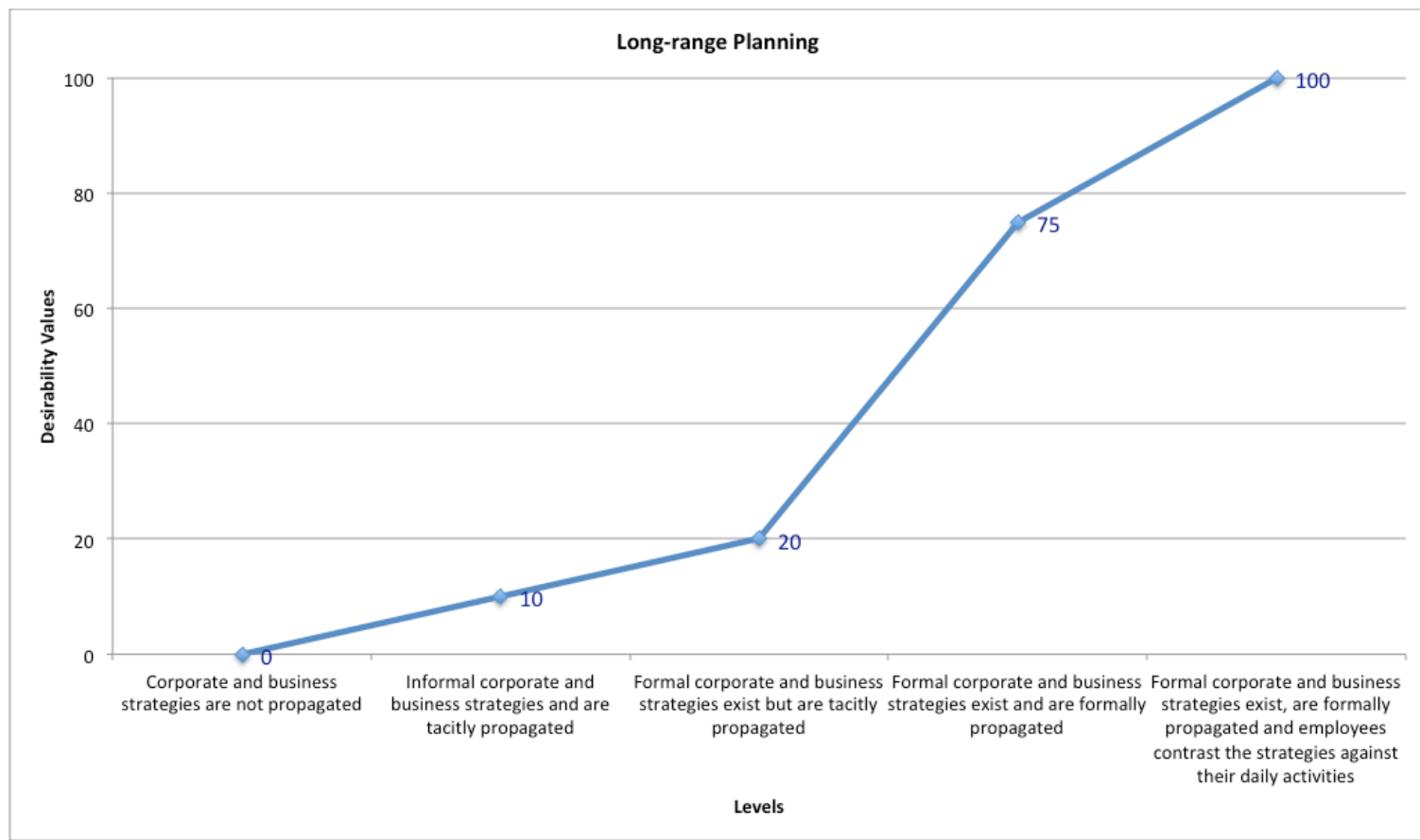

Figure 31 - Long Range Planning Curve

\section{Innovative Culture}

- Innovations / changes in business practices are discouraged, and face high resistance within the organization

- Innovations / changes in business practices have been tried, but have not been successfully implemented in the last three years

- Minor innovations / changes in business practices have been successfully implemented in the last three years

- Major innovations / changes in business practices have been successfully implemented in the last three years

- Major innovations / changes in business practices are constantly, and successfully implemented in the organization. 


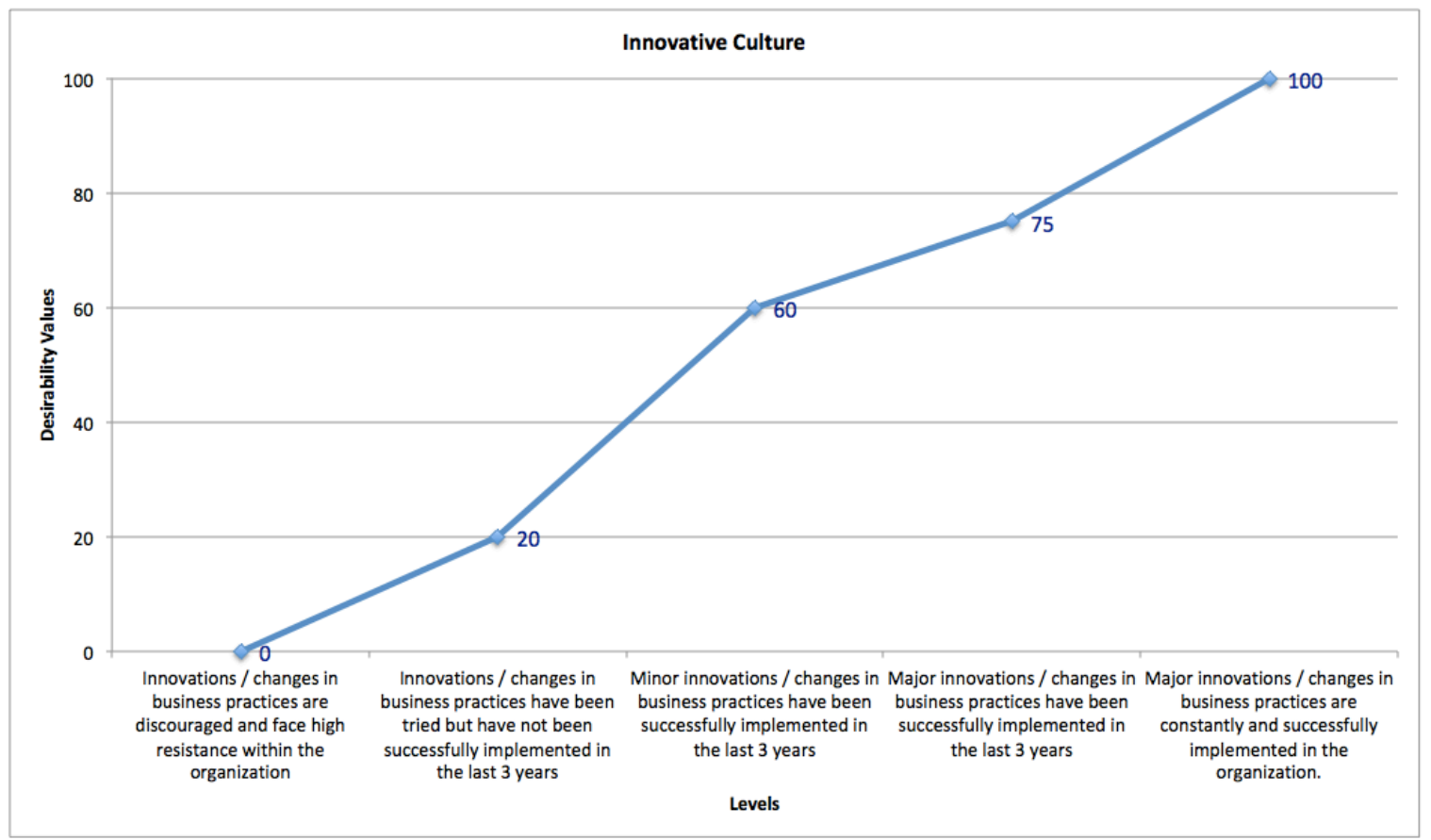

Figure 32 - Innovative Culture Curve

\section{Technical Perspective}

\section{TRL Assessment}

- No readiness assessment is conducted for the technologies being developed.

- Technology readiness is assessed, but does not inform the decision-making process in any ways.

- Technology readiness is assessed, and it informs the decision-making process regarding TT mechanisms and strategies.

- Readiness assessments are conducted concerning technology (TRL) and system readiness (IRL, SRL), and the assessments inform the decisionmaking process regarding TT mechanisms and strategies. 
- Readiness assessments are conducted concerning technology (TRL), system readiness (IRL, SRL), and R\&D degree of difficulty (RD3); the assessments inform the decision-making process regarding TT mechanisms and strategies.

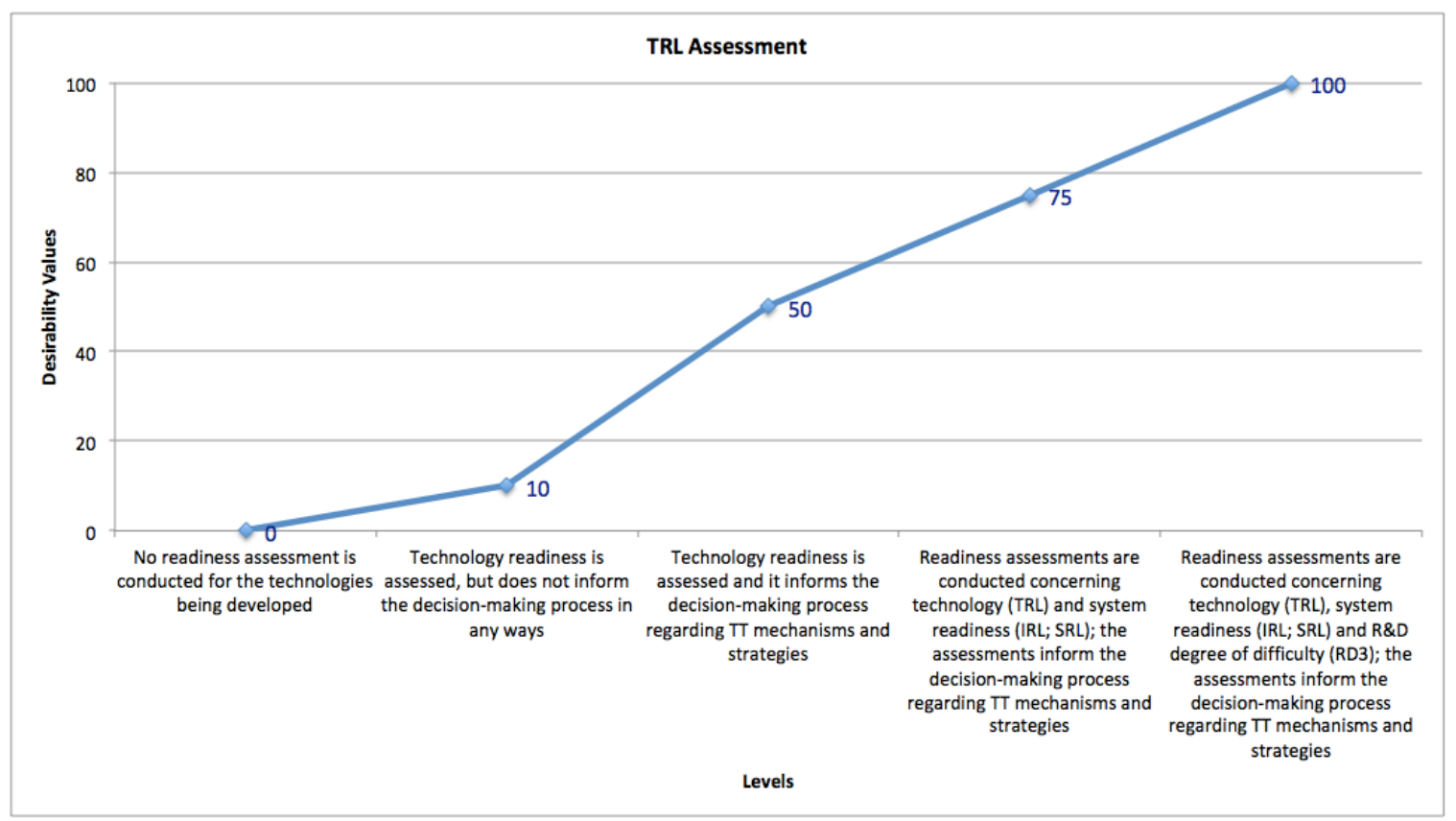

Figure 33 - TRL Assessment Curve

\section{Technology Valuation}

- No technology assessment studies are conducted throughout the technology development cycle.

- One technology assessment study is conducted at the beginning of the technology development cycle.

- Technology assessment studies are conducted at the beginning and at the mid-point of the technology development cycle. 
- Technology assessment studies are conducted at the beginning, at the midpoint, and at the end of the technology development cycle.

- Technology assessment studies are dynamically conducted throughout the technology development cycle, based on project and technology changes.

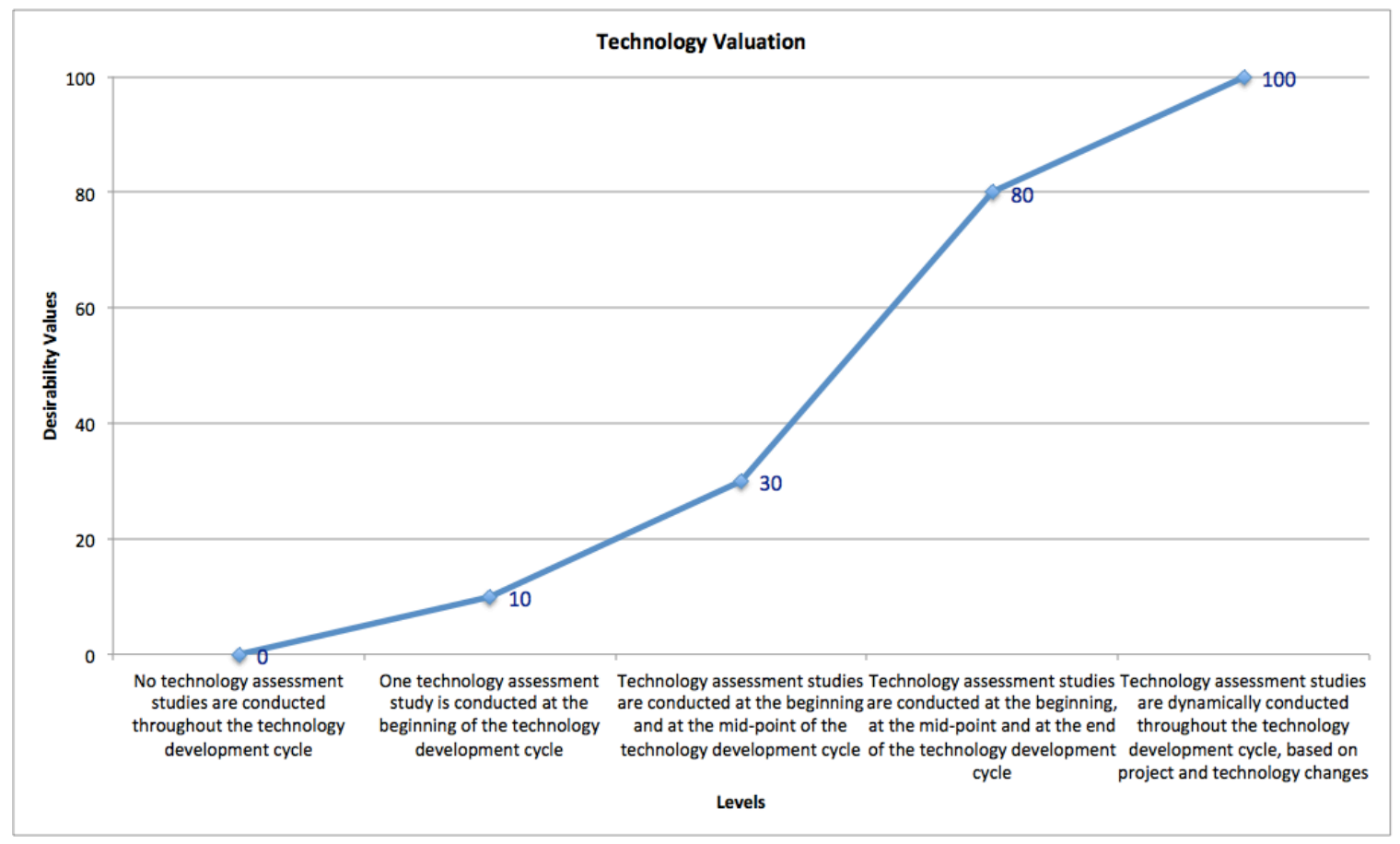

Figure 34 - Technology Valuation Curve

\section{Proposal Assessment}

- Research proposals are not methodically assessed.

- Research proposals are methodically and qualitatively assessed.

- Research proposals are methodically and quantitatively assessed.

- Research proposals are methodically and quantitatively assessed through more than one method. 
- Research proposals are methodically and quantitatively assessed through more than two methods.

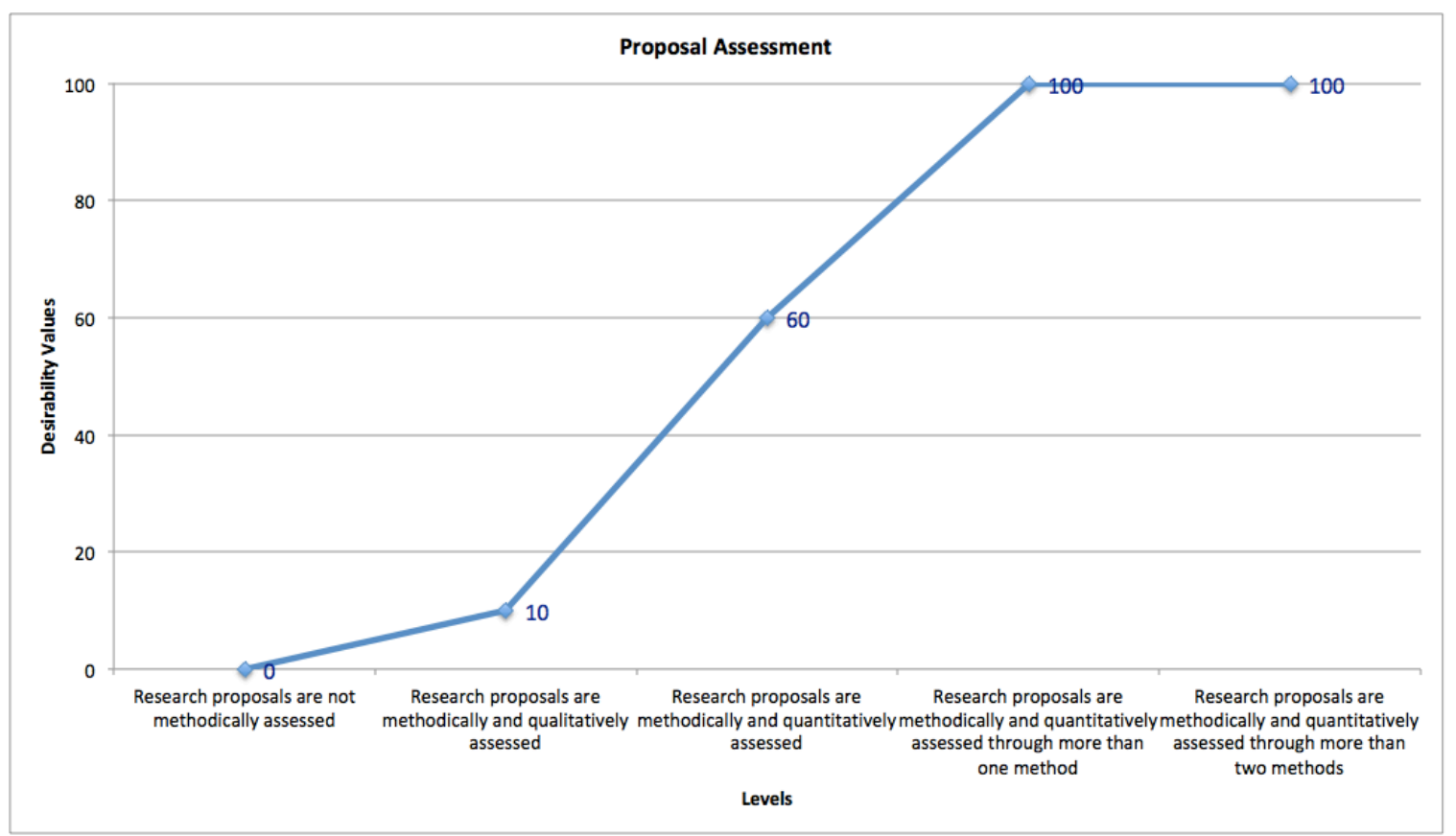

Figure 35 - Proposal Assessment Curve

\section{Process Perspective}

\section{Benefit Management}

- Project deliverables are seen as benefits, without key stakeholders' input or program considerations.

- Benefits are differentiated from project deliverables, but without key stakeholders' input or program considerations.

- Benefits are differentiated from project deliverables, classified and quantified, without stakeholders' input or program considerations.

- Benefits are classified, quantified and monitored. Benefits are confirmed by key stakeholders, and are mapped in terms of program benefits. 


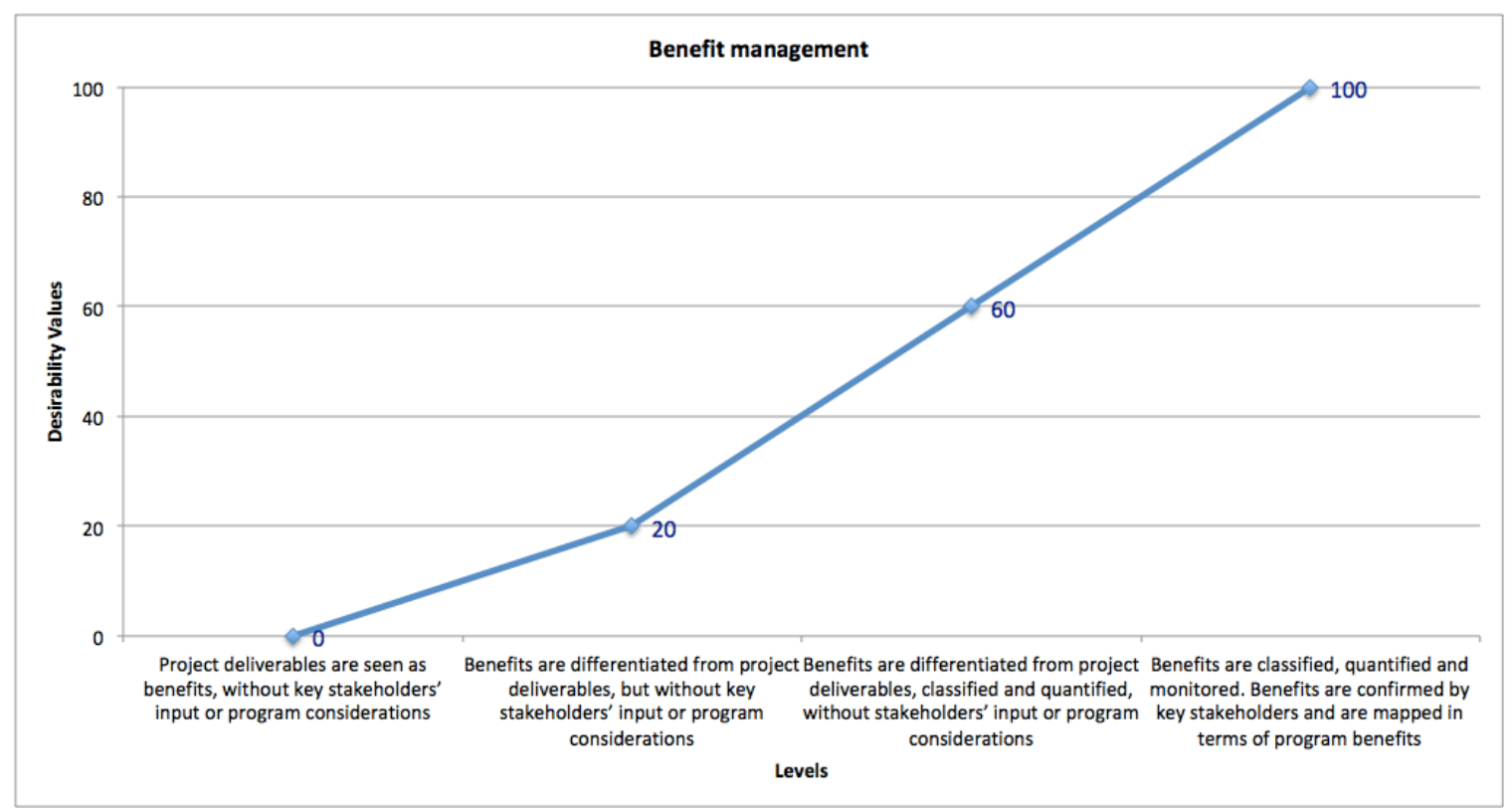

Figure 36 - Benefit Management Curve

\section{Risk Management}

- The project management perspective of risk is seen as enough.

- The TT risks and opportunities are identified.

- The TT risks and opportunities are identified and quantified.

- TT risks and opportunities are actively managed. Back-up plans are devised (risks); follow-on research plan and engagement with external parties are made (opportunities). 


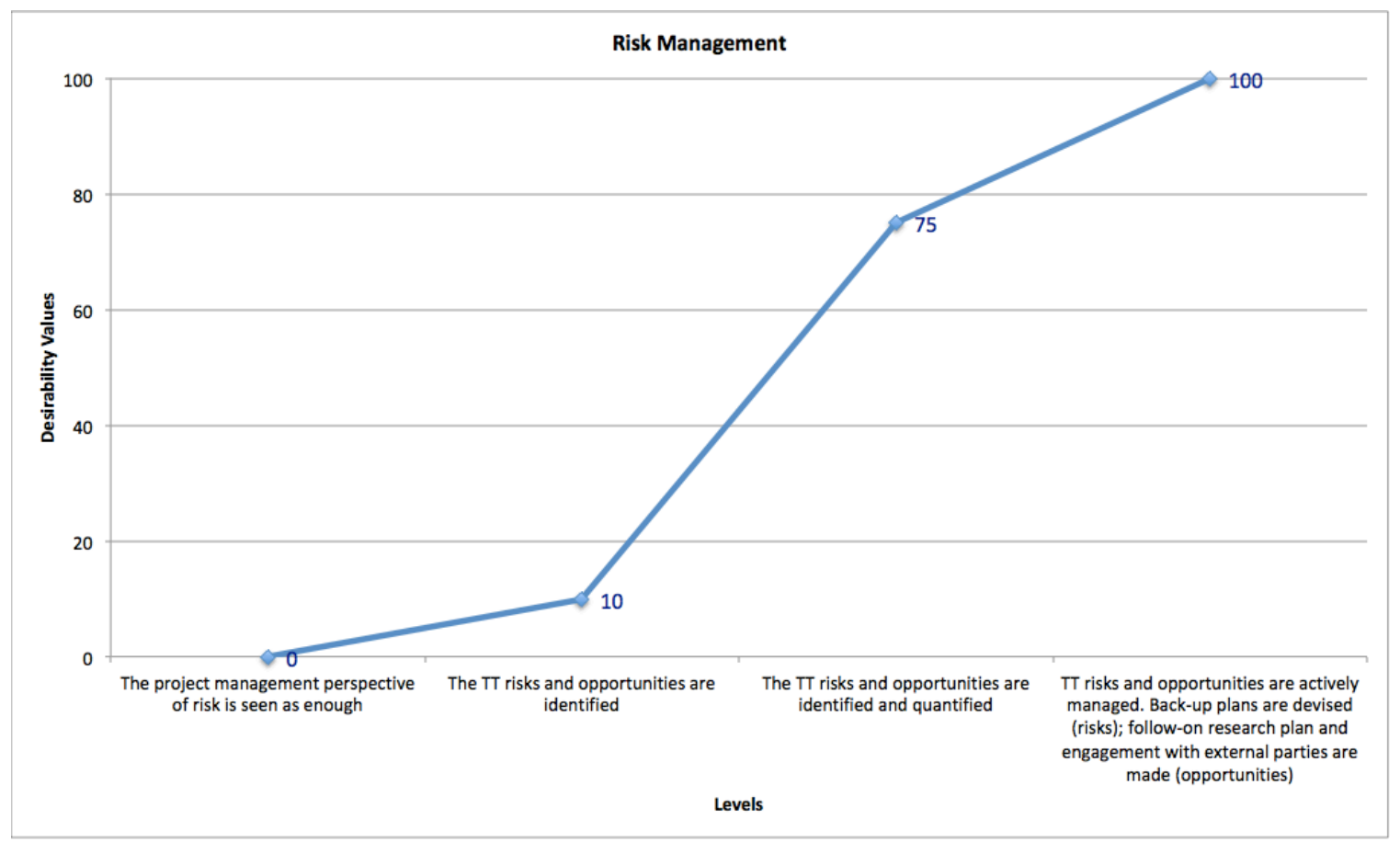

Figure 37 - Risk Management Curve

\section{Outcomes and decisions}

- No reports are produced, and no decisions are made out of the TT process.

- Non-standardized reports are produced, and recommendations are made based on individual's "gut-feeling".

- Standardized reports are produced for every project; Go-no go decisions are made based on TT data.

- Project managers and portfolio managers consult with the TT team, and use TT data before any major decision.

- TT Team approval is required for every major decision on technology development projects. 


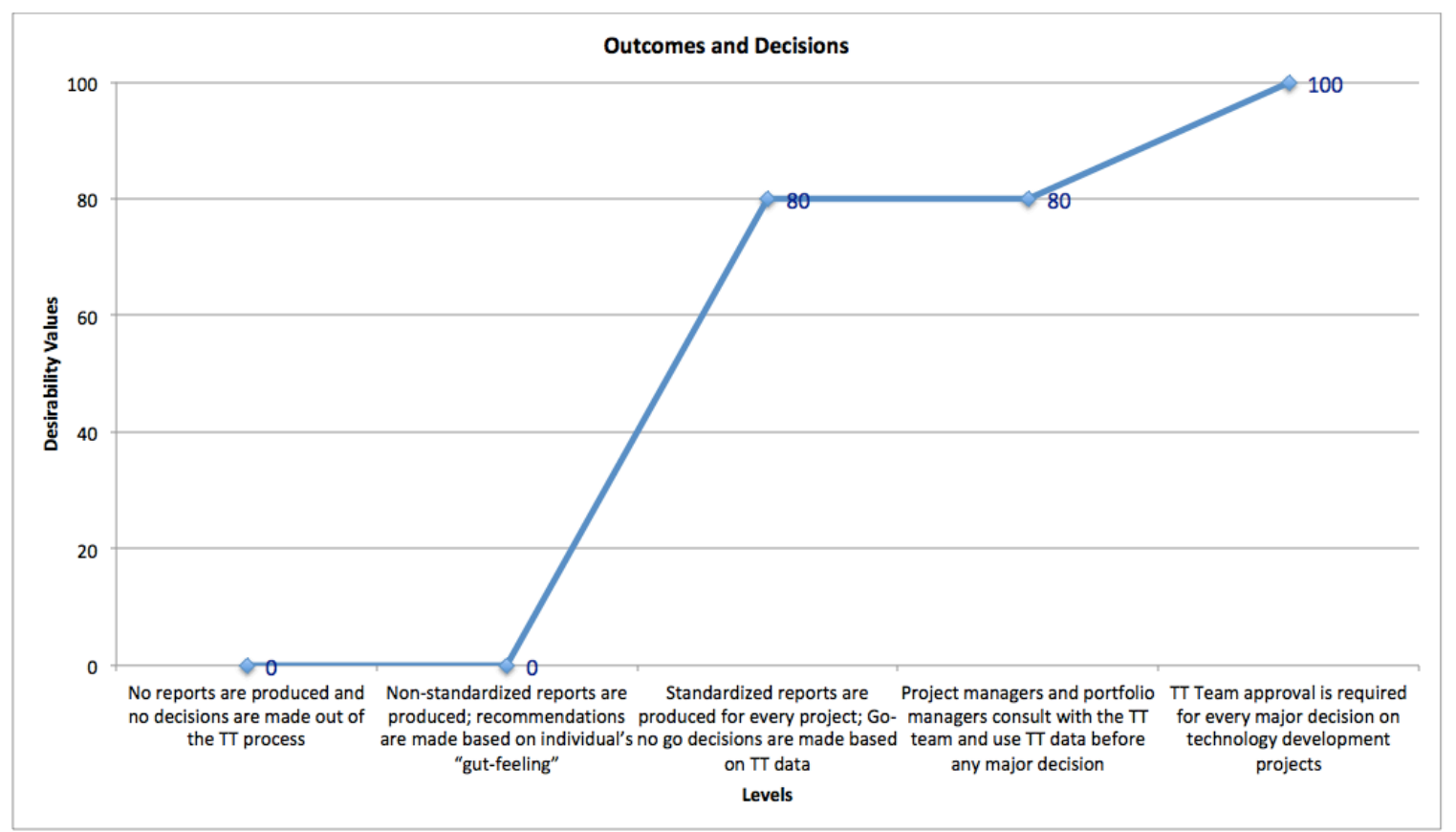

Figure 38 - Outcomes and Decisions Curve

\section{TT Mechanisms Management}

- Mechanisms are not identified or planned for before the technology is ready.

- For each project, one TT mechanism is chosen ahead of time and a plan based on the mechanism is devised.

- For each project, two TT mechanisms are chosen ahead of time and a plan for each mechanism is devised.

- For each project, three TT mechanisms are chosen ahead of time and a plan for each mechanism is devised.

- For each project, more than three TT mechanisms are chosen ahead of time and a plan for each mechanism is devised. 


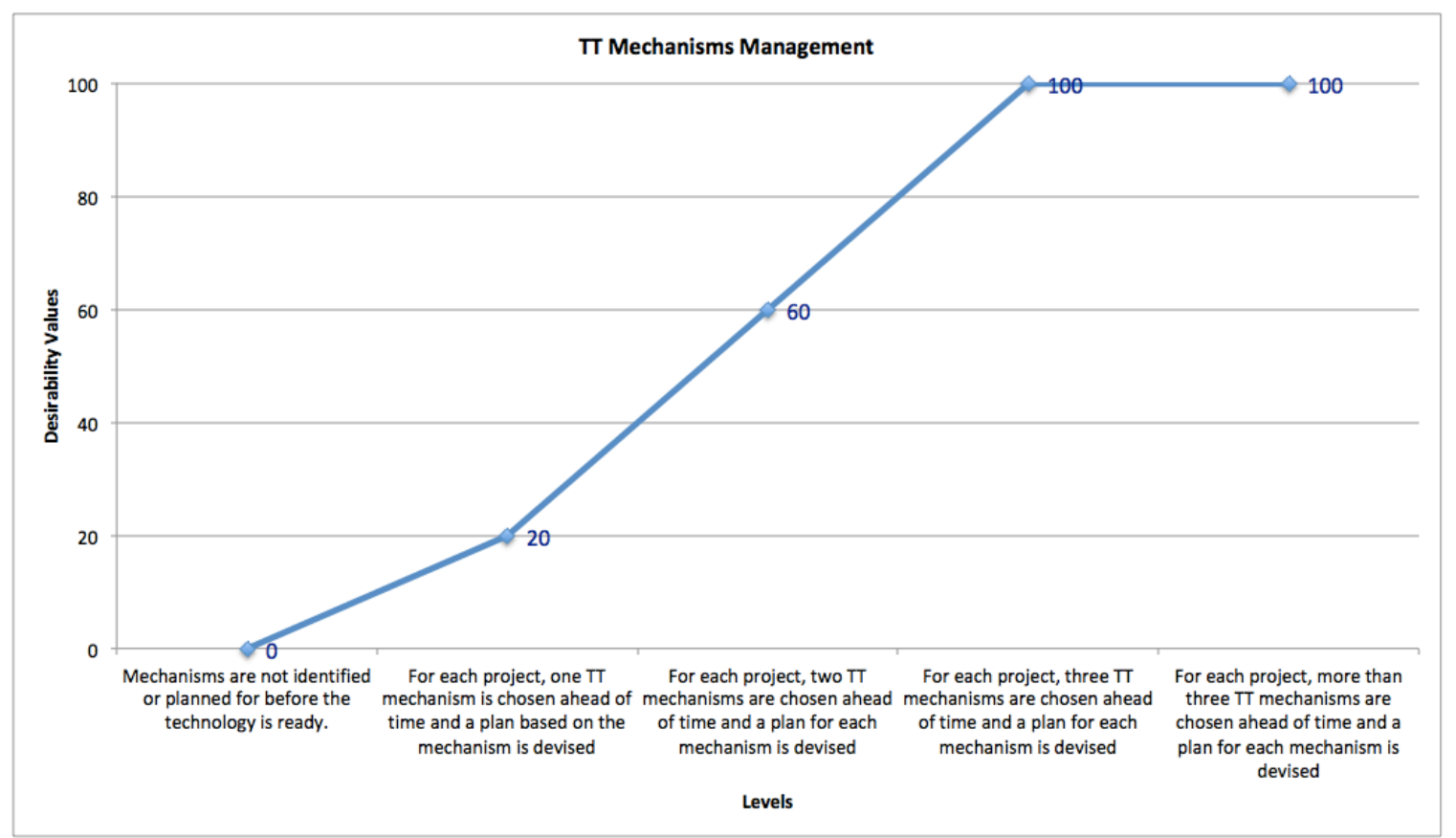

Figure 39 - TT Mechanisms Management Curve

\section{Strategic Alignment Perspective}

\section{Value, Impact and Applicability Management}

- No assessment is made throughout the technology development cycle.

- One assessment is made - at project start.

- Two assessments are made - at project start and end.

- Three assessments are made - at project start, mid-point and end.

- Assessments are dynamically made based on changes regarding the project, technology, market, and corporate strategy 


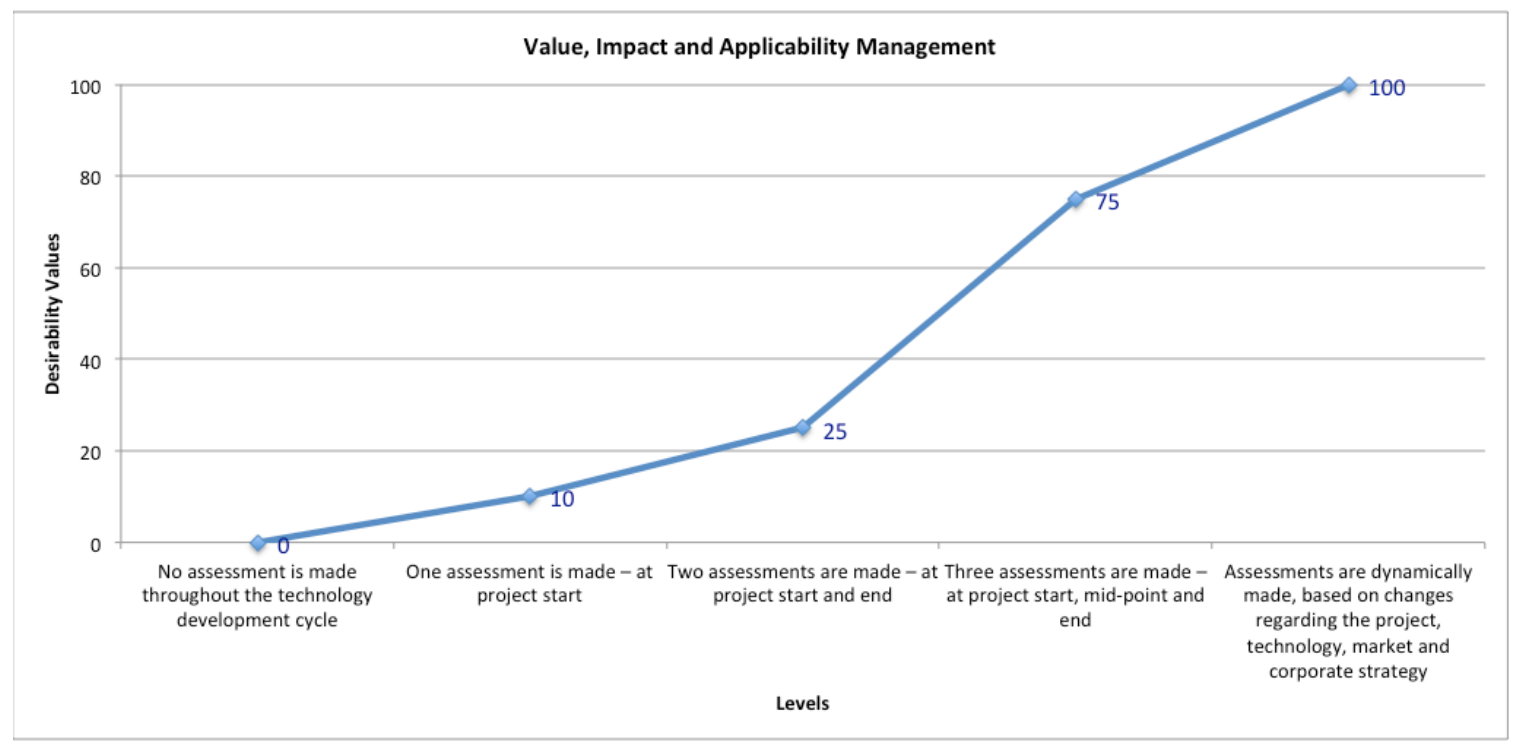

Figure 40 - Value, Impact and Applicability Management Curve

\section{Business Plan and Use Case}

- The organization does not create use cases for its technologies.

- Uses cases are available, but it only has little of the required information.

- Uses cases are available, but it only has some of the required information.

- Uses cases are available, and it has most of the required information.

- Uses cases are available, and it has all the required information. 


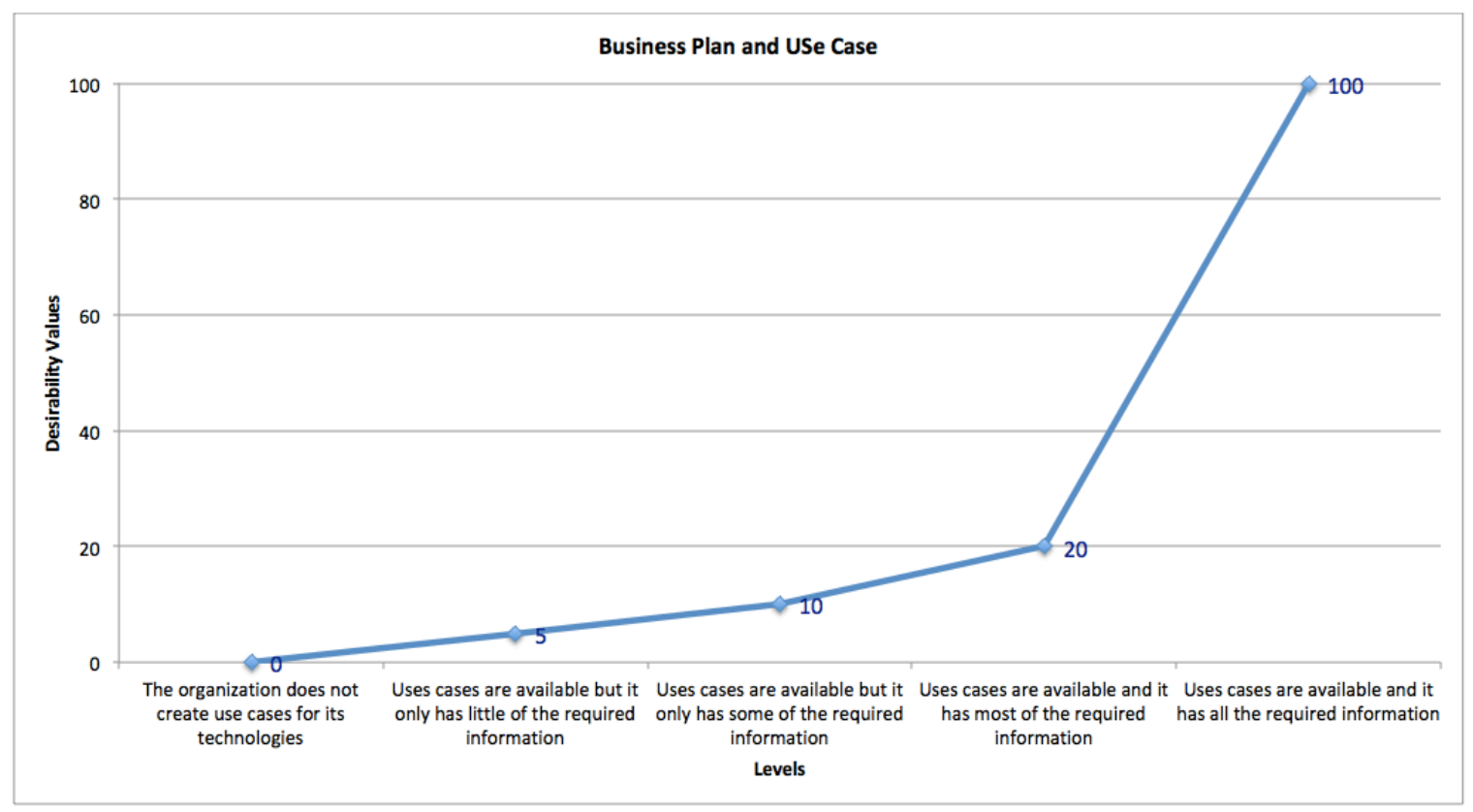

Figure 41 - Business Plan and Use Case Curve

\section{TT Planning, Control and Flexibility}

- No review and control efforts are done.

- Annual meetings are conducted to review the process against strategic management guidelines, to set priorities and objectives, and to identify and reflect on "lessons-learned".

- Quarterly meetings are conducted to review the process against strategic management guidelines, to set priorities and objectives, and to identify and reflect on "lessons-learned".

- Monthly meetings are conducted to review the process against strategic management guidelines, set priorities and objectives, and to identify and reflect on "lessons-learned". 
- Weekly meetings are conducted to review the process against strategic management guidelines, to set priorities and objectives, and to identify and reflect on "lessons-learned".

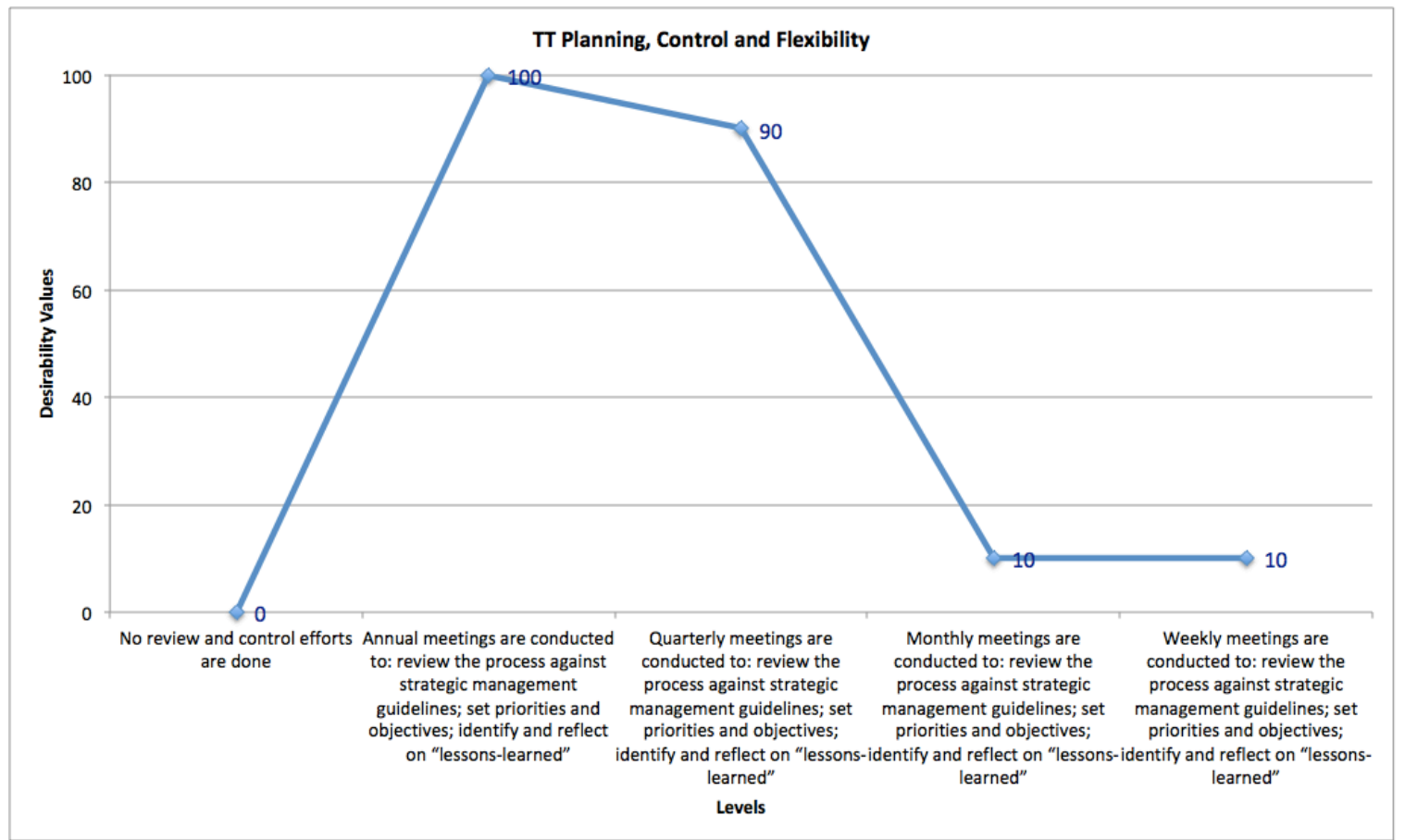

Figure 42 - TT Planning, Control and Flexibility Curve

\section{Continuity of TT Process}

- $\quad$ TT process starts after the technology development project is finished.

- TT process starts at very late stages of the technology development project.

- TT process starts around the mid-point of the technology development project.

- TT process starts at very early stages of the technology development project. 
TT process starts in the proposals and ideas assessment phase even before the technology development project

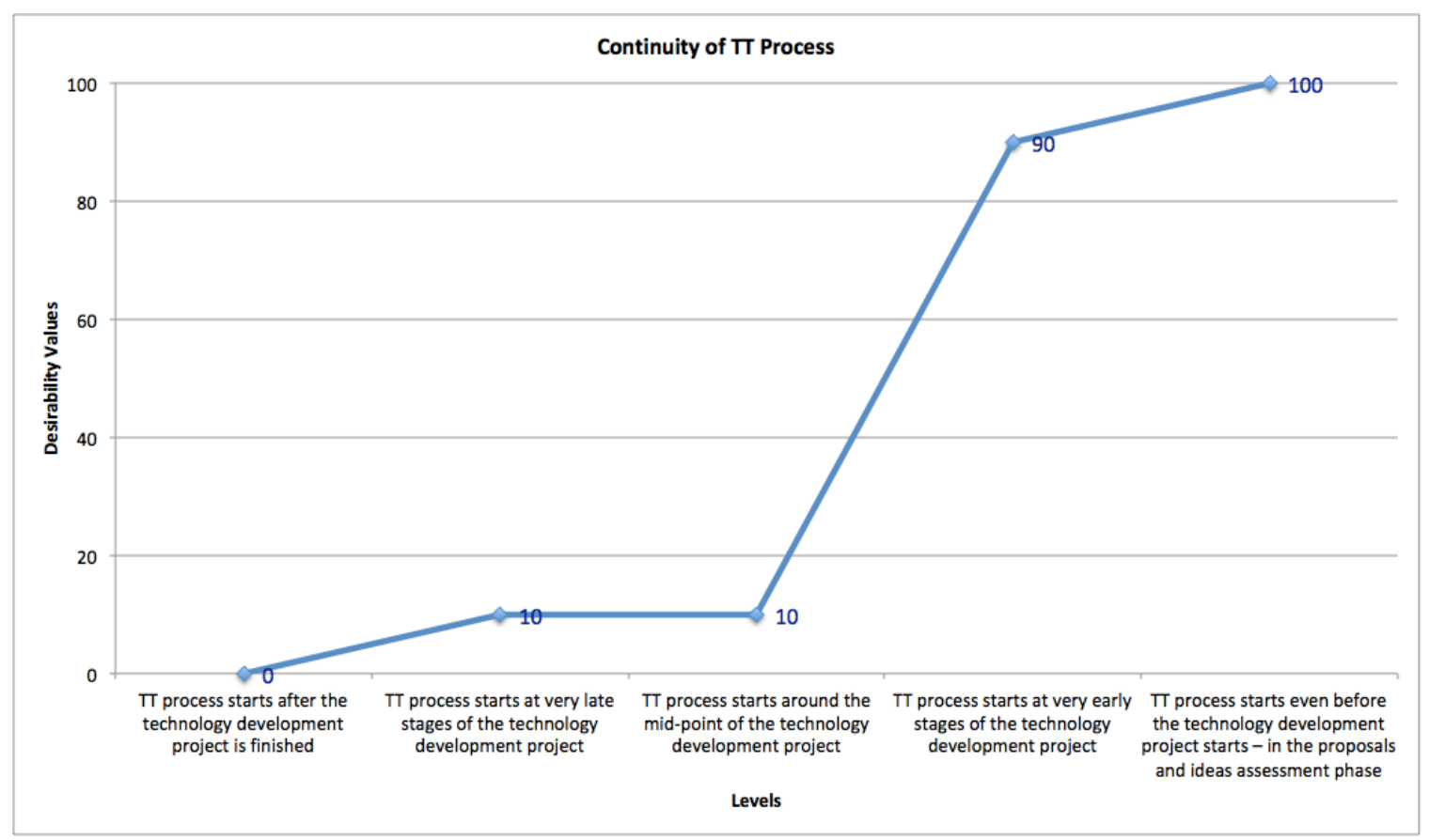

Figure 43 - Continuity of TT Process Curve

\section{Parallel Processes Integration}

- TT process is conducted completely isolated from other managerial processes.

- TT process exchanges information with project management; stage-gates decisions take the TT perspective into account.

- TT process exchanges information with project management and roadmapping; stage-gates decisions take the TT perspective into account; and roadmaps are changed to reflect TT transfer results/achievements.

- TT process exchanges information with project management, roadmapping and portfolio management; stage-gates decisions take the TT perspective 
into account; roadmaps are changed to reflect TT transfer

results/achievements; portfolio management receives input from the TT

process, aiming for a balanced TT portfolio (in terms of mechanisms, time and resources).

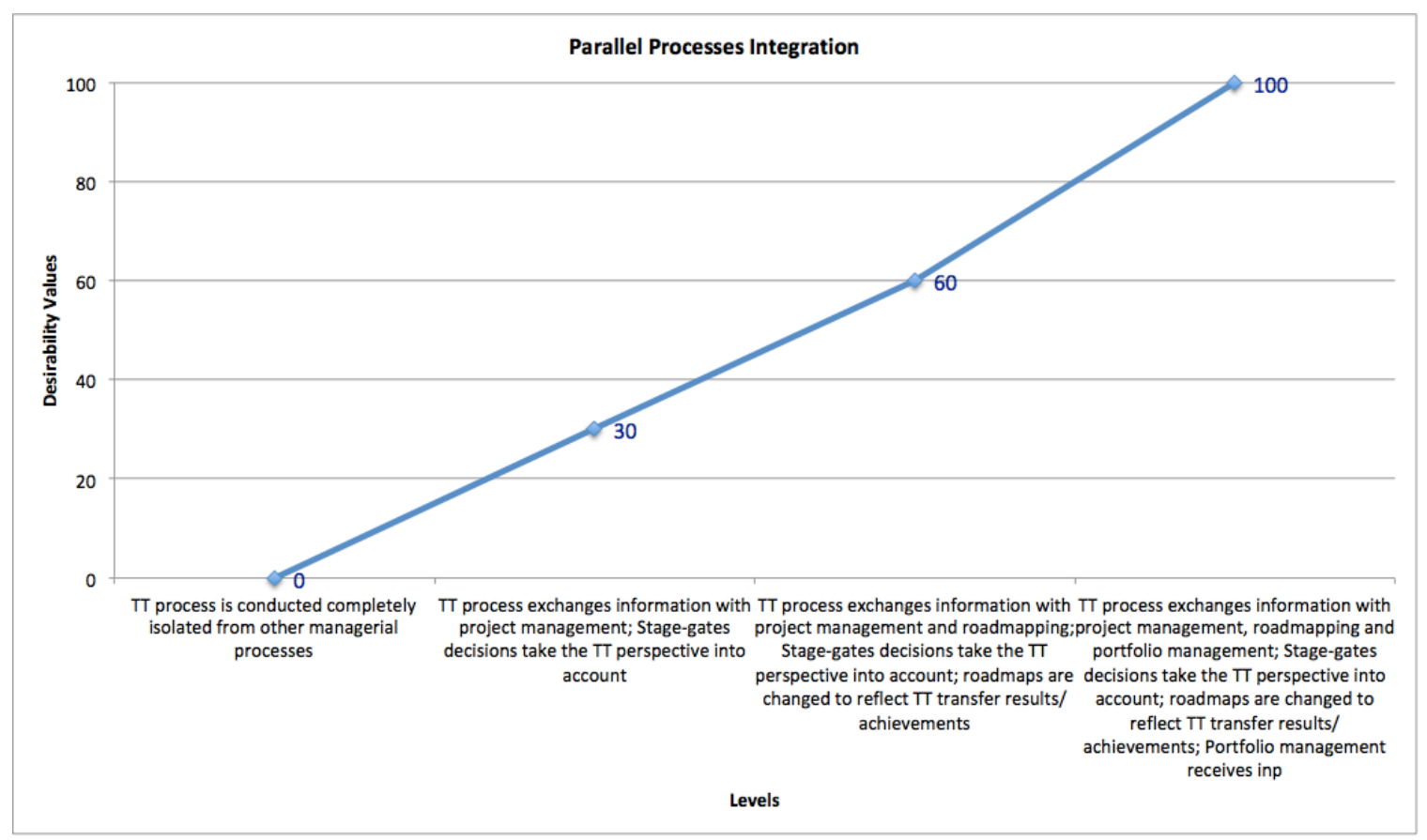

Figure 44 - Parallel Processes Integration Curve 


\section{CHAPTER 5 - RESEARCH MODEL APPLICATION}

The model developed in this research was applied in the Northwest region of the United States. More specifically, Bonneville Power Administration (BPA) was the organization assessed throughout the development of this study. The action research project that preceded this study and aimed at enhancing the agency's technology transfer process lasted for approximately two years. In this section, a brief discussion of BPA's TT process is presented, as well as a presentation on how the action research project unfolded. Lastly, the results of the model application and the sensitivity analysis are presented.

\section{BPA's TT process}

The project started in mid-2015, and aimed to create a more formal process / framework to enhance the agency's technology transfer capabilities, and hence improve their technology development results as a whole. The objective of the process was to create the conditions for the technology recipients (users) to be able to use the outcomes of the $R \& D$ projects to their full extent, and to maximize the benefits to the agency. These conditions would, ideally, ease the transfer transition process between donors and recipients, and to minimize costs, time, and effort for the outcomes to be used.

In mid-2017, roughly two years after the first efforts started, the core objective of the project remained the same, i.e. to create the conditions that will allow technology innovation projects' outcomes to flourish and yield the full extent of their benefits, in a way as to minimize the burden to the agency in terms of cost, time, and 
effort expended during the transition period between finishing the project and starting to use its outcomes. There was, however, a different nuance in the way the project was being directed. Back in 2015, the efforts were mainly towards understanding the best way to create a framework, and to figure out what components, steps, data, analyses, and people would be required to put the framework together. In 2017, a general framework had been created and therefore the efforts were more directed towards testing and refining the framework, and also understanding how to translate the framework application into solid actions to the benefit of the agency. Specifically, BPA's TT efforts have four objectives: The direct application of technology innovation outcomes to BPA to benefit the agency and regional stakeholders; to disseminate knowledge and research results in a way that maximizes the value of R\&D investments for the BPA business; to capitalize on the BPA's technological capability and knowledge; and to contribute to the utility industry and the region by sharing BPA's innovative technologies and applications.

\section{BPA's TT Process in Light of Literature}

The first observation to be made when contrasting the literature on technology transfer/commercialization and BPA's process is that the idea of commercializing products coming out of $\mathrm{R} \& \mathrm{D}$ projects is not completely applicable to all BPA's projects. As already discussed, the concepts of technology transfer and technology commercialization are very much intertwined (and were used interchangeably throughout this text). Nonetheless, technology transfer would be broader, in the sense of moving a technology from context A to context B, regardless of the purposes 
of the moving, whereas technology commercialization carries a strong market perspective to it, in the sense of bringing new technologies and products to consumers [103].

Looking at BPA's research portfolio, it is clear that projects related to the demand-response (DR) and the energy efficiency (EE) areas are a perfect fit for the technology commercialization concept. These are projects closely related to the final end of the energy sector spectrum, i.e. distribution utilities and residential/industrial/commercial consumers. Examples of end-products for those projects would be DR communication devices to be attached to home appliances; efficient lighting systems operated by sensors for industrial and commercial parking lots; and energy efficient appliances such as combined space and water heating heat pump systems for residential users. The ultimate objective of these projects is for products and systems to be adopted in the market, e.g. appliance manufacturers to adopt the DR devices; distribution utilities to adopt DR communication standards; grocery stores to adopt efficient lighting systems in their parking lots; and consumers to purchase heat-pump systems instead of resistive systems. Therefore, technology commercialization is a good term to represent their transfer efforts. Nonetheless, a significant part of BPA's R\&D portfolio is composed of power generation and transmission projects. These, as opposed to the EE and DR ones, are almost exclusively focused on BPA's internal operations and needs, and the concern about its outcomes being adopted beyond BPA's boundaries is limited, if not absent altogether. Examples of end products for those projects would be new coating materials and processes for hardening the surface of turbine blades in hydroelectric power plants, 
and sophisticated software frameworks to interpret and analyze phasor measurement units (PMU) data from transmission lines. The ultimate objective of these projects is for power generation and transmission to be more reliable and available, regardless of if other utilities would or would not adopt their outcomes. To exemplify the difference in nature of these areas, let us think of two imaginary projects: The development of a super-efficient clothes dryer and the development of an earthquake-resilient substation design. The former has a very strong market perspective to it, while the latter does not. The clothes dryer development might be a success from a technical standpoint, but if it does not match the requirements and expectations of both consumers and appliance manufacturers, it will not be a successful project. Often these projects are conducted in partnership with external parties that coordinate market transformation and technology adoption approaches, (the Northwest Energy Efficiency Alliance (NEEA), for example), approaches without which those projects become useless. The substation design, on the other hand, is developed exclusively with BPA's requirements in mind. If the design achieves the earthquake resilience level it was meant to, it is a successful project. If other entities, such as the Western Area Power Administration (WAPA), are not interested in the outcomes of the project, or even if consumers do not see enough value in it, BPA is likely to continue the project nonetheless, as it would bring strategic benefits to the agency. Cao et al. suggest that due to several obstacles, EE technologies still need to have public policies as a major ally to boost its adoption and dissemination [125]. EE is really a different "animal", and we see that at BPA. Concepts, requirements, and approaches that work with transmission and generation technologies not always are 
applicable to EE technologies. BPA's TT team has already identified as one of its biggest challenges the need to adapt the TT process, and the way we assess and approach the projects towards EE needs. Especially regarding benefits, EE is very distinct from other energy technologies, even more so in the case of BPA, who does not serve the final consumer directly.

Another discrepancy between what the literature says and BPA's process is the intellectual property issue. As previously mentioned, IP rights are a main concern in the technology transfer literature. Due to the fact that BPA is a federal non-profit organization, its main R\&D objective is to benefit the whole energy sector and final consumers, thus it is not a concern if other stakeholders hold the IP rights and use it, which is actually desirable. In the case of private companies, patents are very important as a means to maximize profits and market-share by imposing market entry barriers and minimizing competition. BPA, however, aims to spread out its R\&D outcomes as much as possible. Although IP rights are a concern for BPA, it is not the strongest one. The flipside would be the regulatory issues. While private companies might see regulations as a secondary issue when conducting R\&D, BPA is heavily limited by regulations and standards.

As already shown, the literature discusses the necessity of having technology transfer as a part of the research and development strategy [5]. Before the TT project started, this was not a reality at BPA. Technology transfer was not seen as an important component in the R\&D management framework, but rather as a small part of project management efforts. With the work developed throughout the years, this notion has been changed, and now TT is regarded as an important part of R\&D. 
Technology transfer concerns are starting to play a major role in the way the agency manages its R\&D projects. Another important improvement point brought by the literature, and that is being developed, is the partnership synergy. Scholars suggest that synergy between R\&D partners is essential for energy technology development [114]. Partnerships existed in BPA's R\&D long before the technology transfer project started, but it is only lately that a more meticulous assessment of the partnerships is being discussed. Although this is not implemented yet, some of the new information required for proponents to provide is an analysis of project stakeholders, aiming to better understand the agency's partners.

Although BPA's technology transfer process has improved significantly since the start of the project, some of the practices indicated in the literature towards a more successful technology commercialization were already present in the agency. For instance, the need to have both internal and external technology development efforts is mentioned as a way to balance the research portfolio and to mitigate risks involved in the process [104]. BPA has had, before the start of the technology transfer project, both types of R\&D projects. Additionally, scholars also mention that partnering with research universities is essential for developing new energy technologies [115], and BPA already had joint development projects with universities all across America, even before the technology transfer project started.

\section{The Action Research Project}

As stated by [156], the four building blocks or steps of an action research project are the following: 
- To create communities of inquiry within communities of practice, shortening the distance, and making no difference between scientists and practitioners.

- To build theories in practice by going to the practical sphere to build theory.

- To combine interpretation with testing by testing the theory with practical applications.

- To change the status quo, and causing actual changes to the practitioner's systems.

If a given project follows these steps and has these components, it can be confidently characterized as an action research project. As already discussed, BPA's technology transfer project has made the agency's TT framework to evolve overtime. The process through which the framework has evolved, as explained earlier, has gone through all four steps of an action research project, as explained in the next paragraphs.

The research team was actively involved in the project, not only as observers but as members of the team, engaging in conversations, participating in discussions, and meetings and bringing ideas, concepts, and insights to the table. This active participation remained until the project ended, perfectly characterizing the first step of AR. Moreover, a fair share of the project's first step was devoted for the researchers to understand and familiarize themselves with the practitioners' systems, processes, and environments. Clearly, there was no difference between researchers and practitioners. 
Also, during the first months of the project, the researchers were diving into the literature looking for and analyzing technology transfer frameworks. The objective of this task was for the researchers to present the theoretical models and frameworks, discuss them with the team, compare these theoretical frameworks with the current practice the agency had, and to propose changes and enhancements. Several rounds of discussion were conducted where the researchers would acquire information and share with the entire team; the team (researchers included) would discuss the ideas, and contrast them with the current system and propose changes; the researchers would then go back to the literature and re-start the cycle. This entirely satisfies the second step of AR where researchers have to go back and forth between theory and practice, engaging practitioners in discussions, and proposing changes to their systems. As an example, one could mention the creation of technology transfer evaluation points. These were forms developed based on a comprehensive pool of criteria and perspectives present in the literature, and brought to the team by the researchers (several iterative cycles were undertaken). Both the application timing and criteria to be used in each form were extensively discussed, with active participation from both practitioners and researchers.

Throughout the project, whenever an idea was being developed, there were several iterative cycles of discussion before revisions were made. After the result was considered acceptable, the team would pilot that result with project managers and other stakeholders. This fits perfectly into the third AR step definition. In action research projects, researchers are not only prompted to suggest changes to practitioners' systems, but also to test those changes. 
The fourth and last step of an action research project requires researchers to go beyond everything they did in the first three steps. After actively participating in discussions and understanding the practitioners' systems, having gone back and forth between theory and practice suggesting changes, and having tested those changes, the researchers have to implement those changes and analyze the results, going back to the first step afterwards, if necessary.

Additionally, it is possible to zoom out a bit and analyze the project unfolding from a higher level. The first phase of the project was one of an exploratory nature, when the researchers were discussing the practitioners' systems and understanding it in a very active fashion, thus creating a community of inquiry within a community of practice, shortening the distance between researchers and practitioners. The next step was to analyze both literature and BPA's processes, suggesting changes and enhancements to the process, and then publish a journal article about this experience that [277] focused on building theory in practice by going to the practical sphere to build theory). Next came the testing and implementation of the proposed changes and enhancements. This is where we test the theory with practical applications, and cause actual changes to the practitioners' systems).

\section{Application Results}

In order to apply the model to BPA, each factor desirability levels were contrasted against the agency's technology transfer process current status, aiming to assign the desirability values. The assignment was done based on a workshop conducted with key stakeholders in the agency. After assigning the desirability value 
of each factor concerning BPA's TT process, the aforementioned Organizational Technology Transfer Score formula was applied, thus arriving at BPA's TT score.

Following is the desirability values assignment for BPA:

\section{HR and Stakeholders Perspective}

- TT team and training:

○ Assigned level: Dedicated TT team; not multidisciplinary; no training.

- Desirability value: 10

- Reasoning: Although there is a team, it is comprised of representatives from only one department, the TI Office; no technology transfer training exists.

- TT ecosystem management:

- Assigned level: Frequent interaction (at least monthly) with unstructured ad-hoc discussions.

○ Desirability value: 60

- Reasoning: BPA maintains close relationship with $3^{\text {rd }}$ parties, especially National Labs and research centers around the country.

- Stakeholders management:

- Assigned level: TT team has frequent contact (at least monthly) with internal stakeholders (and external, if any); information goes one way (from the stakeholders, but with solicitation).

○ Desirability value: 20 
O Reasoning: BPA TT team has frequent contact with internal stakeholders, both recipients and donors; Information is collected from these stakeholders, but the flow of information is not constant yet, i.e. most of the information collected has to be solicited.

- Senior management involvement:

○ Assigned level: Middle management is actively engaged in the TT process; Senior management is aware of the TT process, but their engagement is not consistent.

○ Desirability value: 70

○ Reasoning: Middle management is consistently supportive and involved in the process development (CTO); Senior managers, in spite of being aware of the efforts, could support, engage in discussions, and participate in a more active way, as to strategically guide the team.

\section{Organizational Culture Perspective}

- Communication and knowledge management:

○ Assigned level: Inter-departmental communication is weak; only vital information is transferred from one department to another without solicitation.

○ Desirability value: 10

○ Reasoning: The flow of information between groups could and should be better and more intense. Although some progress seems to be happening, this level still best describes the current situation. 
- Funding:

○ Assigned level: No budget is allocated.

○ Desirability value: 0

- Reasoning: No budget is constantly allocated towards TT activities; some investments have been made in some instances, but this occurs on a case-by-case basis.

- Absorptive capacity:

- Assigned level: The organization creates steady and successful partnerships, collaborative development, or joint R\&D efforts. These amount to $25 \%$ to $50 \%$ of its research portfolio.

○ Desirability value: 100

○ Reasoning: BPA has several ongoing partnerships with different entities across the US.

- Long-range planning:

- Assigned level: Formal corporate and business strategies exist, but are tacitly propagated.

- Desirability value: 20

○ Reasoning: Although there is a formal strategy in place, different groups and individuals are still trying to grasp it and to understand how to adapt their daily activities to fit the strategic planning.

- Innovative Culture: 
- Assigned level: Minor innovations / changes in business practices have been successfully implemented in the last three years.

○ Desirability value: 60

- Reasoning: Minor changes in how groups operate have happened, but are not agency-wide.

\section{Technical Perspective}

- TRL Assessment

- Assigned level: Technology readiness is assessed, and it informs the decision-making process regarding TT mechanisms and strategies.

○ Desirability value: 50

- Reasoning: TRL assessment is conducted, and it informs the transfer preparation. For instance, if the final product is at TRL 9, supply chain and procurement processes are initiated; if the final product is at TRL 5, a follow-on research is contemplated. However, no additional measurements are made.

- Technology valuation:

- Assigned level: One technology assessment study is conducted at the beginning of the technology development cycle.

- Desirability value: 10

○ Reasoning: The only technology assessment-like effort is conducted by ways of analyzing the research proposal, and the information therein explained. 
- Proposal assessment:

○ Assigned level: Research proposals are not methodically assessed.

○ Desirability value: 0

- Reasoning: Although there used to be a formalized process in place to evaluate and score proposals, it has been substituted by a more informal and subjective evaluation.

\section{Process Perspective}

- Benefit management:

- Assigned level: Benefits are differentiated from project deliverables, classified and quantified, without stakeholders' input or program considerations.

- Desirability value: 60

- Reasoning: One of the technology transfer forms' objectives is to classify and quantify the expected benefits deriving from each project, but these assessments are conducted only as long as the project is still active. There is no post-project effort as of now. Moreover, a programmatic approach is not explicitly taken while evaluating benefits.

- Risk management:

- Assigned level: The TT risks and opportunities are identified.

○ Desirability value: 10 
○ Reasoning: TT forms also aim to identify risks and potential opportunities, but no quantification is performed, and no backup plans are devised.

- Outcomes and decisions:

- Assigned level: Non-standardized reports are produced; recommendations are made based on individual's "gut-feeling".

- Desirability value: 0

- Reasoning: A standardized TT report for each project has not been developed; go-no-go decisions are only made based on the project management dimension.

- TT mechanisms management:

- Assigned level: For each project, one TT mechanism is chosen ahead of time and a plan based on the mechanism is devised.

○ Desirability value: 20

- Reasoning: One and only one TT mechanism is expected per project, and no backup plans are made ahead of time.

\section{Strategic Alignment Perspective}

- Value, impact, and applicability management:

- Assigned level: One assessment is made at the project start.

○ Desirability value: 10

- Reasoning: These perspectives are formally assessed only once, before funding is awarded to projects. 
- Business plan and use case:

- Assigned level: Uses cases are available, and they are most of the required information.

○ Desirability value: 20

- Reasoning: For most of the projects, a clear and comprehensive use case is created to inform stakeholders on how the outcomes are expected to be used.

- TT planning, control and flexibility:

- Assigned level: Annual meetings are conducted to review the process against strategic management guidelines; to set priorities and objectives; and to identify and reflect on "lessons-learned".

○ Desirability value: 100

- Reasoning: Although not specific to TT, reviews exist about the whole Technology Innovation Office processes.

- Continuity of TT process:

- Assigned level: TT process starts in the proposals and ideas assessment phase even before the technology development project starts.

○ Desirability value: 100

- Reasoning: TT efforts are conducted before any investments are made, with the benefit form being filled out and analyzed during the proposal evaluation phase. 
- Parallel processes integration:

- Assigned level: TT process exchanges information with project management; stage-gates decisions take the TT perspective into account.

- Desirability value: 30

- Reasoning: The TT process exchanges information with other processes (most importantly project management), but the process outcomes are not actively being used by other processes. Currently, an effort aimed at informing the roadmapping process and updating the roadmaps based on the outcomes of the projects and information coming from the TT process is being discussed, but has not been implemented yet.

Table 57 summarizes the assignment of values from the desirability curves to BPA's TT process.

Table 57 - Summary of DC Values Assignment to BPA

\begin{tabular}{|c|c|c|}
\hline \multirow{3}{*}{ Perspective } & Factor & $\begin{array}{c}\text { Value from DC assigned to } \\
\text { BPA }\end{array}$ \\
\hline \multirow{4}{*}{ HR and Stakeholders } & TT team and training & 10 \\
\cline { 2 - 3 } & TT ecosystem mgmt.. & 60 \\
\cline { 2 - 3 } & Stakeholders mgmt. & 20 \\
\cline { 2 - 3 } & Senior mgmt. involvement & 70 \\
\hline \multirow{3}{*}{ Organizational Culture } & Communication and knowledge mgmt. & 10 \\
\cline { 2 - 3 } & Funding & 100 \\
\cline { 2 - 3 } & Absorptive capacity & 0 \\
\cline { 2 - 3 } & &
\end{tabular}




\begin{tabular}{|c|c|c|}
\hline \multirow{4}{*}{ Technical } & Long-range planning & 20 \\
\cline { 2 - 3 } & Innovative Culture & 60 \\
\hline \multirow{4}{*}{ Process } & TRL assessment & 50 \\
\cline { 2 - 3 } & Technology valuation & 10 \\
\cline { 2 - 3 } & Proposal assessment & 0 \\
\cline { 2 - 3 } & Benefit mgmt. & 60 \\
\cline { 2 - 3 } & Risk mgmt. & 10 \\
\cline { 2 - 3 } & Outcomes and decisions & 0 \\
\hline \multirow{5}{*}{ Strategic Alignment } & TT mechanisms mgmt. & 20 \\
\cline { 2 - 3 } & Value, impact and applicability mgmt. & 10 \\
\cline { 2 - 3 } & Business plan and use case & 20 \\
\cline { 2 - 3 } & TT planning, control and flexibility & 100 \\
\cline { 2 - 3 } & Continuity of TT process & 100 \\
\cline { 2 - 3 } & Parallel processes integration & 30 \\
\hline
\end{tabular}

The computation of the Organizational Technology Transfer Score is done by the application of the formula presented earlier. Table 58 shows the results.

Table 58 - BPA's organizational TT Score

\begin{tabular}{|c|c|c|c|c|c|}
\hline \multirow{2}{*}{ Perspectives } & \multicolumn{2}{|l|}{ Factors } & \multicolumn{3}{|c|}{ TT Score } \\
\hline & Name & $\begin{array}{l}\text { Global } \\
\text { value }\end{array}$ & BPA values & $\begin{array}{l}\text { values } x \\
\text { factors }\end{array}$ & $\begin{array}{l}\text { Perspectives } \\
\text { sum }\end{array}$ \\
\hline \multirow{4}{*}{$\begin{array}{c}\text { HR and } \\
\text { Stakeholders }\end{array}$} & TT team and training & 0.07 & 10 & 0.7 & \multirow{4}{*}{7.7} \\
\hline & TT ecosystem mgmt. & 0.05 & 60 & 3 & \\
\hline & Stakeholders mgmt. & 0.06 & 20 & 1.2 & \\
\hline & $\begin{array}{c}\text { Senior mgmt. } \\
\text { involvement }\end{array}$ & 0.04 & 70 & 2.8 & \\
\hline \multirow{5}{*}{$\begin{array}{l}\text { Organizational } \\
\text { Culture }\end{array}$} & $\begin{array}{l}\text { Communication and } \\
\text { knowledge mgmt. }\end{array}$ & 0.05 & 10 & 0.5 & \multirow{5}{*}{6.5} \\
\hline & Funding & 0.03 & 0 & 0 & \\
\hline & Absorptive capacity & 0.03 & 100 & 3 & \\
\hline & Long-range planning & 0.03 & 20 & 0.6 & \\
\hline & Innovative Culture & 0.04 & 60 & 2.4 & \\
\hline \multirow{3}{*}{ Technical } & TRL assessment & 0.04 & 50 & 2 & \multirow{3}{*}{2.8} \\
\hline & Technology valuation & 0.08 & 10 & 0.8 & \\
\hline & Proposal assessment & 0.06 & 0 & 0 & \\
\hline
\end{tabular}




\begin{tabular}{|c|c|c|c|c|c|}
\hline \multirow{4}{*}{ Process } & Benefit mgmt. & 0.04 & 60 & 2.4 & \multirow{4}{*}{3.4} \\
\hline & Risk mgmt. & 0.04 & 10 & 0.4 & \\
\hline & $\begin{array}{c}\text { Outcomes and } \\
\text { decisions }\end{array}$ & 0.05 & 0 & 0 & \\
\hline & $\begin{array}{c}\text { TT mechanisms } \\
\text { mgmt. }\end{array}$ & 0.03 & 20 & 0.6 & \\
\hline \multirow{5}{*}{$\begin{array}{l}\text { Strategic } \\
\text { Alignment }\end{array}$} & $\begin{array}{l}\text { Value, impact and } \\
\text { applicability mgmt. }\end{array}$ & 0.07 & 10 & 0.7 & \multirow{5}{*}{11.7} \\
\hline & $\begin{array}{c}\text { Business plan and } \\
\text { use case }\end{array}$ & 0.04 & 20 & 0.8 & \\
\hline & $\begin{array}{l}\text { TT planning, control } \\
\text { and flexibility }\end{array}$ & 0.05 & 100 & 5 & \\
\hline & $\begin{array}{c}\text { Continuity of TT } \\
\text { process }\end{array}$ & 0.04 & 100 & 4 & \\
\hline & $\begin{array}{c}\text { Parallel processes } \\
\text { integration }\end{array}$ & 0.04 & 30 & 1.2 & \\
\hline & & & Total Sum: & 32.1 & \\
\hline
\end{tabular}

The final score shows that BPA is still far from having "perfect" technology score capabilities. Additionally, one could regard the final score as somewhat disappointing. It has been mentioned in this dissertation that the agency has been actively looking to increase its TT capabilities, including by means of an action research project in which I have myself participated. In order to avoid any misinterpretations regarding the agency's efforts and ability to improve, I find it important to make a few remarks. Firstly, the results of the model application show a snapshot at the time of the current status of BPA's TT capabilities, but tells nothing about its previous status or the improvements that have been made, and sharp improvements have been made. Secondly, several frameworks, tools, and processes that have been generated as part of the action research project have yet to be piloted and implemented, while some have been tested but not fully rolled out yet. It is my contention that once those are fully implemented into the agency's processes, that TT score is likely to increase in a significant fashion. 


\section{Sensitivity Analysis}

After obtaining the organizational technology transfer score for the Technology Innovation group at BPA, a sensitivity analysis is conducted to test the robustness of the model. This analysis aims to measure how the model's final output will react to changes in the relevance of criteria. In a model with different technology alternatives, for instance, the final ranking of the alternatives might be altered if the criteria relevance is altered. The sensitivity analysis would measure how strong or disruptive these changes would be. Bringing the same reasoning to the present model, the sensitivity analysis will indicate the changes in the final organizational TT score caused by alterations in the perspectives relevance. This could be particularly interesting if one is to use the model to compare different departments within an organization or to compare different organizations. Moreover, when applying the model to only one case (as in this study), changing the weights of the perspectives could prompt the technology manager to change his/her reasoning when prioritizing factors to be tackled. For example, if the technical perspective is, by far, the most relevant, weak factors under the technical perspective should be prioritized, even if the organization seems to be performing better in those factors relative to factors under other perspectives. Following sensitivity analyses made by Abotah and Estep [245], [263], different scenarios were created. Each scenario alters the relevance of perspectives by boosting one of the perspectives, i.e. on Scenario One, the perspective "HR and Stakeholders" has its relevance boosted to 0.96, while the remaining perspectives have theirs at 0.01 each, and so on. Following are the results of the five scenarios created. 
Table 59 - Sensitivity Analysis Scenario 1 Results

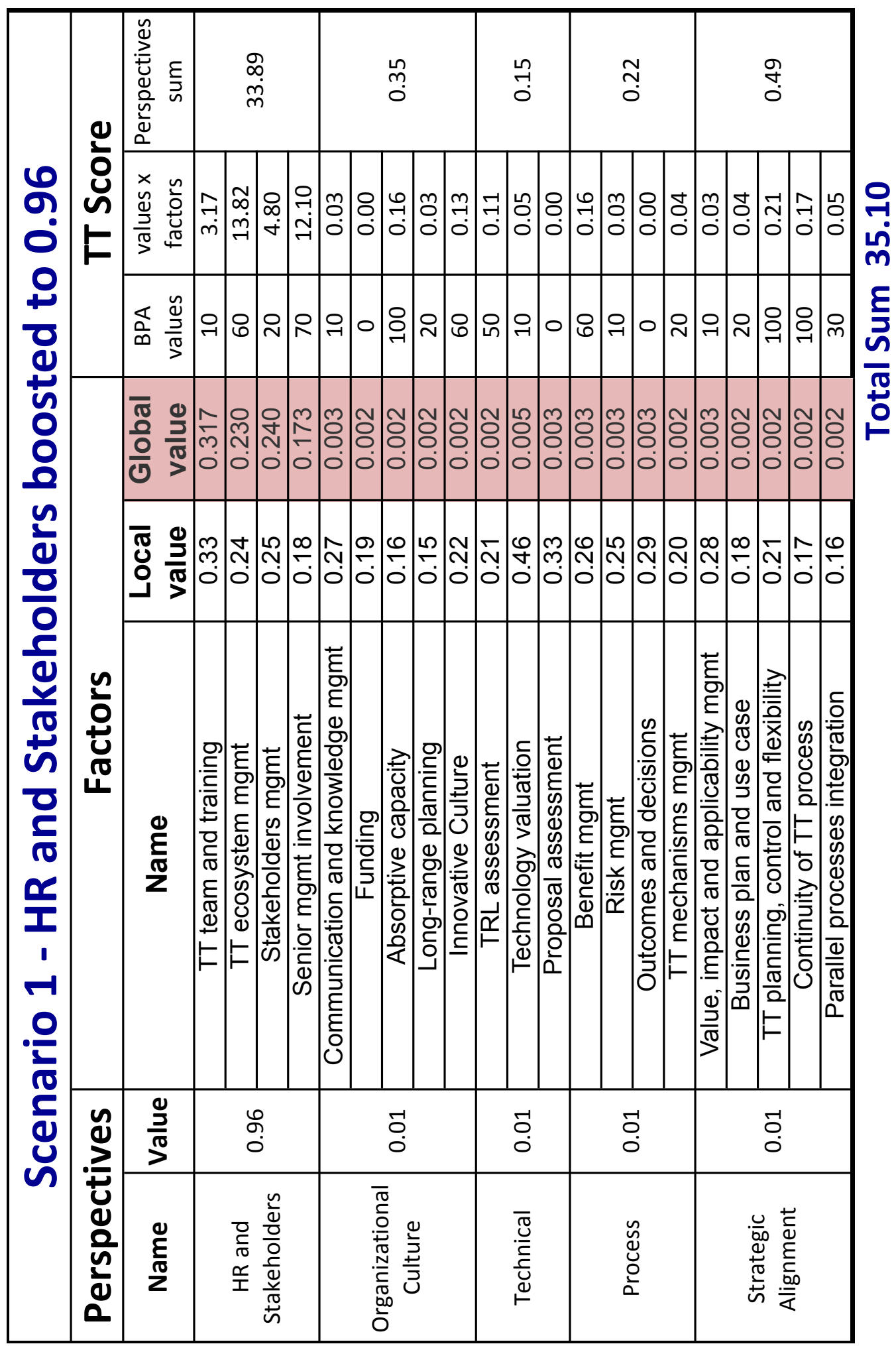


Table 60 - Sensitivity Analysis Scenario 2 Results

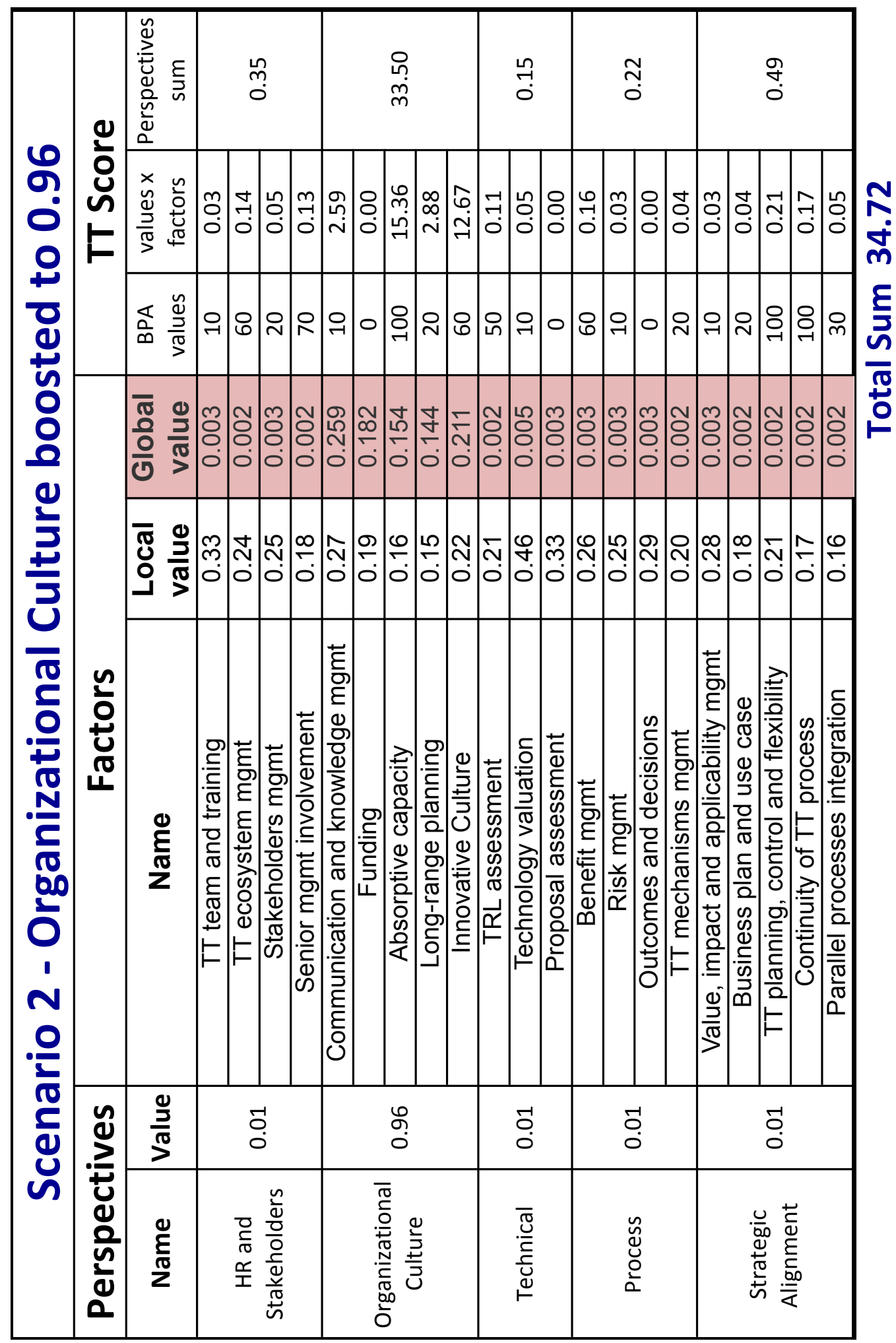


Table 61 - Sensitivity Analysis Scenario 3 Results

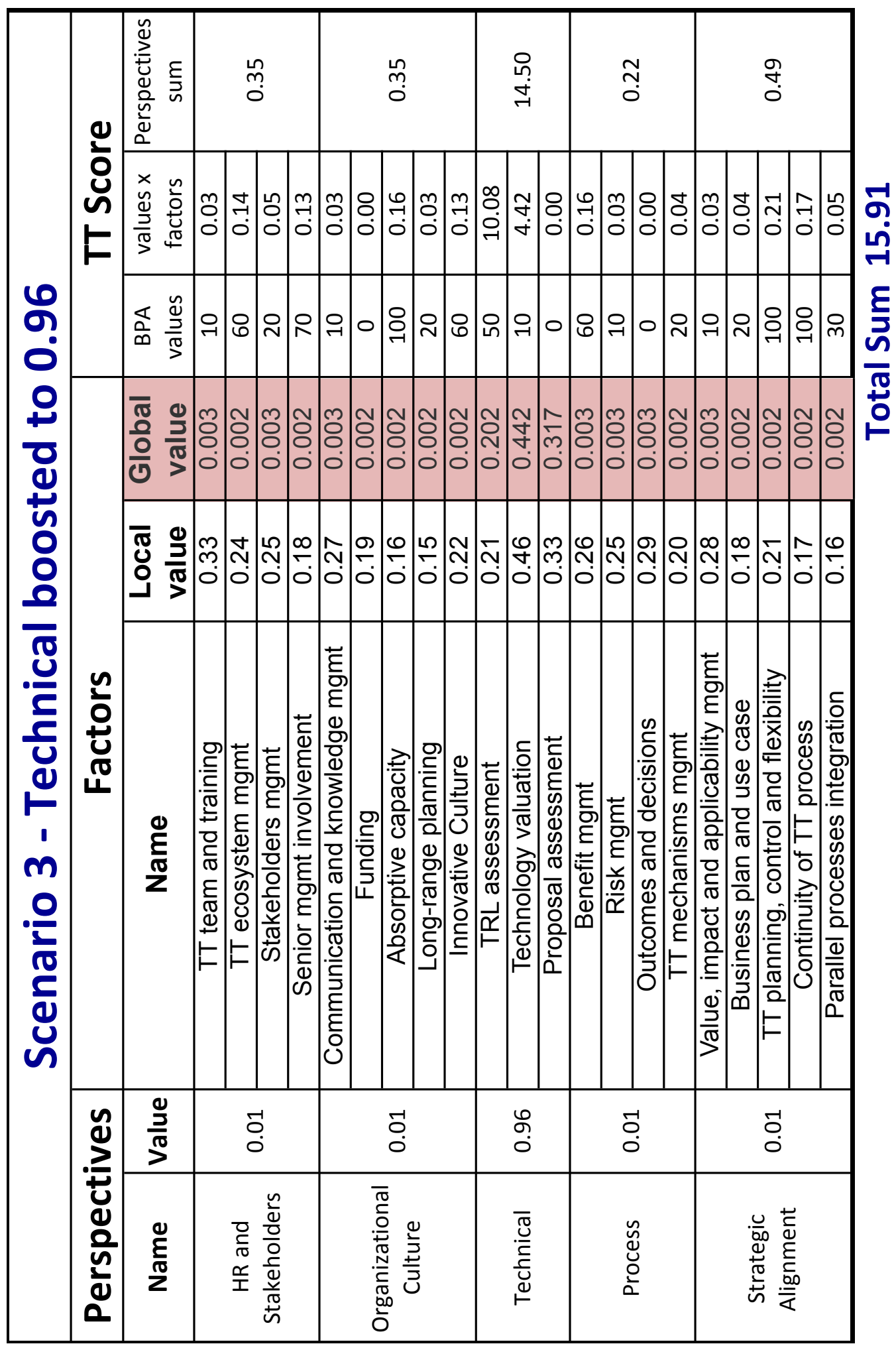


Table 62 - Sensitivity Analysis Scenario 4 Results

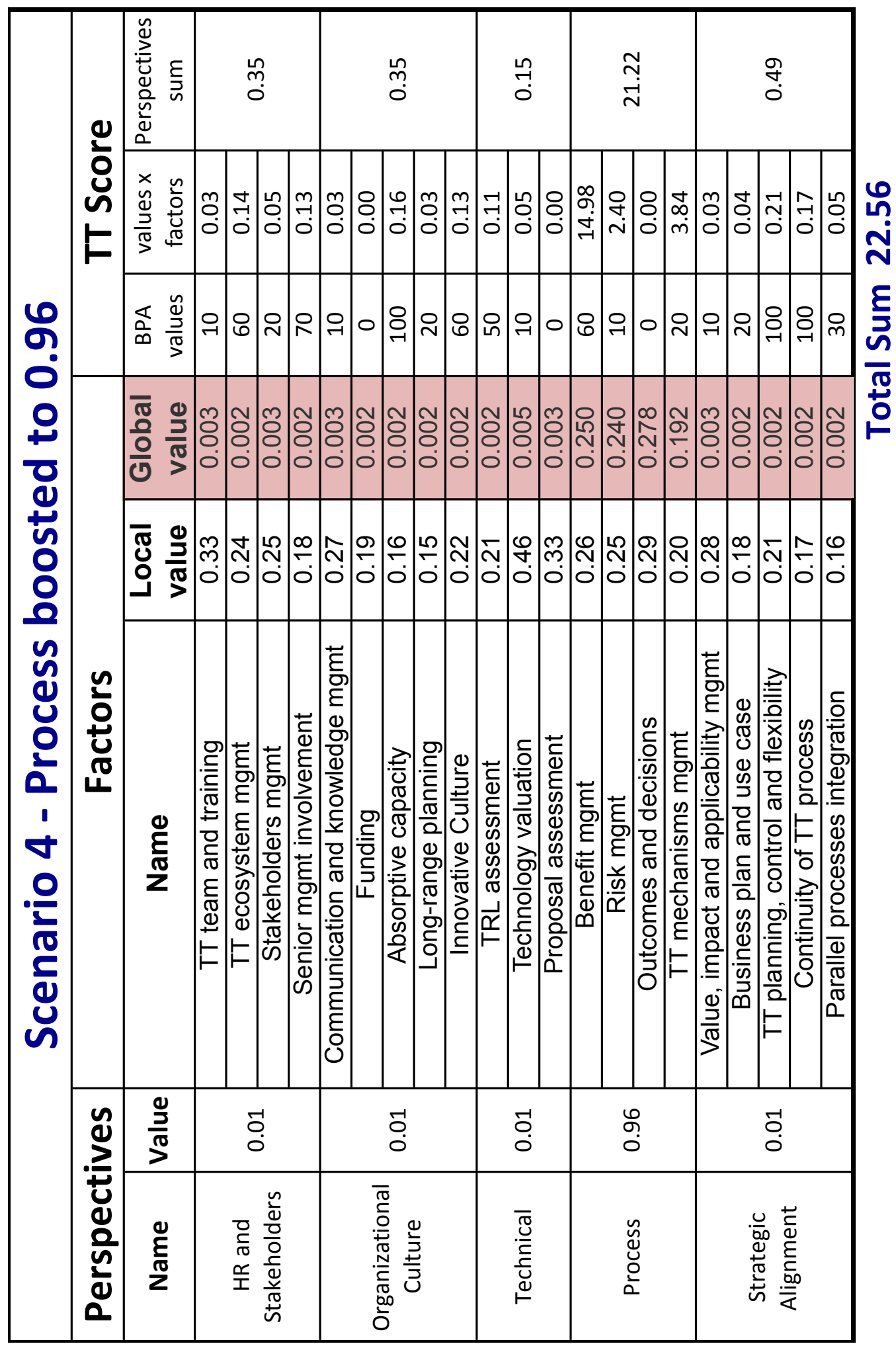


Table 63 - Sensitivity Analysis Scenario 5 Results

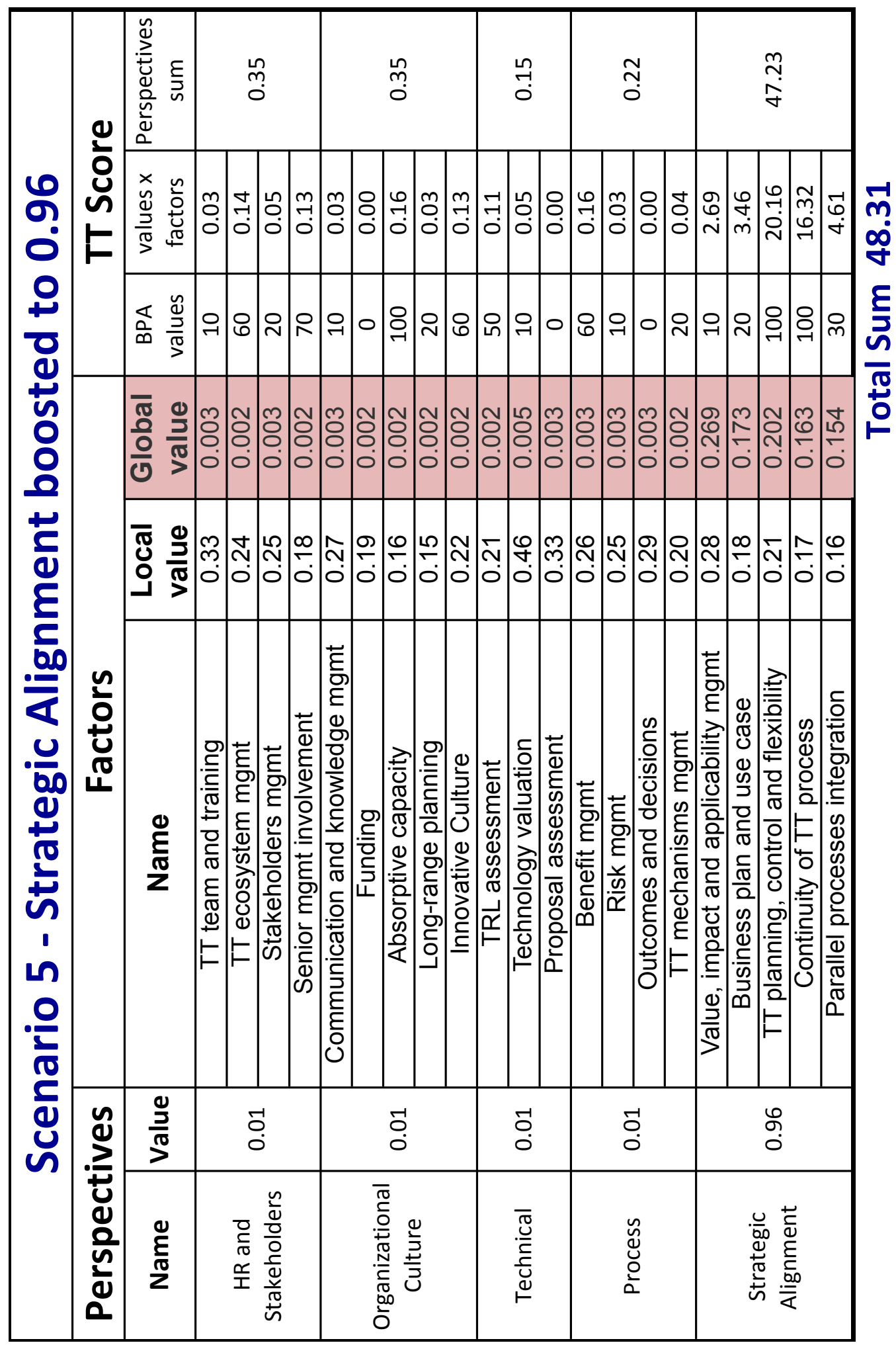


As expected, changing the perspectives relevance incurs in changes in the organizational TT score. In the case of BPA, the score hits its lowest level when the perspectives "Technical" or "Process" have their importance maximized (those are the worst-performing perspectives of the agency, according to the model application). Conversely, the score hits its highest level when the perspective "Strategic Alignment" has its importance maximized (that is the best-performing perspective of the agency, according to the model application). Table 64 brings a summary of the sensitivity analysis results.

Table 64 - Sensitivity Analysis Summary

\begin{tabular}{|c|c|c|}
\hline Scenario & Boosted Perspective & Org TT Score \\
\hline Original Application & None & 32.1 \\
\hline 1 & HR and Stakeholders & 35.1 \\
\hline 2 & Organizational Culture & 34.72 \\
\hline 3 & Technical & 15.91 \\
\hline 4 & Process & 22.56 \\
\hline 5 & Strategic Alignment & 48.31 \\
\hline
\end{tabular}

As previously indicated, these changes could inform managers on how to proceed with their efforts towards improving their technology transfer capabilities. 


\section{CHAPTER 6 - DISCUSSION}

This chapter brings a discussion of the model application results, both on a low level (within the boundaries of the specific case to which it was applied) and on a high level (what the application could mean to any organization). Additionally, a discussion on the generalizability of the research model is presented.

\section{Case Discussion}

\section{HR and Stakeholders}

This perspective contains factors related to the team, training, ecosystem, senior management, and other players involved.

The factor TT team and training is described in terms of the existence of a dedicated TT team, the background of the team members, its multidisciplinary nature, and the existence and frequency of TT training. The level attributed to the case organization was "dedicated TT team; not multidisciplinary; no training". The fact that there is a dedicated TT team in place is definitely a pro, and it reveals that the organization leaders have the right mindset concerning technology development. Nonetheless, as it was presented throughout the literature review section, TT is very complex and multidisciplinary, thus requiring an equally multidisciplinary team. Furthermore, the multidisciplinary nature of the TT team does not refer only to the background of those directly involved in the TT process, but also to the inclusion of representatives from various groups within the organization. This is not observed in the case organization (all members of the TT team come from the same group, 
responsible for managing research projects). Lastly, training is a very important practice that ensures consistency in organizational practices, as well as decreasing the hurdles of members leaving the team and new members coming in. The organization in question should strive to invite representatives from other departments to be part of the TT team, as well as devise a formal training strategy and cadence.

The factor "TT Ecosystem Management" is described in terms of relationships, cooperation, and partnerships with TT ecosystem members, e.g., TTO's, Science Parks, Research Centers, incubators, POC Centers, etc. The level attributed to the case organization was "Frequent interaction (at least monthly) with unstructured, ad-hoc discussions". The case organization has a positive history of creating relationships with ecosystem members, especially through funding third parties to develop technologies. Nonetheless, only a very limited number of those external relationships has evolved so much as to be a cooperation. Internal groups, conversely, have had the opportunity of becoming long-lasting partners, and to participate in successions of R\&D projects that would fit the classification of a program. Moreover, in the past the case organization has organized formal events, during which representatives from numerous organizations would gather and discuss future avenues for technology development in the sector, and the case organization would benefit by using those insights as to guide their technology development planning efforts. Recently however, these events have been scarcer and more limited in scope. To that point, a recommendation would be for the case organization to reinstate those events and to seek for formal R\&D collaboration agreements, in a way as to create R\&D programs, 
with the participation of multiple organizations aiming to achieve breakthrough innovations.

The factor "Stakeholders Management" is described in terms of interactions and cooperation with internal and external stakeholders to increase participation and create awareness within the boundaries of the TT process. The level attributed to the case organization was "TT team has frequent contact (at least monthly) with internal stakeholders (and external, if any); information goes one way (from the stakeholders, but with solicitation)". As already discussed, the fact that there is a formal TT team in place is a positive aspect of the case organization. Nevertheless, when it comes to the management of internal stakeholders, improvements could be made. The exchange of information does not seem to be as strong as one would desire, and additionally the TT team has to solicit crucial information by inquiring with project managers across the organization. The next factor, senior management involvement, could come into play when reinforcing the essentiality of collaboration and communication between TT stakeholders, making it easier for members of the TT team to have access to crucial information about research projects, and in a timely manner.

The factor "Senior Management Involvement" is described in terms of the awareness, approval, support, and active participation of senior managers in the TT process, including the indication of TT champions. The level attributed to the case organization was "Middle management is actively engaged in the TT process. Senior management is aware of the TT process, but their engagement is not consistent". As far as the involvement and support of the middle management, the case organization is very well served. Senior managers, on the other hand, could be more active in 
sponsoring and supporting the TT team and its activities. As aforementioned, a stronger involvement from senior managers and a clear support message would touch upon various pain points that hinder the TT team's work. A practical approach to improve their involvement is to appoint a TT champion (or rather a technology champion that would have several responsibilities, among which to support technology transfer and remove roadblocks that stand in the way of the TT team), which should be either a member of the senior management or someone with easy and constant access to the $\mathrm{C}$-suite.

\section{Organizational Culture}

This perspective contains factors related to organizational characteristics that should be in place for a better TT process.

The factor "Communication and Knowledge Management" is described in terms of the relevance and frequency of interactions between stakeholders, transfer and sharing of knowledge between different departments (outside the boundaries of the TT process). The level attributed to the case organization was "Interdepartmental communication is weak; only vital information is transferred from one department to another without solicitation". Reflecting what was seen in the factor "Stakeholders Management", the interdepartmental communication and collaboration in the case organization is not as strong as one would desire. Having in mind that knowledge sharing is essential to any successful technology transfer endeavor, this situation should be worrisome to managers. Efforts should be undertaken in order to increase the frequency and quality of information shared 
between groups, and to decrease the level of effort necessary for information to be shared.

The factor "Funding" is described in terms of ease of access and level of funding for transfer activities. The level attributed to the case organization was "no budget is allocated". This factor is probably the most straightforward in the entire model. Although the lack of a formal and structured budget for technology transfer activities does not necessarily mean that the organization is not using any resources to promote these activities, it would be recommended to allocate between $5 \%$ and $10 \%$ of the R\&D budget for TT.

The factor "Absorptive Capacity" is described in terms of capabilities related to identifying valuable information, assessing and using it. The level attributed to the case organization was "The organization has steady and successful partnerships, collaborative development or joint R\&D efforts and those amount to $25 \%$ to $50 \%$ of its research portfolio". As opposed to what was discussed in the factor "TT Ecosystem management", which dealt with the structured interaction, collaboration, and creation of R\&D programs with different entities within the technology transfer ecosystem, this factor discusses punctual collaborations with other entities. In this regard, the case organization has been performing very well. A significant share of their R\&D projects is conducted by external organizations, i.e. universities, research centers, and private companies, and this arrangement enables the case organization to have constant contact with different people, practices, and diverse skill sets that ultimately could bring benefits in terms of knowledge transfer. 
The factor "Long Range Planning" is described in terms of corporate strategy and long-term technology planning. The level attributed to the case organization was "formal corporate and business strategies exist but are tacitly propagated". Although the score attributed to the case organization for this factor is not the optimal one, efforts have been undertaken recently in order to improve it. Formal strategic guidelines do exist, and extensive efforts are being made aimed at promoting and reinforcing those guidelines in a more effective fashion.

The factor "Innovative Culture" is described in terms of "the organization ability and openness to implementing innovation and changes regarding its business practices". The level attributed to the case organization was "Minor innovations / changes in business practices have been successfully implemented in the last three years". As discussed earlier in this dissertation, the power sector is widely recognized as very stable and not prone to changes and innovation due to the very nature of the industry sector. Given this fact, one would expect the case organization to not have a high score in this factor. Nevertheless, with the impending major technological changes in the sector, the case organization has been keen to prepare for it (including with the creation of a business transformation office), and should be ready for the implementation of major business practices / models.

\section{Technical}

This perspective contains factors related to technical assessments and technology quantifications. 
The factor "TRL assessment" is described in terms of technology readiness levels and related metrics, e.g., IRL; SRL; RD3. The level attributed to the case organization was "technology readiness is assessed, and it informs the decisionmaking process regarding TT mechanisms and strategies". The case organization does have a formal TRL assessment for the research proposals considered for funding, but TRL is the only metric used. An improvement would be to make the process more consistent, and to implement further metrics as the ones described in the factor definition. Currently, research proponents are responsible for assessing the readiness, a process that could lead to biases and inconsistencies.

The factor 'Technology Valuation' is described in terms of technology assessment studies at several different points throughout the technology development cycle. The level attributed to the case organization was "one technology assessment study is conducted at the beginning of the technology development cycle". Although the frequency of assessments should be increased, efforts have been made to improve the evaluation process itself. Specifically, a project was conducted to create an evaluation framework that would fit the case organization's needs. The created framework is yet to be tested and later implemented.

The factor "Proposal Assessment" is described in terms of qualitative and quantitative assessments of research proposals. The level attributed to the case organization was "research proposals are not methodically assessed". Similarly to what happens in the previous factor "Technology Valuation", the assessment of proposals is not currently done in a methodical fashion, but an assessment model has 
been devised to tackle this gap. Pending its testing, the model should be implemented, thus improving the case organization performance in this factor.

\section{Process}

This perspective contains factors related to TT process features and characteristics.

The factor "Benefit Management" is described in terms of the identification, understanding, description, classification, and monitoring of benefits. The level attributed to the case organization was "benefits are differentiated from project deliverables, classified and quantified, without stakeholders' input or program considerations". The case organization has succeeded in detaching the strategic benefits of a research project from the mere achievement of a project's initial plans. However, it is still necessary to have a programmatic approach to benefits, as a way to link each individual project's outcomes to its topic technology roadmapping, and to inform future project selection, and technology planning.

The factor "Risk Management" is described in terms of the identification, understanding, description, classification, and monitoring of risks and opportunities. The level attributed to the case organization was "the TT risks and opportunities are identified". For the case organization, the positive point about its performance on this factor is that it is able to detach the technology transfer risks from the project management risks, i.e., even if the project is unfolding perfectly according to its plan, it does not necessarily mean that the outcome of that project will be easily transferred in to the technology recipient's operations. Nonetheless, the case organization has 
still to bring it to the next level, by quantifying the risks and devising different contingency plans for high impact / high probability risks.

The factor "Outcomes and Decisions" is described in terms of discussions, reports, and decisions made out of the TT process. The level attributed to the case organization was "non-standardized reports are produced; recommendations are made based on individual's "gut-feeling". This is certainly a white spot the case organization should be working on. Although the TT is well structured, with a team and a process in place, the project management perspective is still the main one considered in order to decide whether the project should be continued. Ideally, the technology transfer perspective should be contemplated and taken into account during stage-gates-like decision points at the same level as project management, and the case organization should strive to achieve that.

The factor "TT mechanisms management" is described in terms of the identification, understanding, description, classification, and monitoring of potential TT mechanisms. The level attributed to the case organization was "for each project, one TT mechanism is chosen ahead of time and a plan based on the mechanism is devised". As what can be seen in other factors within the process perspective, the case organization is not at the lowest level possible, but can certainly improve quite a bit. On a positive note, one TT mechanism is chosen and planned for ahead of time, but as discussed throughout this dissertation, technology development projects involve a considerable amount of uncertainty, which could lead to major changes, which in turn would require a flexible plan to accommodate those major changes. Having more than one technology transfer mechanism option is crucial when developing new 
technologies, and the case organization should be devoting some time and effort to implement a more thorough and flexible plan for each funded project.

\section{Strategic Alignment}

This perspective contains factors related to the alignment between $R \& D$ and business strategy, and the integration of TT with other managerial processes.

The factor "Value, Impact and Applicability Management" is described in terms of the economic value and strategic fit; level of impact of daily operations and beyond; Ease of applicability and extra requirements, such as adjustments, adaptations and special training. The level attributed to the case organization was "one assessment is made at the project start". Similar to what happens with the "Technology Valuation" factor, this factor is only dealt with through a one-time evaluation upfront, at the beginning stages of the project. As explained earlier, technology development projects are very dynamic and uncertain endeavors, and require multiple and thorough assessment points. The framework created for technology valuation focuses on the financial return of any given project. In order to suit the needs of this factor, another framework could be created in order to measure the strategic fit of $R \& D$ projects, and as the factor levels definitions suggest, assessments should be conducted in a dynamic fashion, as to respond to changes regarding the project, technology, market, and corporate strategy.

The factor "Business Plan and Use Case" is described in terms of planning of the technology application, based on benefits, value, impact, and applicability. The level attributed to the case organization was "use cases are available and it has most 
of the required information". In spite of the fact that the case organization does write use cases that contain most of the relevant information, the leap between this level and the next one is quite considerable (from 20 points to 100 points). The case organization should identify the missing information, and strive to capture it as thoroughly and as early as possible in order to inform the project selection process.

The factor "TT Planning, Control, and Flexibility" is described in terms of the continuous improvement of the TT process, such as meetings, planning, reviews, setting objectives and priorities, and adjusting those based on market and strategy changes. The level attributed to the case organization was "annual meetings are conducted to review the process against strategic management guidelines; set priorities and objectives; identify and reflect on lessons-learned". In this factor the case organization is performing perfectly. Conducting no process review meetings is obviously not a best practice. However, in seeking to continuously improve any given process, an organization might as well devote too much time and attention to it, reaching what is called the "analysis paralysis" status.

The factor "Continuity of TT Process" is described in terms of length and comprehensiveness of TT process. The level attributed to the case organization was "TT process starts in the proposals and ideas assessment phase even before the technology development project starts. This is another good example for the case organization. Rather than approaching technology transfer only after everything was said and done in the technology development cycle, the case organization strives to tackle TT issues from the time a research proposal is submitted. 
The factor "Parallel Processes Integration" is described in terms of the integration, feedback, and information exchange from and to other managerial processes, e.g., project, portfolio and program management, and roadmapping. The level attributed to the case organization was "TT process exchanges information with project management; stage-gates decisions take the TT perspective into account". As discussed in the "TT Decisions and Outcomes" factor, the TT perspective is taken into account, but it is not as strong as project management aspects when deciding over a particular research project. In addition, technology transfer does not seem to be seamlessly integrated with the other technology management processes. An effort should be made to change that situation, especially in the interactions between TT and technology roadmapping, in order to improve the overall technology management capabilities of the case organization.

\section{Improvement Simulation}

In light of the results presented in the Chapter 4 (Research Model Development), and the discussion conducted in this chapter about each individual factor, it is informative to simulate what the final TT score would look like for BPA once the agency is able to reach excellence, which is to reach 100 points as described by the desirability curves in the five most important factors in the model, namely "Technology Valuation", "TT Team and Training", "Value, Impact and Applicability", "Stakeholders Management", and "Proposal Assessment". Table 65 presents the simulated score: 
Table 65 - Simulation of Top Five Factors Improvement

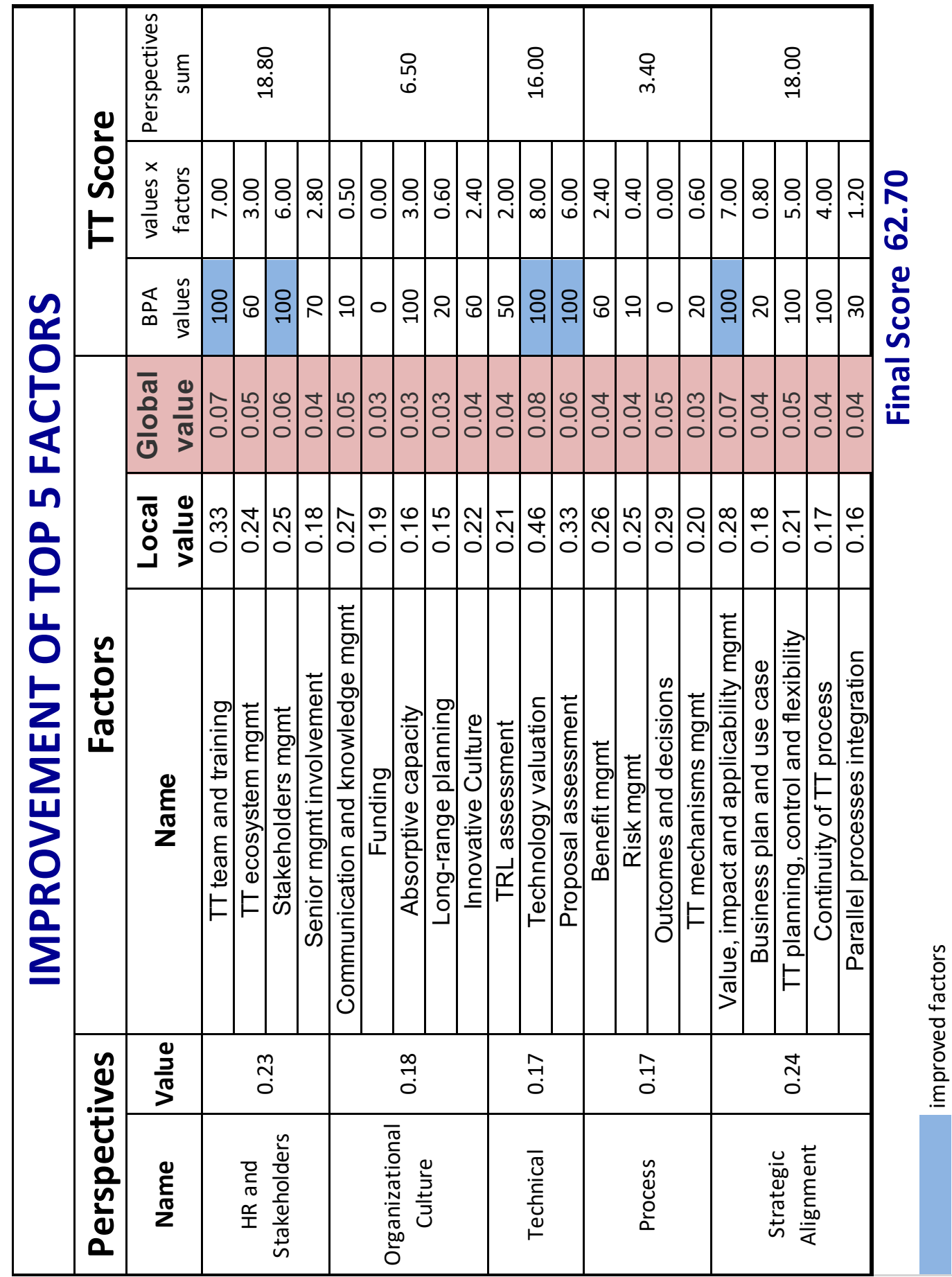


As evidenced by the simulation, working on reaching excellence in the top five factors in the model can lead to an extraordinary increase in the overall technology transfer score. In this case, BPA would have its score increased by more than 95\%, from 32.10 up to 62.70 .

It is also interesting to note that even when achieving the maximum score for the five top factors in the model, BPA would still be far from the overall maximum of 100 points. That situation sheds a light onto two very important aspects of the model and its application. Firstly, it is not sufficient to focus solely on the most important factors in order to achieve excellence in the whole process, although adopting that strategy would definitely maximize the improvement of the score. Secondly, and perhaps most importantly, the model can be seen as a maturity model, in a way as to depict the maximum level of performance for each factor as a perfect situation, which in reality may not be attainable for all twenty-one factors at the same time. Interpreting the model in such a way, the technology manager would prefer to start prioritizing efforts in order to improve the organization's performance, and at the same time understanding that achieving 100 points might not be feasible, or if it is feasible, it would not be worthwhile due to the level of resources one would have to spend in order to get there. All in all, even when regarding the maximum level of performance in the model as an ideal but not achievable scenario, it would still be informative and beneficial to organizations as a way as to guide them and "force" them to always strive towards that unattainable goal. 


\section{General Discussion}

Having discussed the results of the specific case to which the model was applied, it is important to zoom out and discuss the implications such a model could have in the technology transfer capabilities of an organization, and also the strategies organizations could adopt to make improvements to its processes.

Discussions were held with practitioners, where technology managers were asked about the usefulness of the model and the potential it would have in helping them in their daily activities. Out of the discussion, three main ways in which the model would be beneficial were identified: The model itself, the application process, the results and its analysis.

The model itself is seen as a benefit once it portrays an ideal technology transfer. By looking at the model, and its structure with its components and its definitions, a technology manager would be able to grasp what an ideal technology transfer process should look like. That being said, practitioners should be aware that the specifics of each organization's reality will dictate the extent to which that ideal process is attainable, i.e. not all factors would be able to be present (and at the required level of excellence) simultaneously in all organizations. Nonetheless, each technology manager should be able to identify which factors are unattainable and adapt the model accordingly. All in all, practitioners agree that, once understood, the model itself brings benefits of its own.

The application process is seen as a benefit once it would force practitioners to scrutinize their organizations and their processes. This scrutiny, coupled with the discussions generated within and across different departments, would ultimately 
lead to a better understanding of the organization, its weaknesses, and its strengths. The situational awareness brought about by the model application enables managers to emphasize areas of deficiencies in order to generate efforts towards achieving technology transfer excellence.

The results and its analysis are seen as a benefit once it enables the measurement of successes and/or failures overtime. When an organization / department applies the model and analyzes its results multiple times, it can compare and contrast its technology transfer process status quo against itself in the past, as well as against other organizations / departments. Moreover, practitioners agree that going through these application/results analysis cycles would set a good example for other parts of the organization on how to continuously improve, how to be welcoming to changes in processes and business models, and thereby always aiming to learn from its own mistakes and successes.

Furthermore, by looking at the results a manager could choose different paths to tackle the identified issues, especially when taking into consideration the importance of perspectives within which factors are grouped. For instance, all factors under the perspective "Process" might have received poor scores, prompting the organization to focus on ways to improve those factors. Nonetheless, if the "Process" factors are performing poorly, but the "Process" perspective is the lowest in importance, perhaps it would be more advantageous for the organization to focus first on improving other perspectives, thereby always striving to increase the overall TT score as much as possible. Adopting such a strategy would leverage the holistic nature of the decision model, i.e., having applied a model that takes into consideration 
aspects that describe the technology transfer process in a thorough fashion, the manager sees himself / herself in a position to prioritize efforts that will lead to the maximization of process improvement with each initiative taken. While the aforementioned strategy could be the most logical to many technology managers, it is not the sole one. Another strategy that could be recommended is to take care of the "low hanging fruits" first. Each organization should know itself well enough to make the judgment call and to identify the "low hanging fruits", i.e. the easiest improvement points. Once those are identified, they should be dealt with, even if they are not the most important according to the model overall importance values. The idea is that since those are the easiest to deal with, the organization would tackle them more quickly and then learn during the process, making it easier for a second stage, when they would try to deal with more difficult, effort-demanding factors. Such a strategy acknowledges that there is always a learning curve when implementing process improvements, and it would be easier and safer to deal with less complex issues while everyone involved is trying to wrap their heads around the changes. Later on, when people are more used to and confident with the changes, the organization would face less hurdles and barriers when dealing with more complex issues.

Notwithstanding the fact that the model was applied to an organization in the power sector, and that several subject matter experts used in the validation and quantification processes were also related to the energy realm in one way or another, the model itself was never intended to be tailored to energy-related applications. When looking at the components of the model, i.e. its perspectives and factors, it is easy to perceive that nothing applies exclusively to the energy sector. In reality, the 
model components represent important aspects of technology transfer, irrespective of the application sector, and that can be attested by the significant breadth of expertise in the expert panels, most notably from the representatives of academia. That aspect, combined with the extensive literature review conducted prior to the model development, leads to the conclusion that this research model is applicable to virtually any organization that deals with technology transfer issues.

Having said that, one caveat must be mentioned. A prudent technology manager would, prior to the model application, assess to what extent the importance of each perspective and factor in the model reflects the reality of the organization undertaking the evaluation. Given the fact that cultural, economic, and political environments, as well as competitive environment, organizational structure, and product-related aspects could vary significantly from one organization to another (or from one region or country to another, for that matter), it might be best, and depending on circumstances, for the technology manager to redo the model quantification prior to its application. This would assure that the importance of each perspective and each factor adheres to the reality and needs of the specific organization using the model. 


\section{CHAPTER 7 - CONCLUSION}

This chapter brings a recapitulation of the study presented in this dissertation, with the steps taken to complete it. It also brings a discussion on how this study contributes to the body of knowledge, both from a practical and a theoretical standpoint. Finally, limitations are discussed, and future research opportunities are identified.

The first step of this study was to conduct a literature review on technology transfer and related topics, which resulted in the identification of research gaps. A new methodological approach (hierarchical decision modeling aided by action research) was developed and employed to create the research model. The next step was to apply the model to the Bonneville Power Administration, a large federal agency responsible for marketing electricity in the Pacific Northwest region of the United States. Following the model application, the results were analyzed, and insights were generated, both in terms of the specific case to which the model was applied, as well as in terms of its usefulness to technology managers in general.

While acknowledging the vast amount of research that has been conducted on the technology transfer topic in the last forty years, the problem statement was framed in terms of the need organizations have to improve their technology transfer capabilities, which would lead to more effective and efficient research and development efforts, which in turn would lead to competitive advantage in the globalized and innovation-driven economic arena of the $21^{\text {st }}$ century. The research objective was to create a scoring model to help organizations assess their current technology transfer capabilities, and to inform them on how to proceed about making 
improvements. This research has achieved such objective, as it will become clear when the individual research gaps and research questions are discussed.

\section{Research Contributions}

From a practitioner's standpoint, this research is able to help in at least four distinct ways: The identification of perspectives and factors that are not only vital for achieving excellence in technology transfer, but also depict the process in its entirety, unlike what is observed across existing literature; the model serves as a tool to measure current technology transfer capabilities of any organization; the results of the model application bring situational awareness and insights on how to improve the current process; and the model and its application bring specific insights on how to improve the strategic alignment between research and development activities and overall business strategy.

The model developed and applied in this study is, first and foremost, a situational awareness tool. Technology transfer process improvements are not achieved directly through the model application but are rather enabled by it. That is not to diminish the importance of the model in any way, shape, or form. As it is commonly said "one cannot improve what one cannot measure". The model serves as the measuring tool for the technology manager. After its application and brought about by the desirability curves, the manager should be able to identify the strengths and weaknesses of the organization in light of best practices.. Furthermore, the model application results would serve to inform the manager on how to organize his/her improvement initiatives. The model itself could be seen as a north star, a direction to 
be followed at all times. Moreover, the history of multiple sequential TT scores assessed throughout time could serve as evidence and measure of progress, i.e. after understanding the weaknesses, designing and implementing action plans, the technology manager should repeat the model application to check the delta between the current status and the previous ones, and new insights and strategic discussions can be generated by analyzing these deltas. Lastly, ancillary benefits could be expected from the application of this model by striving to achieve excellence in every perspective and factor. The organization will inevitably increase its knowledgesharing and communication abilities within the technology-related departments and across different departments, and the alignment between technology development projects and business strategy is increased through the observation of the directions contained in the "Strategic Alignment" perspective, with its factors and desirability curves.

From a theoretical standpoint, there are two main contributions of this research: The extension of hierarchical decision modeling usage by ways of a methodological novelty brought about by action research, and new factors being included into the technology transfer body of knowledge.

The methodological novelty was characterized by mixing literature review with action research in the process of building the HDM model. As explained earlier in this dissertation, the standard process of creating an HDM model consists of identifying important criteria for the topic/problem in question, relying solely on past research and projects. The assumption is that the researcher, who is not a technical expert, cannot include any criterion in the model if there is not heavy support for that 
criterion demonstrated in the literature. Later on, during the model validation process, subject matter experts will judge if each criterion should remain in the model, and will also be able to add any important criteria not currently considered.

The present research adopted a different approach, in which the researcher conducted an action research project concurrently with the literature review and used criteria from both sources in order to build the initial model. In the implementation of this novel approach, the model validation process would be important in checking the validity and feasibility of such an approach. If the subject matter experts were to reject the majority of criteria brought about by the action research project, the methodological novelty would be invalidated. Nonetheless, as exposed in the research model development chapter, only one of the criteria brought about by the action research was rejected and dropped from the model. This positive result has validated not only the model itself, but also this new approach in building an HDM model, one that uses action research as a way to incorporate practitioners' real-life challenges and experience early on in the model development process. Additionally, results from the model quantification process help to further validate the methodological novelty, and prove that the approach is not only valid, but was instrumental in building a strong and robust model. Figure 45 shows the global importance of the twenty-one factors in the model, taken all together, without considering the perspectives to which each one belongs, and highlighting the top five and bottom five. 


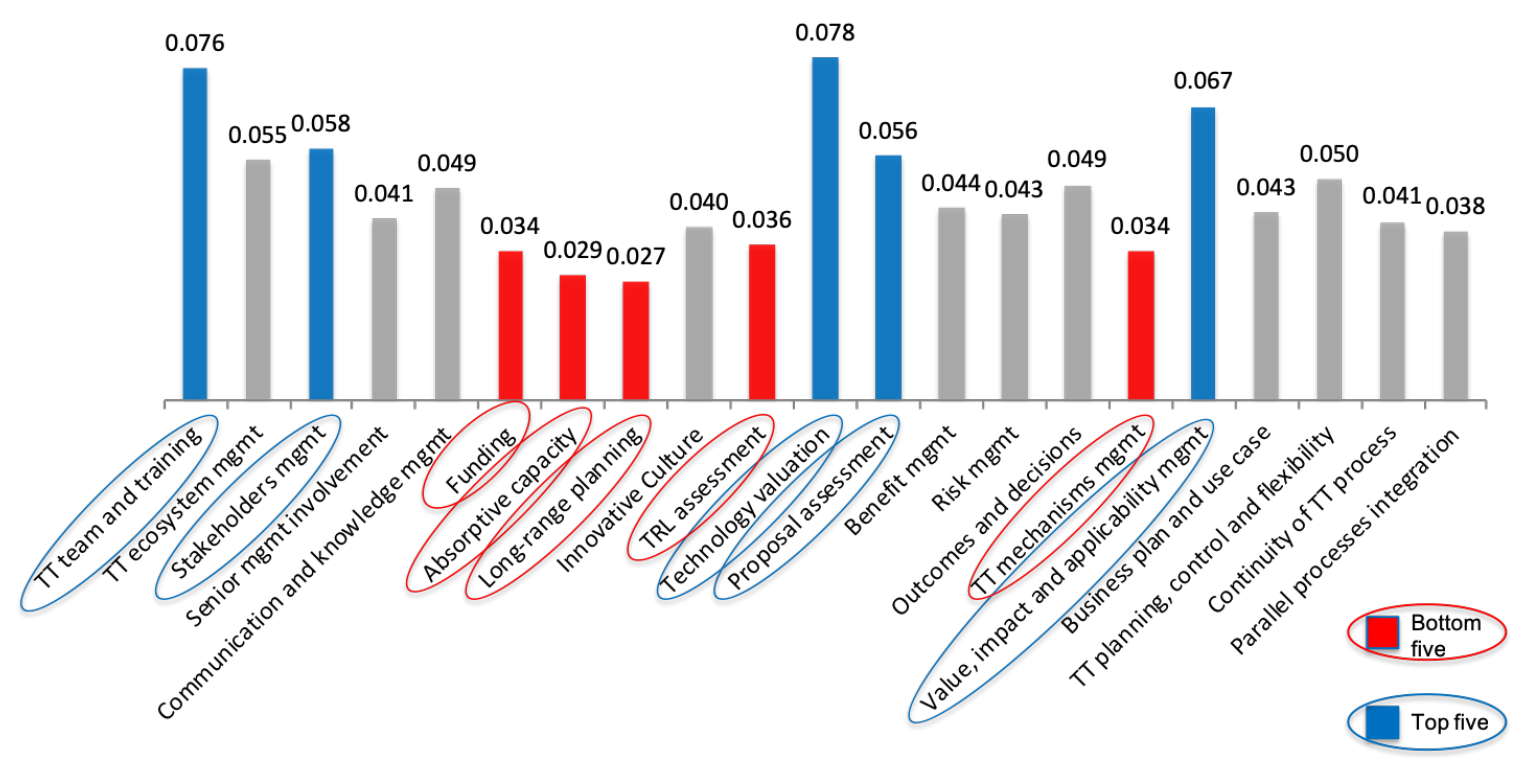

Figure 45 - Top five and Bottom Five Factors

As shown in Figure 45, from the top five factors in the model, two came from the action research project (Stakeholders Management; and Value, Impact, and Applicability Management). In other words, using the action research piece to complement literature review in building the HDM model was paramount to the success of this research. Furthermore, when breaking down the model and showing the factors importance grouping them by perspective, the pattern is the same. As shown in Figure 46, two of the top five factors per perspective came from the action research piece "Outcomes and Decisions", which was the top factor in the "Process" perspective, and "Value, Impact, and Applicability Management", which was the top factor in the "Strategic Alignment" perspective. 


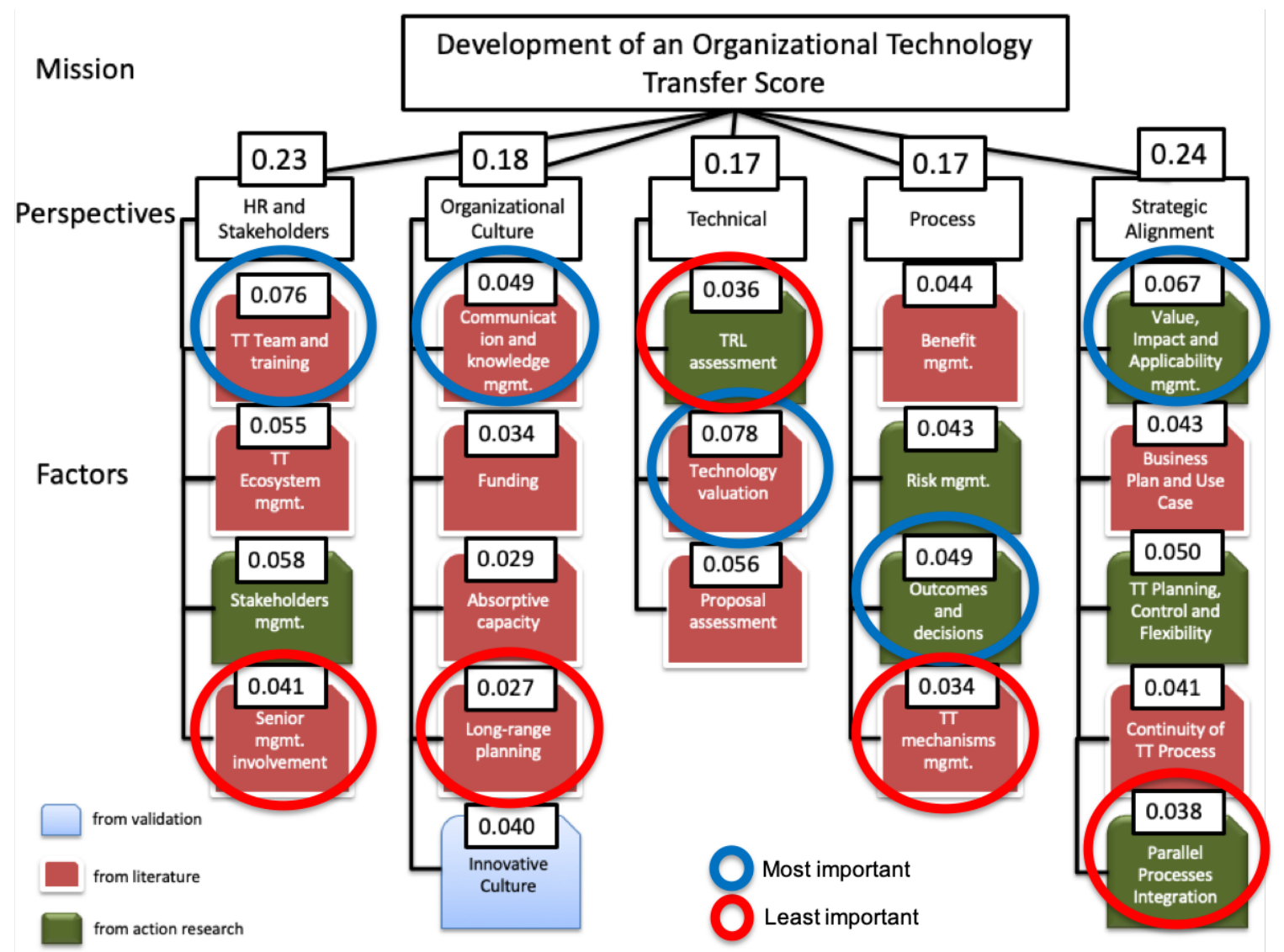

Figure 46 - Most and Least Important Factors per Perspective

The second main theoretical contribution spawned directly from the first one:

The criteria identified during the action research that were added to the model and later validated by the subject matter experts were not initially part of the technology transfer body of knowledge. Now, however, those criteria can be considered integral part of the technology transfer literature, possibly giving rise to new research to further use and investigate them.

As a secondary theoretical contribution, one could mention the fact that the quantification of the model perspectives further highlighted the importance of intensifying the research on the strategic alignment topic. It is interesting to note that, 
in spite of being the least researched aspect of technology transfer, "Strategic Alignment" was ranked as the most important perspective leading to the calculation of the organizational TT score. Such a result serves as evidence of the need for more research on how to align R\&D activities and corporate strategy, corroborating what was discussed in the fourth research gap identified in this study.

There were four research gaps identified and tackled by this research. The first one and the third one are, respectively: "there are not enough holistic and quantitative models to assess and measure an organization's TT capabilities" and "there is a lack of studies on internal TT overall". The research model itself tackled both of these gaps, by bringing a comprehensive depiction of the factors that should be in place in order to build a proper technology transfer process, and also focusing on internal technology transfer activities. The second research gap, "there is a lack of studies on how to improve an organization's TT capabilities based upon its current status" was tackled by the desirability curves and the discussion on different strategies to achieve improvement. The curves and its levels present the technology manager with a path that should be followed in order to improve at each and every factor. Moreover, the improvement strategies presented give the manager a variety of ways he/she could choose from and implement depending on each individual organization's characteristics. The fourth and last research gap, "There is a lack of studies on TT helping to align R\&D and business strategy", was tackled by both the strategic alignment perspective within the model, and the literature review and discussion on technology transfer as a research and development alignment tool. Similarly to what happens to the rest of the model, the strategic alignment perspective informs the 
manager on the factors that should be in place to improve the strategic adherence of $\mathrm{R} \& \mathrm{D}$, and the literature review and discussion serves both practitioners and scholars on how to understand and further explore the topic.

There were also five research questions posed at the beginning of the dissertation. The first one, "What are the most and least important criteria and factors when assessing TT capabilities?", is responded to by the model components as a whole. The second question, "What are the practices and factors that influence the $R \& D$ alignment with business strategy?" are responded to by the specific factors contained in the strategic alignment perspective. The third question, "How should an organization proceed about its TT weaknesses after undertaking an assessment?", is responded to by both the desirability curves and its levels, and the discussion on the various strategies to achieve improvements. The fourth question, "What are the most and least important criteria and factors when assessing TT capabilities?", is responded to by the results of the model quantification phase. Lastly, the fifth question, "Is the model generalizable to other sectors and applications?", is responded to by the fact that none of the model components speak only to one industry sector in particular. This was complemented by the discussions that were had with all participating subject matter experts, who showed no concern whatsoever regarding the applicability of the model to other cases. A more in-depth discussion on the generalizability topic is presented later on in this chapter. 


\section{Limitations and Future Research Opportunities}

This research has its limitations, and those are acknowledged herein. The first limitation to be mentioned is that of the method itself. Hierarchical decision modeling, in spite of its great attributes and strengths, is based on subjective judgments from subject matter experts, who in turn are human beings and therefore inherently biased to some extent. Notwithstanding this fact, there are techniques available to minimize this bias. Most importantly during the subject matter experts identification and expert panels formation phases, techniques such as social network analysis, snowball sampling, and the allocation of expert panels based on academic/professional background and past experience, can be used. This research has used these techniques, to ensure that expert biases were under control and not impairing an objective interpretation of the results.

The second limitation to mention is that of the model generalizability. The model was built to focus on internal technology transfer issues and to be applied to an organization from the power industry in the Pacific Northwest region of the United States. For this application in particular, the model has been thoroughly validated, as well as its results. However, because of the fact that the model has not been applied to multiple scenarios, when it comes to other types of application, such as different industrial sectors, or even different types of technology transfer, e.g. external, international, competitive, the model might or might not need to have its factors and perspectives validation and quantification phases redone. While prudence dictates that technology managers should be aware of this limitation and use their best judgment and discretion to evaluate the extent to which it could impact the model 
application, it is important to notice that none of the model components, be it perspectives, factors, or desirability curves, are related to only one industrial sector, organization type, or technology transfer type. In fact, in discussions with the participating experts, there was a consensus around the idea that the model application could be easily and readily replicated to virtually any sector or technology transfer type. Additionally, the pool of experts used in this study was composed of a very heterogeneous and diverse set of individuals, representing different types of educational backgrounds, as well as different industries and different activity sectors, i.e. academia, industry, and government. A follow-up research opportunity is to step back and remake the quantification process, only this time using various sets of expert panels, each one representing one sector in specific. In doing so, the researcher will likely to be able to analyze the differences between these sectors / industries in the way they regard technology transfer, if any.

The third limitation to mention is with regards to the scope of the study. The application conducted in this study was that of assessing the technology transfer capabilities of one single organization, aimed at providing that organization with the knowledge and tools to improve its capabilities. While the study has achieved its objective, the model itself can be used with an additional purpose: That of comparing technology transfer capabilities of different organizations, or of different groups within the same organization. Future researchers could use the model to compare the technology transfer process performance across different industries or organizations, as well as to rank different companies based on their technology 
transfer capabilities, aiming to help organizations to choose the best partnership for a collaborative R\&D project, for instance.

Lastly, another future research opportunity is to investigate the feasibility of an endeavor to create a high-level managerial framework to integrate the several processes and tools that compose the research and development realm, e.g., roadmapping, portfolio management, project management, technology transfer, among others. The aim should be to replicate the assessment capabilities of this model, but to expand it beyond technology transfer, as to integrate every major managerial process that comes into play when managing technologies. 


\section{REFERENCES}

[1] D. Nobelius, "Linking product development to applied research: transfer experiences from an automotive company," Technovation, vol. 24, no. 4, pp. 321-334, Apr. 2004.

[2] S. N. Bar-Zakay, "Technology transfer model," Technol. Forecast. Soc. Change, vol. 2, no. 3-4, pp. 321-337, 1971.

[3] B. Bozeman, "Technology transfer and public policy: a review of research and theory," Res. Policy, vol. 29, no. 4-5, pp. 627-655, 2000.

[4] A. Talaei, M. S. Ahadi, and S. Maghsoudy, "Climate friendly technology transfer in the energy sector: A case study of Iran," Energy Policy, vol. 64, pp. 349-363, 2014.

[5] E. Worrell, R. van Berkel, Z. Fengqi, C. Menke, R. Schaeffer, and R. O. Williams, "Technology transfer of energy efficient technologies in industry: a review of trends and policy issues," Energy Policy, vol. 29, no. 1, pp. 29-43, 2001.

[6] J. Estep, "Development of a technology transfer score to inform the selection of a research proposal," Portland Int. Conf. Manag. Eng. Technol., vol. 2015-Septe, pp. 1754-1768, 2015.

[7] B. Bozeman, H. Rimes, and J. Youtie, "The evolving state-of-the-art in technology transfer research: Revisiting the contingent effectiveness model," Res. Policy, vol. 44, no. 1, pp. 34-49, Feb. 2015.

[8] M. Robbins and J. Milliken, "Technology-Transfer and Process of Technological Innovation - New Concepts, New Models," R Manag., vol. 6, pp. 165-170, 1976.

[9] Thomas Magnusson and Glenn Johansson, "Managing internal technology transfer in complex product development," Eur. J. Innov. Manag., vol. 11, no. 3, pp. 349-365, Aug. 2008.

[10] J. Morgan, "Five Examples of Companies With Internal Innovation Programs," Huffington Post, 09-Apr-2015. .

[11] J. Owyang, "The Ten Types Of Corporate Innovation Programs," Huffington Post, 04-Aug-2016. .

[12] D. Schawbel, "Jeremiah Owyang: Why Companies Need An Innovation Program," Forbes, 24-Feb-2017. [Online]. Available: http://www.forbes.com/sites/danschawbel/2017/02/24/jeremiah-owyangwhy-companies-need-an-innovation-program/. [Accessed: 24-Apr-2017].

[13] A. Kadareja, "Internal and Hidden Risks of Innovation Projects," Innovation Management, 15-Jul-2013. [Online]. Available: http://www.innovationmanagement.se/2013/07/15/internal-and-hiddenrisks-of-innovation-projects/. [Accessed: 24-Apr-2017].

[14] S. Curtis, "Four in five technologies fail to cross the 'Valley of Death,'” Techworld, 02-May-2013. [Online]. Available: http://www.techworld.com/news/apps/four-in-five-technologies-fail-crossvalley-of-death-3445285/. [Accessed: 21-Apr-2017].

[15] B. Baruah and A. Ward, "Metamorphosis of intrapreneurship as an effective organizational strategy," Int. Entrep. Manag. J., vol. 11, no. 4, pp. 811-822, 2015. 
[16] B. Altringer, "A New Model for Innovation in Big Companies," Harvard Business Review, 19-Nov-2013. [Online]. Available: https://hbr.org/2013/11/a-newmodel-for-innovation-in-big-companies. [Accessed: 24-Apr-2017].

[17] M. Zwilling, "10 Ways For Startups To Survive The Valley Of Death," Forbes, 18Feb-2013. [Online]. Available:

http://www.forbes.com/sites/martinzwilling/2013/02/18/10-ways-forstartups-to-survive-the-valley-of-death/. [Accessed: 21-Apr-2017].

[18] M. Rogowski, “Tesla Racing Out Of The 'Valley Of Death' Onto The Road Ahead," 08-May-2013. [Online]. Available:

https://www.forbes.com/sites/markrogowsky/2013/05/08/tesla-racingout-of-the-valley-of-death-onto-the-road-ahead/\#194192fd56a0. [Accessed: 21-Apr-2017].

[19] J. Estep, "Development of a Technology Transfer Score for Evaluating Research Proposals: Case Study of Demand Response Technologies in the Pacific Northwest," Diss. Theses, Feb. 2017.

[20] E. G. Carayannis, I. N. Dubina, and A. A. Ilinova, "Licensing in the Context of Entrepreneurial University Activity: an Empirical Evidence and a Theoretical Model," J. Knowl. Econ., vol. 6, no. 1, pp. 1-12, Mar. 2015.

[21] L. Zhao and A. Reisman, "Toward meta research on technology transfer," IEEE Trans. Eng. Manag., vol. 39, no. 1, pp. 13-21, 1992.

[22] J. Heinzl, A.-L. Kor, G. Orange, and H. R. Kaufmann, "Technology transfer model for Austrian higher education institutions," J. Technol. Transf., vol. 38, no. 5, pp. 607-640, Oct. 2013.

[23] 0. Meseri and S. Maital, "A survey analysis of university-technology transfer in Israel: evaluation of projects and determinants of success," J. Technol. Transf., vol. 26, no. 1, pp. 115-125, 2001.

[24] E. M. Rogers, S. Takegami, and J. Yin, "Lessons learned about technology transfer," Technovation, vol. 21, no. 4, pp. 253-261, 2001.

[25] W. Liu, "A Quantitative Technology-Transfer Model and Its Application to Aircraft Engines," Technol. Forecast. Soc. Change, vol. 44, no. 2, pp. 179-186, Sep. 1993.

[26] F. Amesse and P. Cohendet, "Technology transfer revisited from the perspective of the knowledge-based economy," Res. Policy, vol. 30, no. 9, pp. 1459-1478, 2001.

[27] E. G. Carayannis, "Knowledge transfer through technological hyperlearning in five industries," Technovation, vol. 19, no. 3, pp. 141-161, Jan. 1999.

[28] E. M. Rogers, Diffusion of Innovations. 1983.

[29] D. F. Halpern, Thought and knowledge: An introduction to critical thinking (2nd.). Hill sdale. NJ: Lawrence. Erlbaum A ssociates. 連結, 1989.

[30] E. G. Carayannis and D. F. J. Campbell, “Open Innovation Diplomacy and a 21st Century Fractal Research, Education and Innovation (FREIE) Ecosystem: Building on the Quadruple and Quintuple Helix Innovation Concepts and the 'Mode 3' Knowledge Production System," J. Knowl. Econ., vol. 2, no. 3, p. 327, Sep. 2011. 
[31] E. G. Carayannis, D. Popescu, C. Sipp, and M. Stewart, “Technological learning for entrepreneurial development (TL4ED) in the knowledge economy (KE): Case studies and lessons learned," Technovation, vol. 26, no. 4, pp. 419-443, Apr. 2006.

[32] E. G. Carayannis, E. M. Rogers, K. Kurihara, and M. M. Allbritton, "Hightechnology spin-offs from government R\&D laboratories and research universities," Technovation, vol. 18, no. 1, pp. 1-11, Jan. 1998.

[33] E. G. Carayannis, A. Y. Cherepovitsyn, and A. A. Ilinova, “Technology commercialization in entrepreneurial universities: the US and Russian experience," J. Technol. Transf., vol. 41, no. 5, pp. 1135-1147, Oct. 2016.

[34] S. Barzakay, "Technology-Transfer from Defence to Civilian Sector in Israel Methodology and Findings," Technol. Forecast. Soc. Change, vol. 10, no. 2, pp. 143-158, 1977.

[35] R. Seaton and M. Cordeyhayes, "The Development and Application of Interactive Models of Industrial-Technology Transfer," Technovation, vol. 13, no. 1, pp. 45-53, Jan. 1993.

[36] D.-H. Baek, W. Sul, K.-P. Hong, and H. Kim, "A technology valuation model to support technology transfer negotiations," R Manag., vol. 37, no. 2, pp. 123138, Mar. 2007.

[37] D. Leonardbarton and D. Sinha, "Developer-User Interaction and User Satisfaction in Internal Technology-Transfer," Acad. Manage. J., vol. 36, no. 5, pp. 1125-1139, Oct. 1993.

[38] K. Malik, "Aiding the technology manager: a conceptual model for intra-firm technology transfer," Technovation, vol. 22, no. 7, pp. 427-436, Jul. 2002.

[39] L. R. Mejia, "A brief look at a market-driven approach to university technology transfer: One model for a rapidly changing global economy," Technol. Forecast. Soc. Change, vol. 57, no. 3, pp. 233-235, Mar. 1998.

[40] R. Landry, N. Amara, J.-S. Cloutier, and N. Halilem, "Technology transfer organizations: Services and business models," Technovation, vol. 33, no. 12, pp. 431-449, Dec. 2013.

[41] M. Bommer, R. Janaro, and D. Luper, "A Manufacturing Strategy Model for International Technology-Transfer," Technol. Forecast. Soc. Change, vol. 39, no. 4, pp. 377-390, Jul. 1991.

[42] G. Festel, "Similarities of Successful Technology Transfer Through New Ventures," Int. J. Innov. Manag., vol. 19, no. 2, p. UNSP 1550025, Apr. 2015.

[43] J. Zhao, J. Zhou, and Y. Tong, Knowledge-integration-based Technology Transfer Mode for Manufacturing Firms. New York: Ieee, 2008.

[44] G. Festel, "Academic spin-offs, corporate spin-outs and company internal startups as technology transfer approach," J. Technol. Transf., vol. 38, no. 4, pp. 454-470, Aug. 2013.

[45] D. Prud'homme, M. von Zedtwitz, J. J. Thraen, and M. Bader, "Forced technology transfer' policies: Workings in China and strategic implications," Technol. Forecast. Soc. Change, vol. 134, pp. 150-168, 2018. 
[46] L. Wan, B. Luo, T. Li, S. Wang, and L. Liang, "Effects of technological innovation on eco-efficiency of industrial enterprises in China," Nankai Bus. Rev. Int., vol. 6, no. 3, pp. 226-239, 2015.

[47] R. Belderbos, V. Van Roy, and F. Duvivier, "International and domestic technology transfers and productivity growth: firm level evidence," Ind. Corp. Change, vol. 22, no. 1, pp. 1-32, 2012.

[48] N. Reddy and L. Zhao, "International Technology-Transfer - a Review," Res. Policy, vol. 19, no. 4, pp. 285-307, Aug. 1990.

[49] A. C. Seror, "Action research for international information technology transfer: A methodology and a network model," Technovation, vol. 16, no. 8, pp. 421429, Aug. 1996.

[50] P. Chang, C. Shih, and C. Hsu, "The Formation Process of Taiwan Ic Industry Method of Technology-Transfer," Technovation, vol. 14, no. 3, pp. 161-171, Apr. 1994.

[51] T. Waroonkun and R. A. Stewart, "Modeling the international technology transfer process in construction projects: evidence from Thailand," J. Technol. Transf., vol. 33, no. 6, pp. 667-687, Dec. 2008.

[52] A. de la Tour, M. Glachant, and Y. Ménière, "Innovation and international technology transfer: The case of the Chinese photovoltaic industry," Energy Policy, vol. 39, no. 2, pp. 761-770, 2011.

[53] N. Khabiri, S. Rast, and A. A. Senin, "Identifying Main Influential Elements in Technology Transfer Process: A Conceptual Model," Procedia - Soc. Behav. Sci., vol. 40, pp. 417-423, 2012.

[54] A. Pueyo, R. García, M. Mendiluce, and D. Morales, "The role of technology transfer for the development of a local wind component industry in Chile," Energy Policy, vol. 39, no. 7, pp. 4274-4283, 2011.

[55] E. G. Carayannis and J. Alexander, "Strategy, structure, and performance issues of precompetitive R D consortia: insights and lessons learned from SEMATECH," IEEE Trans. Eng. Manag., vol. 51, no. 2, pp. 226-232, May 2004.

[56] W. Gao and Q. Zhang, Research on Interactive Civil-Military Technology Transfer Platform Based on Knowledge Flow. Lancaster: Destech Publications, Inc, 2014.

[57] Y.-C. Hsu and C.-C. Lee, "The impact of military technology transfer on economic growth: international evidence," Appl. Econ., vol. 44, no. 19, pp. 2437-2449, 2012.

[58] Y. Zhang and Z. Jiang, The System Dynamics Model of Civil-military Technology Transfer in Defense Enterprise. Liverpool: World Acad Union-World Acad Press, 2008.

[59] R. M. Franza and K. P. Grant, "Improving Federal to Private Sector Technology Transfer," Res. Technol. Manag., vol. 49, no. 3, pp. 36-40, 2006.

[60] E. A. Isaacs and J. C. Tang, "Technology transfer: so much research, so few good products," Commun. ACM, vol. 39, no. 9, pp. 23-25, 1996.

[61] M. S. Spann, M. Adams, and W. E. Souder, "Measures of Technology Transfer Effectiveness: Key Dimensions and Differences in Their Use by Sponsors, Developers and Adopters," IEEE Trans. Eng. Manag., vol. 42, no. 1, pp. 19-29, 1995. 
[62] A. Chen, D. Patton, and M. Kenney, "University technology transfer in China: a literature review and taxonomy," J. Technol. Transf., vol. 41, no. 5, pp. 891929, Oct. 2016.

[63] D. S. Siegel, D. A. Waldman, L. E. Atwater, and A. N. Link, "Toward a model of the effective transfer of scientific knowledge from academicians to practitioners: Qualitative evidence from the commercialization of university technologies," $J$. Eng. Technol. Manag. - JET-M, vol. 21, no. 1-2, pp. 115-142, 2004.

[64] M. Schoonmaker, E. Carayannis, and P. Rau, "The role of marketing activities in the fuzzy front end of innovation: a study of the biotech industry," J. Technol. Transf., vol. 38, no. 6, pp. 850-872, Dec. 2013.

[65] G. Festel, "TECHNOLOGY TRANSFER MODELS BASED ON ACADEMIC SPINOFFS WITHIN THE INDUSTRIAL BIOTECHNOLOGY SECTOR,” Int. J. Innov. Manag., vol. 19, no. 04, p. 1550031, Aug. 2015.

[66] A.-P. Hameri, "Technology transfer between basic research and industry," Technovation, vol. 16, no. 2, pp. 51-92, Feb. 1996.

[67] F. Betz, "Basic research and technology transfer," Int. J. Technol. Manag., vol. 9, no. 5-7, pp. 784-796, 1994.

[68] K. Clark, J. Cavicchi, K. Jensen, R. Fitzgerald, A. Bennett, and S. P. Kowalski, "Patent data mining: A tool for accelerating HIV vaccine innovation," Vaccine, vol. 29, no. 24, pp. 4086-4093, May 2011.

[69] L. R. Vega-Gonzalez, N. Qureshi, O. V. Kolokoltsev, R. Ortega-Martinez, and S. Blesa, "Technology valuation of a scanning probe microscope developed at a university in a developing country," Technovation, vol. 30, no. 9-10, pp. 533539, Oct. 2010.

[70] L. R. Vega-Gonzalez and J. M. Saniger Blesa, "Valuation Methodology for Technology Developed at Academic R\&D Groups," J. Appl. Res. Technol., vol. 8, no. 1, pp. 26-43, Apr. 2010.

[71] F. A. Kulacki, "The education of mechanical engineers for the 21st century," Jsme Int. J. Ser. -Mech. Mater. Eng., vol. 39, no. 4, pp. 467-478, Oct. 1996.

[72] R. Lucic and R. Rohrer, "Undergraduate Field Applications Engineers - a Successful Experiment in Electronic Design Automation TechnologyTransfer," Ieee Trans. Educ., vol. 38, no. 3, pp. 261-265, Aug. 1995.

[73] J.-B. Park, T.-K. Ryu, and D. V. Gibson, "Facilitating public-to-private technology transfer through consortia: initial evidence from Korea," J. Technol. Transf., vol. 35, no. 2, pp. 237-252, Apr. 2010.

[74] J. L. Lin, S.-C. Fang, S.-R. Fang, and F.-S. Tsai, "Network embeddedness and technology transfer performance in R\&D consortia in Taiwan," Technovation, vol. 29, no. 11, pp. 763-774, Nov. 2009.

[75] H. E. Aldrich, M. K. Bolton, T. Baker, and T. Sasaki, "Information exchange and governance structures in US and Japanese R\&D consortia: institutional and organizational influences," IEEE Trans. Eng. Manag., vol. 45, no. 3, pp. 263275, Aug. 1998.

[76] T. Mongkolwai, A. Assavanig, C. Amnajsongsiri, T. W. Flegel, and A. Bhumiratana, "Technology transfer for small and medium soy sauce 
fermentation factories in Thailand: a consortium approach," Food Res. Int., vol. 30, no. 8, pp. 555-563, Oct. 1997.

[77] D. Gibson, C. Kehoe, and S. Lee, "Collaborative Research as a Function of Proximity, Industry, and Company - a Case-Study of an Research-andDevelopment Consortium," Ieee Trans. Eng. Manag., vol. 41, no. 3, pp. 255263, Aug. 1994.

[78] R. Smilor and D. Gibson, "Technology-Transfer in Multiorganizational Environments - the Case of Research-and-Development Consortia," Ieee Trans. Eng. Manag., vol. 38, no. 1, pp. 3-13, Feb. 1991.

[79] T. Machikita and Y. Ueki, "Measuring and Explaining Innovative Capability: Evidence from Southeast Asia," Asian Econ. Policy Rev., vol. 10, no. 1, pp. 152173, Jan. 2015.

[80] N. Komendantova and A. Patt, "Employment under vertical and horizontal transfer of concentrated solar power technology to North African countries," Renew. Sustain. Energy Rev., vol. 40, pp. 1192-1201, Dec. 2014.

[81] A. Mukherjee and Y. Tsai, "Multi-sourcing as an entry deterrence strategy," Int. Rev. Econ. Finance, vol. 25, pp. 108-112, Jan. 2013.

[82] S. Dinda and A. Mukherjee, "International Outsourcing, Tax, and Patent Protection," J. Public Econ. Theory, vol. 13, no. 1, pp. 139-154, Feb. 2011.

[83] P. Lin and K. Saggi, "Multinational firms, exclusivity, and backward linkages," J. Int. Econ., vol. 71, no. 1, pp. 206-220, Mar. 2007.

[84] H. Pack and K. Saggi, "Vertical technology transfer via international outsourcing," J. Dev. Econ., vol. 65, no. 2, pp. 389-415, Aug. 2001.

[85] 0. Granstrand and C. Oskarsson, "Technology Diversification in Mul-Tech Corporations," Ieee Trans. Eng. Manag., vol. 41, no. 4, pp. 355-364, Nov. 1994.

[86] W. Mitchell, "Using Academic Technology - Transfer Methods and Licensing Incidence in the Commercialization of American Diagnostic-Imaging Equipment Research, 1954-1988," Res. Policy, vol. 20, no. 3, pp. 203-216, Jun. 1991.

[87] B. Raz, G. Steinberg, and A. Ruina, "A Quantitative Model of TechnologyTransfer and Technological Catch-up - the Case of Developed-Countries," Technol. Forecast. Soc. Change, vol. 24, no. 1, pp. 31-44, 1983.

[88] S. Seres, E. Haites, and K. Murphy, "Analysis of technology transfer in CDM projects: An update," Energy Policy, vol. 37, no. 11, pp. 4919-4926, 2009.

[89] B. Raz and I. Assa, "A Model of Coupled Technology-Transfer - a Logistic Curve Approach," Technol. Forecast. Soc. Change, vol. 33, no. 3, pp. 251-265, May 1988.

[90] W.-H. Lai and C.-T. Tsai, "Analyzing influence factors of technology transfer using fuzzy set theory," Int. J. Innov. Technol. Manag., vol. 7, no. 1, pp. 71-87, 2010.

[91] G. Secundo, G. Secundo, C. De Beer, C. De Beer, G. Passiante, and G. Passiante, "Measuring university technology transfer efficiency: a maturity level approach," Meas. Bus. Excell., vol. 20, no. 3, pp. 42-54, 2016. 
[92] C. Genet, K. Errabi, and C. Gauthier, "Which model of technology transfer for nanotechnology? A comparison with biotech and microelectronics," Technovation, vol. 32, no. 3-4, pp. 205-215, Apr. 2012.

[93] S. Kypreos and H. Turton, "Climate change scenarios and Technology Transfer Protocols," Energy Policy, vol. 39, no. 2, pp. 844-853, 2011.

[94] F. Zhang and K. S. Gallagher, "Innovation and technology transfer through global value chains: Evidence from China's PV industry," Energy Policy, vol. 94, pp. 191-203, 2016.

[95] T. Okazaki and M. Yamaguchi, "Accelerating the transfer and diffusion of energy saving technologies steel sector experience-Lessons learned," Energy Policy, vol. 39, no. 3, pp. 1296-1304, 2011.

[96] L. Proskuryakova, D. Meissner, and P. Rudnik, "The use of technology platforms as a policy tool to address research challenges and technology transfer," J. Technol. Transf., vol. 42, no. 1, pp. 206-227, Feb. 2017.

[97] D. Jugend and S. L. da Silva, "Integration of R\&D and new product development: case studies of Brazilian high-tech firms," Int. J. Bus. Innov. Res., vol. 8, no. 4, pp. 422-439, Jan. 2014.

[98] E. G. Carayannis, D. Meissner, and A. Edelkina, “Targeted innovation policy and practice intelligence (TIP2E): concepts and implications for theory, policy and practice," J. Technol. Transf., vol. 42, no. 3, pp. 460-484, Jun. 2017.

[99] H. Chesbrough, W. Vanhaverbeke, and J. West, Open innovation: Researching a new paradigm. Oxford University Press on Demand, 2006.

[100] E. G. Carayannis, S. Sindakis, and C. Walter, "Business Model Innovation as Lever of Organizational Sustainability," J. Technol. Transf., vol. 40, no. 1, pp. 85-104, Feb. 2015.

[101] M. Del Giudice, E. G. Carayannis, and V. Maggioni, “Global knowledge intensive enterprises and international technology transfer: emerging perspectives from a quadruple helix environment," J. Technol. Transf., vol. 42, no. 2, pp. 229-235, Apr. 2017.

[102] D. Meissner and E. G. Carayannis, "Value generation from industry-science linkages in light of targeted open innovation," J. Knowl. Manag., vol. 21, no. 2, pp. 295-307, 2017.

[103] P. Balachandra, H. S. K. Nathan, and B. S. Reddy, "Commercialization of sustainable energy technologies," Renew. Energy, vol. 35, no. 8, pp. 18421851, 2010.

[104] S. Hess and R. Y. Siegwart, "R\&D Venture: proposition of a technology transfer concept for breakthrough technologies with R\&D cooperation: A case study in the energy sector," J. Technol. Transf., vol. 38, no. 2, pp. 153-179, 2013.

[105] S.-W. Hung, C.-C. Chang, and P.-C. Chen, "Technology commercialization in energy-smart industries," in Technology Management in the Energy Smart World (PICMET), 2011 Proceedings of PICMET'11:, 2011, pp. 1-9.

[106] E. G. Carayannis and J. Alexander, "Secrets of success and failure in commercialising US government R\&D laboratory technologies: a structured case study approach," Int. J. Technol. Manag., vol. 18, no. 3-4, pp. 246-269, 1999. 
[107] A. H. Rubenstein, "Models and metrics for the technology transfer process from federal labs to application and the market," in Management of Engineering \& Technology, 2009. PICMET 2009. Portland International Conference on, 2009, pp. 2760-2770.

[108] C. Karakosta, H. Doukas, and J. Psarras, “Technology transfer through climate change: Setting a sustainable energy pattern," Renew. Sustain. Energy Rev., vol. 14, no. 6, pp. 1546-1557, 2010.

[109] U. Able-Thomas, "Models of renewable energy technology transfer to developing countries," Renew. Energy, vol. 9, no. 1-4, pp. 1104-1107, 1996.

[110] K. Malek, E. Maine, and I. McCarthy, "A benchmark analysis of Canadian clean technology commercialization accelerators," in Technology Management for Emerging Technologies (PICMET), 2012 Proceedings of PICMET'12:, 2012, pp. 836-845.

[111] K. Malek, E. Maine, and I. P. McCarthy, "A typology of clean technology commercialization accelerators," J. Eng. Technol. Manag., vol. 32, pp. 26-39, 2014.

[112] H. Liu and D. Liang, "A review of clean energy innovation and technology transfer in China," Renew. Sustain. Energy Rev., vol. 18, pp. 486-498, 2013.

[113] C.-W. Hsu and P.-L. Chang, "Innovative evaluation model of emerging energy technology commercialization," Innovation, vol. 15, no. 4, pp. 476-483, 2013.

[114] A. Manoukian, H. R. HassabElnaby, and V. Odabashian, "A proposed framework for renewable energy technology commercialization and partnership synergy: A case study approach," Am. J. Bus., vol. 30, no. 2, pp. 147-174, 2015.

[115] K. Kristinsson and R. Rao, "Interactive learning or technology transfer as a way to catch-up? Analysing the wind energy industry in Denmark and India," Ind. Innov., vol. 15, no. 3, pp. 297-320, 2008.

[116] F. Urban, Y. Zhou, J. Nordensvard, and A. Narain, "Firm-level technology transfer and technology cooperation for wind energy between Europe, China and India: From North-South to South-North cooperation?," Energy Sustain. Dev., vol. 28, pp. 29-40, 2015.

[117] J. Blohmke, "Technology complexity, technology transfer mechanisms and sustainable development," Energy Sustain. Dev., vol. 23, pp. 237-246, 2014.

[118] A. Dinmohammadi and M. Shafiee, "Determination of the Most Suitable Technology Transfer Strategy for Wind Turbines Using an Integrated AHPTOPSIS Decision Model,” Energies, vol. 10, no. 5, p. 642, 2017.

[119] P. Klintenberg, F. Wallin, and L. C. Azimoh, "Successful technology transfer: What does it take?,” Appl. Energy, vol. 130, pp. 807-813, 2014.

[120] J. Phillips, K. Das, and P. Newell, "Governance and technology transfer in the Clean Development Mechanism in India," Glob. Environ. Change, vol. 23, no. 6, pp. 1594-1604, 2013.

[121] P. Sugandhavanija, S. Sukchai, N. Ketjoy, and S. Klongboonjit, “Determination of effective university-industry joint research for photovoltaic technology transfer (UIJRPTT) in Thailand," Renew. Energy, vol. 36, no. 2, pp. 600-607, 2011. 
[122] B. Biagini, L. Kuhl, K. S. Gallagher, and C. Ortiz, "Technology transfer for adaptation," Nat. Clim. Change, vol. 4, no. 9, p. 828, 2014.

[123] A. Kolk, "The role of international business in clean technology transfer and development," Clim. Policy, vol. 15, no. 1, pp. 170-176, 2015.

[124] N. Atikah, A. H. Al Ghabid, W. Sutopo, A. Purwanto, and M. Nizam, "Technical feasibility for technology commercialization of battery lithium ion," in Electrical Engineering and Computer Science (ICEECS), 2014 International Conference on, 2014, pp. 308-314.

[125] X. Cao, Z. Wen, J. Chen, and H. Li, "Contributing to differentiated technology policy-making on the promotion of energy efficiency technologies in heavy industrial sector: a case study of China," J. Clean. Prod., vol. 112, pp. 14861497, 2016.

[126] P. Salwan, "Technology Transfer and Technology Management in Strategic Systems," Def. Sci. J., vol. 55, no. 2, p. 141, 2005.

[127] J. Estep and T. Daim, "A framework for technology transfer potential assessment," in Management of Engineering and Technology (PICMET), 2016 Portland International Conference on, 2016, pp. 2846-2852.

[128] J. Estep and T. Daim, "Multiple Perspectives of Technology Transfer : Technology Transfer from Government Labs," pp. 2378-2391, 2013.

[129] R. M. Ham and D. C. Mowery, "Improving industry-government cooperative R\&D,” Issues Sci. Technol., vol. 11, no. 4, pp. 67-73, 1995.

[130] L. Field, "DOE Cooperative Research and Development Agreements — DOE Directives, Delegations, and Requirements," Department of Energy. [Online]. Available: https://www.directives.doe.gov/directives-documents/400series/0483.1-BOrder-A. [Accessed: 06-Aug-2017].

[131] B. Bozeman and S. Pandey, "Cooperative R\&D in government laboratories: comparing the US and Japan," Technovation, vol. 14, no. 3, pp. 145-159, 1994.

[132] T. D. Perry IV, "Ampulse corporation: A case study on technology transfer in us department of energy laboratories," National Renewable Energy Laboratory (NREL), Golden, CO., 2010.

[133] A. E. Cirone and J. Urpelainen, "Political market failure? The effect of government unity on energy technology policy in industrialized democracies," Technovation, vol. 33, no. 10, pp. 333-344, 2013.

[134] A. N. Chester, "Aligning technology with business strategy," Res.-Technol. Manag., vol. 37, no. 1, pp. 25-32, 1994.

[135] R. Phaal, C. Farrukh, R. Mitchell, and D. Probert, "Starting-up roadmapping fast," Res.-Technol. Manag., vol. 46, no. 2, pp. 52-59, 2003.

[136] N. Gindy, H. Arman, and S. Cavin, "Linking R\&D investment strategies to business needs: Strategic Technology Alignment Roadmapping (STAR)," in Management of Engineering \& Technology, 2009. PICMET 2009. Portland International Conference on, 2009, pp. 2455-2465.

[137] C. Jin, Y. Wei, H. Yubing, and others, "The Research on the Influence Factors for Fitness between Technology Strategy and Corporate Strategy," in Information Processing (ISIP), 2008 International Symposiums on, 2008, pp. 472-476. 
[138] C. A. C. Gama and D. Güemes-Castorena, "Project portfolio and strategic alignment through technology roadmapping for medium sized organizations and business units," in Management of Engineering \& Technology (PICMET), 2014 Portland International Conference on, 2014, pp. 76-87.

[139] M. B. Lyne, "Aligning R\&D with business strategy," Res.-Technol. Manag., vol. 46, no. 6, pp. 44-46, 2003.

[140] E. Loyarte et al., "Technology roadmapping (TRM) and strategic alignment for an applied research centre: a case study with methodological contributions," RD Manag., vol. 45, no. 5, pp. 474-486, Nov. 2015.

[141] D. R. Probert, C. J. Farrukh, and R. Phaal, "Technology roadmappingdeveloping a practical approach for linking resources to strategic goals," Proc. Inst. Mech. Eng. Part B J. Eng. Manuf., vol. 217, no. 9, pp. 1183-1195, 2003.

[142] R. Wells, R. Phaal, C. Farrukh, and D. Probert, "Technology roadmapping for a service organization," Res.-Technol. Manag., vol. 47, no. 2, pp. 46-51, 2004.

[143] K. F. Herfert and M. V. Arbige, "Aligning an R\&D portfolio with corporate strategy," Res.-Technol. Manag., vol. 51, no. 5, pp. 39-46, 2008.

[144] P. M. Bosch-Sijtsema and J. Bosch, "Aligning innovation ecosystem strategies with internal R\&D," in Management of Innovation and Technology (ICMIT), 2014 IEEE International Conference on, 2014, pp. 424-430.

[145] P. L. Pereira and F. M. Veloso, "R\&D activity selection process: Building a strategy-aligned R\&D portfolio for government and nonprofit organizations," IEEE Trans. Eng. Manag., vol. 56, no. 1, pp. 95-105, 2009.

[146] N. Gindy, M. Morcos, B. Cerit, and A. Hodgson, "Strategic technology alignment roadmapping STAR $\AA$ aligning $R \& D$ investments with business needs," Int. J. Comput. Integr. Manuf., vol. 21, no. 8, pp. 957-970, 2008.

[147] S. T. Walsh and J. D. Linton, "The strategy-technology firm fit audit: a guide to opportunity assessment and selection," Technol. Forecast. Soc. Change, vol. 78, no. 2, pp. 199-216, 2011.

[148] R. Phaal, C. J. P. Farrukh, and D. R. Probert, "Technology roadmapping-A planning framework for evolution and revolution," Technol. Forecast. Soc. Change, vol. 71, no. 1-2, pp. 5-26, Jan. 2004.

[149] T. A. Kappel, "Perspectives on roadmaps: how organizations talk about the future," J. Prod. Innov. Manag., vol. 18, no. 1, pp. 39-50, 2001.

[150] J. L. M. de Carvalhoa and J. C. de Toledob, "The challenge of integrating applied research and product development process: Cases study," Prod. Manag. Dev., vol. 6, no. 2, 2008.

[151] P. Jervis, "Innovation and technology transfer-The roles and characteristics of individuals," IEEE Trans. Eng. Manag., no. 1, pp. 19-27, 1975.

[152] Y. Aoshima, "System-based improvement and knowledge transfer across multiple generations of product development project," Int. Mot. Veh. Program Pap. Sloan Sch. Manag. MIT Massachusset, 1994.

[153] J. Scholtz, "Technology transfer through prototypes," Commun. ACM, vol. 39, no. 9, pp. 26-28, 1996.

[154] I. Nonaka and H. Takeuchi, The knowledge-creating company: How Japanese companies create the dynamics of innovation. Oxford university press, 1995. 
[155] E. G. Carayannis, J. Alexander, and A. Ioannidis, "Leveraging knowledge, learning, and innovation in forming strategic government-universityindustry (GUI) R\&D partnerships in the US, Germany, and France," Technovation, vol. 20, no. 9, pp. 477-488, Sep. 2000.

[156] P. Reason and H. Bradbury, Handbook of action research: Concise paperback edition. Sage, 2005.

[157] D. Chandler and B. Torbert, "Transforming inquiry and action interweaving 27 flavors of action research," Action Res., vol. 1, no. 2, pp. 133-152, 2003.

[158] E. Ferrance, Action research. LAB, Northeast and Island Regional Education Laboratory at Brown University, 2000.

[159] R. O’Brien, "An overview of the methodological approach of action research," Fac. Inf. Stud. Univ. Tor., 1998.

[160] D. Tripp, “Action research: a methodological introduction,” Educ. E Pesqui., vol. 31, no. 3, pp. 443-466, 2005.

[161] G. I. Susman and R. D. Evered, "An assessment of the scientific merits of action research," Adm. Sci. Q., pp. 582-603, 1978.

[162] J. Martí, "Measuring in action research: Four ways of integrating quantitative methods in participatory dynamics," Action Res., vol. 14, no. 2, pp. 168-183, 201606.

[163] D. Dick and B. Dick, "Reflections on the SAGE Encyclopedia of Action Research and what it says about action research and its methodologies," Action Res., vol. 13, no. 4, pp. 431-444, 201512.

[164] A. O. Davidson, "Observing action research processes in practice," The University of North Carolina at Chapel Hill, 2009.

[165] J. Village, M. Greig, F. Salustri, S. Zolfaghari, and W. P. Neumann, “An ergonomics action research demonstration: integrating human factors into assembly design processes," Ergonomics, vol. 57, no. 10, pp. 1574-1589, 2014.

[166] B. Dick and D. Greenwood, "Theory and method: Why action research does not separate them," Action Res., vol. 13, no. 2, pp. 194-197, 201506.

[167] P. McManners, "The action research case study approach: A methodology for complex challenges such as sustainability in aviation," Action Res., vol. 14, no. 2, pp. 201-216, 201606.

[168] M. Perona, N. Saccani, S. Bonetti, and A. Bacchetti, "Manufacturing lead time shortening and stabilisation by means of workload control: an action research and a new method," Prod. Plan. Control, vol. 27, no. 7-8, pp. 660-670, 2016.

[169] R. Phaal, C. J. P. Farrukh, and D. R. Probert, "Technology management process assessment: a case study," Int. J. Oper. Prod. Manag., vol. 21, no. 8, pp. 11161132, 2001.

[170] S. Ottosson, "Participation action research - A key to improved knowledge of management," Technovation, vol. 23, no. 2, pp. 87-94, Feb. 2003.

[171] F. Faure, H. Hocdé, and E. Chia, "Action research methodology to reconcile product standardization and diversity of agricultural practices: A case of farmers' organizations in Costa Rica," Action Res., vol. 9, no. 3, pp. 242-260, 201109. 
[172] M. Moschitz and R. Home, "The challenges of innovation for sustainable agriculture and rural development: Integrating local actions into European policies with the Reflective Learning Methodology," Action Res., vol. 12, no. 4, pp. 392-409, 201412.

[173] C. Arcidiacono, D. Grimaldi, S. Di Martino, and F. Procentese, "Participatory visual methods in the 'Psychology loves Porta Capuana' project," Action Res., vol. 14, no. 4, pp. 376-392, 201612.

[174] M. Ingram, L. Murrietta, J. G. de Zapien, P. M. Herman, and S. C. Carvajal, "Community health workers as focus group facilitators: A participatory action research method to improve behavioral health services for farmworkers in a primary care setting," Action Res., vol. 13, no. 1, pp. 48-64, 201503.

[175] V. Nyman, M. Berg, S. Downe, and T. Bondas, "Insider Action research as an approach and a method - Exploring institutional encounters from within a birthing context," Action Res., vol. 14, no. 2, pp. 217-233, 201606.

[176] C. Rydenfält, P.-A. Larsson, and P. Odenrick, "An action-oriented method for interprofessional organization development at a hospital operating unit," Action Res., vol. 15, no. 2, pp. 177-197, 2017.

[177] J. R. Voigt, U. M. Hansen, M. Glindorf, R. Poulsen, and I. Willaing, "Action research as a method for changing patient education practice in a clinical diabetes setting," Action Res., vol. 12, no. 3, pp. 315-336, 2014.

[178] D. Clover, "Successes and challenges of feminist arts-based participatory methodologies with homeless/street-involved women in Victoria," Action Res., vol. 9, no. 1, pp. 12-26, 201103.

[179] K. Viikki and J. Palviainen, "Integrating human-centered design into software development: An action research study in the automation industry," in Software Engineering and Advanced Applications (SEAA), 2011 37th EUROMICRO Conference on, 2011, pp. 313-320.

[180] G. Duncan and R. Ridley-Duff, "Appreciative Inquiry as a method of transforming identity and power in Pakistani women," Action Res., vol. 12, no. 2, pp. 117-135, 201406.

[181] A. Arantes, L. M. D. Ferreira, and A. A. Kharlamov, "Application of a purchasing portfolio model in a construction company in two distinct markets," J. Manag. Eng., vol. 30, no. 5, p. 04014020, 2014.

[182] C.-Y. Shen and G. Midgley, "Action research in a problem avoiding culture using a Buddhist systems methodology," Action Res., vol. 13, no. 2, pp. 170193, 201506.

[183] L. Gerassi, T. Edmond, and A. Nichols, "Design strategies from sexual exploitation and sex work studies among women and girls: Methodological considerations in a hidden and vulnerable population," Action Res., p. 1476750316630387, 2016.

[184] B. R. Kattman, "An action research study; cultural differences impact how manufacturing organizations receive continuous improvement," The National Graduate School of Quality Management, 2012. 
[185] P. K. Dey, A. Bhattacharya, and W. Ho, "Strategic supplier performance evaluation: A case-based action research of a UK manufacturing organisation," Int. J. Prod. Econ., vol. 166, pp. 192-214, 2015.

[186] T. Schoenherr, V. R. Tummala, and T. P. Harrison, “Assessing supply chain risks with the analytic hierarchy process: Providing decision support for the offshoring decision by a US manufacturing company," J. Purch. Supply Manag., vol. 14, no. 2, pp. 100-111, 2008.

[187] A. Small and D. Wainwright, "SSM and technology management: Developing multimethodology through practice," Eur. J. Oper. Res., vol. 233, no. 3, pp. 660673, Mar. 2014.

[188] T. Waring, L. Johnston, A. McGrane, T. Nguyen, and P. Scullion, "Developing knowledge sharing partnerships in the SME sector: an action research approach," in European Conference on Research Methodology for Business and Management Studies, 2013, p. 354.

[189] P. T. Houngbo, H. L. S. Coleman, M. Zweekhorst, T. D. C. Buning, D. Medenou, and J. F. G. Bunders, "A Model for Good Governance of Healthcare Technology Management in the Public Sector: Learning from Evidence-Informed Policy Development and Implementation in Benin," Plos One, vol. 12, no. 1, p. e0168842, Jan. 2017.

[190] R. K. Yin, Case study research: Design and methods. Sage publications, 2013.

[191] W. Ellet, The case study handbook: How to read, discuss, and write persuasively about cases. Harvard Business Press, 2007.

[192] R. Klonoski, "The case for case studies: deriving theory from evidence," J. Bus. Case Stud. Online, vol. 9, no. 3, p. 261, 2013.

[193] S. Jun, S. Park, and D. Jang, "A Technology Valuation Model Using Quantitative Patent Analysis: A Case Study of Technology Transfer in Big Data Marketing," Emerg. Mark. Finance Trade, vol. 51, no. 5, pp. 963-974, Oct. 2015.

[194] Y. Servati, S. H. Ghodsypour, and M. A. Shirazi, "Robust Technology Transfer Policy Making Using Scenario Based Fuzzy Topsis - a Case Study of Iran's Gas Industry," Appl. Ecol. Environ. Res., vol. 15, no. 3, pp. 593-610, 2017.

[195] G. M. do Canto Cavalheiro and L. A. Joia, "E-Government Technology Transfer: A Case Study of the Implementation of a European Patent Management System in Brazil," Public Adm. Dev., vol. 36, no. 3, pp. 215-231, Aug. 2016.

[196] J. Nakhaei, M. Bitarafan, and S. L. Arefi, "Choosing the Best Urban Tunnels as Safe Space in Crisis Using Ahp Method: A Case Study in Iran," J. Archit. Urban., vol. 39, no. 2, pp. 149-160, 2015.

[197] B. Wang, "Can CDM bring technology transfer to China?-An empirical study of technology transfer in China's CDM projects," Energy Policy, vol. 38, no. 5, pp. 2572-2585, 2010.

[198] Y. O. Ouma, J. Opudo, and S. Nyambenya, "Comparison of Fuzzy AHP and Fuzzy TOPSIS for Road Pavement Maintenance Prioritization: Methodological Exposition and Case Study," Adv. Civ. Eng., p. 140189, 2015.

[199] W. Wang et al., "The design and implementation of risk assessment model for hazard installations based on AHP-FCE method: A case study of Nansi Lake Basin," Ecol. Inform., vol. 36, pp. 162-171, Nov. 2016. 
[200] X. Zhang, L. Kuchinke, M. L. Woud, J. Velten, and J. Margraf, "Survey method matters: Online/offline questionnaires and face-to-face or telephone interviews differ," Comput. Hum. Behav., vol. 71, pp. 172-180, Jun. 2017.

[201] I. Seidman, Interviewing as Qualitative Research: A Guide for Researchers in Education and the Social Sciences. Teachers College Press, 2013.

[202] R. S. Weiss, Learning from strangers. New York: The Free Press, 1994.

[203] E. Ash, "Motivational Interviewing in the Reciprocal Engagement Model of Genetic Counseling: a Method Overview and Case Illustration," J. Genet. Couns., vol. 26, no. 2, pp. 300-311, Apr. 2017.

[204] J. S. Baer, D. B. Rosengren, C. W. Dunn, E. A. Wells, R. L. Ogle, and B. Hartzler, "An evaluation of workshop training in motivational interviewing for addiction and mental health clinicians," Drug Alcohol Depend., vol. 73, no. 1, pp. 99-106, Jan. 2004.

[205] J. S. Baer, E. A. Wells, D. B. Rosengren, B. Hartzler, B. Beadnell, and C. Dunn, "Agency context and tailored training in technology transfer: A pilot evaluation of motivational interviewing training for community counselors," J. Subst. Abuse Treat., vol. 37, no. 2, pp. 191-202, Sep. 2009.

[206] C. Dunn et al., "Within-Provider Variability in Motivational Interviewing Integrity for Three Years after MI Training: Does Time Heal?," J. Subst. Abuse Treat., vol. 65, pp. 74-82, Jun. 2016.

[207] S. Martino, S. A. Ball, C. Nich, M. Canning-Ball, B. J. Rounsaville, and K. M. Carroll, "Teaching community program clinicians motivational interviewing using expert and train-the-trainer strategies," Addiction, vol. 106, no. 2, pp. 428-441, Feb. 2011.

[208] M. S. Shafer, R. Rhode, and J. Chong, "Using distance education to promote the transfer of motivational interviewing skills among behavioral health professionals," J. Subst. Abuse Treat., vol. 26, no. 2, pp. 141-148, Mar. 2004.

[209] P. Haoshu, Y. Jianhua, and Y. Xiaoliang, "How do Chinese Enterprises Conduct Technology Innovation? Implications from Interviews to 25 NationAuthorized Enterprise Technology Centers," in 2008 Portland International Conference on Management of Engineering \& Technology, Vols 1-5, New York: Ieee, 2008, pp. 2296-2304.

[210] R. C. Veniegas, U. H. Kao, and R. Rosales, "Adapting HIV prevention evidencebased interventions in practice settings: an interview study," Implement. Sci., vol. 4, p. 76, Nov. 2009.

[211] P. Chaudhry, B. Singh, and V. P. Tewari, "Non-market economic valuation in developing countries: Role of participant observation method in CVM analysis," J. For. Econ., vol. 13, no. 4, pp. 259-275, 2007.

[212] J. P. Spradley, Participant Observation. Fort Worth: Holt, Rinehart, and Winston. Inc, 1980.

[213] M. S. Schwartz and C. G. Schwartz, "Problems in participant observation," Am. J. Sociol., vol. 60, no. 4, pp. 343-353, 1955.

[214] E. Latvala, P. Vuokila-Oikkonen, and S. Janhonen, "Videotaped recording as a method of participant observation in psychiatric nursing research," J. Adv. Nurs., vol. 31, no. 5, pp. 1252-1257, May 2000. 
[215] A. James, "Learning to be friends - Methodological lessons from participant observation among English schoolchildren," Child.- Glob. J. Child Res., vol. 3, no. 3, pp. 313-330, Aug. 1996.

[216] C. Crocker and S. Timmons, “The role of technology in critical care nursing," J. Adv. Nurs., vol. 65, no. 1, pp. 52-61, Jan. 2009.

[217] Z. Glabinski, "Analysing the tourism activity of seniors by applying the method of participant observation," Bull. Geogr.-Socio-Econ. Ser., vol. 33, no. 33, pp. 55-70, Sep. 2016.

[218] K. Itaya and K. Niwa, "Trial implementation of a highly autonomous smallteam-type R\&D management model in a Japanese electronics company," Int. J. Technol. Manag., vol. 53, no. 2-4, pp. 273-288, 2011.

[219] E. Colucci, "'Focus groups can be fun': The use of activity-oriented questions in focus group discussions," Qual. Health Res., vol. 17, no. 10, pp. 1422-1433, 2007.

[220] J. Cyr, "The Pitfalls and Promise of Focus Groups as a Data Collection Method," Sociol. Methods Res., vol. 45, no. 2, pp. 231-259, May 2016.

[221] E. Risco, E. Cabrera, M. Farre, C. Alvira, S. Miguel, and A. Zabalegui, "Perspectives About Health Care Provision in Dementia Care in Spain: A Qualitative Study Using Focus-Group Methodology," Am. J. Alzheimers Dis. Other Demen., vol. 31, no. 3, pp. 223-230, May 2016.

[222] E. Beukman and H. Steyn, "Phasing Technology Transfer Projects for Sustainable Socio-Economic Development," South Afr. J. Ind. Eng., vol. 22, no. 2, pp. 40-53, Nov. 2011.

[223] T. L. Greenbaum, The handbook for focus group research. Sage, 1998.

[224] T. L. Greenbaum, Moderating focus groups: A practical guide for group facilitation. Sage Publications, 1999.

[225] M. Greenwood, T. Kendrick, H. Davies, and F. J. Gill, "Hearing voices: Comparing two methods for analysis of focus group data," Appl. Nurs. Res., vol. 35, pp. 90-93, Jun. 2017.

[226] R. A. Krueger and M. A. Casey, Focus groups: A practical guide for applied research. Sage publications, 2014.

[227] G. Abdollahzadeh, C. A. Damalas, M. S. Sharifzadeh, and H. Ahmadi-Gorgi, "Selecting strategies for rice stem borer management using the Analytic Hierarchy Process (AHP)," Crop Prot., vol. 84, pp. 27-36, Jun. 2016.

[228] H. X. Li, M. Al-Hussein, Z. Lei, and Z. Ajweh, "Risk identification and assessment of modular construction utilizing fuzzy analytic hierarchy process (AHP) and simulation," Can. J. Civ. Eng., vol. 40, no. 12, pp. 1184-1195, Dec. 2013.

[229] P. S. Sarathy, "TQM practice in real-estate industry using AHP," Qual. Quant., vol. 47, no. 4, pp. 2049-2063, Jun. 2013.

[230] A. K. Singh, "Competitive service quality benchmarking in airline industry using AHP," Benchmarking- Int. J., vol. 23, no. 4, pp. 768-791, 2016.

[231] Y. Liu, S. Van Nederveen, and M. Hertogh, "Understanding effects of BIM on collaborative design and construction: An empirical study in China," Int. J. Proj. Manag., vol. 35, no. 4, pp. 686-698, May 2017. 
[232] K. Jacklin, A. Ly, B. Calam, M. Green, L. Walker, and L. Crowshoe, "An Innovative Sequential Focus Group Method for Investigating Diabetes Care Experiences With Indigenous Peoples in Canada," Int. J. Qual. Methods, vol. 15, no. 1, Dec. 2016.

[233] M. Dubickis and E. Gaile-Sarkane, "Perspectives on Innovation and Technology Transfer," in 20th International Scientific Conference - Economics and Management 2015 (icem-2015), vol. 213, E. Gimzauskiene, K. Duoba, X. Pavie, A. Pinnington, and M. Vilka, Eds. Amsterdam: Elsevier Science Bv, 2015, pp. 965-970.

[234] N. Nahar, K. Lyytinen, N. Huda, and S. V. Muravyov, "Success factors for information technology supported international technology transfer: Finding expert consensus," Inf. Manage., vol. 43, no. 5, pp. 663-677, Jul. 2006.

[235] D. F. Kocaoglu, "A Participative Approach to Program Evaluation,” no. 3, pp. 112-118, 1983.

[236] S. Munkongsujarit et al., "Decision model for a place to live at PSU: The case of international graduate students," PICMET 09 - 2009 Portland Int. Conf. Manag. Eng. Technol., pp. 513-534, Aug. 2009.

[237] R. A. Taha, B. C. Choi, P. Chuengparsitporn, A. Cutar, Q. Gu, and K. Phan, "Application of Hierarchical Decision Modeling for Selection of Laptop," PICMET 07 - 2007 Portland Int. Conf. Manag. Eng. Technol., pp. 1160-1175, Aug. 2007.

[238] K. Phan and D. F. Kocaoglu, "Innovation Measurement Framework to Determine Innovativeness of a Company : Case of Semiconductor Industry," pp. 747-757, 2014.

[239] N. J. Sheikh, Y. Park, and D. F. Kocaoglu, "Assessment of Solar Photovoltaic Technologies Using Multiple Perspectives and Hierarchical Decision Modeling : Manufacturers Worldview," pp. 491-497, 2014.

[240] T. Turan, M. Amer, P. Tibbot, M. Almasri, F. Al Fayez, and S. Graham, "Use of Hierarchal Decision Modeling (HDM) for selection of graduate school for master of science degree program in engineering," PICMET 09 - 2009 Portland Int. Conf. Manag. Eng. Technol., pp. 535-549, Aug. 2009.

[241] P. Gerdsri and D. F. Kocaoglu, "HDM for Developing N ational Emerging Technology Strategy and Policy Supporting Sustainable Economy : A Case Study of N anotechnology for Thailand 's Agriculture," no. c, pp. 27-31, 2008.

[242] J. Thompson, B. Barnwell, T. Calderwood, A. Kumar, and S. Vang, "Decision Model for Portland Metro Bike Commuters," 2011.

[243] L. Hogaboam, B. Ragel, and T. Daim, "Development of a Hierarchical Decision Model ( HDM ) for Health Technology Assessment ( HTA ) to Design and Implement a New Patient Care Database for Low Back Pain," pp. 3511-3517, 2014.

[244] B. Wang, D. F. Kocaoglu, T. U. Daim, and J. Yang, "A decision model for energy resource selection in China," Energy Policy, vol. 38, no. 11, pp. 7130-7141, Nov. 2010. 
[245] J. Estep, “Development of a Technology Transfer Score for Evaluating Research Proposals: Case Study of Demand Response Technologies in the Pacific Northwest," Portland State University, 2017.

[246] H. Chen and D. F. Kocaoglu, "A sensitivity analysis algorithm for hierarchical decision models," Eur. J. Oper. Res., vol. 185, no. 1, pp. 266-288, Feb. 2008.

[247] T. L. Saaty, "The analytic hierarchy process: planning," Prior. Setting Resour. Alloc. MacGraw-Hill N. Y. Int. Book Co., p. 287, 1980.

[248] M. Abbas, "Consistency Analysis for Judgment Quantification in Hierarchical Decision Model," Diss. Theses, Mar. 2016.

[249] M. S. Abbas and D. F. Kocaoglu, "Consistency Thresholds for Hierarchical Decision Model," in Portland International Conference on Management of Engineering and Technology (picmet 2016): Technology Management for Social Innovation, D. F. Kocaoglu, T. R. Anderson, T. U. Daim, D. C. Kozanoglu, K. Niwa, and G. Perman, Eds. New York: Ieee, 2016, pp. 566-575.

[250] D. F. Kocaoglu, "A Participative Approach to Program Evaluation," no. 3, pp. 112-118, 1983.

[251] T. L. Saaty, "A scaling method for priorities in hierarchical structures," J. Math. Psychol., vol. 15, no. 3, pp. 234-281, 1977.

[252] P. Gerdsri and D. Kocaoglu, "A Systematic Approach to Developing 1ational Technology Policy and Strategy for Emerging Technologies : A Case Study of 1anotechnology for Thailand 's Agriculture Industry," pp. 447-461, 2009.

[253] R. D. Holder, "Some comments on the analytic hierarchy process," J. Oper. Res. Soc., vol. 41, no. 11, pp. 1073-1076, 1990.

[254] C. K. Murphy, "Limits on the analytic hierarchy process from its consistency index," Eur. J. Oper. Res., vol. 65, no. 1, pp. 138-139, 1993.

[255] "expert Definition in the Cambridge English Dictionary," Cambridge Dictionary. [Online]. Available:

http://dictionary.cambridge.org/us/dictionary/english/expert. [Accessed: 08-Sep-2017].

[256] “Definition of PANEL," Merriam-Webster Dictionary. [Online]. Available: https://www.merriam-webster.com/dictionary/panel. [Accessed: 08-Sep2017].

[257] H. L. Dreyfus and S. E. Dreyfus, "Peripheral vision: Expertise in real world contexts," Organ. Stud., vol. 26, no. 5, pp. 779-792, 2005.

[258] C. Evans, "The use of consensus methods and expert panels in pharmacoeconomic studies," Pharmacoeconomics, vol. 12, no. 2, pp. 121-129, 1997.

[259] D. Holman et al., "Change and innovation in modern manufacturing practices: An expert panel survey of U. K. companies," Hum. Factors Ergon. Manuf., vol. 10, no. 2, pp. 121-137, 2000.

[260] A. B. Knol, P. Slottje, J. P. van der Sluijs, and E. Lebret, "The use of expert elicitation in environmental health impact assessment: a seven step procedure," Environ. Health, vol. 9, no. 1, p. 19, 2010. 
[261] M. G. Morgan, "Use (and abuse) of expert elicitation in support of decision making for public policy," Proc. Natl. Acad. Sci., vol. 111, no. 20, pp. 71767184, 2014.

[262] G. F. Nemet, L. D. Anadon, and E. Verdolini, "Quantifying the effects of expert selection and elicitation design on experts' confidence in their judgments about future energy technologies," Risk Anal., vol. 37, no. 2, pp. 315-330, 2017.

[263] R. Abotah, "Evaluation of Energy Policy Instruments for the Adoption of Renewable Energy: Case of Wind Energy in the Pacific Northwest U.S," Ph.D., Portland State University, United States -- Oregon, 2015.

[264] T. A. Tran, "Strategic Evaluation of University Knowledge and Technology Transfer Effectiveness," Portland State University, 2013.

[265] K. Phan, "Innovation measurement: A decision framework to determine innovativeness of a company," Portland State University, 2013.

[266] L. Chan, "Developing a Strategic Policy Choice Framework for Technological Innovation: Case of Chinese Pharmaceuticals," Portland State University, 2013.

[267] I. Iskin, “An Assessment Model for Energy Efficiency Program Planning in Electric Utilities: Case of the Pacific of Northwest U.S.A.," Diss. Theses, Jun. 2014.

[268] E. C. Gibson, “A Measurement System for Science and Engineering Research Center Performance Evaluation," 2016.

[269] R. Trevethan, "Intraclass correlation coefficients: clearing the air, extending some cautions, and making some requests," Health Serv. Outcomes Res. Methodol., vol. 17, no. 2, pp. 127-143, 2017.

[270] J. M. LeBreton and J. L. Senter, "Answers to 20 questions about interrater reliability and interrater agreement," Organ. Res. Methods, vol. 11, no. 4, pp. 815-852, 2008.

[271] D. J. Sheskin, Handbook of parametric and nonparametric statistical procedures. crc Press, 2003.

[272] Andale, "F Test: Definition, Examples, How to Run One," Statistics How To. [Online]. Available: http://www.statisticshowto.com/f-test/. [Accessed: 09Sep-2017].

[273] P. E. Shrout and J. L. Fleiss, "Intraclass correlations: uses in assessing rater reliability.," Psychol. Bull., vol. 86, no. 2, p. 420, 1979.

[274] H. Chen and D. F. Kocaoglu, "A sensitivity analysis algorithm for hierarchical decision models," Eur. J. Oper. Res., vol. 185, no. 1, pp. 266-288, Feb. 2008.

[275] H. Chen, "Sensitivity analysis for hierarchical decision models," Ph.D., Portland State University, United States -- Oregon, 2007.

[276] B. Yildiz, "Assessment of Policy Alternatives for Mitigation of Barriers to EV Adoption," Portland State University, Portland, Oregon, 2018.

[277] J. Lavoie, J. Kim, and T. Daim, "A Technology Transfer Framework: A Case Study from the Energy Sector," Int. J. Innov. Manag. Technol., vol. 8, no. 4, pp. 249-260, Aug. 2017. 


\section{APPENDIX A - RSV CALCULATION}

Given the 120 orientations (five variables, 5!), a random stratified sample of 50 orientations was chosen to calculate the inconsistency of expert 36 . The figure below shows the sample.

\begin{tabular}{|c|c|c|c|c|}
\hline AEBDC & BECDA & CBDAE & DEACB & EBCDA \\
\hline ACEDB & BDCAE & CBEAD & DECAB & EBCAD \\
\hline ADBCE & BCADE & CEABD & DBECA & EBDAC \\
\hline ACDEB & BDECA & CEDBA & DEBAC & EDCBA \\
\hline ABCED & BACDE & CBEDA & DABCE & EACBD \\
\hline ADCEB & BCAED & CBADE & DCEAB & EADCB \\
\hline ABDEC & BADEC & CEBAD & DBCEA & ECBDA \\
\hline AEDCB & BDACE & CBAED & DBCAE & EADBC \\
\hline ADEBC & BCDAE & CADEB & DABEC & EBACD \\
\hline AECDB & BADCE & CBDEA & DCAEB & ECDBA \\
\hline
\end{tabular}

After calculating matrices A, B, and C for each of the orientations within the sample, the orientations table was built, as shown in the next figure.

Before Normalization

\begin{tabular}{|c|c|c|c|c|c|c|c|}
\hline$\#$ & Orientation & $\mathrm{A}$ & $\mathrm{B}$ & $\mathrm{C}$ & $\mathrm{D}$ & $\mathrm{E}$ & Sum \\
\hline 1 & AEBDC & 2.29 & 1.20 & 1.00 & 2.14 & 5.80 & 12.43 \\
\hline 2 & ACEDB & 1.20 & 1.00 & 2.14 & 2.29 & 0.38 & 7.00 \\
\hline 3 & ADBCE & 7.72 & 0.59 & 1.30 & 2.69 & 1.00 & 13.30 \\
\hline 4 & ACDEB & 3.79 & 1.00 & 2.66 & 0.88 & 5.80 & 14.13 \\
\hline 5 & ABCED & 7.62 & 0.59 & 1.30 & 1.00 & 5.20 & 15.71 \\
\hline 6 & ADCEB & 7.72 & 1.00 & 1.30 & 2.14 & 5.80 & 17.96 \\
\hline 7 & ABDEC & 7.62 & 1.20 & 1.00 & 0.88 & 2.70 & 13.40 \\
\hline 8 & AEDCB & 2.29 & 1.00 & 2.08 & 2.14 & 5.20 & 12.71 \\
\hline 9 & ADEBC & 7.72 & 0.59 & 1.00 & 0.88 & 5.80 & 15.99 \\
\hline 10 & AECDB & 2.29 & 1.00 & 2.66 & 2.69 & 2.70 & 11.34 \\
\hline 11 & BECDA & 1.00 & 1.00 & 2.66 & 0.41 & 2.70 & 7.77 \\
\hline 12 & BDCAE & 2.29 & 1.20 & 0.38 & 2.14 & 1.00 & 7.01 \\
\hline 13 & BCADE & 7.72 & 0.59 & 0.38 & 0.88 & 1.00 & 10.57 \\
\hline
\end{tabular}

Normalized Values

\begin{tabular}{|c|c|c|c|c|}
\hline$A$ & $B$ & $C$ & $D$ & $E$ \\
\hline 0.1842 & 0.0965 & 0.0805 & 0.1722 & 0.4666 \\
\hline 0.1720 & 0.1429 & 0.3050 & 0.3264 & 0.0537 \\
\hline 0.5805 & 0.0444 & 0.0977 & 0.2023 & 0.0752 \\
\hline 0.2682 & 0.0708 & 0.1883 & 0.0623 & 0.4105 \\
\hline 0.4850 & 0.0376 & 0.0827 & 0.0637 & 0.3310 \\
\hline 0.4298 & 0.0557 & 0.0724 & 0.1192 & 0.3229 \\
\hline 0.5687 & 0.0896 & 0.0746 & 0.0657 & 0.2015 \\
\hline 0.1802 & 0.0787 & 0.1637 & 0.1684 & 0.4091 \\
\hline 0.4828 & 0.0369 & 0.0625 & 0.0550 & 0.3627 \\
\hline 0.2019 & 0.0882 & 0.2346 & 0.2372 & 0.2381 \\
\hline 0.1287 & 0.1287 & 0.3423 & 0.0528 & 0.3475 \\
\hline 0.3267 & 0.1712 & 0.0542 & 0.3053 & 0.1427 \\
\hline 0.7304 & 0.0558 & 0.0360 & 0.0833 & 0.0946 \\
\hline
\end{tabular}




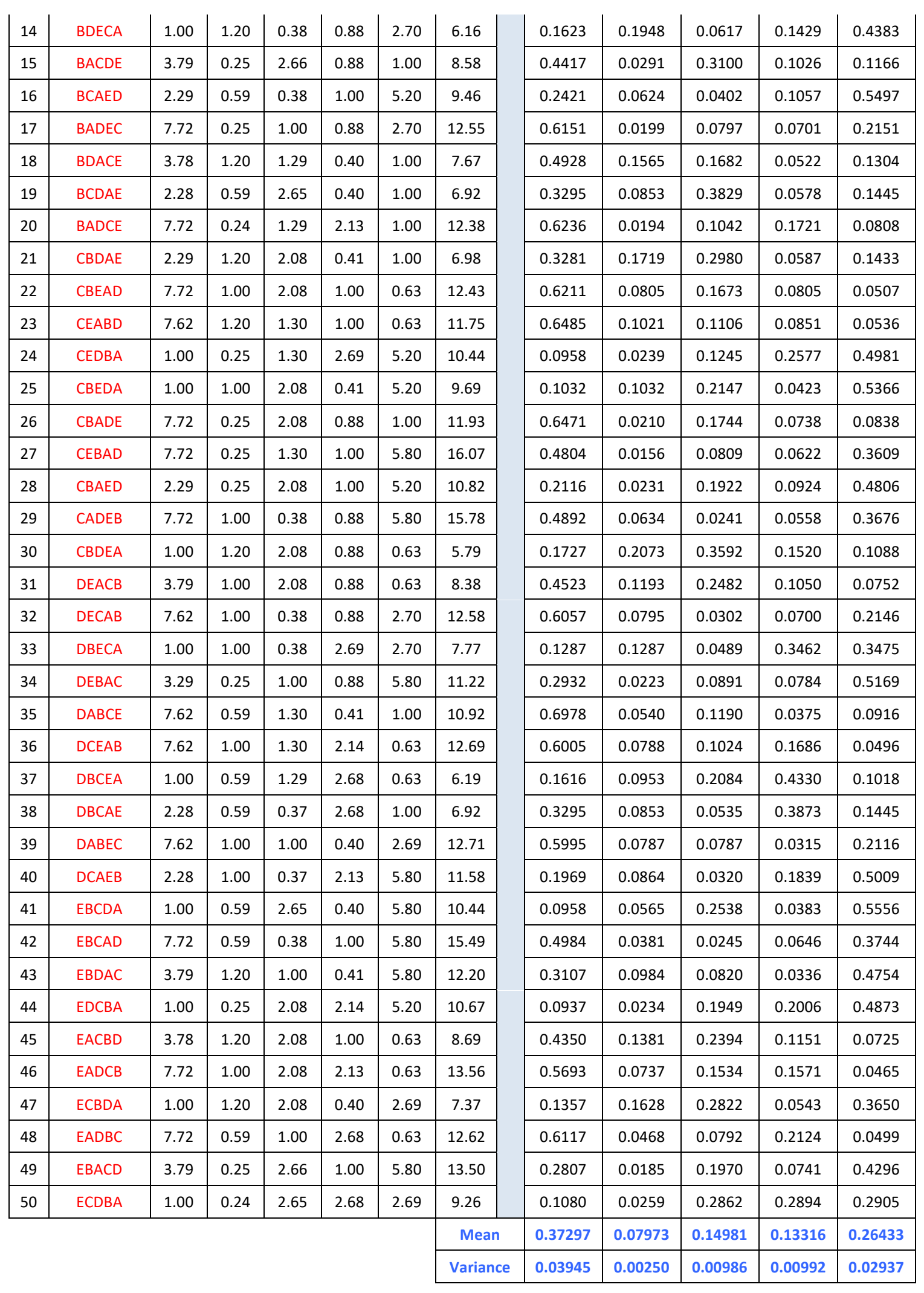


The RSV was then calculated:

$$
\begin{gathered}
R S V=\sqrt{\sum_{1=1}^{n} \sigma_{n}^{2}} \\
R S V=\sqrt{(0.03945+0.00250+0.00986+0.00992+0.02937)}=0.30
\end{gathered}
$$




\section{APPENDIX B - INITIAL INVITATION TO EXPERTS}

Dear Subject Matter Expert,

I am a PhD candidate in the Department of Engineering and Technology Management at Portland State University. The topic of my research is technology transfer and its title is: "A Scoring Model to Assess Organizations' Technology Transfer Capabilities".

I am building a multi-criteria decision model to develop a technology transfer score to measure organizations' technology transfer capabilities. In order to properly build the model, I will use subject matter experts (SME's) judgments for validation and quantification purposes.

I am hereby inviting you to participate in this study by being one of the SME's that will help me with the model validation and quantification. Your background and expertise will be very helpful to my research.

If you accept the invitation, you will receive online survey instruments, which will be used to collect your judgments. Below is a summary of the participation regarding time commitment:

- Validation Phase: a maximum of 2 online surveys, ranging from 2 to 5 minutes each

- Quantification Phase: a maximum of 3 online surveys, ranging from 5 to 15 minutes each

- The time period between each survey will range from a few days up to several weeks, depending on how quickly other experts respond

I will be honored if you accept my invitation, and will appreciate it greatly if you also suggest other experts on technology transfer as potential expert panel members. Please notice that the Portland State University's Institutional Review Board (IRB) has approved this study. The participation in this study does not 
involve any risks of any kind whatsoever. Moreover, your name will be kept in total confidentiality and will not be used in any published reports.

Please fill out the form below and return it to me at your earliest convenience. I look forward to receiving your reply.

[ ] Yes, I accept the invitation and wish to participate in this study as an expert.

\section{Full name:}

\section{Organization:}

\section{Position:}

Please fill the fields below in case you wish to suggest other experts to participate in this study:

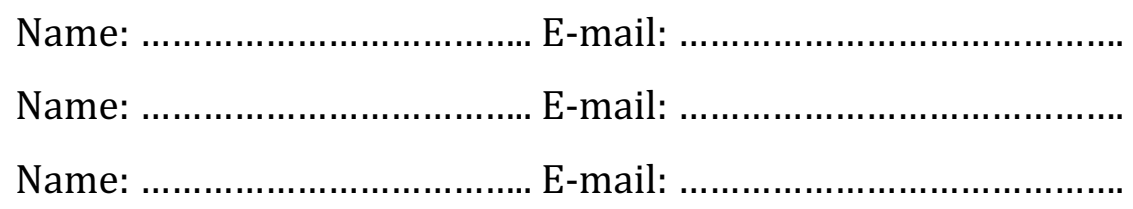

Sincerely,

Joao Ricardo Lavoie - PhD Candidate Engineering and Technology Management Department Portland State University 


\section{APPENDIX C - INVITATION FOR PERSPECTIVES VALIDATION}

Dear Expert,

Thank you for accepting to participate in my research. At this point, I ask you to help me validate the perspectives that contribute to technology transfer capabilities in an organization. The preliminary perspectives have been identified in the literature and are listed on the survey instrument that I am sending herein.

Please click on the following link to access the survey instrument. https://portlandstate.qualtrics.com/jfe/form/SV_8tUwtTcEoU2byT3

You will see the instructions to submit your response after you click on the link. I would appreciate if you fill out the survey instrument at your earliest convenience. Subsequent steps will be sent later in other emails.

Please find attached documents with further information.

If you have any questions or comments, please contact me.

Thank you,

Joao Ricardo Lavoie - PhD Student

Department of Engineering and Technology Management

Portland State University 


\section{APPENDIX C - RESEARCH INSTRUMENT 1 (RI1) - PERSPECTIVES VALIDATION SURVEY}

Welcome expert! Thank you very much for participating, I really value your input, as well as you time and attention. Next you will be prompted to validate my model's perspectives - following is a picture of the first two layers of the model

If you have any questions, please check the material send to you on the email and/or feel free to contact me at any time.

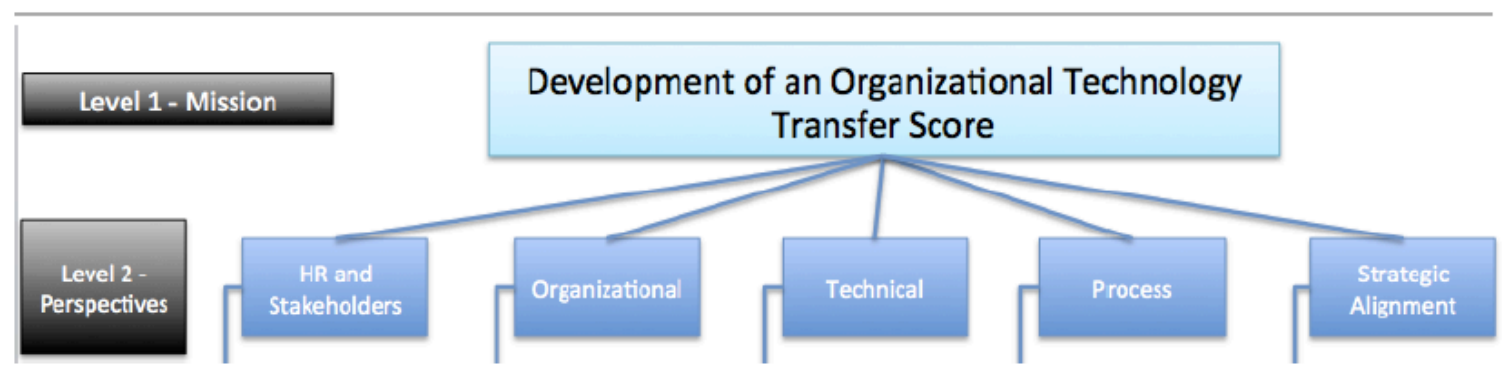

Please enter your name

The objective of the research is to develop a technology transfer score, aiming to help organizations in measuring and enhancing their internal technology transfer capabilities, ultimately leading to better R\&D performance. The methodology is Action Research followed by Hierarchical Decision Modeling (HDM).

\begin{tabular}{|c|c|}
\hline Perspective & Description \\
\hline Human Resources and Stakeholders & $\begin{array}{l}\text { Factors related to the team, training, } \\
\text { ecosystem, senior management and other } \\
\text { players involved. }\end{array}$ \\
\hline Organizational & $\begin{array}{l}\text { Factors related to organizational } \\
\text { characteristics that should be in place for a } \\
\text { better } \pi \text { process. }\end{array}$ \\
\hline Technical & $\begin{array}{l}\text { Factors related to technical assessments and } \\
\text { technology quantifications. }\end{array}$ \\
\hline Process & $\begin{array}{l}\text { Factors related to the TT process features and } \\
\text { characteristics. }\end{array}$ \\
\hline Strategic Alignment & $\begin{array}{l}\text { Factors related to the alignment between R\&D } \\
\text { and business strategy, and the integration of } \\
\text { TT with other managerial processes. }\end{array}$ \\
\hline
\end{tabular}


Based on your expertise, would you agree to include the following perspectives in the model?

\begin{tabular}{l|cc} 
& \multicolumn{2}{|c}{ Include in the model? } \\
& Yes & No \\
\hline HR and Stakeholders & $\odot$ & \\
Organizational & $\odot$ \\
Technical & $\odot$ & \\
Process & $\odot$ & \\
Strategic Alignment & $\odot$
\end{tabular}

Based on your expertise, would you add any other perspectives to the model? If yes, please name and explain the perspectives you would like to add.

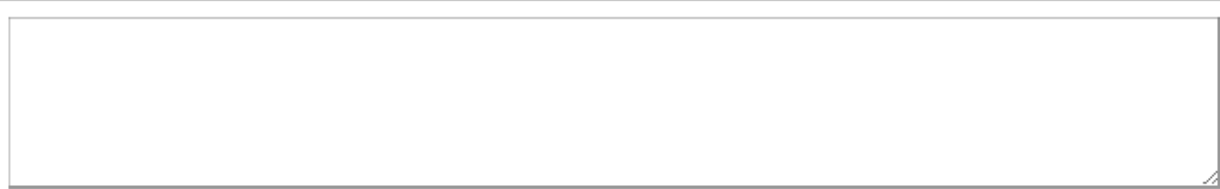




\section{APPENDIX D - INVITATION FOR FACTORS VALIDATION}

Dear Expert,

Thank you for accepting to participate in my research as a subject matter expert.

The first level of the model has been validated (the perspectives). At this point, I ask you to help me validate the factors that contribute to technology transfer capabilities in an organization, specifically the factors related to the perspective "Human Resources and Stakeholders". The preliminary factors have been identified in the literature and are listed on the survey instrument that I am sending herein.

Please click on the following link to access the survey instrument. https://portlandstate.qualtrics.com/jfe/form/SV_2ryuqDCxAo1jYYR

You will see the instructions to submit your response after you click on the link. I would appreciate if you fill out the survey instrument at your earliest convenience. Subsequent steps will be sent later in other emails.

If you have any questions or comments, please contact me.

Thank you,

Joao Ricardo Lavoie - PhD Candidate Department of Engineering and Technology Management Portland State University 


\section{APPENDIX E - RESEARCH INSTRUMENT 2 (RI2) - FACTORS VALIDATION SURVEY (SAMPLE)}

Welcome expert! Thank you very much for participating, I really value your input, as well as you time and attention.

Next you will be prompted to validate some of the factors in my model.

If you have any questions, please check the material sent to you in the email and feel free to contact me at any time.

Please enter your name

test

Please enter your sector, e.g., academia, energy, research center, construction, manufacturing, etc.

test

Please enter the name of your organization

test

Please enter your title / position, e.g., Professor, Project Manager, Director, Vice-

President, etc.

test

Please enter the country where you live (optional)

test

The objective of the research is to develop a technology transfer score, aiming to help organizations in measuring and enhancing their technology transfer capabilities, ultimately leading to better R\&D

performance. The methodology is Action Research followed by Hierarchical Decision Modeling (HDM).

Below is a picture of the Organizational factors within the model.

Below is a picture of the model. At this point you will be validating the "HR and Stakeholders" factors only, as highlighted in the figure below. The remaining factors will be validated by other expert panels. 


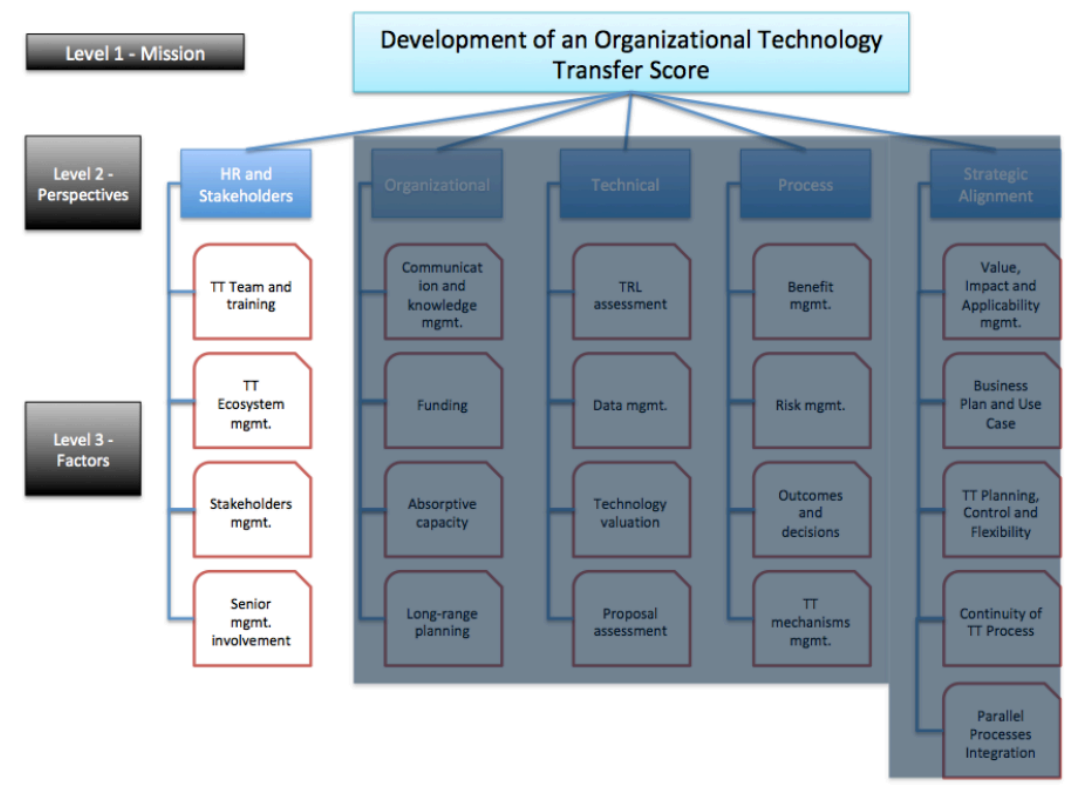

Below is a brief description of the "HR and Stakeholders" factors

\begin{tabular}{|c|c|}
\hline \multicolumn{2}{|r|}{ HR and Stakeholders } \\
\hline Factor & Description \\
\hline$\Pi \pi$ team and training & $\begin{array}{l}\text { Is there a dedicated } \Pi \text { team? Background of people in the team; is the team } \\
\text { multidisciplinary? Type and frequency of training. }\end{array}$ \\
\hline Tा Ecosystem mgmt. & $\begin{array}{l}\text { Relationship, cooperation and partnership with } \Pi \text { ecosystem members, e.g., } \\
\text { TO's, Science Parks, Research Centers, incubators, POC Centers, etc. }\end{array}$ \\
\hline Stakeholders mgmt. & $\begin{array}{c}\text { Interactions and cooperation with internal and external stakeholders to } \\
\text { increase participation and create awareness (within the boundaries of the TT } \\
\text { process). }\end{array}$ \\
\hline $\begin{array}{l}\text { Senior mgmt. } \\
\text { involvement }\end{array}$ & $\begin{array}{c}\text { Awareness, approval, support and active participation of senior managers in } \\
\text { the } \pi \text { process, including the indication of } \Pi \text { champions. }\end{array}$ \\
\hline
\end{tabular}

Based on your expertise, would you agree to include the following factors in the model under the "HR and stakeholders" perspective? 


\begin{tabular}{l|cc} 
& Include in the model? \\
\hline TT team and training & Yes & No \\
TT Ecosystem mgmt. & $\bullet$ \\
Stakeholders mgmt. & \\
Senior mgmt. involvement & -
\end{tabular}

Based on your expertise, would you add any other factors under this perspective? If yes, please name and explain the factors you would like to add. Also, if you selected "no" for any of the factors listed in the previous question, please explain your reasons. 


\section{APPENDIX F - INVITATION FOR PERSPECTIVES QUANTIFICATION}

Dear expert,

After several rounds of data collection, the model to determine the organizational technology transfer score has been validated. The perspectives and factors in the model were approved by at least $75 \%$ of the experts.

I now ask you to quantify the relative importance of each perspective in terms of its contribution to an organization's technology transfer capabilities.

The constant-sum method will be used for pairwise comparisons between perspectives to determine the importance weight of each perspective.

Please access the following link for the pairwise comparisons. (It will take approximately 3-5 minutes to complete).

\section{https://portlandstate.qualtrics.com/jfe/form/SV_6ERly0l734GPivz}

You will see the instructions to submit your response after you click on the link. I would appreciate if you fill out the survey instrument at your earliest convenience. Subsequent steps will be sent later in other emails.

Please find attached documents with further information.

If you have any questions or comments, please contact me.

Thank you,

Joao Ricardo Lavoie - PhD Student

Department of Engineering and Technology Management

Portland State University 


\section{APPENDIX G - RESEARCH INSTRUMENT 3 (RI3) - PERSPECTIVES \\ QUANTIFICATION INSTRUMENT (SAMPLE)}

Welcome expert! Thank you very much for participating, I really value your input, as well as you time and attention.

Next you will be prompted to quantify my model's perspectives - following is a picture of the validated model

If you have any questions, please check the material sent to you via email and feel free to contact me at any time.

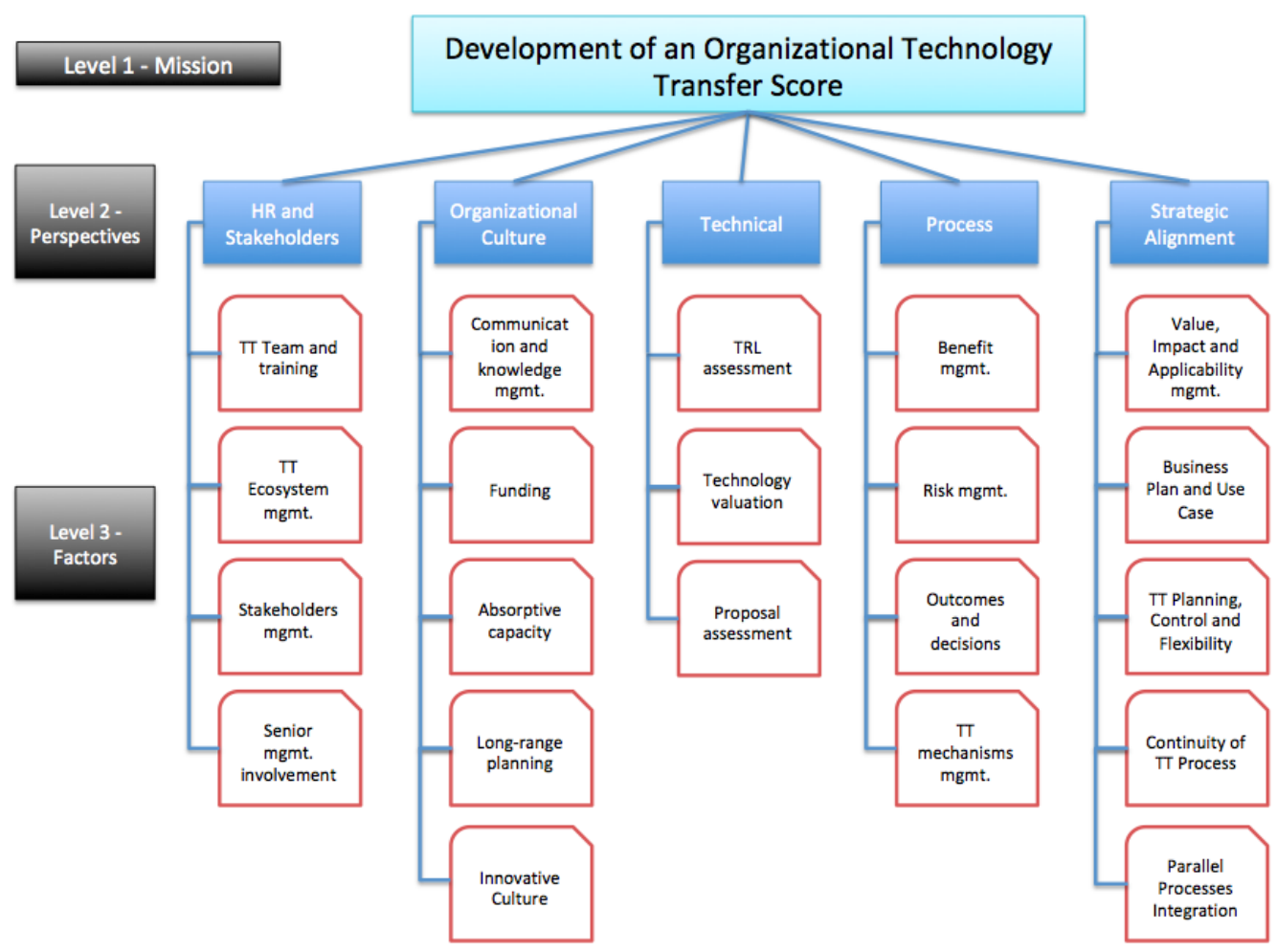


Please enter your name

test

Please enter your sector, e.g., academia, energy, research center, construction, manufacturing, etc.

test

Please enter the name of your organization

test

Please enter your title/position, e.g., Professor, Project Manager, Director, Vicepresident, etc.

test

Please enter the country where you live (optional)

test

The objective of the research is to develop a technology transfer score, aiming to help organizations in measuring and enhancing their technology transfer capabilities, ultimately leading to better R\&D performance. The methodology is Action Research followed by Hierarchical Decision Modeling (HDM).

Below is a picture of the model - the highlighted section is the one to be quantified. 


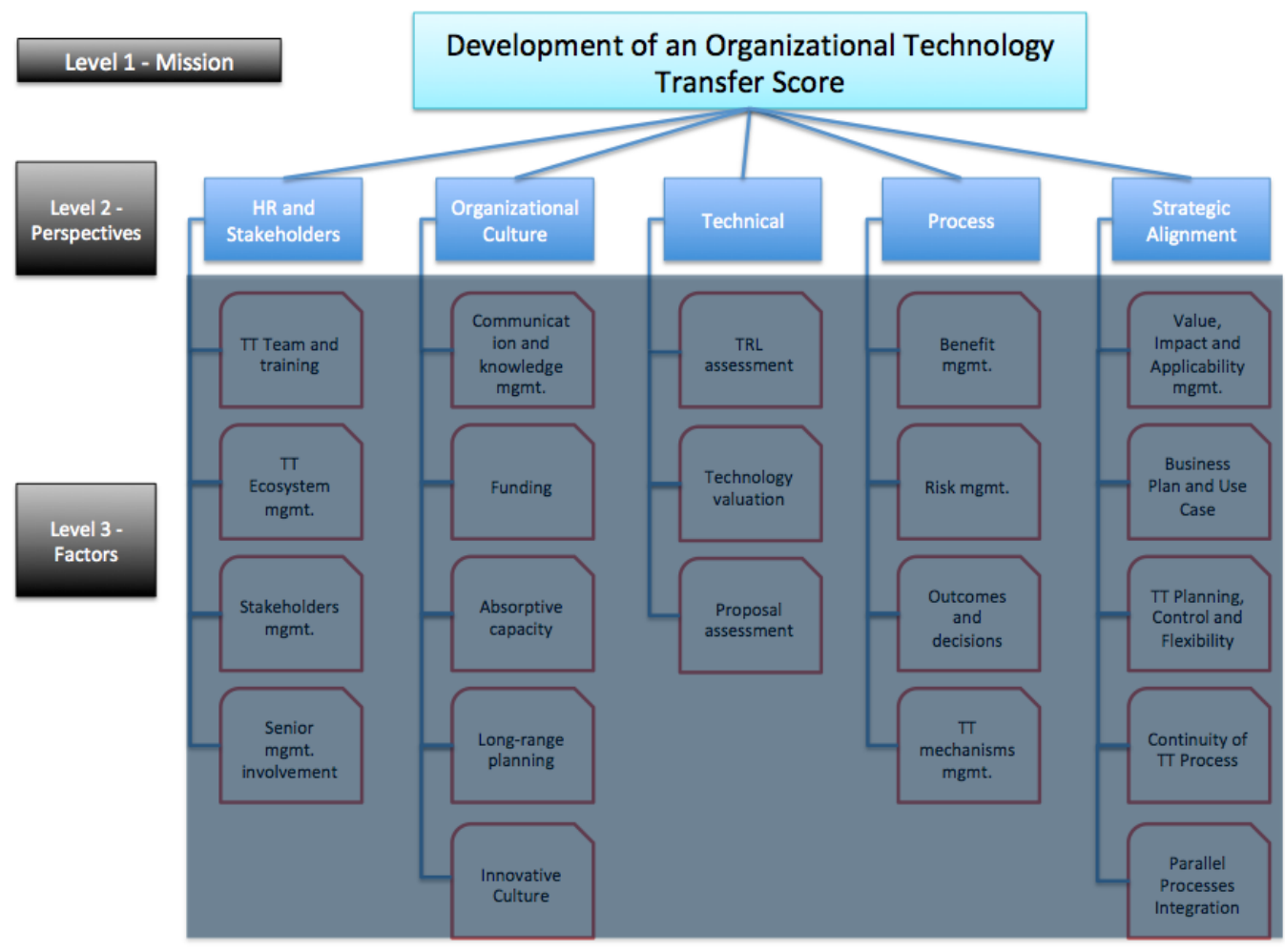

Below is a brief description of each perspective: 


\begin{tabular}{|c|c|}
\hline Perspective & Description \\
\hline Human Resources and Stakeholders & $\begin{array}{l}\text { Factors related to the team, training, } \\
\text { ecosystem, senior management and other } \\
\text { players involved. }\end{array}$ \\
\hline Organizational Culture & $\begin{array}{l}\text { Factors related to organizational } \\
\text { characteristics that should be in place for a } \\
\text { better } \pi \text { process. }\end{array}$ \\
\hline Technical & $\begin{array}{l}\text { Factors related to technical assessments and } \\
\text { technology quantifications. }\end{array}$ \\
\hline Process & $\begin{array}{l}\text { Factors related to the TT process features and } \\
\text { characteristics. }\end{array}$ \\
\hline Strategic Alignment & $\begin{array}{l}\text { Factors related to the alignment between R\&D } \\
\text { and business strategy, and the integration of } \\
\text { TT with other managerial processes. }\end{array}$ \\
\hline
\end{tabular}

You will quantify the importance of each perspective through pairwise comparisons. Please read the instructions below:

- Items will be compared against each other, in pairs. Assign the points according to your opinion.

- The assignment of points should reflect the importance of each item. Example: if $A$ is $3 x$ more important than $B$, A should receive 75 points and $B$ should receive 25 points.

- Note that for each pairwise comparison, the total of points assigned must be 100. 
- Please try to be logically consistent in your choices, i.e., if A is better than B, and $\mathrm{B}$ is better than $\mathrm{C}, \mathrm{A}$ must be better than $\mathrm{C}$.

- Furthermore, if $\mathrm{A}$ is $2 \mathrm{x}$ better than $\mathrm{B}$, and $\mathrm{B}$ is $3 \mathrm{x}$ better than $\mathrm{C}, \mathrm{A}$ must be $6 \mathrm{x}$ better than $\mathrm{C}$.

Please judge the importance of the following perspectives dividing 100 points between them. Drag the bars below assigning more points to the one you deem more important.

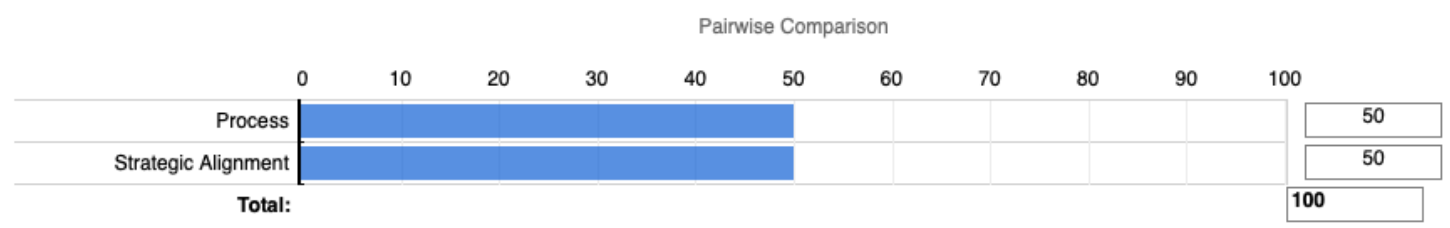




\section{APPENDIX H - INVITATION FOR FACTORS QUANTIFICATION}

Dear expert,

After several rounds of data collection, the model to determine the organizational technology transfer score has been validated. The perspectives and factors in the model were approved by at least $75 \%$ of the experts.

I now ask you to quantify the relative importance of factors under each perspective. The constant-sum method will be used for pair-wise comparisons between factors to determine the importance weight of each factor.

Please access the following link for the pairwise comparisons. (It will take approximately from 3 to 5 minutes to complete). The link below refers to the factors under the perspective "HR and Stakeholders".

\section{https://portlandstate.qualtrics.com/jfe/form/SV 2rgioLSY2VJI7bv}

You will see the instructions to submit your response after you click on the link. I would appreciate if you fill out the survey instrument at your earliest convenience. Subsequent steps will be sent later in other emails.

Please find attached documents with further information.

If you have any questions or comments, please contact me.

Thank you,

Joao Ricardo Lavoie - PhD Student

Department of Engineering and Technology Management

Portland State University 


\section{APPENDIX I - RESEARCH INSTRUMENT 4 (RI4) - FACTORS QUANTIFICATION INSTRUMENT (SAMPLE)}

Welcome expert! Thank you very much for participating, I really value your input, as well as you time and attention.

Next you will be prompted to quantify some of the model's factors, related to HR and Stakeholders - following is a picture of the validated model.

If you have any questions, please check the material sent to you via email and feel free to contact me at any time.

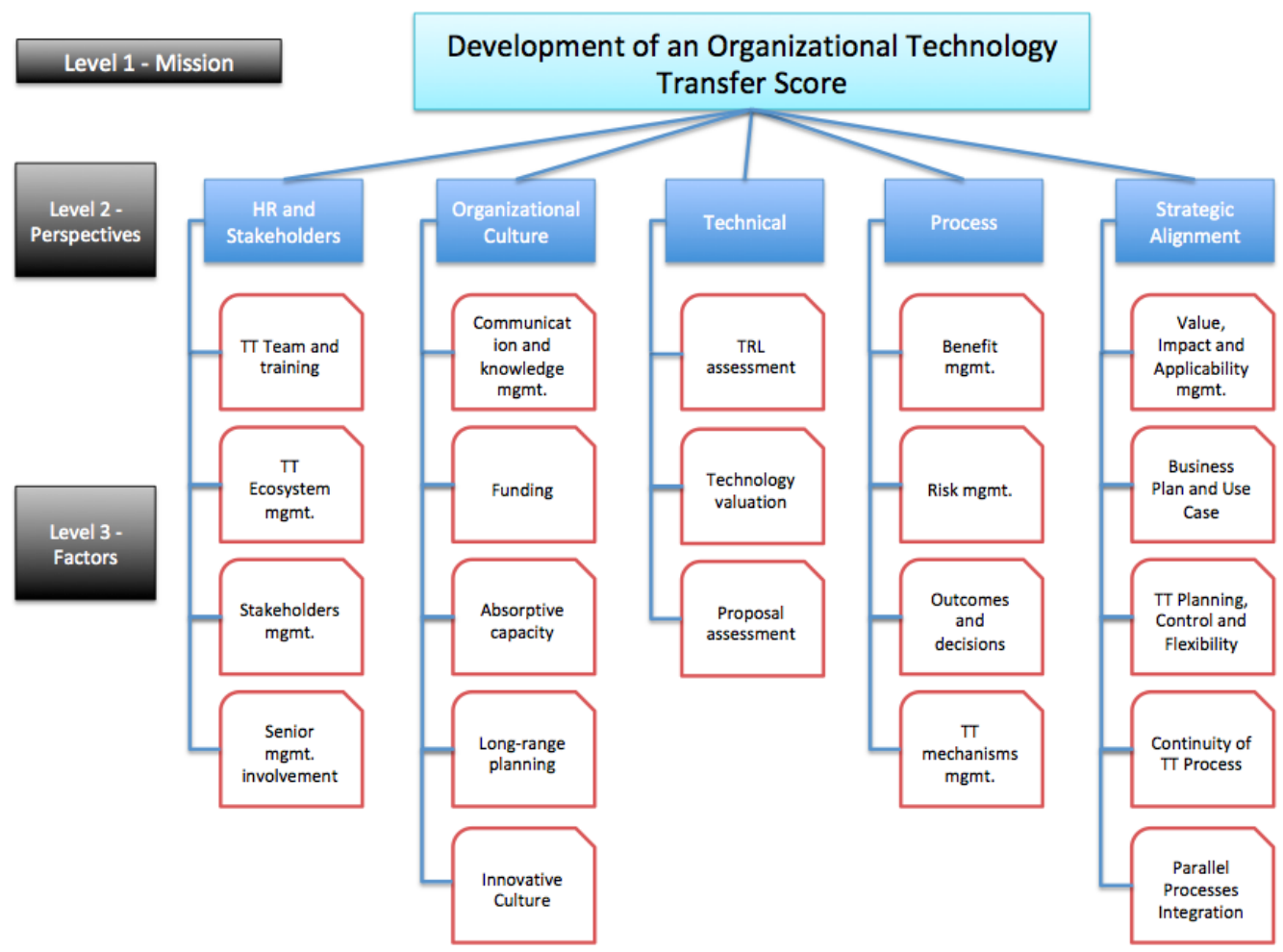

Please enter your name test 
Please enter your sector, e.g., academia, energy, research center, construction, manufacturing, etc.

test

Please enter the name of your organization

test

Please enter your title/position, e.g., Professor, Project Manager, Director, Vicepresident, etc.

test

Please enter the country where you live (optional)

test

The objective of the research is to develop a technology transfer score, aiming to help organizations in measuring and enhancing their technology transfer capabilities, ultimately leading to better R\&D performance. The methodology is Action Research followed by Hierarchical Decision Modeling (HDM).

Below is a picture of the model - the highlighted section is the one to be quantified. 


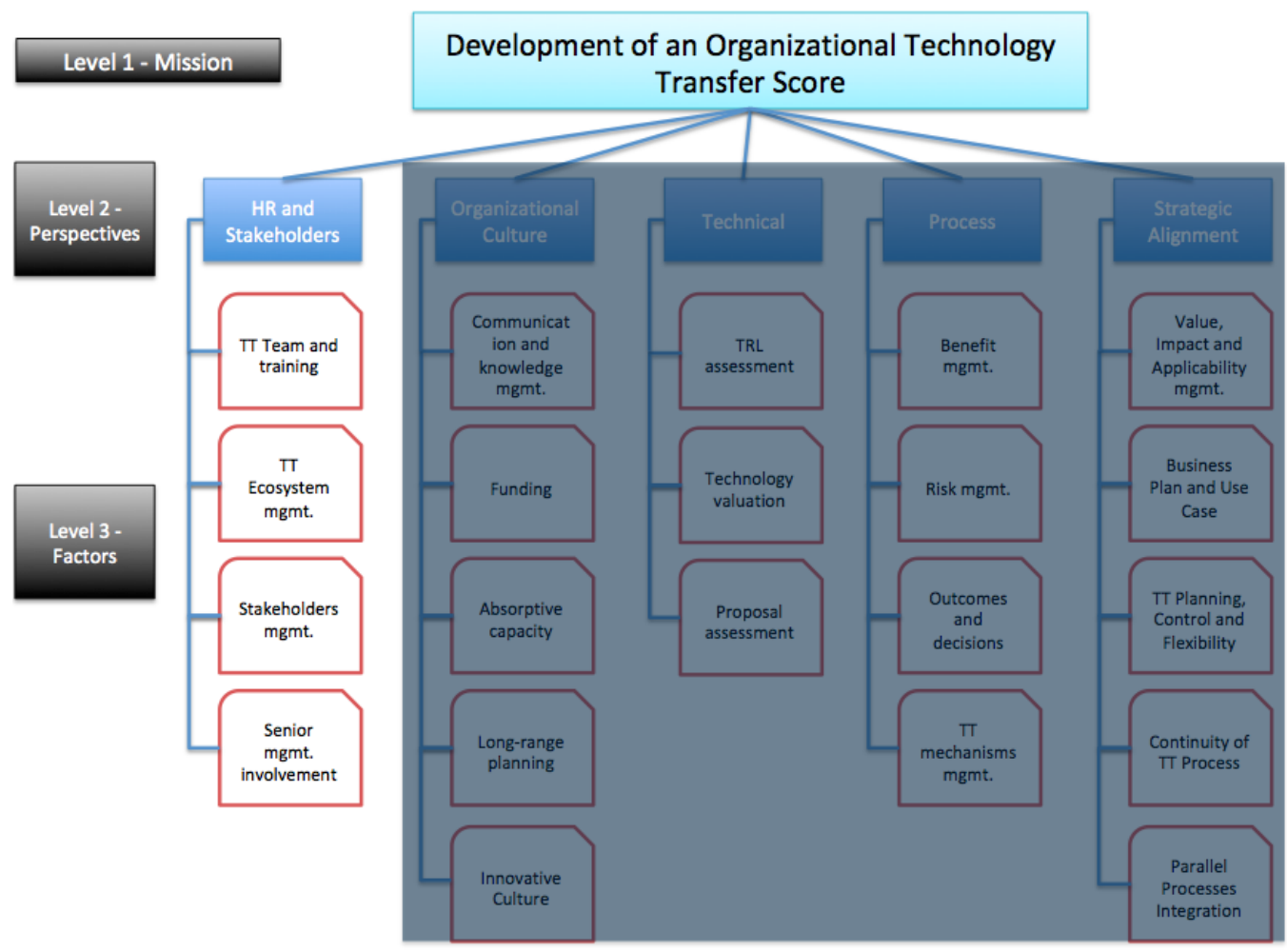

Below is a brief description of each factor:

\begin{tabular}{|c|c|}
\hline \multicolumn{2}{|r|}{ HR and Stakeholders } \\
\hline Factor & Description \\
\hline$\pi$ team and training & $\begin{array}{l}\text { Is there a dedicated } \Pi \text { team? Background of people in the team; is the team } \\
\text { multidisciplinary? Type and frequency of training. }\end{array}$ \\
\hline П Ecosystem mgmt. & $\begin{array}{l}\text { Relationship, cooperation and partnership with } \Pi \text { ecosystem members, e.g., } \\
\Pi O^{\prime} \text { 's, Science Parks, Research Centers, incubators, POC Centers, etc. }\end{array}$ \\
\hline Stakeholders mgmt. & $\begin{array}{l}\text { Interactions and cooperation with internal and external stakeholders to } \\
\text { increase participation and create awareness (within the boundaries of the } \pi T \\
\text { process). }\end{array}$ \\
\hline $\begin{array}{l}\text { Senior mgmt. } \\
\text { involvement }\end{array}$ & $\begin{array}{l}\text { Awareness, approval, support and active participation of senior managers in } \\
\text { the } \Pi \text { process, including the indication of } \Pi \text { champions. }\end{array}$ \\
\hline
\end{tabular}

You will quantify the importance of each factor through pairwise comparisons. Please read the instructions below:

- Items will be compared against each other, in pairs. Assign the points according to your opinion.

- The assignment of points should reflect the importance of each item. Example: if $A$ is $3 x$ more important than $B$, A should receive 75 points and $B$ should receive 25 points. 
- Note that for each pairwise comparison, the total of points assigned must be 100.

- Please try to be logically consistent in your choices, i.e., if $A$ is better than $B$, and $\mathrm{B}$ is better than $\mathrm{C}, \mathrm{A}$ must be better than $\mathrm{C}$.

- Furthermore, if $A$ is $2 x$ better than $B$, and $B$ is $3 x$ better than $C, A$ must be $6 x$ better than $\mathrm{C}$.

Please judge the importance of the following factors dividing 100 points between them. Drag the bars below assigning more points to the one you deem more important.

\begin{tabular}{|c|c|c|c|c|c|c|c|c|c|c|c|}
\hline \multicolumn{12}{|c|}{ Pairwise Comparison } \\
\hline & 0 & 10 & 20 & 30 & 40 & 50 & 60 & 70 & 80 & 90 & 100 \\
\hline$\pi$ Team and Traning & & & & & & & & & & & 50 \\
\hline TT Ecosystem mgmt. & & & & & & & & & & & 50 \\
\hline
\end{tabular}

